\title{
Petrophysical Characterization and Reservoir Simulator for Methane Gas Production from Gulf of Mexico Hydrates
}

\author{
Final Report
}

Reporting Period: July 2, 2002 through June 30, 2006

Prepared By: Kishore Mohanty, Bill Cook, Mustafa Hakimuddin, Dr. Ramanan Pitchumani, Dr. Damiola Ogunlana, Dr. Jon Burger, and John Shillinglaw

Issued: June 2007

Department of Energy Award No. DE-FC26-02NT41327

\author{
Intertek Westport Technology Center \\ 6700 Portwest Drive \\ Houston, Texas 77024 \\ (713) 479-8400 (Tel.) \\ (713) 864-9357 (Fax) \\ www.westport1.com \\ and \\ University of Houston \\ Department of Chemical Engineering \\ 4800 Calhoun Road \\ Houston, TX 77204-4004
}

Prepared for The U.S. Department of Energy

This report was prepared with the support of The U.S. Department of Energy, under Award No. DE-FC2602NT41327. However, any opinions, findings, conclusions, or recommendations expressed herein are those of the author and do not necessarily reflect the views of the DOE. Intertek Westport Technology Center and the University of Houston make no representations or warranties, either expressed or implied, and specifically provides the results of this manual "as is". 


\section{DISCLAIMER}

This report was prepared as an account of work sponsored by an agency of the United States Government. Neither the United States Government nor any agency thereof, nor any of their employees, makes any warranty, expressed or implied, or assumes any legal liability or responsibility for the accuracy, completeness, or usefulness of any information, apparatus, product, or process disclosed, or represents that its use would not infringe privately owned rights. Reference herein to any specific commercial product, process or service by trade name, trade mark, manufacturer, or otherwise does not necessarily constitute its endorsement, recommendation or favoring by the United States Government or any agency thereof. The views and opinions of the authors expressed herein do not necessarily reflect those of the United States Government or any agency thereof. 


\begin{abstract}
Gas hydrates are crystalline, ice-like compounds of gas and water molecules that are formed under certain thermodynamic conditions. Hydrate deposits occur naturally within ocean sediments just below the sea floor at temperatures and pressures existing below about 500 meters water depth. Gas hydrate is also stable in conjunction with the permafrost in the Arctic. Most marine gas hydrate is formed of microbially generated gas. It binds huge amounts of methane into the sediments. Estimates of the amounts of methane sequestered in gas hydrates worldwide are speculative and range from about 100,000 to $270,000,000$ trillion cubic feet (modified from Kvenvolden, 1993). Gas hydrate is one of the fossil fuel resources that is yet untapped, but may play a major role in meeting the energy challenge of this century.
\end{abstract}

In this project novel techniques were developed to form and dissociate methane hydrates in porous media, to measure acoustic properties and CT properties during hydrate dissociation in the presence of a porous medium. Hydrate depressurization experiments in cores were simulated with the use of TOUGHFX/HYDRATE simulator. Input/output software was developed to simulate variable pressure boundary condition and improve the ease of use of the simulator. A series of simulations needed to be run to mimic the variable pressure condition at the production well.

The experiments can be matched qualitatively by the hydrate simulator. The temperature of the core falls during hydrate dissociation; the temperature drop is higher if the fluid withdrawal rate is higher. The pressure and temperature gradients are small within the core. The sodium iodide concentration affects the dissociation pressure and rate. This procedure and data will be useful in designing future hydrate studies. 


\section{TABLE OF CONTENTS}

Page

DISCLAIMER

ABSTRACT

TABLE OF CONTENTS

$v-i x$

INTRODUCTION

EXECUTIVE SUMMARY 3

1.0 EXPERIMENTAL

1.1 Program Objectives and Scope

1.1.1 Task 1, Management Processes 6

1.1.2 Task 2, Develop and Produce Handbook for Transporting, Preserving, and Storing Hydrate Samples from Field to the Laboratory

1.1.3 Task 3, Characterization of Bulk System Properties of Porous Media and Gas, Water, and Hydrates

1.1.4 Tasks 4, 5 and 6, Formation and Dissociation of Hydrates in Well Characterized Consolidated Porous Media

1.1.5 Task 7 and 8, Formation and Dissociation of Hydrates in GOM Unconsolidated Sediments

1.1.6 Task 9, Geophysical Properties of Hydrate Bearing Sediment

1.1.7 Task 10 and 11, Develop Numerical Models/Correlations and Incorporate Petrophysical Models into Reservoir Simulator for Scale up $\quad 9$

1.1.8 Task 12, Produce Hydrate Core Handling and Characterization Manual $\quad 10$

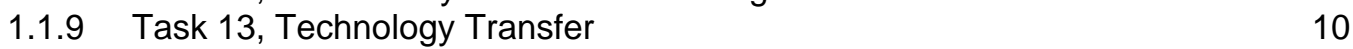

1.1.10 Task 14, Westport Cost Share Activities $\quad 10$

1.2 Experimental Apparatus Design 11

$\begin{array}{lll}1.3 & \text { Material Characterizations } & 15\end{array}$

$\begin{array}{ll}\text { 1.3.1 Synthetic Core Material Selection } & 15\end{array}$

$\begin{array}{ll}\text { 1.3.2 Core Properties } & 15\end{array}$

$\begin{array}{lll}\text { 1.3.3 Geological Characterization } & 15\end{array}$

1.3.4 Geomechanical Properties 24

$1.4 \quad$ Fluid System $\quad 29$

$\begin{array}{lll}1.5 & \text { System Test } & 30\end{array}$

2.0 NUMERICAL SIMULATION METHODS

$2.1 \quad$ Modeling Methods $\quad 41$

2.2 Simulator Input Output Processing

$\begin{array}{lll}3.0 & \text { RESULTS AND DISCUSSION } & 45\end{array}$

3.1 Experimental Results $\quad 45$

3.1.1 Initial Gas Hydrate Generation Test, No Core Material (Test 1) 46

3.1.2 Hydrate Formation in High Permeability Core (Test 2) 52

3.1.3 Hydrate Formation in High Permeability Core (Test 3) 60

3.1.4 Hydrate Formation in QF10 Medium Permeability Core (Test 4) 67

3.1.5 Hydrate Formation in QF10 Medium Permeability Core (Test 5)

3.1.6 Hydrate Formation in QF10 Medium Permeability Core (Test 6) 80

3.1.7 Hydrate Formation in QF10 Medium Permeability Core (Test 7) 86

3.1.8 Hydrate Formation in QF10 Medium Permeability Core (Test 8) 91

3.1.9 Hydrate Formation in QF10 Medium Permeability Core (Test 9) 95 
3.1.10 Hydrate Formation in QF10 Medium Permeability Core (Test 10) 99

Page

3.1.11 Hydrate Formation in QF10 Medium Permeability Core (Test 11) 107

3.1.12 Hydrate Formation in QF10 Medium Permeability Core (Test 12) 115

3.1.13 Hydrate Formation in Simulated Gulf of Mexico Sediment (Test 13) 123

3.1.14 Discussion

3.2 Modeling Results

Comparison of Model Output to Test 10

$\begin{array}{lll}\text { 3.2.2 Comparison of Model Output to Test } 11 & 142\end{array}$

$\begin{array}{lll}\text { 3.2.3 Comparison of Model Output with Test } 12 & 151\end{array}$

3.2.4 Comparison of Model Output to Test $13 \quad 166$

$\begin{array}{lll}3.3 & \text { Technology Transfer } & 175\end{array}$

$\begin{array}{lll}4.0 & \text { CONCLUSIONS } & 177\end{array}$

$\begin{array}{lr}\text { ACKNOWLEDGEMENTS } & 179\end{array}$

$\begin{array}{ll}\text { REFERENCES } & 181\end{array}$

$\begin{array}{ll}\text { APPENDICES } & 183\end{array}$

APPENDIX A - Thin Section Descriptions $\quad$ A-1

APPENDIX B - User Manual for Hydrate Simulator Pre/Post Processing B-1 


\section{LIST OF TABLES AND FIGURES}

Page

Tables

Table 1.1

Table 1.2

Table 1.3

Table 1.4

Table 1.5

Table 1.7

Table 1.8

Table 3.1.1

Table 3.1.2

Table 3.2
Summary of Routine Core Analysis Results

15

$\begin{array}{ll}\text { X-Ray Diffraction Analysis } & 17\end{array}$

$\begin{array}{lr}\text { Thin Section Description Summary } & 17\end{array}$

Typical Unconsolidated Sediment 20

Synthetic Unconsolidated Sediment 20

Summary of Unconfined Compressive Strength Tests 27

Summary of Acoustic Properties Data $\quad 28$

Hydrates Test Summary $\quad 45$

Measured Acoustic Velocity as a Function of Temperature during Hydrate

Formation in Bulk Sodium lodide (Nal Solution) 48

Key Parameters for the Simulators

\section{Figures}

Figure 1.1

Figure 1.2

Figure 1.3

Figure 1.4

Figure 1.5

Figure 1.6

Figure 1.7

Figure 1.8

Figure 1.9

Figure 1.10

Figure 1.11

Figure 1.12

Figure 1.13

Figure 1.14

Figure 1.15

Figure 1.16

Figure 1.17

Figure 1.18

Figure 2.1

Figure 2.2

Figure 3.1.1

Figure 3.1.2

Figure 3.1.3

Figure 3.1.4

Figure 3.1.5

Figure 3.1.6

Figure 3.1.7

Figure 3.1.8

Figure 3.1.9

Figure 3.1.10

Figure 3.1.11.

Figure 3.1.12.

Figure 3.1.13.

Figure 3.1.14.
Schematic of Experimental Apparatus for Hydrate Formation in Core Holder

12

Picture of Boot for Hydrate Formation in Porous Medium

Core Assembly

Experimental Setup (Front Side) for Hydrate Formation in Core

Experimental Setup (Back Side) for Hydrate Formation in Core 14

Mercury Injection Capillary Pressure Results $\quad 22$

Pore Size Distribution Results 23

Gas Hydrate Equilibrium for Methane 6-wt\% Nal Brine 29

Sodium lodide Ice Formation $\quad 30$

Base CT Scan at $40^{\circ} \mathrm{F} \quad 32$

First CT Scan at $40^{\circ} \mathrm{F}$ - One Day after Base Scan 33

Second CT Scan at $40^{\circ} \mathrm{F}$ - Two Days after Base Scan 34

Third CT Scan at $40^{\circ} \mathrm{F}$ - Three Days after Base Scan 35

Fourth CT Scan at $40^{\circ} \mathrm{F}$ - Four Days after Base Scan 36

Base Scan Subtracted from First Scan $\quad 37$

Base Scan Subtracted from Second Scan 38

Base Scan Subtracted from Third Scan 39

Base Scan Subtracted from Fourth Scan $\quad 40$

Plot of a core 43

Temperature Distribution across the Core 44

Test 1: Measured Acoustic Velocity as a Function of Temperature during

Hydrate Formation in Bulk Sodium lodide (Nal) Solution

49

Test 1: Flow data during Hydrate Formation Test with Bulk Sodium lodide

Solution

CT Scan Images before and after Hydrate Formation

Test 2: Hydrate Formation at co

Test 2: CT Images of Core after Draining 63-cc of Water from Core

Test 2: CT Image and Hydrate Formation $\quad 57$

Test 2: CT Image after Hydrate Formation 58

Test 2: Subtracted CT Image $\quad 59$

Test 3: Hydrate Formation at Constant Methane Pressure 62

Test 3: Core Resistance versus Elapsed Time 63

Test 3: CT Scan Results Before Cooling 64

Test 3: CT Scan Results After Hydrate Formation $\quad 65$

Test 3: CT Scan Results After Dissociation 66 
Figure 3.1.15. Figure 3.1.16. Figure 3.1.17. Figure 3.1.18. Figure 3.1.19. Figure 3.1.20. Figure 3.1.21. Figure 3.1.22. Figure 3.1.23. Figure 3.1.24. Figure 3.1.25. Figure 3.1.26. Figure 3.1.27. Figure 3.1.28. Figure 3.1.29. Figure 3.1.30. Figure 3.1.31. Figure 3.1.32. Figure 3.1.33. Figure 3.1.34. Figure 3.1.35. Figure 3.1.36. Figure 3.1.37. Figure 3.1.38. Figure 3.1.39. Figure 3.1.40. Figure 3.1.41. Figure 3.1.42. Figure 3.1.43. Figure 3.1.44. Figure 3.1.45. Figure 3.1.46. Figure 3.1.47. Figure 3.1.48. Figure 3.1.49. Figure 3.1.50. Figure 3.1.51 Figure 3.1.52. Figure 3.1.53. Figure 3.1.54. Figure 3.1.55 Figure 3.1.56 Figure 3.1.57 Figure 3.1.58 Figure 3.1.59. Figure 3.1.60. Figure 3.2.1 Figure 3.2.2 Figure 3.2.3 Figure 3.2.4 Figure 3.2.5 Figure 3.2.6 Figure 3.2.7
Test 4: Hydrate Formation at Constant Methane Pressure

Page

Test 4: Core Resistance versus Elapsed Time

Test 4: Acoustic Velocity versus Elapsed Time

Test 4: CT Scan Results During Hydrate Formation and Dissociation

Test 4: CT Scan Results After Hydrate Formation

Test 5: Hydrate Formation at Constant Methane Pressure

Test 5: Core Resistance versus Elapsed Time

Test 5: Acoustic Delay Time versus Elapsed Time

Test 5: CT Scan Results After Hydrate Formation

Test 6: Hydrate Formation at Constant Methane Pressure

Test 6: Core Resistance versus Elapsed Time

Test 6: Acoustic Delay Time versus Elapsed Time

Test 6: CT Scan Results after Hydrate Formation

Test 7: Hydrate Formation at Constant Methane Pressure

Test 7: Core Resistance versus Elapsed Time

Test 7: Acoustic Delay Time versus Elapsed Time

Test 7: CT Scan Results After Hydrate Formation

Test 8: Hydrate Formation at Constant Methane Pressure

Test 8: Core Resistivity versus Elapsed Time

Test 8: CT Scan Results after Hydrate Formation

Test 9: Hydrate Formation with Constant Methane Mass

Test 9: Core Resistance versus Elapsed Time

Test 9: CT Scan After Hydrate Formation

Test 10: Hydrate Formation with Constant Methane Mass

Test 10: Brine Injection, Depletion Volume and Production Density

Test 10: Saturation and Porosity Derived from CT Scans

Test 10: Electrical Resistance, Brine Injection and Depletion Volume

Test 10: Acoustic Delay Time, Brine Injection and Depletion Volume

Test 10 CT Scan Results After Hydrate Formation

Test 11: Hydrate Formation with Constant Methane Mass

Test 11: Brine Injection, Depletion Volume and Production Density

Test 11: Saturation and Porosity Derived from CT Scans

Test 11: Electrical Resistance, Brine Injection and Depletion Volume

Test 11: Acoustic Delay Time, Brine Injection and Depletion Volume

Test 11: CT Scan Results After Hydrate Formation

Test 12: Hydrate Formation with Constant Methane Mass

Test 12: Brine Injection, Depletion Volume and Production Density

Test 12: Saturation and Porosity Derived from CT Scans

Test 12: Electrical Resistance, Brine Injection and Depletion Volume

Test 12: Acoustic Delay Time, Brine Injection and Depletion Volume

Test 12: CT Scan Results After Hydrate Formation

Test 13: Hydrate Formation with Constant Methane Mass

Test 13: Brine Injection, Depletion Volume and Production Density

Test 13: Saturation and Porosity Derived from CT Scans

Test 13: Electrical Resistance, Brine Injection and Depletion Volume

Test 13: CT Scan Results After Hydrate Formation

Test 10: Pressure Stepping

Test 10: Average Pressure throughout the Core with Time

Test 10: Average Temperature throughout the core with Time

Test 10: Average Hydrate Saturation throughout the Core with Time

Test 10: Average Aqueous Saturation throughout the Core with Time

Test 10: Average Gas Saturation throughout the Core with Time

Test 10: Total Volume of Gas, Hydrate, Aqueous and Ice in the Core with Time
69

70

71

72

73

76

77

78

79

82

83

84

85

87

88

89

90

92

93

94

96

97

98

101

102

103

104

105

106

109

110

111

112

113

114

117

118

119

120

121

122

125

126

127

128

129

134

135

136

137

138

139

140 
Figure 3.2 .8 Figure 3.2.9 Figure 3.2.10 Figure 3.2.11 Figure 3.2.12 Figure 3.2.13 Figure 3.2.14 Figure 3.2.15 Figure 3.2.16 Figure 3.2.17 Figure 3.2.18 Figure 3.2.19 Figure 3.2.20 Figure 3.2.21 Figure 3.2.22 Figure 3.2.23 Figure 3.2.24 Figure 3.2.25 Figure 3.2 .26 Figure 3.2.27 Figure 3.2.28 Figure 3.2.29. Figure 3.2.30. Figure 3.2.31. Figure 3.2.32 Figure 3.2.33. Figure 3.2.34. Figure 3.2.35.
Test 10: Cumulative Volume of Methane Released over a Period of Time Test 11: Average Pressure throughout the Core with Time Test 11: Average Temperature throughout the Core with Time Test 11: Average Hydrate Saturation throughout the Core with Time Test 11: Average Aqueous Saturation throughout the Core with Time Test 11: Average Gas Saturation throughout the Core with Time Test 11: Total Volume of Gas, Hydrate, Aqueous and Ice in the Core with Time Test 11: Cumulative Volume of Methane Released over a Period of Time Test 12: Average Pressure throughout the Core with Time Test 12: Average Temperature throughout the Core with Time Test 12: Average Hydrate Saturation throughout the Core with Time Test 12: Average Aqueous Saturation throughout the Core with Time Test 12: Average Gas Saturation throughout the Core with Time Test 12: Total Volume of Gas, Hydrate, Aqueous and Ice in the Core with Time Test 12: Cumulative Volume of Methane Released over a Period of Time Test 12: Average Pressure throughout the Core with Time Test 12: Average Temperature throughout the core with Time Test 12: Average Hydrate Saturation throughout the Core with Time Test 12: Average Aqueous Saturation throughout the Core with Time Test 12: Average Gas Saturation throughout the Core with Time Test 12: Total Volume of Gas, Hydrate, Aqueous and Ice in the Core with Time Test 13: Average Pressure throughout the Core with Time Test 13: Average Temperature throughout the Core with Time Test 13: Average Hydrate Saturation throughout the Core with Time Test 13: Average Aqueous Saturation throughout the Core with Time Test 13: Average Gas Saturation throughout the Core with Time Test 13: Total Volume of Gas, Hydrate, Aqueous and Ice in the Core with Time Test 13: Cumulative Volume of Methane Released over a Period of Time
Page

141

144

145

146

147

148

149

150

153

154

155

156

157

158

159

160

161

162

163

164

165

168

169

170

171

172

173

174 


\section{INTRODUCTION}

By all counts, the world-wide energy demand is expected to go up significantly (about $65 \%$ to $172 \%$ assuming a net growth rate of $1 \%$ or $2 \%$ per year, respectively, over the current demand of about 80 million barrels in the next 50 years (Skov, 2003). Renewable and nuclear energy sources supply less than $15 \%$ of the total energy consumption today (Moniz \& Kenderline, 2002). Their role is not expected to increase much in the next 20-50 years, barring some drastic technological innovation in these fields. This increase in demand is expected to be met by fossil fuel (oil, gas \& coal). Gas hydrates may be one of the fossil fuel resources that is yet untapped, but may play a major role.

Gas hydrates are crystalline, ice-like compounds of gas and water molecules that are formed under certain thermodynamic conditions (Sloan, 1998). They are formed as a result of inclusion of small molecules (e.g., methane, ethane, CO2) inside the cavities of ice-like structures made by the water molecules. The hydrate formation can be represented by the reaction

$$
\mathrm{CH}_{4} \cdot \mathrm{n}_{\mathrm{H}} \mathrm{H}_{2} \mathrm{O} \Leftrightarrow \mathrm{CH}_{4}+n_{\mathrm{H}} \mathrm{H}_{2} \mathrm{O},
$$

where methane is a possible molecule in hydrate formation and (commonly known as hydrate number) is the number of water molecules required per gas molecule to form a gas hydrate. The hydrate number for methane is approximately 6 (Sloan, 1998). The methane concentration in hydrates is much higher than the solubility of methane in liquid water. In fact, the amount of methane in a unit volume of hydrate (at stable pressure and temperature) is approximately 170 times the amount in the same volume at standard conditions.

Hydrate deposits occur naturally within ocean sediments just below the sea floor at temperatures and pressures existing below about 500 meters water depth. Gas hydrate also is stable in conjunction with permafrost in the Arctic. Most marine gas hydrate is formed of microbially generated gas. It binds huge amounts of methane into the sediments. Estimates of the amounts of methane sequestered in gas hydrates worldwide are speculative and range from about 100,000 to $270,000,000$ trillion cubic feet (modified from Kvenvolden, 1993). This represents an amount of organic carbon that is twice as much as in all other fossil fuels on earth (conventional natural gas, oil, and coal) and an amount of methane that is about 3,000 times as much as what exists in the atmosphere. Gas hydrate is important primarily because it contains huge amounts of methane in a concentrated form and because it influences the physical properties of sedimentary deposits, particularly sediment strength.

Finding an efficient, safe method of producing natural gas from hydrate deposits is important commercially. The three methods being explored for hydrate production are: (1) depressurization, in which pressure is lowered inside the well encouraging the methane hydrate to dissociate, (2) thermal stimulation, in which the reservoir is heated above the dissociation temperature using hot water or steam, and (3) inhibitor injection, in which methanol or brine is used to disequilibrate the hydrate. Combinations 
of these methods can also be used for enhanced recovery of natural gas.

1-D models for formation of methane hydrate in marine sediments have been constructed by $\mathrm{Xu}$ and Ruppel and Rempel and Buffett and geological conditions have been proposed for hydrate accumulation based on the simulation results. Several models were proposed for gas production from hydrates in the past few years (Tsypkin, 1991; Ji et al., 2001; Moridis, 2002). In most analytical models, the mobility of the water phase is ignored (Ji et al., 2001). The analytical model by Tsypkin (1991) takes into account the flow of water, but it assumes a fixed relative permeability for each phase. Most of the analytical models assume equilibrium decomposition (Ji et al., 2001; Tsypkin, 1991). In these models, hydrate is assumed to dissociate instantaneously once the equilibrium condition is reached. Experimental work by Makogon (2001) and Stern (1996) suggests otherwise. Kinetic models of pure hydrate dissociation have been proposed by Kim et al. (1987) and Clarke and Bishnoi (2000, 2001). Yousif et al. (1991) have considered kinetics and multiphase flow for the depressurization process, but under isothermal conditions. Moridis (2002) has developed a module called EOSHYDR2 for a general-purpose simulator TOUGH2 that can model hydrate depressurization. It has been used to simulate the gas production from several hydrate zones at the Mallik site.

Many experimental studies have been conducted on hydrate thermodynamics and kinetics outside porous media (Sloan, 1998; Clark \& Bishnoi, 2000). However, there are only a few experimental studies of hydrates inside porous media. Yousif et al. (1991) had studied hydrate formation and dissociation in one Berea core; hydrate was formed from methane and water. Stern et al. (1996) have formed hydrate from ice and methane. Nimblett \& Ruppel (2003) have studied permeability evolution in marine sediments during hydrate formation. Waite et al. (2002) and Yun et al. (2005) have studied conductivity and compressional/shear wave velocities of sediments with gas hydrates.

The goal of this program was to develop and apply novel techniques to gather acoustic properties, kinetics and thermodynamic properties of hydrate dissociation in the presence of a porous medium. Physical and chemical properties of methane hydrates, along with the phase behavior, were studied by experiments and theoretical modeling. These data will be of immediate application in process engineering models for hydrate formation and dissociation. The data also provides essential results to develop and test models of hydrate formation and dissociation in deep sea drilling operations and can be used for the remediation and prevention of gas hydrates during drilling operations. 


\section{EXECUTIVE SUMMARY}

Gas hydrates are crystalline, ice-like compounds of gas and water molecules that are formed under certain thermodynamic conditions. Hydrate deposits occur naturally within ocean sediments just below the sea floor at temperatures and pressures existing below about 500 meters water depth. Gas hydrate is also stable in conjunction with the permafrost in the Arctic. Most marine gas hydrate is formed of microbially generated gas. It binds huge amounts of methane into the sediments. Estimates of the amounts of methane sequestered in gas hydrates worldwide are speculative and range from about 100,000 to $270,000,000$ trillion cubic feet (modified from Kvenvolden, 1993). Gas hydrate is one of the fossil fuel resources that is yet untapped, but may play a major role in meeting the energy challenge of this century.

A best practices manual on coring, core sample handling and analysis was prepared early in the project to provide immediate support to other DOE hydrates contractors. To expedite the publication, a hydrates workshop was held in October 2002 with presentations by subject matter experts. The manual was prepared and distributed in draft form in 2003. The final version was issued in August 2006.

Novel continuous flow techniques were developed to form and dissociate methane hydrates in porous media and to measure electrical, acoustic and CT properties in the presence of a porous medium. Two approaches were used: constant methane pressure and constant methane mass. The primary differences in the results were that electrical resistance increased with hydrate formation under constant methane pressure as hydrate saturation was maximized, but electrical resistance decreased with hydrate formation with constant methane mass, indicating that brine saturation increased. Hydrate depressurization experiments in cores were simulated with the use of TOUGH-FX/HYDRATE simulator. Input/output software was developed to simulate variable pressure boundary condition and improve the ease of use of the simulator. A series of simulations needed to be run to mimic the variable pressure condition at the production well.

The experiments can be matched qualitatively by the hydrate simulator. The temperature of the core falls during hydrate dissociation and the temperature drop is higher if the fluid withdrawal rate is higher. The pressure and temperature gradients are small within the core. The sodium iodide concentration affects the dissociation pressure and rate. This procedure and data will be useful in designing future hydrate studies. 


\subsection{EXPERIMENTAL}

\subsection{Program Objectives and Scope}

The objectives of this program were to develop new laboratory methodologies, services, and software tools for operators, enabling and/or improving cost effectiveness, safety, and overall resource recovery in (a) the drilling and producing of traditional gas and oil reservoirs in the presence of natural gas hydrates, or in conditions favorable to hydrate formation, and ultimately (b) the production of the natural hydrate resources. The program objectives were achieved through three project objectives.

1. To quantify the effects of various properties of hydrates and sediments on petrophysical properties, new and well designed laboratory experiments were performed on cores monitored with multiple sensors. Techniques and "best practices" for handling and testing cores obtained from gas hydrate bearing reservoirs were provided. A new suite of laboratory services and laboratory "best practices" were created towards the generation of required parametric knowledge to feed software tools for specific reservoir appraisal, well construction planning and life-of-field management exercises.

2. A comprehensive data set was developed on petrophysical (electrical, acoustic, etc.) and reservoir engineering properties of hydrate bearing or favorable formations for plugging into existing wellbore stability models. Using the new laboratory procedures for characterization defined above, the effects of varied sediment content on the petrophysical and reservoir engineering properties of hydrate cores were determined.

3. An initial project objective was to develop a comprehensive reservoir simulation model to predict the formation and disassociation of hydrates and subsequent flow capabilities of gas and water phases in sediments by developing a new reservoir model and hydrates simulator. This objective was later modified to adapt the Lawrence Berkeley National Laboratory "TOUGH2" reservoir model and hydrates module (later named "TOUGHFx" and "TOUGH+") for this purpose.

The scope of work was broken out into fourteen work breakdown structure activities, or "tasks:"

Task 1 Management Processes

Task 2 Develop and Produce Handbook for Transporting, Preserving, and Storing Hydrate Samples from Field to the Laboratory

Task 3 Characterization of Bulk System Properties of Porous Media and Gas, Water, and Hydrates

Task 4 Formation of Hydrates in Well Characterized Consolidated Porous Media: 
Task 5 Dissociation of Hydrates in Well Characterized Consolidated Porous Media:

Task $6 \quad$ Repeat of Task 4 and 5

Task $7 \quad$ Formation of Hydrates in GOM Unconsolidated Sediments

Task 8 Dissociation of Hydrates in GOM Unconsolidated Sediments

Task 9 Determine Geophysical Properties of Hydrate Bearing Sediments

Task 10 Develop Numerical Models/Correlations

Task 11 Incorporate Petrophysical Models into Reservoir Simulator for Scale up

Task 12 Produce Hydrate Core Handling and Characterization Manual

Task 13 Technology Transfer

Task 14 Westport Cost Share Activities

\subsubsection{Task 1, Management Processes}

This task included subtasks to prepare a research management plan and technology status assessment report, organize and convene an industry lead advisory board, and collaborate with other projects in the DOE Hydrates Program. The Research Management Plan for the experimental program was completed in August 2002. A part of the Plan was a detailed schedule of the tasks to determine the estimated project completion date. The proposal schedule assumed that some tasks could be conducted concurrently, and the program would be completed in April 2005. However, when the equipment constraints were factored into the detailed schedule, the estimated completion date was October 2005.

The Advisory Board was convened in August 2002 to review the proposed Research Management Plan. Board members attending included: Dr. John Rogers, National Energy Technology Laboratory, US Department of Energy; Dr. Emrys Jones, ChevronTexaco JIP; Prof. Turgay Ertekin, Petroleum and Natural Gas Engineering, Pennsylvania State University; and Dr. Vik Rao, Technology Ventures, Halliburton Energy Services Group. The technical program was presented and approved. A best practices manual on hydrate core sample coring, handling and analysis, originally proposed to be produced at the end of the program, had been moved to the start of the program by the Department of Energy (DOE) to provide immediate support to other DOE hydrates contractors. To expedite the preparation of the manual, the Board recommended that a hydrates workshop be conducted to gather available information. Other than this change, the Research Management Plan and detailed schedule were approved.

Initial collaboration with other DOE hydrates projects began with the Maurer/Anadarko Team in preparation for drilling the Hot Ice No.1 Well in Alaska. A hydrates technology workshop was conducted at the Anadarko headquarters in October 2002. Presenters included: Bill Winters (U.SGS); Stephen Kirby (USGS), Chandra Rai (Oklahoma University), Tom Williams (Maurer Technology), Richard Sigal 
(Anadarko), Jonathan Kwan (Anadarko), Bill Lidell (Anadarko), Pierre Collee (Corpro Group) and Guy McCardle (PTS Labs).

In 2003 through 2005, contacts were made with the BP Alaska Hydrates Project Manager and members of the Chevron GOM Gas Hydrates JIP. Topics included opportunities for cooperation and the acquisition of hydrate bearing core samples for the Westport study.

\subsubsection{Task 2, Develop and Produce Handbook for Transporting, Preserving, and Storing Hydrate} Samples from Field to the Laboratory

Using the information obtained from the hydrates technology workshop conducted at Anadarko's headquarters described in 1.1.1, above, the core sampling, handling and analysis manual was drafted, edited and revised. Version 2 of the manual was circulated for industry comment in May 2003. Suggestions from the review were incorporated in the manual, and version 3 of the manual was issued in July 2003. The final version was issued in August 2006 to complete Task 12.

\subsubsection{Task 3, Characterization of Bulk System Properties of Porous Media and Gas, Water, and} Hydrates

The Statement of Project Objectives specified that three (3) different synthetic porous media with welldefined pore structures and three (3) unconsolidated GOM cores be used in the experimental work. During from the Advisory Board Meeting in August 2002, it was recommended that Westport and Chevron coordinate on the selection of core samples for Westport's experimental work so that the results complemented the Chevron GOM Gas Hydrates JIP efforts. The Advisory Board concurred. In 2003, Westport requested representative core samples from the Chevron Hydrates JIP drilling campaign, initially scheduled for the summer of 2003 and later rescheduled for 2004 . The drilling campaign was ultimately delayed until June 2005, but the request for GOM samples was ultimately declined in October 2005 due to lack of adequate number of samples. As a result, other materials had to be used to simulate unconsolidated GOM core in the experimental work.

For uniformity of data, the characterization and the experiments were performed using the same hydrates cell. The characterization studies of the synthetic porous media and gas, water, and hydrates were performed. The results are shown in Section 1.3.

\subsubsection{Tasks 4, 5 and 6, Formation and Dissociation of Hydrates in Well Characterized Consolidated Porous Media}

In actual practice, Tasks 4, 5 and 6 were combined into a single continuous task. The intent was to run three complete experiments on each core. The information sought from the experiments was the 
temperature of hydrate formation at the experimental pressure, kinetics of formation, temperature of hydrate dissociation, and kinetics of dissociation. The data collected for each experiment included:

- multiple temperatures and pressures measurements along the length of the core

- volume of gas and water/brine

- Sh, hydrate saturation, dimensionless

- Sw, water saturation, dimensionless

- $\mathrm{Sg}$, gas saturation, dimensionless

- $\mathrm{Vp}$, compressional acoustic velocity through the core

- Vs, shear acoustic velocity through the core

- $\quad \mathrm{Vp} / \mathrm{Vs}$, ratio of the compressional and shear wave velocities, representative of the Poisson's ratio

- Resistivity, ohms, along the length of the core

- $\mathrm{CT}$ images, to show distribution of hydrate saturation along the length of the core

Pressures at various points indicated pressure gradients within the system. Temperatures at various points indicated temperature gradients within the system. The volume of fluids injected/produced showed fluid injection and production for the mass balance over the course of the experiments. Production density indicated the existence of gas and water in production tubing. Electrical resistance indicated brine saturation between the probes. Acoustic velocity indicated the saturation of gas. The CT scans provided the visual imaging to indicate the water, gas and hydrate saturation distribution within cores and confirmed the distribution of hydrate formation throughout the length of the core. A chart at the bottom of each scan relate the colors in the images to the CT number. Higher CT numbers indicate greater absorbance and higher densities, and lower numbers indicate less absorbance and lower densities.

Data was continuously gathered during the hydrate phase transformation. The core was then kept at the same conditions for an additional 2-3 days to observe further changes. Then, dissociation was initiated and monitored.

\subsubsection{Task 7 and 8, Formation and Dissociation of Hydrates in GOM Unconsolidated Sediments}

The modeling of kinetics of gas hydrate formation and dissociation in porous media for Task 8 was performed. Since unconsolidated GOM hydrate bearing core samples were not available for this program, a synthetic unconsolidated core sample with low permeability closely approximating the physical properties of GOM sediments was prepared for use. It was substituted for the Berea Sandstone in the remaining tests. A description of the simulated unconsolidated core sample is provided in Section 1.3.5. Test 13 was the first of three proposed runs using the synthetic unconsolidated core material. 
A Progress Review Meeting was conducted in October 2005. During the meeting, NETL directed that the work be stopped until a decision could be made on whether to complete the work or terminate the project. Work remaining at the time was repetitions of Test 13.

In January 2006, Westport was advised by NETL that the project would be terminated for convenience. In late February, however, Westport was advised that the decision to terminate had been reversed and that the project should be completed. By that time, all of the equipment and resources were actively engaged in other projects. The cost to acquire and reassemble the equipment and continue the experimental program at that time exceeded the amount remaining in the budget.

The results from all of the tests were reviewed by Westport and the University of Houston. The data, including the results from Test 13, were determined to be valid. It was concluded that the experimental work had essentially achieved the objectives. The results from all of the tests are shown in Section 3 , Results and Discussion.

\subsubsection{Task 9, Geophysical Properties of Hydrate Bearing Sediments}

The purpose of this task was to measure the resistivity, dielectric constant, and acoustic properties of hydrate bearing sediments and develop a logging model. The intent of this task was to perform the work on actual GOM hydrate bearing core samples. Since the GOM samples could not be obtained, this task could not be completed.

\subsubsection{Task 10 and 11, Develop Numerical Models/Correlations and Incorporate Petrophysical Models into Reservoir Simulator for Scale up}

One of the primary objectives of this program was to develop numerical tools for the prediction of the hydrate formation and dissociation in porous media. The approach was to develop a new hydrates reservoir simulation model and validate it using CT visualized tests. The next step was combine the reservoir simulation model, acoustic model, and wellbore stability correlations to develop a comprehensive hydrate reservoir simulation model.

At the first Project Review Meeting held in March 2003, Westport was directed by NETL to cease development of an independent model and that the TOUGH2 simulator and hydrates module developed by the Lawrence Berkeley National Laboratory (LBNL) be used. At the time, development of the 1-D model was more than $60 \%$ complete. The numerical model was valid for finite size systems, e.g., laboratory cores. In this model, the material balance and heat balance equations could be solved for a one-dimensional finite domain. A Heaviside-type of function was used to interpolate the transport properties at the dissociation front. The pressure at the injection well could be specified, and a no flux condition could be applied at the other boundary. 
The TOUGH2 software was received in July 2003, but not the hydrates model. Some work on an interface to TOUGH2 was performed, but in the absence of the hydrates module, work on the model was suspended in August 2003. The updated TOUGHFx software that replaced TOUGH2 software and hydrates module was received in July 2005. Work was then completed on software development. The results are described in Section 3.2

\subsubsection{Task 12, Produce Hydrate Core Handling and Characterization Manual}

This was a follow-on task to Task 2. The final version of Hydrate Core Handling and Characterization Manual was issued in August 2006.

\subsubsection{Task 13, Technology Transfer}

This task included preparation of this Final Report, marketing and commercial introduction of the software, and industry education and training. Included in Section 3.3 is a list of publications and presentations that are related to the work performed. Commercial use of the software may be limited due to the requirement that industrial users must purchase a license from LBNL for the TOUGHFx (now TOUGH+) software However, colleges, universities and not for profit institutions may obtain an LBNL license at no charge.

\subsubsection{Task 14, Westport Cost Share Activities}

This was an unscheduled task for accounting purposes to capture labor, equipment and other costs that were included in Westport's cost share. 


\subsection{EXPERIMENTAL APPARATUS DESIGN}

A custom experimental apparatus was designed and fabricated to accommodate the gas hydrate experimental procedures. The apparatus consisted of an aluminum coreholder to accommodate a 10-12 inch long core and CT scanning, a PVC jacket on the outside of the coreholder to allow for temperature control, a Julabo re-circulating bath temperature controller, a Beckman 100 HPLC pump for overburden pressure control, Heise Dual PM1 Pressure transducers for pressure measurement, piston accumulators for fluid transfer (gas or liquid), a Temco back pressure regulator to maintain pore pressure, and an Isco 100D High Pressure Syringe pump for injection of fluids. The core holder was mounted on the bed of a modified Picker PQ5000 CT Scanner. A data acquisition system was also set up to collect the data. Labview was used for the automated data acquisition and archiving.

Figure 1.1, following, shows a schematic of the apparatus. The apparatus is designed to accommodate continuous counter-flow operations, i.e., brine is introduced in the top right end of the core holder and collected from the left end, and methane gas is charged to bottom left end of the core holder and collected from the right end. Figure 1.2 shows a photograph of the confining sleeve. Figure 1.3 shows a delta scan view showing the internal components and sensors. Figures 1.4 and 1.5 are photographs of the apparatus.

Various sensors were formed into the sleeve material. Sensors for temperature, ports for pressure, and ports for resistance were included in the sleeve construction. Additionally, the end-stems were modified to include acoustic signal generation and reception.

Along the length of the sleeve were ports for:

- 3 temperature sensors (for thermistor)

- 3 pressure sensors

- 4 impedance sensors

The equipment was continuously upgraded and improved during the conduct of the experimental program. Superior boot materials were evaluated and implemented, the standard large profile metal sensors were replaced with miniature sensors, and the metal grommets in the boot were replaced with non-metallic grommets. 
Figure 1.1. Schematic of Experimental Apparatus for Hydrate Formation in Core Holder

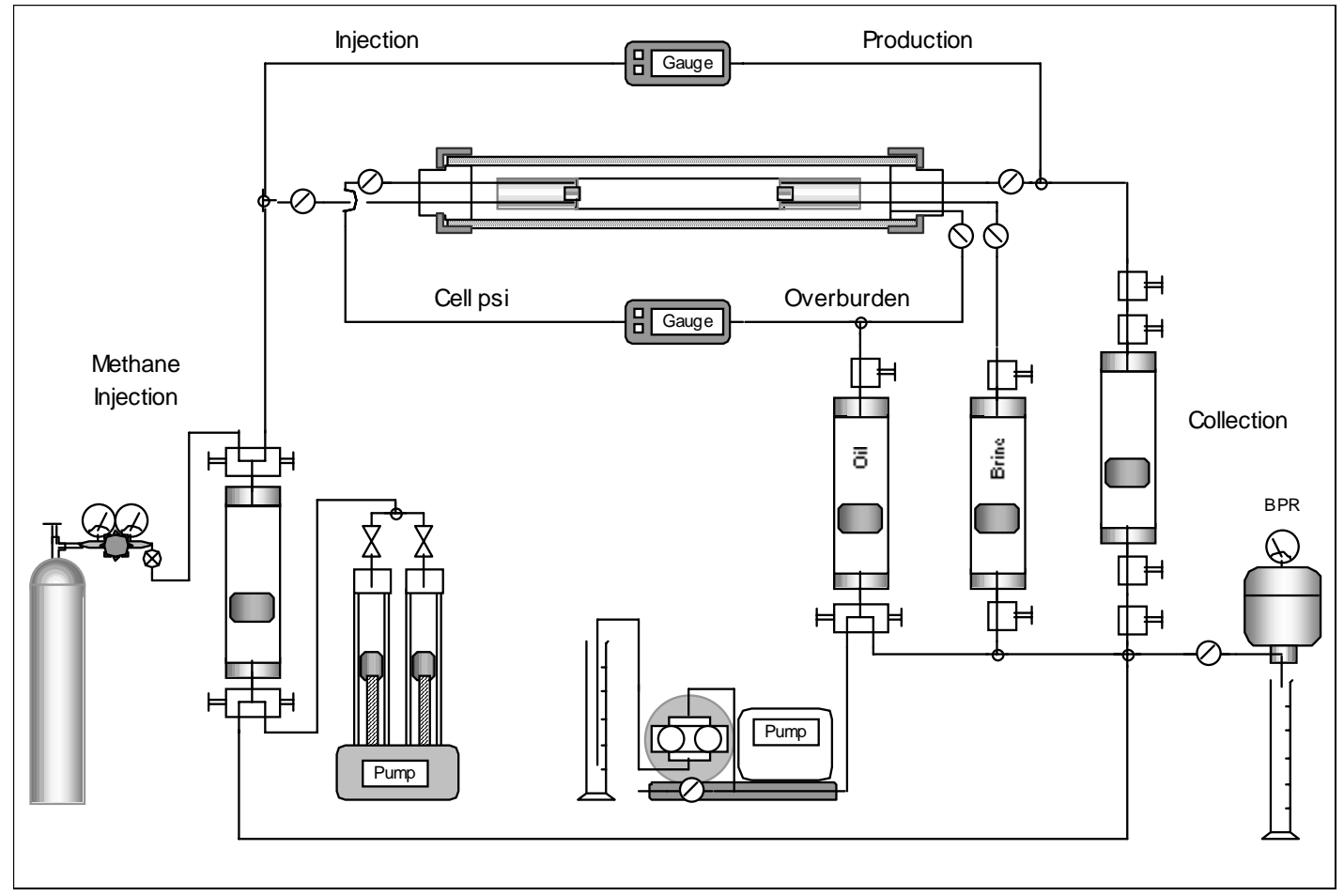

Figure 1.2. Picture of Boot for Hydrate Formation in Porous Medium

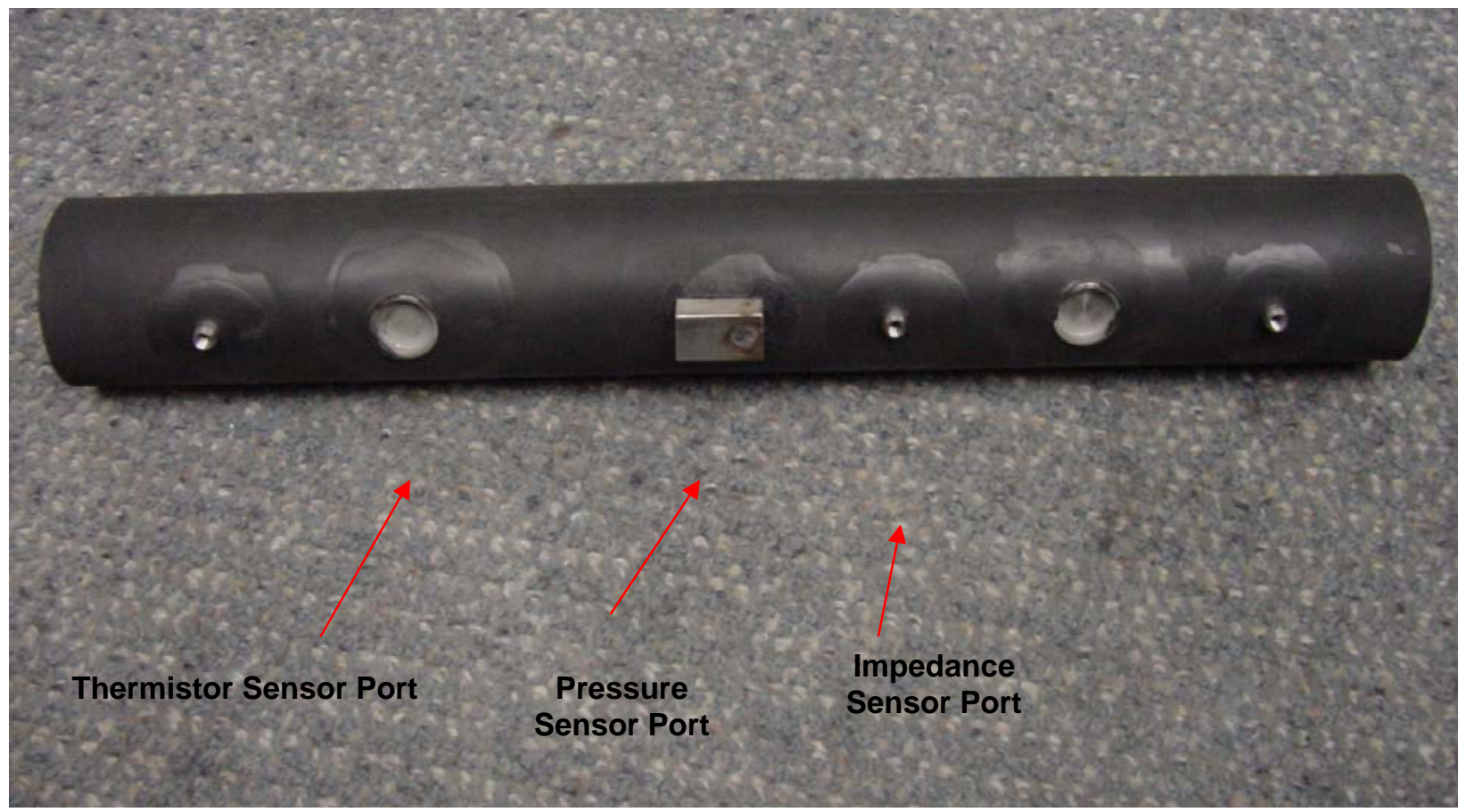


Figure 1.3. Core Assembly

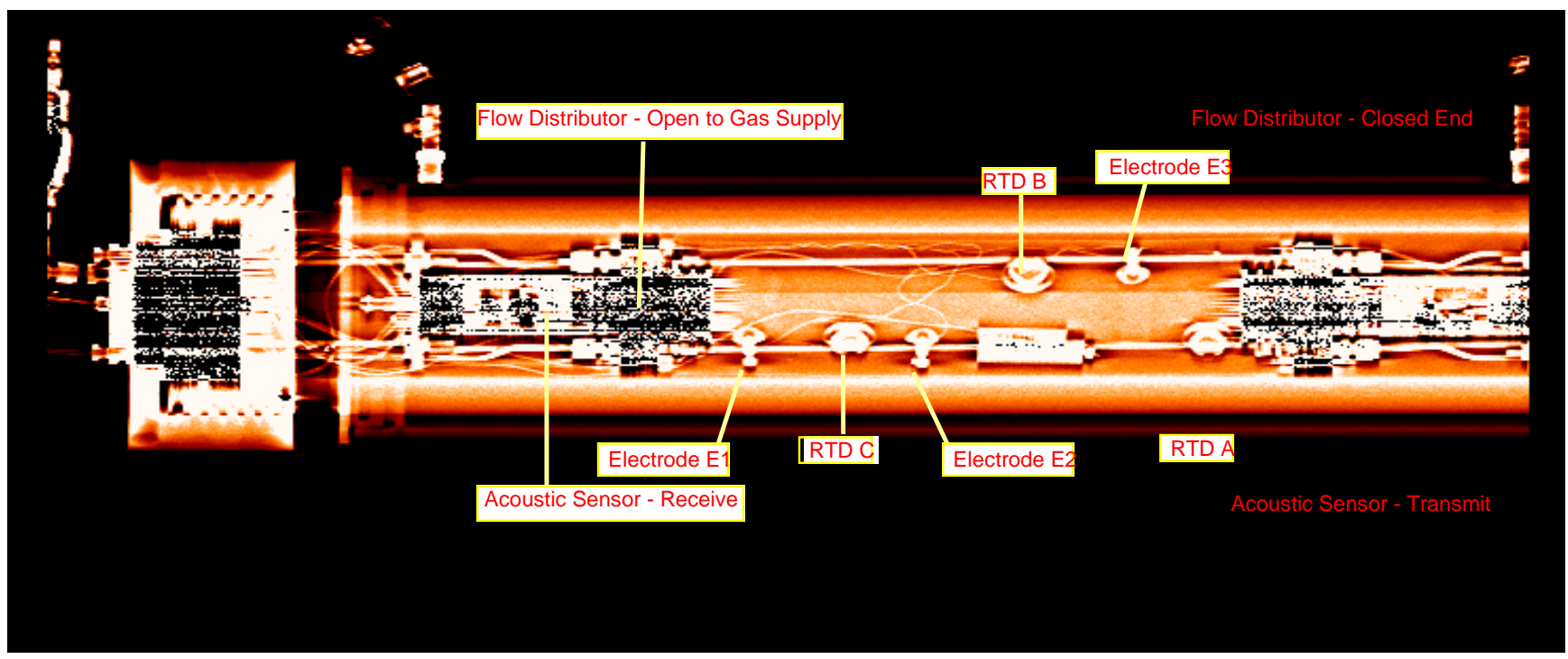

This delta scan view of the core assembly shows the locations of the electrodes for the resistance measurements and the RTDs for the temperature measurements. These components cause shadows in the individual slices along the length of the core. Slice 2 is located at the interface between the core and the flow distributor which was open to the gas supply (left side of the figure). 
Figure 1.4. Experimental Setup (Front Side) for Hydrate Formation in Core

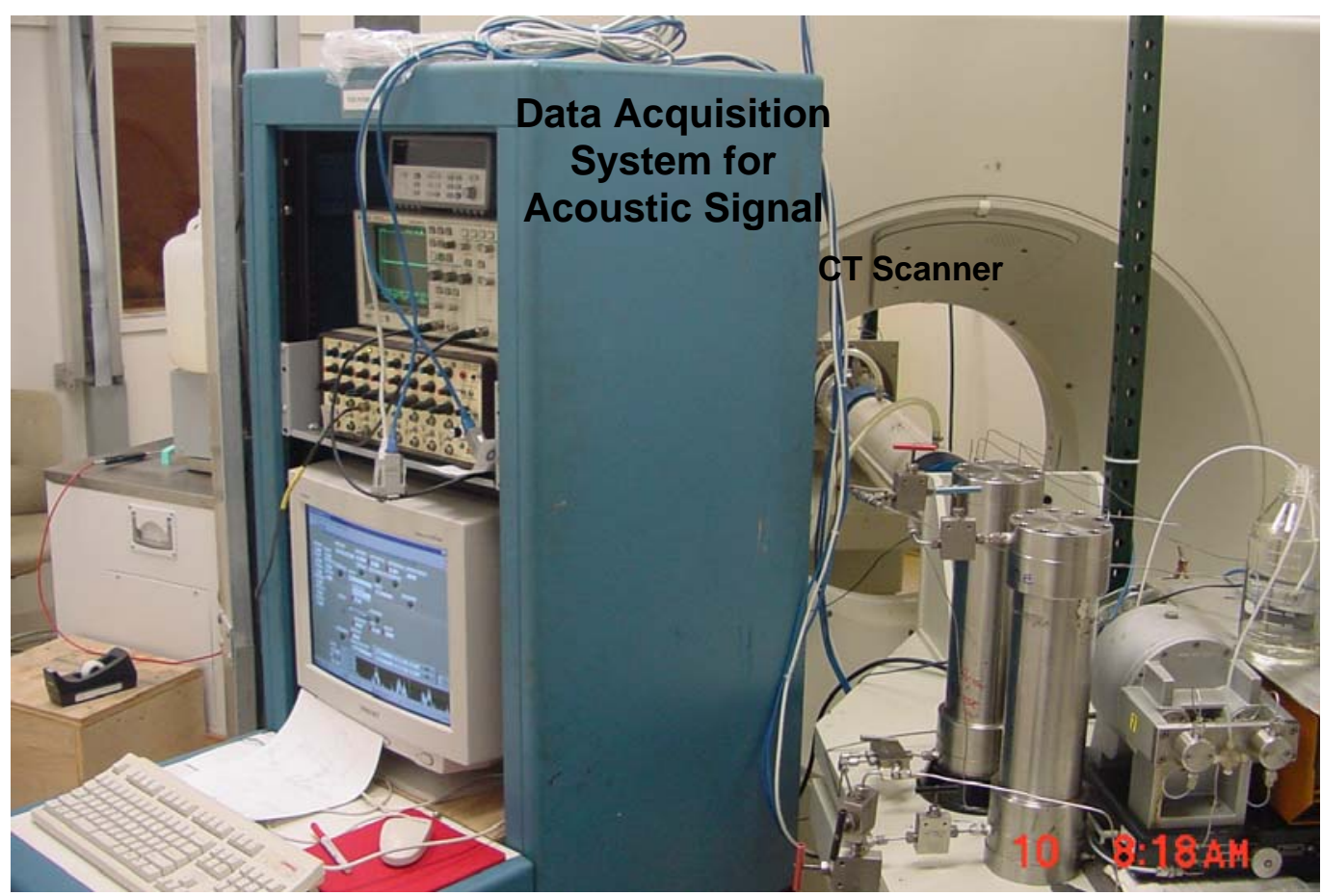

Figure 1.5. Experimental Setup (Back Side) for Hydrate Formation in Core

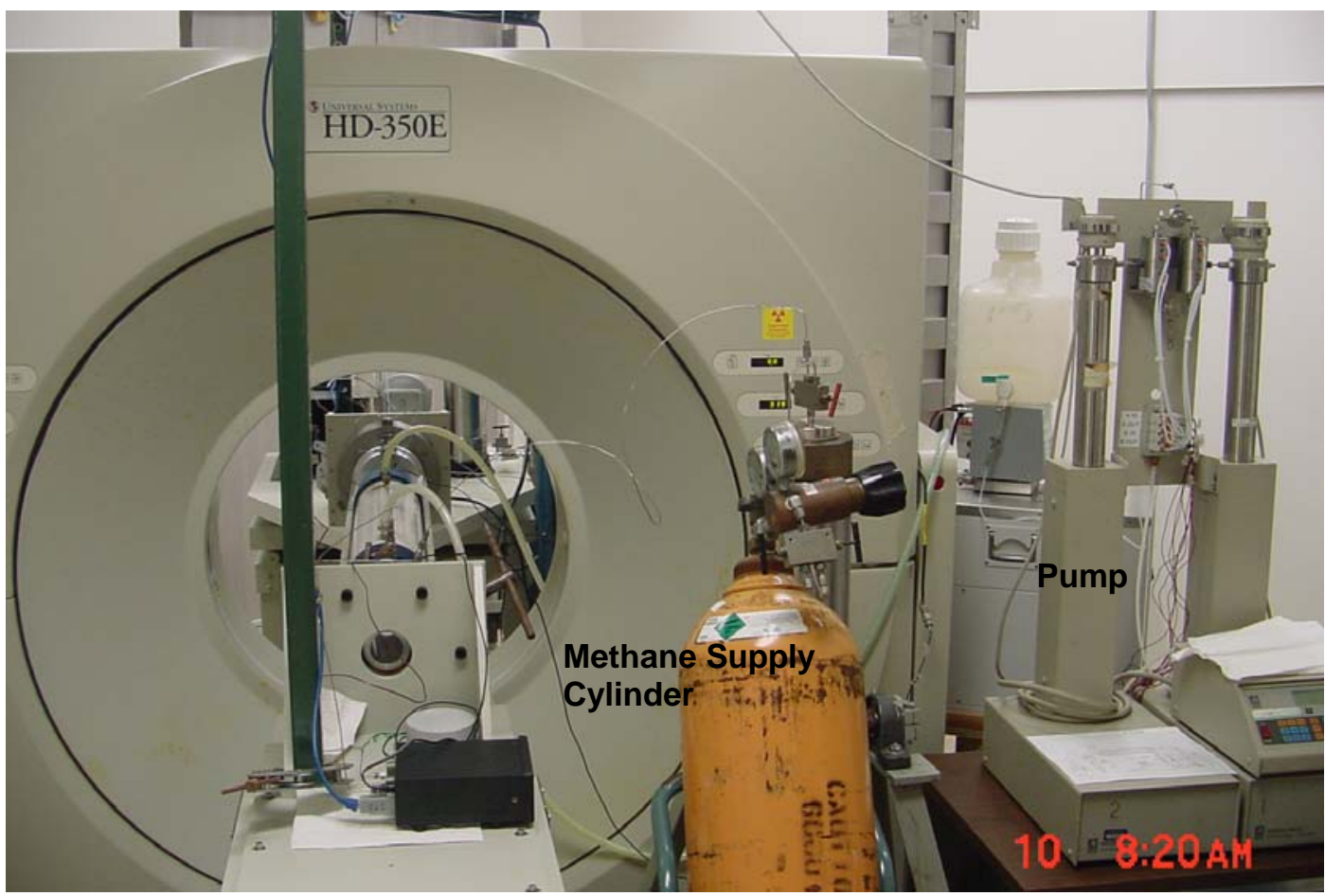




\subsection{MATERIAL CHARACTERIZATIONS}

\subsubsection{Synthetic Core Material Selection}

For application to subsurface formations, core materials of various permeability ranges were selected. Gas hydrates have been discovered in coarse grain sands in the Mackenzie Delta, silts offshore South Carolina and in unconsolidated sands in the Gulf of Mexico. To provide measurements that cover this range, four sets of material were selected, two types of manufactured sandstones (QF-50 and QF-10), Berea sandstone, and an unconsolidated sand pack of known grain size and distribution. The Berea was not used in the hydrate tests. Sample properties and characterization is shown below. Due to the unconsolidated nature of the sand pack, a full characterization was not performed. Sample properties and characterization are shown below.

\subsubsection{Core Properties}

Three core plug samples from materials QF-50, QF-10 and Berea, were submitted for analysis. The samples that were determined to be viable for routine properties testing were trimmed to right cylinders, weighed, extracted with toluene for saturation determination, leached with methanol to remove the salt, and oven dried to stable weights. Helium grain volumes were measured at ambient conditions. Helium pore volume and permeability to nitrogen gas were measured at the minimum confining stress of 800 psi. Table 1.2, following, shows the sample Routine Core Analysis results.

Table 1.1, Summary of Routine Core Analysis Results

\begin{tabular}{|c|c|c|c|c|c|c|c|c|}
\hline \multirow[b]{2}{*}{$\begin{array}{l}\text { Sample } \\
\text { ID }\end{array}$} & \multirow[b]{2}{*}{$\begin{array}{c}\text { Dry } \\
\text { Weight } \\
\text { (g) }\end{array}$} & \multirow[b]{2}{*}{$\begin{array}{c}\text { Calipered } \\
\text { Length } \\
(\mathrm{cm})\end{array}$} & \multirow[b]{2}{*}{$\begin{array}{c}\text { Calipered } \\
\text { Diameter } \\
(\mathrm{cm})\end{array}$} & \multirow[b]{2}{*}{$\begin{array}{c}\text { Grain } \\
\text { Volume } \\
\left(\mathrm{cm}^{3}\right)\end{array}$} & \multirow[b]{2}{*}{$\begin{array}{l}\text { Grain } \\
\text { Density } \\
\left(\mathrm{g} / \mathrm{cm}^{3}\right)\end{array}$} & \multicolumn{2}{|c|}{ Ambient PV $=(L x A)-G V$} & \multirow[b]{2}{*}{$\begin{array}{c}\text { Air } \\
\text { Permeability } \\
\text { (md) }\end{array}$} \\
\hline & & & & & & $\begin{array}{c}\text { Pore } \\
\text { Volume } \\
\left(\mathrm{cm}^{3}\right)\end{array}$ & $\begin{array}{l}\text { Porosity } \\
\text { (percent) }\end{array}$ & \\
\hline Berea & 11.9625 & 1.135 & 2.511 & 4.466 & 2.679 & 1.155 & 20.5 & \\
\hline QF-50 & 9.8877 & 1.309 & 2.488 & 3.718 & 2.660 & 2.646 & 41.6 & \\
\hline QF-10 & 9.7193 & 1.252 & 2.501 & 3.662 & 2.654 & 2.489 & 40.5 & \\
\hline Berea A & 155.3777 & 6.537 & 3.830 & 59.172 & 2.626 & 16.136 & 21.4 & 679 \\
\hline $\begin{array}{c}\text { QF-10 } \\
\text { A }\end{array}$ & 119.0109 & 6.595 & 3.814 & 45.735 & 2.602 & 29.616 & 39.4 & 28170 \\
\hline $\begin{array}{c}\text { QF-50 } \\
\text { A }\end{array}$ & 122.8561 & 6.525 & 3.844 & 47.231 & 2.601 & 28.490 & 37.6 & 78651 \\
\hline Berea B & 150.7061 & 6.384 & 3.815 & 57.336 & 2.628 & 15.639 & 21.6 & 706 \\
\hline $\begin{array}{c}\text { QF-10 } \\
B\end{array}$ & 116.7254 & 6.558 & 3.815 & 44.856 & 2.602 & 30.104 & 40.3 & 29317 \\
\hline $\begin{array}{c}\text { QF-50 } \\
\text { B }\end{array}$ & 122.9031 & 6.486 & 3.863 & 47.599 & 2.582 & 28.419 & 37.4 & 77414 \\
\hline
\end{tabular}

\subsubsection{Geological Characterization}


This study was initiated to evaluate the textural, compositional and pore geometry characteristics of clean Berea sandstone and two quartz synthetic sandstone plug samples. The samples were part of an ongoing porous media research project. This study involved both qualitative and quantitative thin section, $x$-ray diffraction and scanning electron microscope analysis of the samples. The analytical program of this study involved the following elements:

- A thin wafer cut from the end of each core sample was vacuum impregnated with luminescent blue epoxy resin. The resulting sample was thin sectioned and examined with a Zeiss Universal petrographic microscope equipped with an electromechanical stage assembly. Petrographic analysis yielded qualitative information concerning grain size, sorting, composition and cementation. All thin sections were also examined by image analysis techniques yielding quantitative information concerning total porosity and porosity distribution. The results of thin section analyses are presented in Appendix A.

- A portion of each core sample was analyzed by x-ray diffraction techniques (Table 1.2, following). This involved the examination of both powdered bulk rock and oriented $<4$ m size (clay) fraction specimens with a Philips x-ray diffractometer from 2 to 60 degrees 2-theta using APD and ProFit software. The results of x-ray diffraction analyses are presented in Table 1.3, following.

A portion of each core specimen was broken to reveal a fresh surface, cleaned, mounted on an aluminum stub and gold coated. This sample mount was examined with a JEOL 820 scanning electron microscope equipped with an energy dispersive elemental x-ray analysis system (EDS). This form of analysis yields information concerning pore structure, cementation, clay mineralogy and the distribution and morphology of cements. The results of SEM analyses for each sample are presented as a series of black-and-white photomicrographs in Appendix A. 
Table 1.2. X-Ray Diffraction Analysis

\begin{tabular}{|c|c|c|c|c|c|c|c|c|c|c|c|c|c|c|c|c|c|c|c|c|c|c|c|c|c|c|c|c|}
\hline \multirow[b]{2}{*}{ 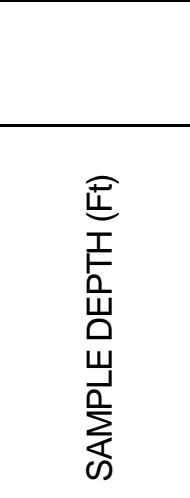 } & \multicolumn{7}{|c|}{ CLAY MINERALS } & \multicolumn{6}{|c|}{ OTHER MINERALS } & \multicolumn{6}{|c|}{$\begin{array}{l}\text { CARBONATE } \\
\text { MINERALS }\end{array}$} & \multicolumn{4}{|c|}{$\begin{array}{c}\text { DRILLING } \\
\text { MUD } \\
\text { SOLIDS }\end{array}$} & \multicolumn{4}{|c|}{ TOTALS } & \multirow[b]{2}{*}{ 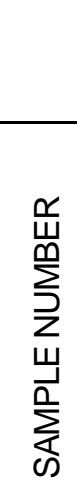 } \\
\hline & $\begin{array}{l}\stackrel{W}{E} \\
\stackrel{W}{W} \\
\sum_{\omega}\end{array}$ & 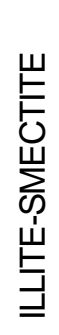 & $\stackrel{⿱ \uplus}{\Xi}$ & $\begin{array}{l}\text { W } \\
\underline{\underline{E}} \\
\underline{\Xi} \\
\underline{\underline{Q}} \\
\underline{\underline{\Sigma}}\end{array}$ & 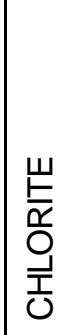 & 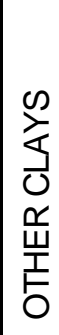 & 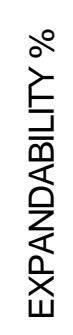 & $\begin{array}{l}\frac{N}{\alpha} \\
\frac{\alpha}{2} \\
O\end{array}$ & 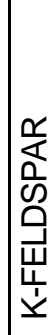 & 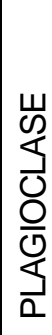 & $\frac{w}{\frac{w}{\underline{\alpha}}}$ & 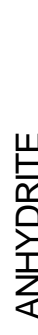 & 誉 & 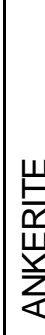 & $\frac{m}{\frac{m}{U}}$ & 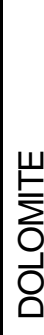 & 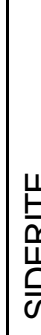 & D. & 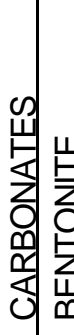 & 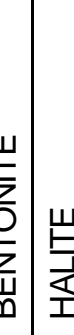 & : & $\begin{array}{l}0 \\
0 \\
0 \\
0 \\
0 \\
0\end{array}$ & 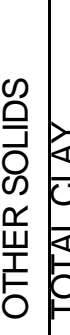 & 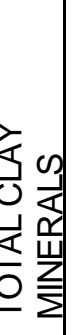 & 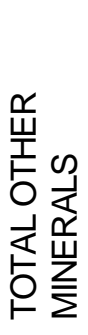 & 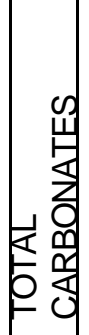 & 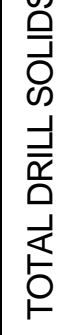 & \\
\hline $\begin{array}{c}\text { Berea } \\
\text { Sandstone }\end{array}$ & & $\operatorname{Tr}$ & 4 & 4 & $\operatorname{Tr}$ & & $\begin{array}{l}25- \\
30 \\
\end{array}$ & 76 & 7 & 3 & & & & & & 6 & & & & & & & & 8 & 86 & 6 & 0 & 003 \\
\hline QF-10 & & & & & & & & 100 & & & & & & & & & & & & & & & & 0 & 100 & 0 & 0 & 002 \\
\hline QF-50 & & & & & & & & 100 & & & & & & & & & & & & & & & & 0 & 100 & 0 & 0 & 001 \\
\hline & & & & & & & & & & & & & & & & & & & & & & & & & & & & \\
\hline
\end{tabular}

Table 1.3. Thin Section Description Summary

\begin{tabular}{|c|c|c|c|c|c|c|c|c|}
\hline $\begin{array}{l}\text { SAMPLE } \\
\text { ID }\end{array}$ & LITHOLOGY & $\begin{array}{l}\text { GRAIN } \\
\text { SIZE } \\
(\mathrm{mm})\end{array}$ & SORTING & GRAIN COMPOSITION & $\begin{array}{c}\text { PORE } \\
\text { FILL/CEMENTS }\end{array}$ & $\begin{array}{c}\text { IMAGE ANALYSIS } \\
\text { POROSITY } \\
(\%)\end{array}$ & PORE TYPES & $\begin{array}{l}\text { SAMPLE } \\
\text { NUMBER }\end{array}$ \\
\hline $\begin{array}{l}\text { Berea } \\
\text { Sandston } \\
\quad \mathrm{e}\end{array}$ & Sandstone & 0.22 & Well & $\begin{array}{c}\text { Monocrystalline quartz }> \\
\text { polycrystalline quartz }> \\
\text { plagioclase feldspar \& K-feldspar } \\
>\text { schistose metamorphic rock } \\
\text { fragments }>\text { volcanic rock } \\
\text { fragments }>\text { muscovite }>\text { epidote }\end{array}$ & $\begin{array}{c}\text { Dolomite }> \\
\text { kaolinite }>\text { silica } \\
\text { overgrowths }> \\
\text { pore lining clay }> \\
\text { siderite }>\text { pyrite }\end{array}$ & 12.95 & $\begin{array}{c}\text { Large } \\
\text { intergranular > } \\
\text { small intergranular } \\
\text { > large secondary } \\
\text { > small secondary } \\
\text { > microporosity }\end{array}$ & 003 \\
\hline QF-10 & Synthetic & 0.21 & Well & Monocrystalline quartz & Resin & 27.53 & $\begin{array}{c}\text { Large } \\
\text { intergranular }\end{array}$ & 002 \\
\hline QF-50 & Synthetic & 0.42 & Moderate & Monocrystalline quartz & Resin & 33.69 & $\begin{array}{c}\text { Large } \\
\text { intergranular }\end{array}$ & 001 \\
\hline
\end{tabular}




\section{Description of the Berea Sandstone}

Lithology-Texture - The sample submitted for analysis was a fine grained, well sorted, moderately compacted sandstone. The mean grain size of the sandstone was $0.22 \mathrm{~mm}$. There was a relatively small standard deviation of framework grain sizes. The rock was devoid of laminar or dispersed depositional shale. Framework sand grains were subangular to subround in shape. Grains experienced rotation into a close packing arrangement in response to burial loading. Compaction was partially responsible for the loss of porosity and permeability experienced by the sandstone during burial.

Grain Composition - The sandstone was moderately quartzose. Monocrystalline quartz was the most abundant framework grain. Polycrystalline quartz was much less abundant. The rock contained smaller quantities of feldspars and rock fragments. Feldspars were largely plagioclase. Rock fragments were dominantly schistose metamorphic varieties. Volcanic igneous rock fragments were less abundant. Accessory grains included deformed ductile micas (muscovite squeezed between framework grains) and the heavy mineral epidote.

Cement Composition - Intergranular pore space was partially occluded by secondary cements. Pores and pore throats were partially occluded by small silica overgrowths developed on framework quartz grains. Dolomite cement was common, occurring as coarsely crystalline pore fill cement in patches throughout the rock. Scattered pores were lined by growths of clay cement and partially filled by bookletlike aggregates of kaolinite clay. The rocks contained small amounts of siderite and pyrite cement.

Nature and Distribution of Shale - The sandstone contained small amounts of shale ( $8 \%)$. The rock was devoid of laminar or dispersed depositional shale. The rock lacked structural shale rock fragments. The shale component of the rock consisted of pore lining and pore filling clay cement. The pore lining clay consisted of illite. The pore filling clay consisted of kaolinite.

Porosity-Porosity Distribution - The core sample submitted for analysis had $12.95 \%$ image analysis porosity. The bulk of the porosity was primary intergranular macroporosity. Image analysis revealed that three (3) overlapping normal distributions of intergranular pore sizes existed with mean values of 30, 50 and $75 \mu \mathrm{m}$. These were interconnected through pore throats less than $1 \mu \mathrm{m}$ in diameter. The rock also contained smaller volumes of secondary dissolution macro and mesopores created by dissolution of framework feldspar grains. The larger secondary pores had diameters of 100-150 $\mu \mathrm{m}$. Microporosity (pores $\ll 5 \mu \mathrm{m}$ diameter) existed in association with leached grains and with clay cements (primarily kaolinite). 


\section{Description of the Synthetic Sandstones}

Lithology-Texture - Two synthetic sandstones were submitted for analysis (QF-10 and QF-50). These samples consisted of loose sand grains indurated by epoxy resin. The QF-10 sample was fine grained $(0.21 \mathrm{~mm})$ and well sorted. The standard deviation of sand grain sizes was small. Sand grains were subangular to subround in shape. Grains had both tangential and long contacts. Grain rotation into a closer packing arrangement resulted from compactional pressures presumably applied at the time of sample preparation. Compaction/packing effects resulted in some reduction in porosity and permeability compared with a loose sand pack. Grain packing was heterogeneous and some portions of the sample were less compacted than others. The sample was characterized by some partial occlusion of pore space by crushed grain residue.

The QF-50 sample was medium grained. The mean grain size of the sample was $0.42 \mathrm{~mm}$. The sample was moderately sorted reflecting a large standard deviation of grain sizes. Grains were subround to round in shape. Grains had a relatively open packing arrangement compared with the QF-10 or Berea sandstone samples. This resulted in much higher pore volume and permeability compared with these samples.

Grain Composition - Both the QF-10 and QF-50 samples were highly quartzose. The rocks consisted exclusively of quartz sand grains or crushed sand (in the QF-10 sample). There were a few scattered heavy mineral grains (epidote). Thus, the synthetic sandstones were much more quartzose than the Berea sandstone sample.

Cement Composition - The synthetic sandstones were devoid of secondary cements. The rocks were indurated by epoxy resin but lacked natural cements. Some pore space, particularly in the QF-10 sample, was filled by crushed quartz grain residue.

Nature and Distribution of Shale - Neither sample contained shale or clay cement.

Porosity-Porosity Distribution - The QF-10 sample had $27.53 \%$ image analysis porosity. All of the porosity in this sample was intergranular. The sample was devoid of secondary dissolution porosity or microporosity. Very small amounts of mesoporosity (pores $<-30 \mu \mathrm{m}$ ) were detected by image analysis. These small pores were associated with crushed grain residue. Image analysis revealed that the intergranular pores occurred in three (3) overlapping distributions with mean diameters of 150, 225 and $300 \mu \mathrm{m}$. The $300 \mu \mathrm{m}$ pore group was most abundant. Virtually all of the pores in this sample were more than $125 \mu \mathrm{m}$ in diameter. 
The QF-50 sample had 33.69\% image analysis porosity. All of the porosity in this sample was intergranular. The sample was devoid of secondary dissolution porosity or microporosity. The pore structure was essentially trimodal. The rock was virtually devoid of pores less than $90 \mu \mathrm{m}$ in diameter. Intergranular pores were concentrated in two groups; 100-300 $\mu \mathrm{m}$ (2 overlapping distributions with means at 150 and $250 \mu \mathrm{m}$ ) and 350-450 $\mu \mathrm{m}$ (mean $400 \mu \mathrm{m}$ ).

\section{Description of the Synthetic Unconsolidated Sediment}

The sediment samples collected by the ChevronTexaco JIP GOM drilling campaign during the summer of 2005 were described as silt with clay. The only information available at the time was that the particle sizes were smaller than $0.063 \mathrm{~mm}(63 \mu \mathrm{m})$. The particle size distribution of similar sub sea sediments reported by Silva, et. al. (1996) was:

Table 1.4, Typical Unconsolidated Sediment

\begin{tabular}{|l|c|c|}
\hline Description & $\begin{array}{c}\text { Particle Size } \\
(\boldsymbol{\mu m})\end{array}$ & $\begin{array}{c}\text { Distribution } \\
(\text { Wt. \%) }\end{array}$ \\
\hline Sand & $>62$ & 3 \\
\hline Silt & $2-62$ & 55 \\
\hline Clay & $<2$ & 42 \\
\hline
\end{tabular}

A sand pack having a particle size over the range of the typical sediment was prepared using naturally occurring microcrystalline silica, finely ground. The sand pack was composed of a 50/50 mixture by weight of the following materials:

Table 1.5, Synthetic Unconsolidated Sediment

\begin{tabular}{|l|c|c|c|c|}
\hline Sample No. & $\begin{array}{c}\text { Particle Size } \\
(\boldsymbol{\mu m})\end{array}$ & $\begin{array}{c}\text { Distribution } \\
(\text { Wt. \%) }\end{array}$ & $\begin{array}{c}\text { Density } \\
(\mathbf{g} / \mathbf{m L})\end{array}$ & $\begin{array}{c}\text { Sediment } \\
\text { Composition (Wt. \%) }\end{array}$ \\
\hline 342890 & 45 & 99.6 & 2.5 & 50 \\
\hline S5631 & $0.5-10$ & 99 & 2.5 & 50 \\
& $1-5$ & 80 & & \\
\hline
\end{tabular}

The above compare well with grain size distributions of the GOM sediment samples collected by the ChevronTexaco JIP, as reported by Hippe, et. al. (2006). The report indicated that approximately 10 
percent of the particles were $30 \mu \mathrm{m}$ or greater, 25 percent were $10 \mu \mathrm{m}$ or greater, and about 50 percent were $1.5 \mu \mathrm{m}$ or greater.

\section{Mercury Injection Capillary Pressure}

Three (3) samples were selected for Injection measurement. The maximum mercury injection pressure was 60,000 psi. Each sample was sub-cored from the plug sample, cleaned with cycles of toluene and methanol, oven dried and permeability and porosity measured at the minimum net confining stress.

The samples were placed into the Micromeretics Autopore IV instrument and air removed by vacuum. Mercury was introduced into the penetrometer and a 1.0-psia pressure was applied. The mercury was drained from the system except for the mercury inside the penetrometer. Air pressure was applied to the mercury in steps up to 35 psia. At each step, mercury was allowed to intrude until equilibrium conditions were achieved. At $35 \mathrm{psia}$, the pressure was lowered to atmospheric (14.7 psia) and the penetrometer transferred to the high pressure cylinder. Air was removed from the cylinder by mineral oil. The penetrometer and sample remained as before. Oil pressure was then applied to the mercury in steps up to 60,000 psia.

A summary plot of the capillary pressure data is included in Figure 1.6. The pore size distribution results are shown in Figure 1.7. Individual results and data are presented in Appendix A. 
Figure 1.6. Mercury Injection Capillary Pressure Results

\section{Mercury Injection Capillary Pressure}

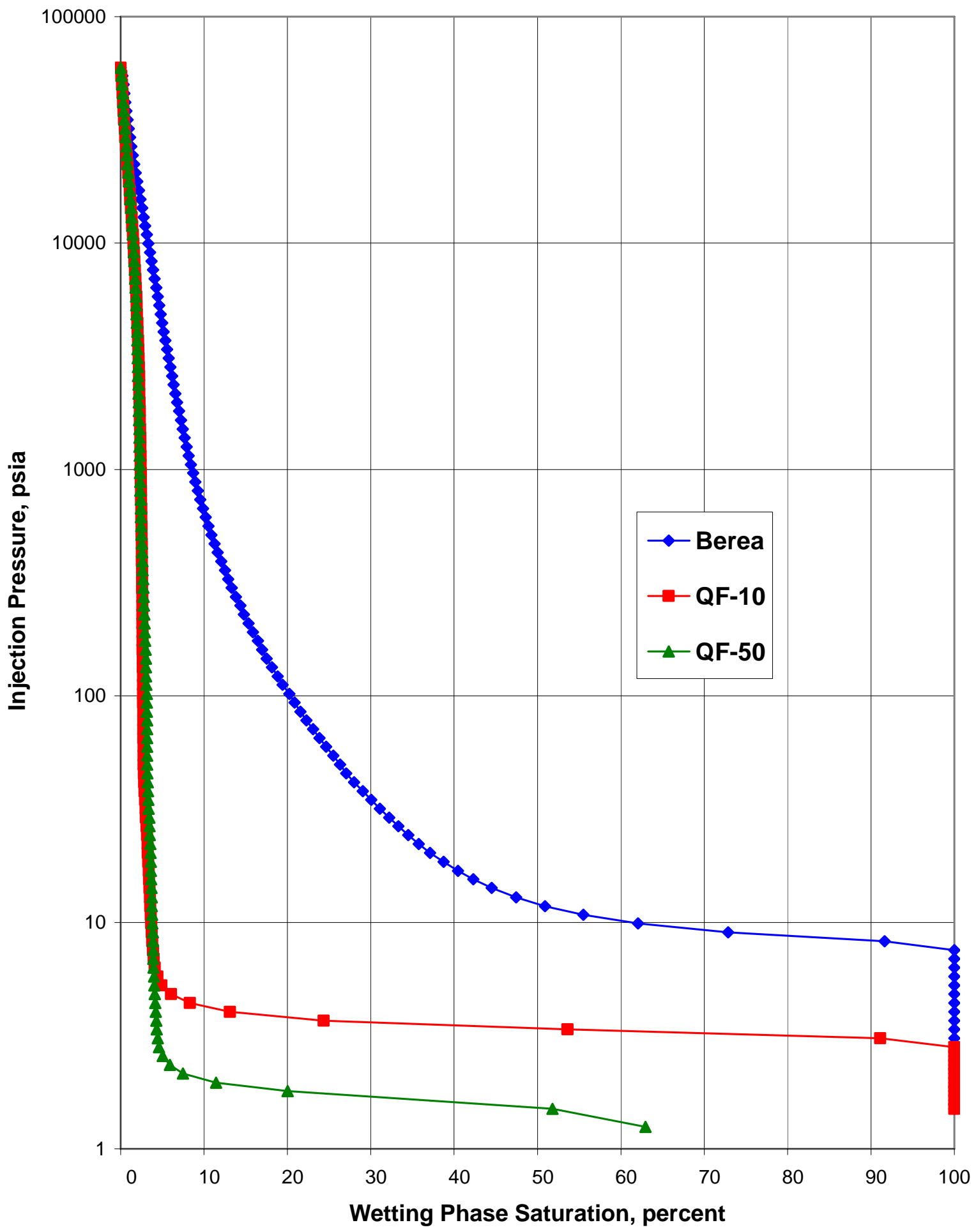


Figure 1.7. Pore Size Distribution Results

\section{Mercury Injection Capillary Pressure}

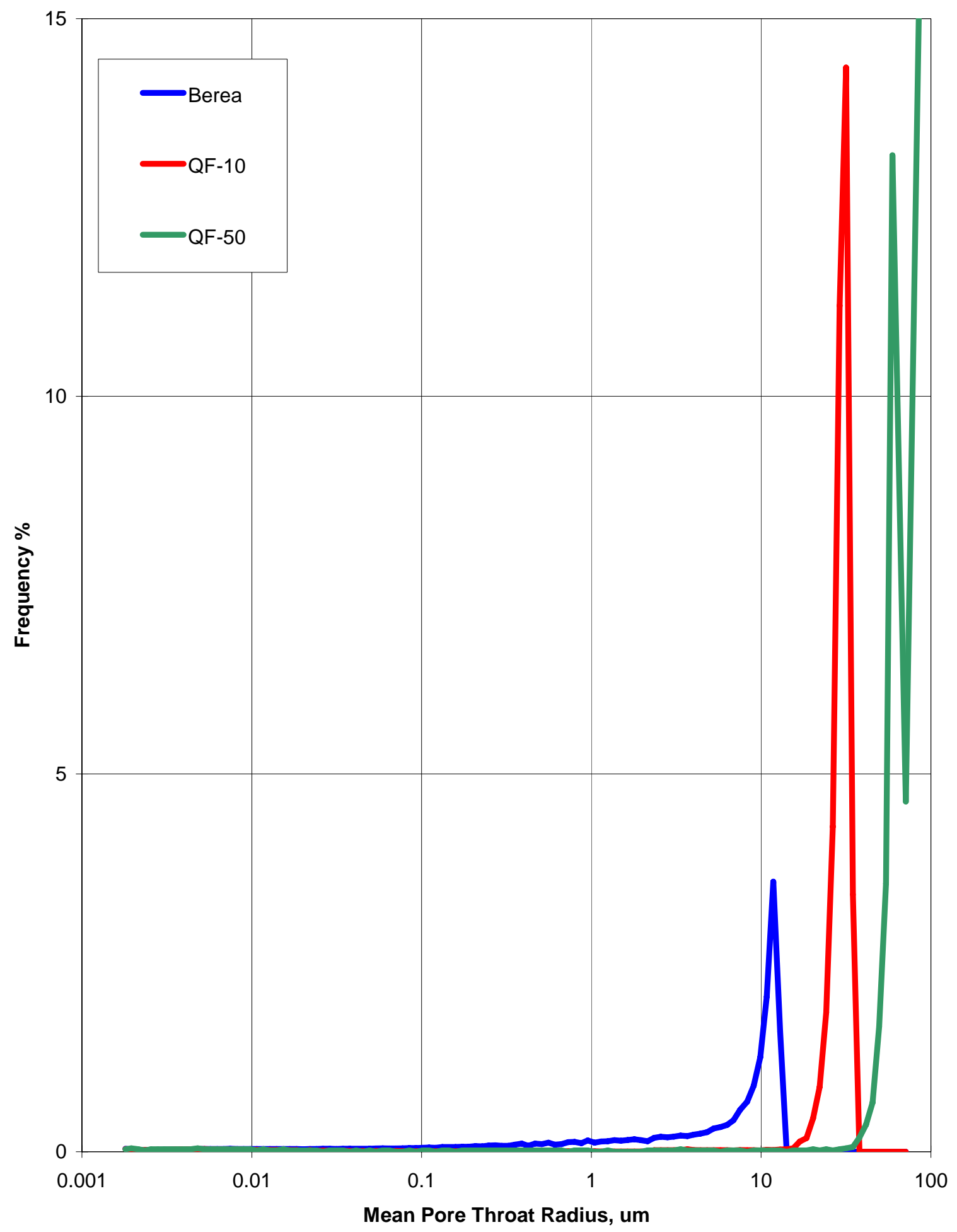




\subsubsection{Geomechanical Properties}

The purpose of this part of the study was to determine the mechanical properties, such as Young's modulus, Poisson's ratio and compressive strength of the core to be used for hydrate formation. The properties were measured under the same pressure regime that was to be used for the hydrate formation in the core. All samples used were saturated using $6 \% \mathrm{Nal}$ solution. Acoustic velocities through the samples were also measured during the test at multiple stress conditions. These data were collected to provide a good understanding of the mechanical behavior of the cores under the hydrate formation stress conditions.

\section{Mechanical Tests}

The plugs used for the study were 1.5 inch in diameter and 3.0 inch in length. The ends of the plugs were trimmed and polished so that the parallelism between ends was within a standard tolerance of 0.001 inch. The length and diameter of each sample was then measured. The samples were then saturated with $6 \% \mathrm{Nal}$ solutions using centrifuge technique and stored in a $6 \% \mathrm{Nal}$ environment.

Steps and procedure used for the Un-Confined test

1. The sample was placed in a thin rubber sleeve using the vacuum sleeve expander apparatus.

2. The sample was then mounted on the bottom platen of the un-confined test vessel.

3. The circumferential band was then placed on the sample for radial deformation measurements.

4. The top platen was mounted on the sample and the vessel was placed in the MTS load frame.

5. The MTS load cell was then brought in contact with the top platen of the test vessel and an initial pressure of 50 psi was applied on the sample.

6. At this time the data acquisition (DAQ) was initiated. The axial strain and stress on the sample were measured using the MTS LVDT and load cell.

7. The sample was held at the initial stage of 50 psi until the stresses and strains reached equilibrium (15 min).

8. At this point the axial stress on the sample was increased using an axial displacement ramp of $0.025 \mathrm{~mm} / \mathrm{min}$.

9. The sample was ramped until failure occurred.

10. The stresses and deformation on the sample were recorded during the test and reported.

Steps and procedure used for the Tri-axial test 
1. The sample was placed in a thin rubber sleeve using the vacuum sleeve expander apparatus.

2. The sample was then mounted on the bottom platen of the Tri-axial test vessel.

3. The circumferential band was then placed on the sample for radial deformation measurements.

4. The top platen is mounted on the sample and the vessel is placed in the MTS load frame.

5. The MTS load cell was then brought in contact with the top platen of the test vessel.

6. The axial and confining stress on the sample was brought to an initial stress of 100psi and, pore pressure was brought to an initial pressure of $50 \mathrm{psi}$.

7. At this time the data acquisition (DAQ) was initiated. The axial, confining and pore pressure data along with axial \& radial strain data were collected. The axial strain was measured using MTS LVDT system.

8. The sample was held at the initial stress condition until the stresses and strains reached equilibrium (15-30 min).

9. The axial and radial stress was then ramped to 3,000 psi and pore pressure to 2,500 psi in 20 minutes.

10. The sample was held at this stress condition for about 15-30 min for the stresses and strains to reach equilibrium.

11. At this point the axial stress on the sample was increased using an axial displacement ramp of $0.025 \mathrm{~mm} / \mathrm{min}$, while keeping both the pore and confining pressure constant.

12. The sample was ramped until failure occurred.

13. The acoustic velocity through the sample was recorded at multiple pressure points during the axial failure displacement ramp.

14. The stresses and deformation on the sample were recorded along with the acoustic velocity, during the test and reported.

Steps and procedures used for the Pore Pressure Draw Dawn test:

1. The sample was placed in a thin rubber sleeve using the vacuum sleeve expander apparatus.

2. The sample was then mounted on the bottom platen of the Tri-axial test vessel

3. The circumferential band was then placed on the sample for radial deformation measurements.

4. The top platen was mounted on the sample and the vessel was placed in the MTS load frame.

5. The MTS load cell was then brought in contact with the top platen of the test vessel.

6. The axial and confining stress on the sample was brought to an initial stress of 100 psi and, pore pressure was brought to an initial pressure of 50 psi. 
7. At this time the data acquisition (DAQ) was initiated. The axial, confining and pore pressure data along with axial and radial strain data were collected. The axial strain was measured using MTS LVDT system.

8. The sample was held at the initial stress condition until the stresses and strains reached equilibrium (15-30 min).

9. The axial and radial stress was then ramped to 3,000 psi and pore pressure to 2,500 psi in 20 minutes.

10. The sample was held at this stress condition for about 15-30 min for the stresses and strains to reach equilibrium.

11. At this point the pore pressure on the sample was decreased at a rate of $50 \mathrm{psi} / \mathrm{min}$ till the sample reached the initial pore pressure conditions of $50 \mathrm{psi}$. During the pressure draw dawn, both the confining and axial stress (overburden) were kept constant.

12. The acoustic velocity through the sample was recorded at multiple pressure points during the pore pressure draw down, along with the axial and radial deformation.

13 The stresses and deformation on the sample were recorded along with the acoustic velocity, during the test and reported.

\section{Data Analysis}

The Young's Modulus, Poisson's ratio and compressive strength of the samples were calculated from the stress-strain curves of the data as described below.

The Young's modulus was calculated from the slope of the axial stress vs. axial strain curve. The Poisson's ratio was calculated from the slope of the axial-strain vs. radial-strain curve. The compressive strength of the sample was the stress where the slope of the axial-strain vs. axial-stress curves changed.

\section{Data Explanation}

The Young's modulus was the measure of elasticity of the sample. The Young's modulus plot (axial strain vs. axial stress plot) had three distinct features (slope), initially at low stress when the sample was reaching equilibrium. The sample experienced a plastic deformation, where the microscopic grain surfaces in the sample were compressed. Then as the sample was stressed, the sample experienced an elastic deformation with a significant higher stress-strain slope compared to initial equilibrium slope. As the sample stress reached its compressive strength, the sample again approached a plastic deformation mode where the stress-strain slope was less than that of the elastic mode. The radial deformation of the sample also followed the same route except that in un-confined test there was no confining stress and the sample did not experience any initial plastic deformation.

Test relation to the project: 
The un-confined, unsaturated test provided the base mechanical property of the core.

The tri-axial test explained how the sample behaved when confining and pore pressures were applied to the sample. This test provided the information about the behavior about matrix structure of the sample, whether the confining and pore stresses made the sample strong, weak or had no effect.

The pore pressure draw down test directly related to the effect on the core (formation) strength as the hydrates were produced, at a given over burden pressure condition.

Table 1.7 Summary of Unconfined Compressive Strength Tests

\begin{tabular}{|c|c|c|c|c|c|c|}
\hline Sample ID & $\begin{array}{c}\text { Sample } \\
\text { Condition }\end{array}$ & $\begin{array}{c}\text { Confining } \\
\text { Pressure } \\
\text { (psi) }\end{array}$ & $\begin{array}{c}\text { Pore } \\
\text { Pressure } \\
\text { (psi) }\end{array}$ & $\begin{array}{c}\text { Failure } \\
\text { Stress (psi) }\end{array}$ & $\begin{array}{c}\text { Young's } \\
\text { Modulus } \\
\text { (psi) }\end{array}$ & $\begin{array}{c}\text { Poisson's } \\
\text { Ratio } \\
\text { (mm/mm) }\end{array}$ \\
\hline QF-50 C & dry & 0 & 0 & 3300 & $6.80 \mathrm{E}+05$ & 0 \\
\hline QF-50 D & dry & 0 & 0 & 3100 & $7.80 \mathrm{E}+05$ & 0.19 \\
\hline QF-50 E & dry & 0 & 0 & 3300 & $7.20 \mathrm{E}+05$ & 0 \\
\hline QF-10 C & dry & 0 & 0 & 8700 & $1.01 \mathrm{E}+06$ & 0.18 \\
\hline Berea C & dry & 0 & 0 & 4500 & $1.00 \mathrm{E}+06$ & 0.27 \\
\hline Berea D & dry & 0 & 0 & 4000 & $8.40 \mathrm{E}+05$ & 0.18 \\
\hline Berea E & dry & 0 & 0 & 4700 & $1.20 \mathrm{E}+06$ & 0.19 \\
\hline
\end{tabular}


Table 1.8 Summary of Acoustic Properties Data

\begin{tabular}{|c|c|c|c|c|c|c|c|c|c|c|c|c|}
\hline Test & Sample & Displacement & Dia & $\mathbf{L}$ & Paxial & Ppore & Pconf & $d X$ & Tp & Ts & $V p$ & Vs \\
\hline & ID & $\mathrm{mm}$ & inch & inch & psi & psi & psi & inch & usec & usec & $\mathrm{m} / \mathrm{s}$ & $\mathrm{m} / \mathrm{s}$ \\
\hline Set1 & QF50-2 & 39.7574 & 1.5 & 3 & 3963.0 & 2500 & 3000.0 & 0.0000 & 80.50 & 174.50 & 2193 & 929 \\
\hline Set1 & QF50-2 & 40.0997 & 1.5 & 3 & 6510.0 & 2500 & 3000.0 & 0.0135 & 79.50 & 174.00 & 2248 & 931 \\
\hline Set1 & QF10-2 & 40.704 & 1.5 & 3 & 3057.0 & 2500 & 3000.0 & 0.0000 & 68.50 & 136.00 & 3349 & 1752 \\
\hline Set1 & QF10-2 & 41.1055 & 1.5 & 3 & 7416.0 & 2500 & 3000.0 & 0.0158 & 68.25 & 135.75 & 3369 & 1753 \\
\hline Set1 & Berea-2 & 41.7352 & 1.5 & 3 & 6794.0 & 2500 & 3000.0 & 0.0000 & 78.80 & 119.00 & 2306 & 2875 \\
\hline Set1 & Berea-2 & 41.88 & 1.5 & 3 & 8492.0 & 2500 & 3000.0 & 0.0057 & 78.50 & 118.00 & 2322 & 2983 \\
\hline Set2 & QF50-3 & 40.66 & 1.54 & 3.02 & 4529 & 2500 & 3000 & 0.0000 & 82.00 & 190.00 & 2116 & 787 \\
\hline Set2 & QF50-3 & 40.818 & 1.54 & 3.02 & 5661 & 2500 & 3000 & 0.0062 & 82.00 & 189.50 & 2112 & 789 \\
\hline Set2 & QF10-3 & 39.68 & 1.5 & 3.01 & 3170 & 2500 & 3000 & 0.0000 & 78.25 & 129.50 & 2352 & 2066 \\
\hline Set2 & QF10-3 & 40.04 & 1.5 & 3.01 & 6794 & 2500 & 3000 & 0.0142 & 78.00 & 130.25 & 2360 & 2016 \\
\hline Set2 & Berea-4 & 40.65 & 1.5 & 3 & 4642 & 2500 & 3000 & 0.0000 & 79.80 & 135.00 & 2238 & 1793 \\
\hline Set2 & Berea-4 & 40.88 & 1.5 & 3 & 6794 & 2500 & 3000 & 0.0091 & 79.00 & 134.00 & 2285 & 1831 \\
\hline set3 & QF50-4 & 41.2238 & 1.53 & 2.99 & 4529 & 2500 & 3000 & 0.0000 & 79.75 & 146.50 & 2234 & 1406 \\
\hline set3 & QF50-4 & 41.3844 & 1.53 & 2.99 & 5661 & 2500 & 3000 & 0.0063 & 79.50 & 146.25 & 2245 & 1410 \\
\hline set3 & QF10-5 & 40.89 & 1.49 & 2.97 & 6397 & 2500 & 3000 & 0.0000 & 68.75 & 147.25 & 3280 & 1378 \\
\hline set3 & QF10-5 & 41.06 & 1.49 & 2.97 & 8492 & 2500 & 3000 & 0.0067 & 68.75 & 147.15 & 3273 & 1377 \\
\hline set3 & Berea-5 & 51.015 & 1.5 & 2.6 & 5095 & 2500 & 3000 & 0.0000 & 76.50 & 122.00 & 2148 & 2239 \\
\hline set3 & Berea-5 & 51.15 & 1.5 & 2.6 & 6794 & 2500 & 3000 & 0.0053 & 76 & 121 & 2179 & 2312 \\
\hline
\end{tabular}




\subsection{FLUID SYSTEM}

Methane gas and brine were used for hydrate formation in the core samples. The brine was a 6 -wt $\%$ Sodium lodide ( $\mathrm{Nal}$ ) and de-ionized water mixture. The gas was 100\% methane. A hydrate equilibrium curve was experimentally measured in a blind cell for three concentrations Nal solution prepared separately in DI water and potable water after reverse osmosis. The equilibrium graph is shown in Figure 1.8. This indicates that the effect of sodium iodide concentration could be in the order of 600 psi.

Figure 1.8. Gas Hydrate Equilibrium for Methane 6-wt\% Nal Brine

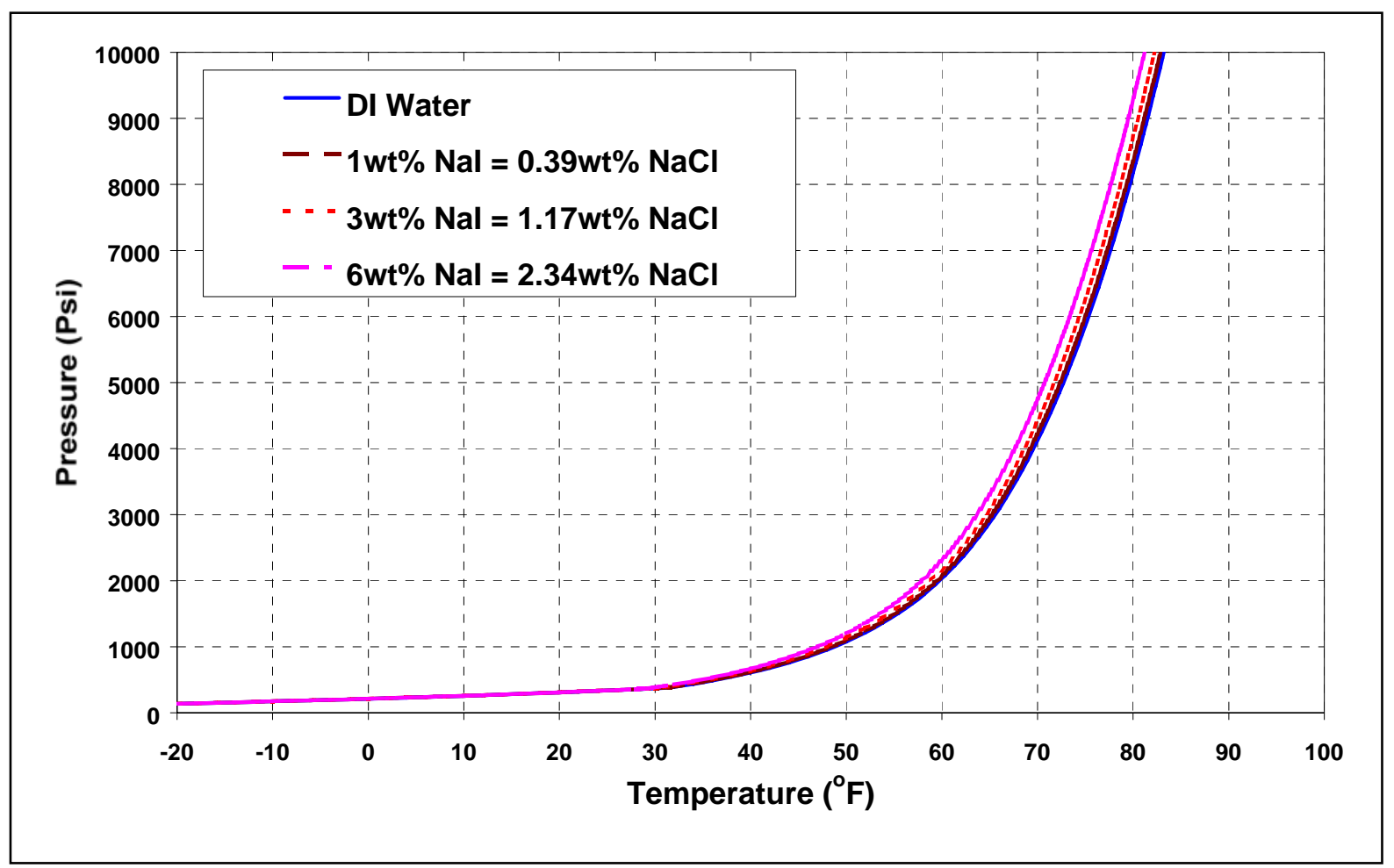




\subsection{SYSTEM TEST}

Prior to the formation of hydrates in core samples, a test to measure ice formation temperature for 6 -wt $\%$ $\mathrm{Nal}$ solution at 2,500 psia was conducted. The purpose of the test was to ensure that ice formation occurred at a much lower temperature than hydrate formation temperature. Additionally, the ice formation test also allowed for measurement of the ice CT number under experimental conditions that were used for calculation of hydrate formation in the core. At 2,500 psia, 6-wt\% Nal solution started forming ice at $\sim 22^{\circ} \mathrm{F}$ which was much lower than the hydrate formation temperature for the same solution. The results are shown in Figure 1.9.

Figure 1.9. Sodium lodide Ice Formation

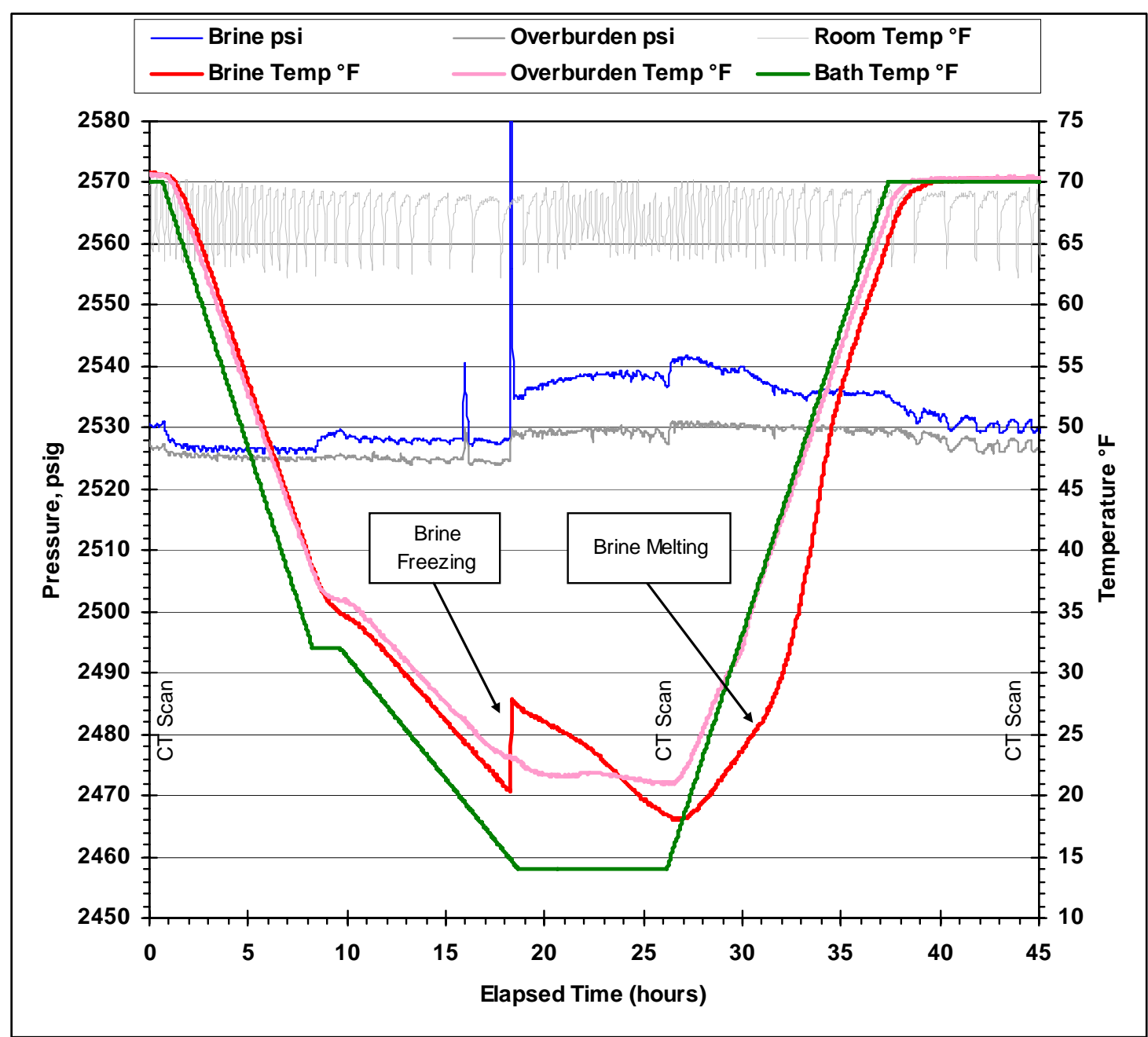


In an initial experiment, a cell was cooled to $30^{\circ} \mathrm{F}$ at 3,000 psia for a system test which was successful. An experimental cell was half filled with 6 -wt\% sodium iodide solution ( $\mathrm{Nal}$ ) at 3,000 psia. The other half of the experimental cell was filled with methane gas. The system was cooled to $40^{\circ} \mathrm{F}$ and a base CT scan near the middle of the cell was acquired. The system temperature was maintained at $40^{\circ} \mathrm{F}$ for the next 4-days. A CT scan at the location of the base scan was acquired each day for the next four days. By subtracting the base CT scan from individual scans acquired over the four days, a hydrate layer was clearly detected at the brine-methane interface.

Figures 1.10 to 1.14 , following, show the subtraction images and width of the hydrate layer formed. Figure 1.10 shows the base scan on day zero. Figures 1.11 through 1.14 show the successive base scans over the next four days. In Figures 1.15 through 1.18, the base scan from day zero was subtracted from each scan, and the resultant images indicate that the hydrate layer grows as a function of time.

The contrast of colors in the CT images was generated by differences in X-ray absorption that arose principally from differences in density within the cross section. Higher CT numbers indicate greater X-ray absorption and higher relative density. The scale of CT numbers versus colors is shown at the bottom of the pictures. 
Figure 1.10. Base CT Scan at $40^{\circ} \mathrm{F}$

Semi-circle Represents 6-wt\% Nal Solution

Methane Gas Occupies other Half of Circle

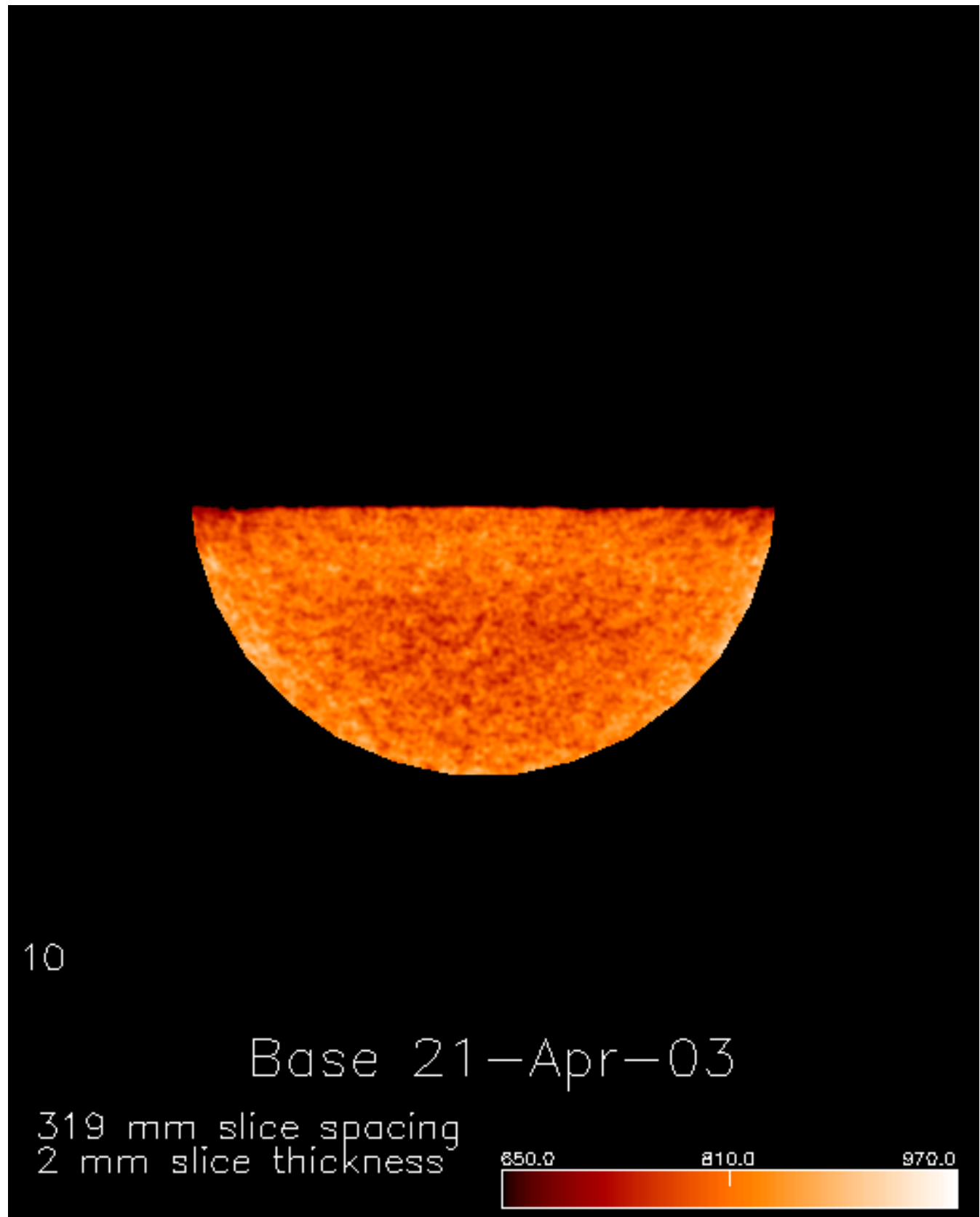


Figure 1.11. First CT Scan at $40^{\circ} \mathrm{F}$ - One Day after Base Scan

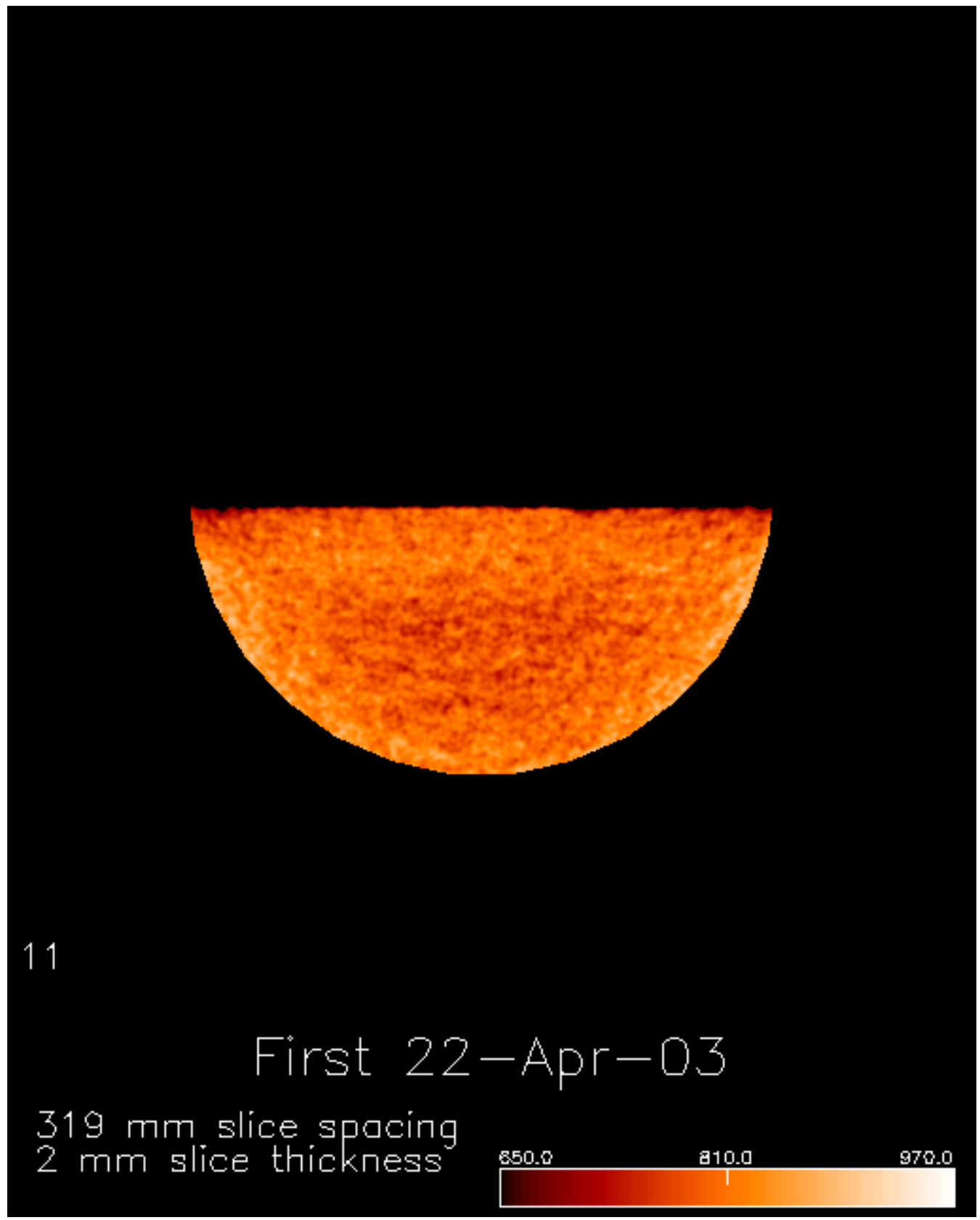


Figure 1.12. Second CT Scan at $40^{\circ} \mathrm{F}$ - Two Days after Base Scan

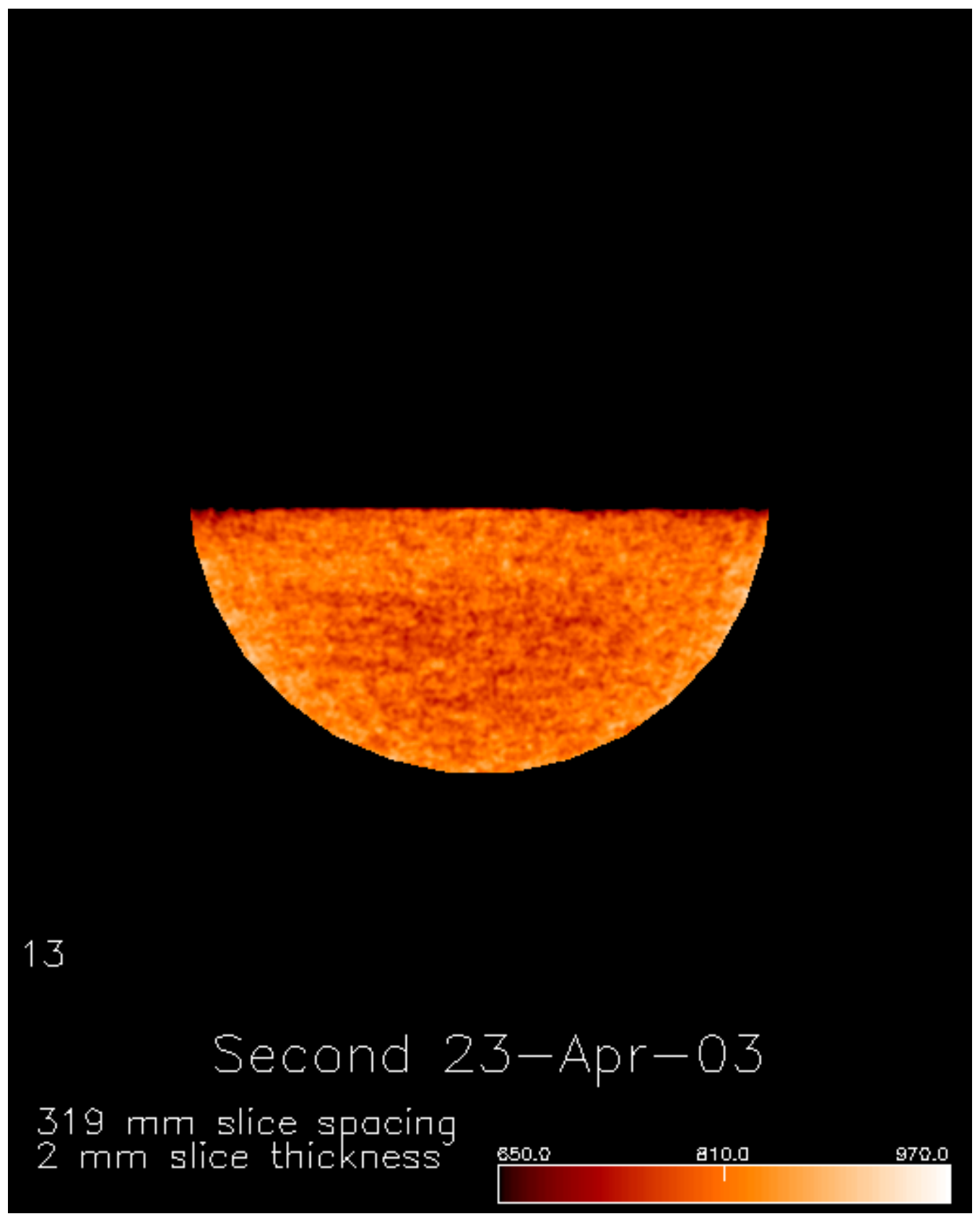


Figure 1.13. Third CT Scan at $40^{\circ} \mathrm{F}$ - Three Days after Base Scan

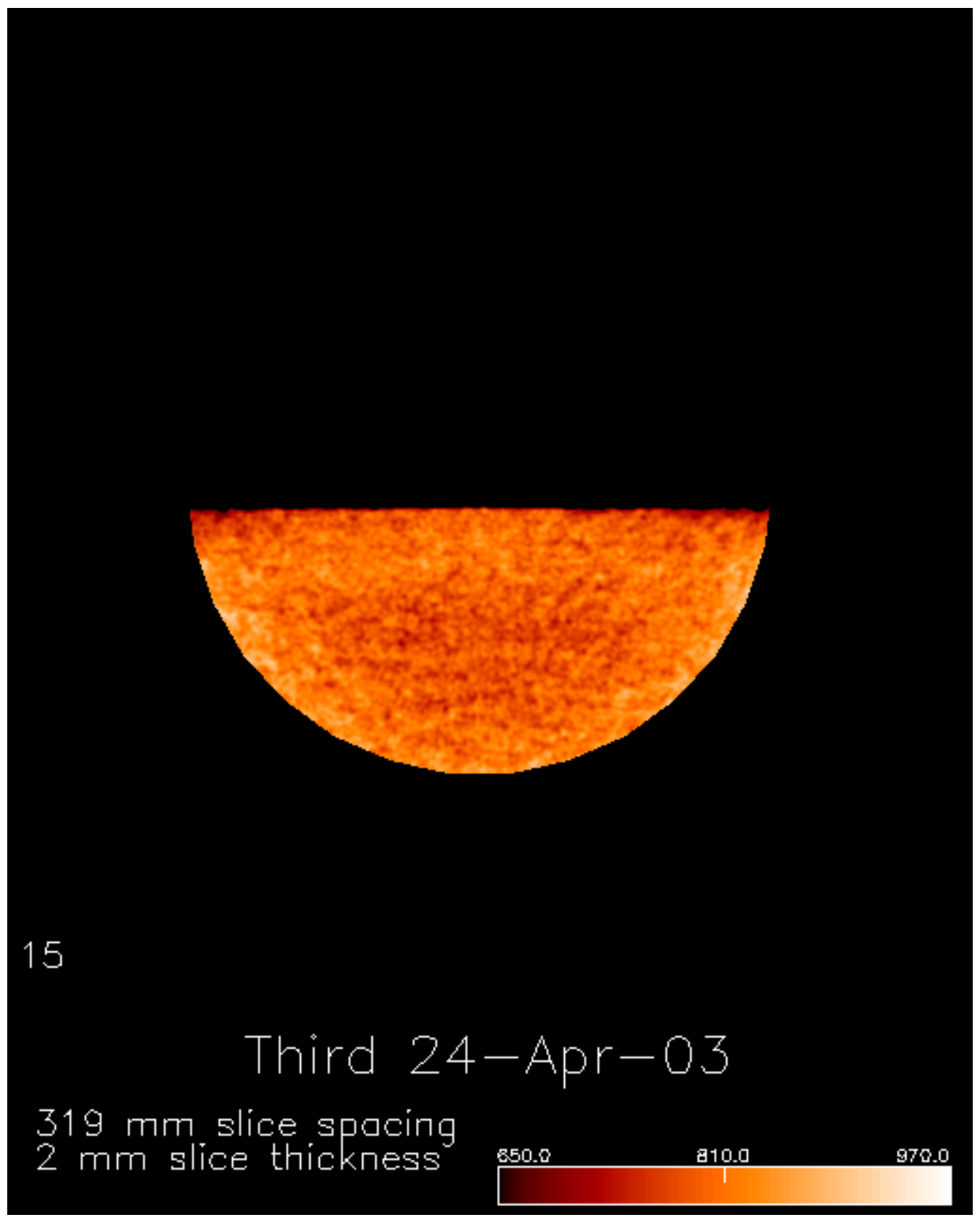


Figure 1.14. Fourth CT Scan at $40^{\circ} \mathrm{F}$ - Four Days after Base Scan

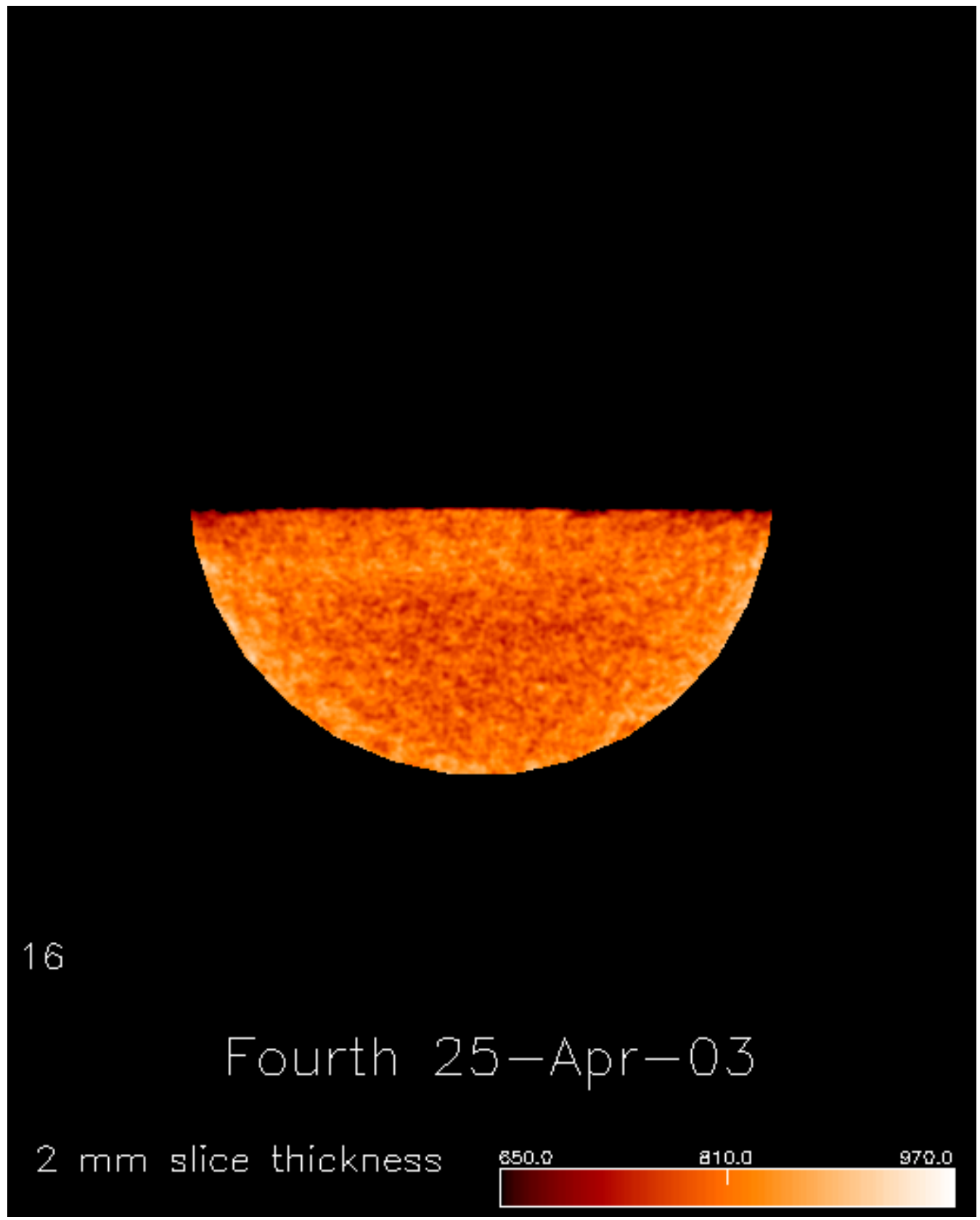


Figure 1.15. Base Scan Subtracted from First Scan White Layer Seen in the Image with L-R Marking is the Hydrate Layer
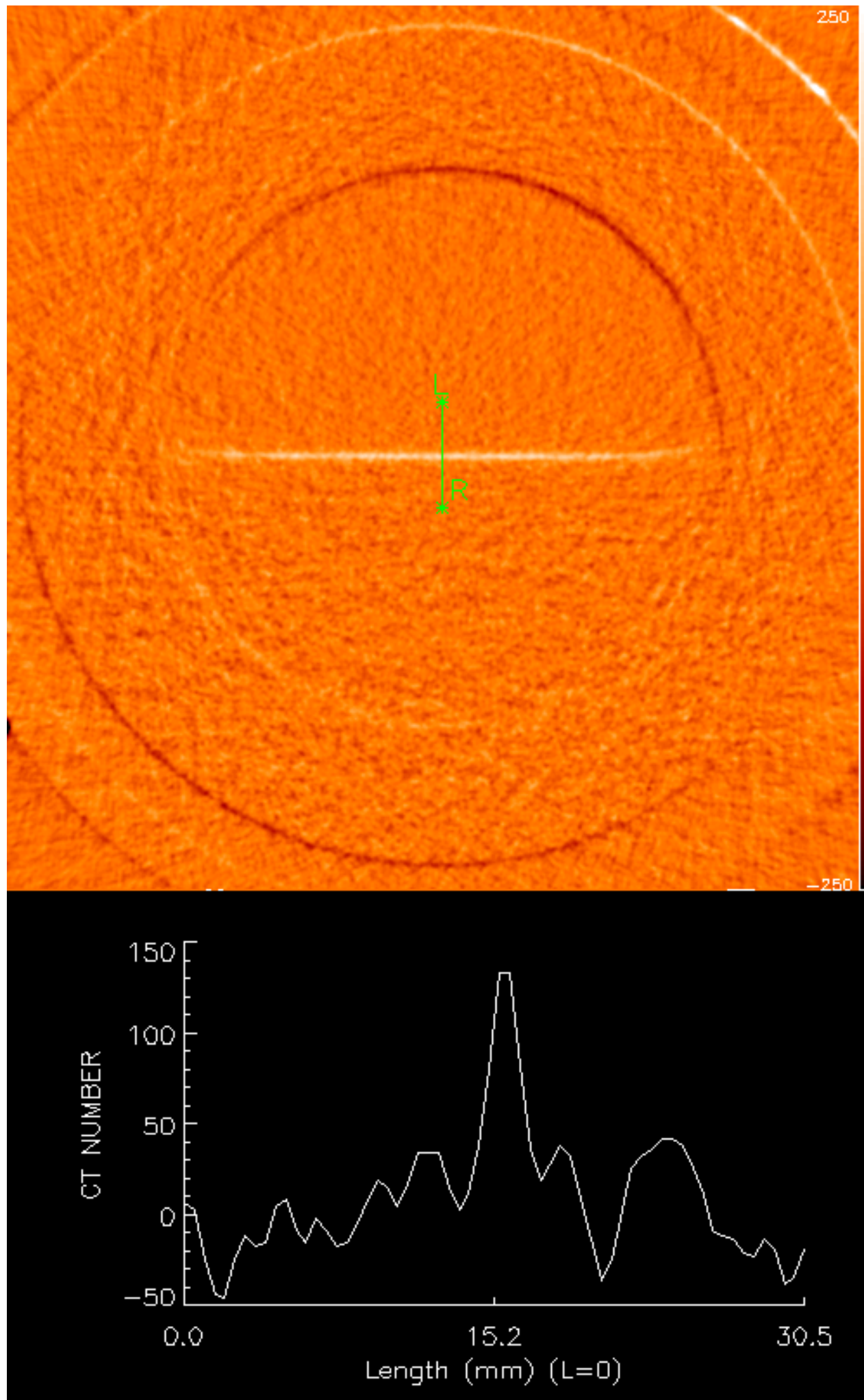
Figure 1.16. Base Scan Subtracted from Second Scan White Layer Seen in the Image with L--R Marking is the Hydrate Layer
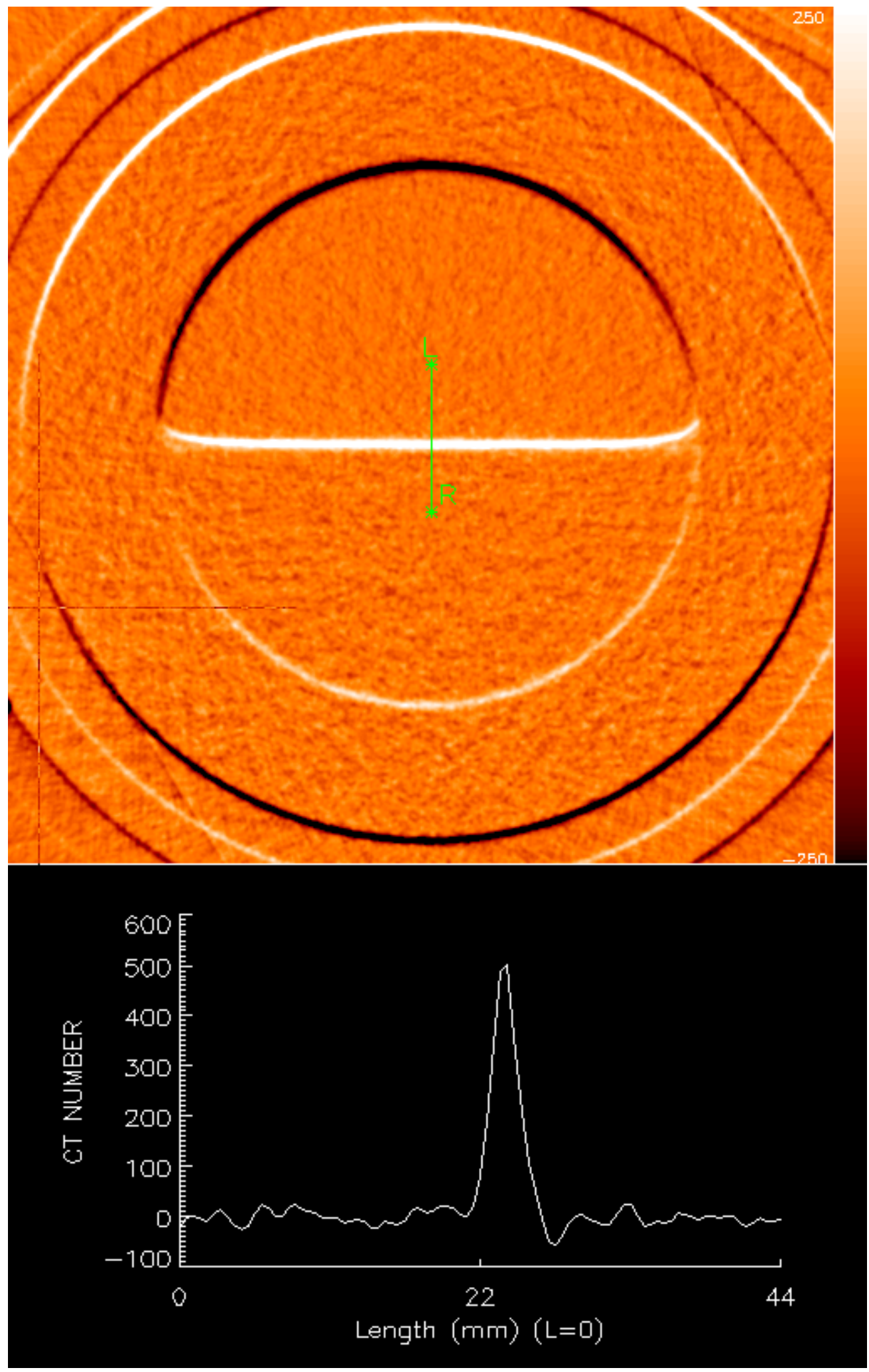
Figure 1.17. Base Scan Subtracted from Third Scan White Layer Seen in the Image with L-R Marking is the Hydrate Layer

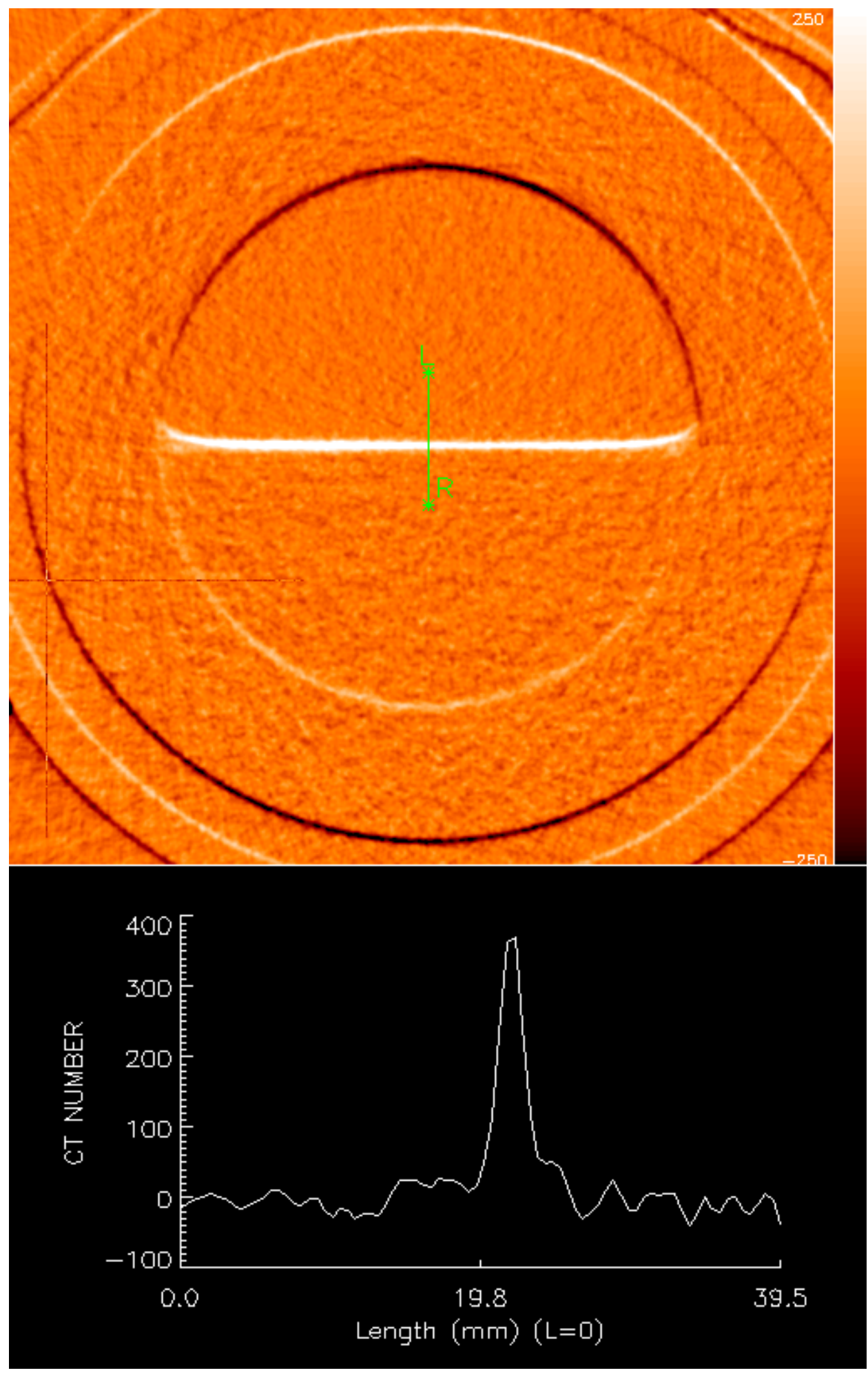


Figure 1.18. Base Scan Subtracted from Fourth Scan White Layer Seen in the Image with $L-R$ Marking is the Hydrate Layer
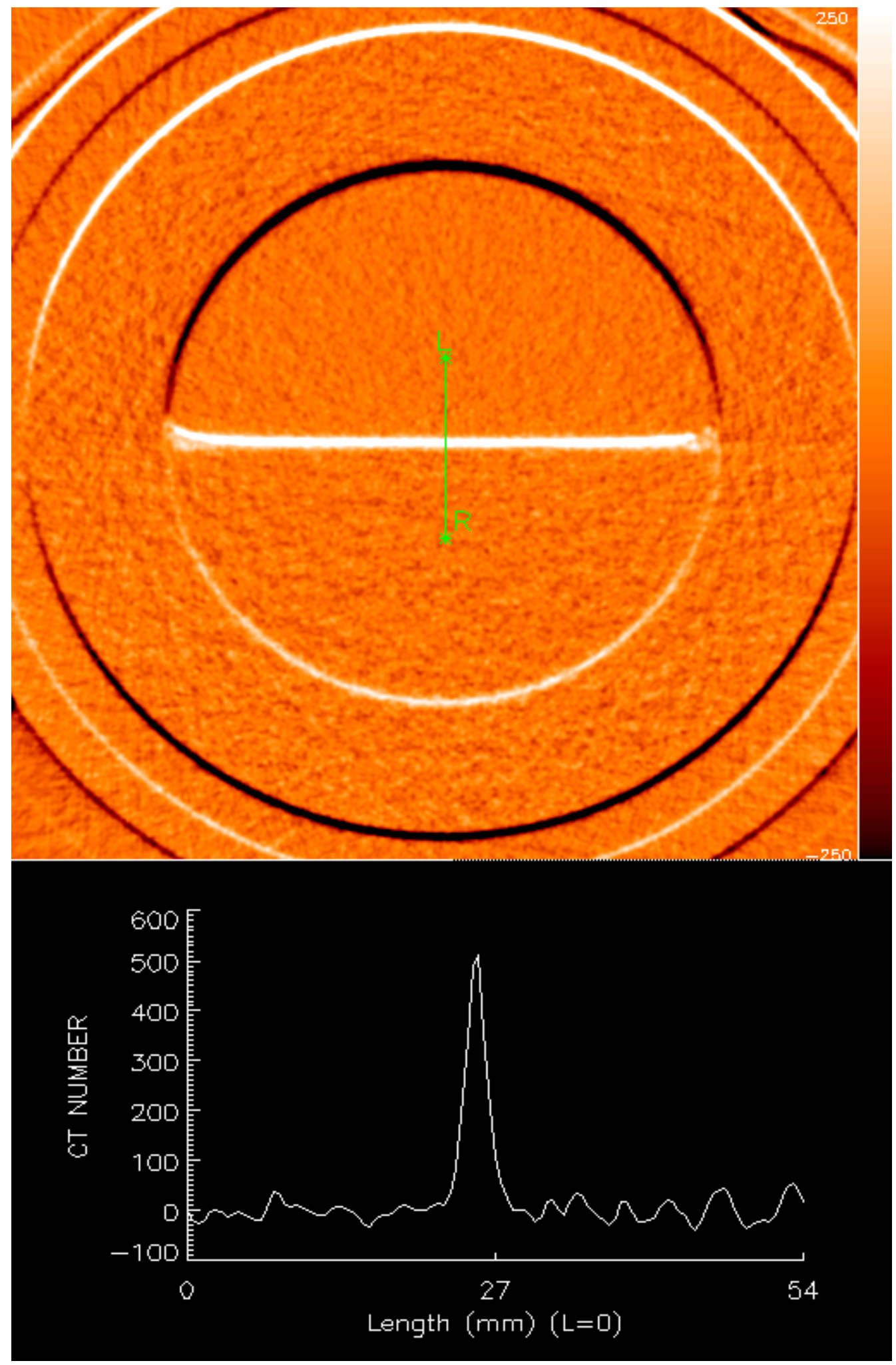


\subsection{NUMERICAL SIMULATION METHODS}

The original Statement of Project Objectives specified that a new hydrate reservoir simulator model be developed and validated. During the first Progress Review Meeting in March 2003, NETL directed that no further work be done on the new reservoir simulator, and that the TOUGHFx/Hydrate simulator developed at the Lawrence Berkeley National Laboratory was to be used. The modeling of the experiments was therefore conducted using the TOUGHFX/HYDRATE simulator.

\section{$2.1 \quad$ Modeling Methods}

The TOUGHFX/HYDRATE simulator could model non-isothermal hydrate reaction, phase behavior, flow of fluids and heat in porous media. It had both equilibrium and kinetic options for hydrate formation and dissociation. The model could handle up to four components (methane, water, hydrate and an inhibitor) and four phases (gas, liquid, hydrate and ice). It used a fully-implicit formulation. More details about this simulator can be found elsewhere (Moridis, Kowalsky, and Pruess, 2005). In the depressurization step of the experiments, fluid was withdrawn at a constant volumetric flow rate. The simulator offered several boundary conditions, but constant volumetric flow rate at a well was not one of them. The pressure was well known at the production well during this depressurization and it varied with time. The simulator had an option of specifying a constant pressure boundary condition. Thus, the pressure decline was discretized into 100 small steps as a function of time. The simulation was conducted for each incremental time step and the final output used for the initialization of the next time step. This procedure is described in detail in the next section. This procedure needed a certain amount of automation for this process. Also, the input and output format for this simulator was not user friendly.

\subsection{Simulator Input Output Processing}

One of the major challenges in running the TOUGHFX/HYDRATE simulator was the complexity of the input and output files. The input and output files were very similar to it predecessor, TOUGH2. The input and output files contained large amount of strings and required advanced string processing algorithms to extract the important data and disregard the unimportant ones. An Excel/VBA code was developed as the Graphical User Interface (GUI) for the input file. The Visual Basic for Application (VBA) code used string processing functions to extract information from the input file, and display it in particular cells in Excel. Changes could be made to the various input values in appropriate cells and the VBA macro used string processing function to make the input file readable by the TOUGHFX/HYDRATE simulator.

In the case of the output file Excel/VBA was not efficient to extract all the information, a combination of a standalone C++ code (TFXRead) and Matlab code (TFXPlot) was used to extract and visualize the data generated by the simulator. A C++ code was developed using GNU C++ in windows using advanced 
string processing. The code would scan through the data files line by line and attempt to match certain key words. If the keyword was found, then the code split the line into appropriate data sets and stored them in a separate text file. The code extracted the following information:

1. Element (grid block) names

2. Respective position of grid blocks

3. Volume of each grid block

4. Connection with other grid blocks

5. Initial Conditions

6. Time

7. For each grid block
a. Pressure
b. Temperature
c. Hydrate Saturation
d. Aqueous Saturation
e. Gas Saturation
f. Ice Saturation

8. For each connection
a. Heat Flux
b. Gas Flux
c. Aqueous Flux
d. $\mathrm{CH} 4$ in Gas Flux
e. $\mathrm{CH} 4$ in Aqueous Flux
f. Gas Velocity
g. Aqueous Velocity

The results for each iteration were stored in a separate text file and numbered consecutively. To plot the data generated from the simulator, MATLAB 7.2 was used. Figure 2.1 shows a 2D plot of the grid blocks. Figure 2.2 shows the temperature distribution throughout the core in a typical simulation. Similar plots can be generated for other variables which the user wishes to view. A copy of the installation and user instruction manual for the hydrates data co-processing software is included in Appendix B. 
Figure 2.1. Plot of a Core ( $L=12$ in., $D=1.5 i n)$ Divided in $R-Z$ Coordinates into $20 \times 20$ Grid Blocks Each Grid Block's Label is also shown

The Color Bar (Blue) is the Core and (Brown) is Boundary

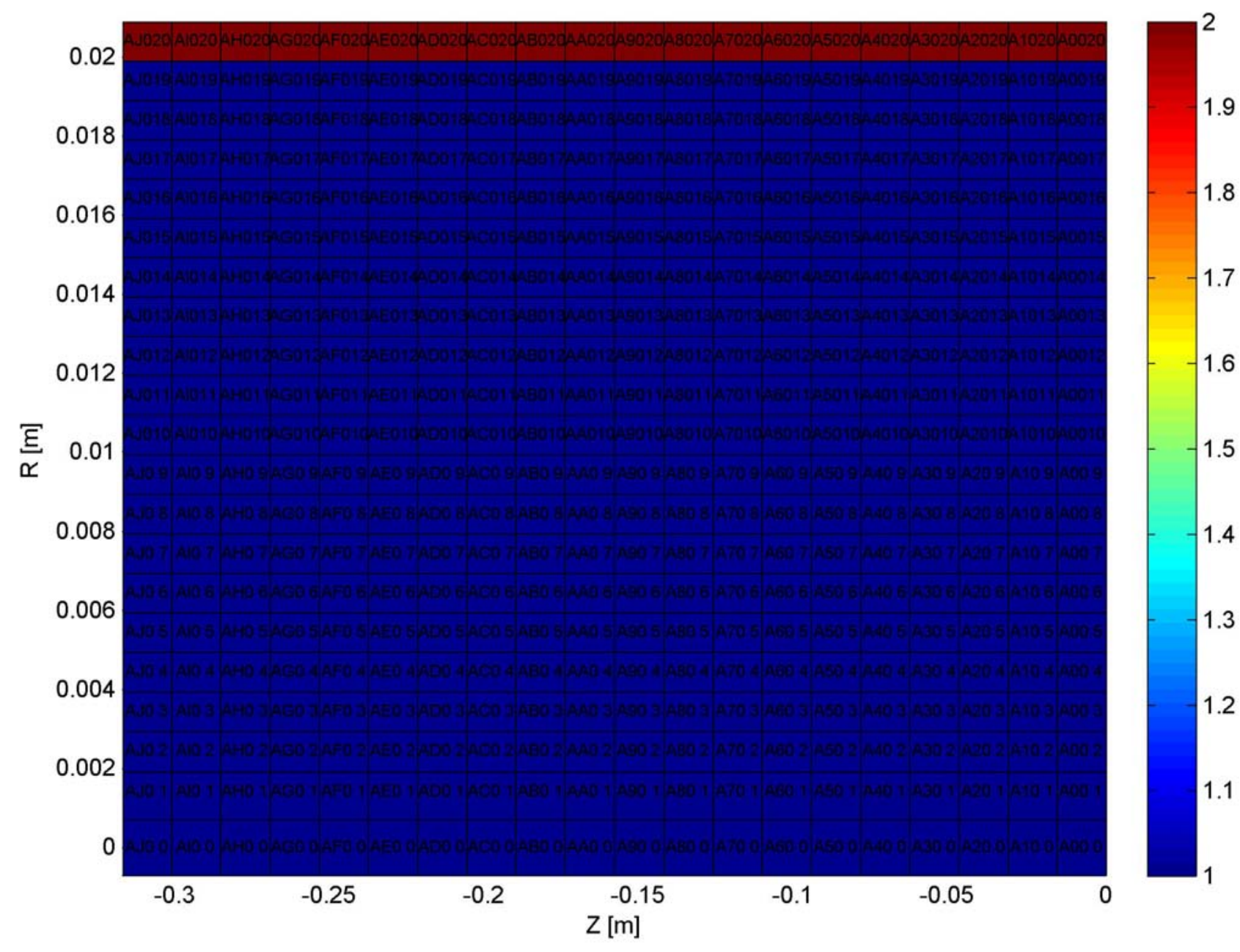


Figure 2.2. Temperature Distribution across the Core

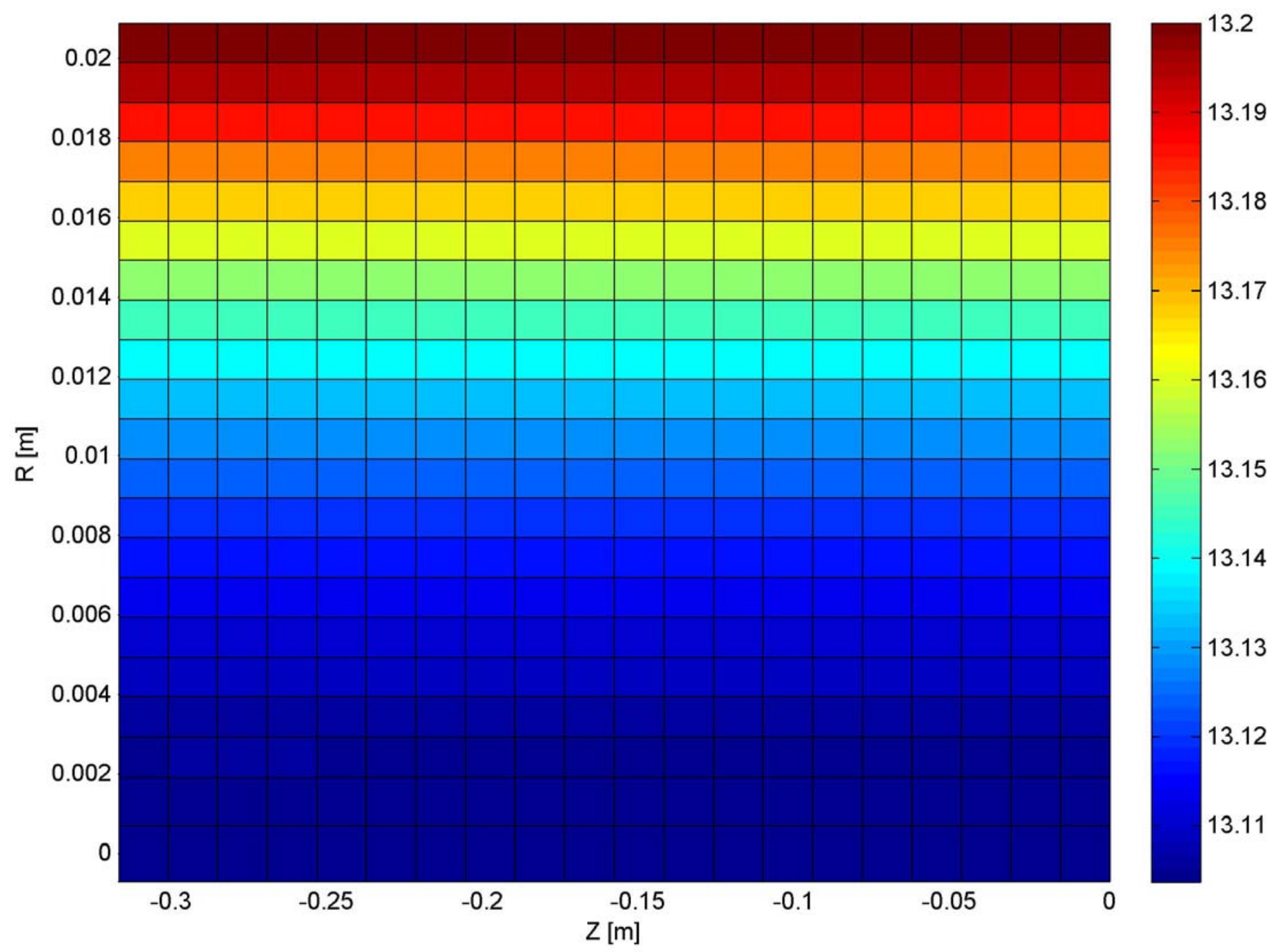




\subsection{RESULTS AND DISCUSSION}

\subsection{Experimental Results}

Using the procedures, equipment and materials described in Section 1, a series of experiments were performed to provide data for hydrate formation and dissociation in porous media with various porosities. A summary of the experiments is shown in Table 3.1.1, following.

Table 3.1.1, Hydrates Test Summary

\begin{tabular}{|c|c|c|c|c|c|c|c|c|}
\hline Test ID & Core Material & $\begin{array}{l}\text { Length } \\
\text { (cm) }\end{array}$ & \begin{tabular}{|c} 
Diameter \\
(cm)
\end{tabular} & $\begin{array}{c}\text { Pore Volume } \\
\text { (cc) }\end{array}$ & $\begin{array}{c}\text { Porosity } \\
(\%)\end{array}$ & $\begin{array}{c}\text { Permability } \\
\text { (md) }\end{array}$ & Hydrate Formation Procedure & Hydrate Dissociation Procedure \\
\hline 1 & None & & & & & & Constant Methane $\mathrm{P}$, Decreasing T & Constant $\mathrm{P}$, Increasing $\mathrm{T}$ \\
\hline 2 & QF50 C & 32.15 & 3.80 & 144.0 & 39.4 & 79000 & Constant Methane $P$, Decreasing $T$ & Constant Erine $P$, Decreasing T \\
\hline 3 & QF50 C & 32.15 & 3.80 & 144.0 & 39.4 & 79000 & Constant Methane $\mathrm{P}$, Decreasing $\mathrm{T}$ & Constant Brine $P$, Decreasing T \\
\hline 4 & QF10N & 24.92 & 3.84 & 101.0 & 35.0 & 29000 & Constant Methane $P$, Decreasing $T$ & Constant Brine $P$, Decreasing T \\
\hline 5 & QF10日 & 30.38 & 3.79 & 133.0 & 38.0 & 29000 & Constant Methane $\mathrm{P}$, Decreasing $\mathrm{T}$ & Pressure Depletion \\
\hline 6 & QF10日 & 30.38 & 3.79 & 133.0 & 38.0 & 29000 & Constant Methane $\mathrm{P}$, Decreasing $\mathrm{T}$ & Constant Brine $P$, Decreasing $T$ \\
\hline 7 & QF10B & 30.38 & 3.79 & 133.0 & 38.0 & 29000 & Constant Methane $P$, Decreasing T & Pressure Depletion \\
\hline 8 & QF10日 & 30.38 & 3.79 & 133.0 & 38.0 & 29000 & Constant Methane $\mathrm{P}$, Decreasing $\mathrm{T}$ & No Dissociation \\
\hline 9 & QF10日 & 3038 & 3.79 & 133.0 & 38.0 & 29000 & Constant Brine P, Decreasing T & No Dissociation \\
\hline 10 & QF10日 & 3038 & 3.79 & 133.0 & 38.0 & 29000 & Constant Brine $P$, Decreasing $T$ & Pressure Depletion \\
\hline 11 & QF10G & 30.16 & 3.79 & 130.0 & 36.0 & 29000 & Constant Erine $P$, Decreasing $T$ & Pressure Depletion \\
\hline 12 & $\mathrm{CiF} 10 \mathrm{G}$ & 30.16 & 3.79 & 130.0 & 36.0 & 29000 & Constant Brine $P$, Decreasing $T$ & Pressure Depletion \\
\hline 13 & Simulated GOM & 29.00 & 3.79 & 123.0 & 46.0 & & Constant Brine $P$, Decreasing $T$ & Pressure Depletion \\
\hline
\end{tabular}

A base run was performed to obtain base line data for hydrate formation and dissociation in the absence of porous media. Synthetic core were placed in a CT scannable core holder. At a temperature and pressure outside the hydrate formation region, a fixed ratio of brine/gas was injected to saturate the core to the target water/gas saturation. Hydrate formation was induced by lowering the temperature to the conditions conducive to hydrate formation.

Two different experimental approaches were used to achieve these conditions. The first approach (constant methane pressure) was to lower the temperature while the system pressure was kept constant by maintaining a constant gas pressure. During the experiment, the gas consumption was quantified. The second approach (constant methane mass) was to lower the system temperature with a constant mass of methane while maintaining the system pressure with brine.

Following are the results from the individual tests. 


\subsubsection{Initial Gas Hydrate Generation Test, No Core Material (Test 1)}

Core Material:

Permeability (md):

Hydrate Formation Procedure:

Hydrate Dissociation Procedure:
None

Constant methane $\mathrm{P}$, decreasing $\mathrm{T}$

Constant $P$, increasing $T$

The purpose of this test was to obtain base line data for hydrate formation and dissociation in the absence of porous media. For gas hydrate formation in a bulk system, 6-wt\% sodium iodide solution was used as brine. Oil was used as the overburden fluid. The net confining stress (pore pressure minus gross overburden pressure) on the boot was maintained close to 0 psi during the entire experiment. The entire experiment was conducted with the core holder in a horizontal position on the CT gantry table. The boot was initially filled up to $75 \%$ with brine solution. The remaining $25 \%$ of the boot was filled with methane gas. The brine solution, methane gas and overburden fluid pressure were slowly ramped to 2,500 psig. The entire system was maintained at $70^{\circ} \mathrm{F}$ during pressurization of the fluids.

Methane gas was bubbled at $1.0 \mathrm{cc} / \mathrm{min}$ at $2,500 \mathrm{psig}$ through a gas-bubbling tube located near the bottom of the boot while overburden fluid temperature was maintained at $70^{\circ} \mathrm{F}$. Methane gas flow was continued through brine solution for at least 6-hours at $70^{\circ} \mathrm{F}$. Gas flow was then stopped and CT scan and acoustic scans were performed. At 2,500 psig and $70^{\circ} \mathrm{F}$, hydrate formation was not detected either by CT scan or by acoustic method. Methane gas flow through brine solution was again started at $70^{\circ} \mathrm{F}$. Overburden fluid temperature was cooled to $65^{\circ} \mathrm{F}$ in 1-hour. Methane gas flow was continued at 1 $\mathrm{cc} /$ hour for an additional 6-hours. CT scan and acoustic scans were performed again at $65^{\circ} \mathrm{F}$ and 2,500 psig. Again, hydrate formation inside the boot could not be detected at $65^{\circ} \mathrm{F}$. The process of $5^{\circ} \mathrm{F}$ per hour cooling, additional 6 hours of gas flow, CT scan and acoustic scans were repeated for temperatures down to $45^{\circ} \mathrm{F}$. Hydrate formation inside the boot could not be detected even after the temperature reached $45^{\circ} \mathrm{F}$ at 2,500 psig.

During cooling of the system to $40^{\circ} \mathrm{F}$ from $45^{\circ} \mathrm{F}$, both acoustic scans and CT scan detected formation of hydrate inside the boot. Acoustic measurements indicated hydrate formation inside the boot when overburden fluid temperature ranged between $42.5^{\circ} \mathrm{F}$ and $41.3^{\circ} \mathrm{F}$. Since acoustic scans were performed at 15-minute interval, the exact temperature when hydrate first formed inside the boot could not be determined. Acoustic measurements indicating hydrate formation are shown in Table 3.1.2 and Figure 3.1.1. Acoustic velocity dropped from $1552.7 \mathrm{~m} / \mathrm{sec}$ at $42.5^{\circ} \mathrm{F}$ to $1541.7 \mathrm{~m} / \mathrm{sec}$ at $40.3^{\circ} \mathrm{F}$. Once the temperature reached $40.3^{\circ} \mathrm{F}$, acoustic signal disappeared completely. Acoustic signal came back only after disassociation of hydrate and injection of brine in the boot, indicating that the acoustic transducers were functioning during the course of the experiment. 
The overburden fluid temperature, methane gas injection pressure, pressure at outlet end of core holder, and overburden fluid pressure were monitored during the course of the experiment. These results are shown in Figure 3.1.2. Right after the disappearance of the acoustic signal, all of the measured pressures of the system started to rise indicating higher resistance to gas flow from the gas-bubbling tube to the outlet of the core holder. After the system temperature had reached $39^{\circ} \mathrm{F}$, methane gas flow was stopped but system outlet valve after backpressure regulator was kept open. All the measured pressures came down to pre-hydrate formation level after gas flow was stopped. The system temperature was gradually increased and hydrate dissociation was first detected at $59.3^{\circ} \mathrm{F}$. Gas produced during dissociation was fed in water filled piston cylinder that was maintained at constant pressure through a backpressure regulator. Water was first produced from the piston cylinder after overburden fluid temperature reached $59.3^{\circ} \mathrm{F}$. Figure 3.1 .3 shows the CT images of slices along the length of the core before and after hydrate formation. The white color inside the boot is equivalent to a CT number of $\sim 1050$ and indicates 6 -wt $\%$ Nal solution. The red color at the top of boot is equivalent to a CT number of $\sim-750$ and indicates methane gas. The salt in the brine does not go into the hydrate phase and did not contribute to the CT number. 
Table 3.1.2. Measured Acoustic Velocity as a Function of Temperature during Hydrate Formation in Bulk Sodium lodide (Nal) Solution

\begin{tabular}{|c|c|c||c|c|c||}
\hline Date and Time & $\begin{array}{c}\text { Overburden } \\
\text { Fluid T, }\left({ }^{\circ} \mathrm{F}\right)\end{array}$ & $\begin{array}{c}\text { Acoustic } \\
\text { Velocity, } \\
(\mathrm{m} / \mathrm{sec})\end{array}$ & Date and Time & $\begin{array}{c}\text { Overburden Fluid } \\
\mathrm{T},\left({ }^{\circ} \mathrm{F}\right)\end{array}$ & $\begin{array}{c}\text { Acoustic } \\
\text { Velocity, } \\
(\mathrm{m} / \mathrm{sec})\end{array}$ \\
\hline $8 / 1 / 037: 08$ & 45.4 & 1553.517 & $8 / 1 / 0310: 55$ & 45.3 & 1552.963 \\
\hline $8 / 1 / 037: 23$ & 45.5 & 1553.121 & $8 / 1 / 0311: 11$ & 45.5 & 1553.121 \\
\hline $8 / 1 / 037: 39$ & 45.8 & 1553.121 & $8 / 1 / 0311: 26$ & 45.5 & 1552.884 \\
\hline $8 / 1 / 037: 54$ & 45.7 & 1552.725 & $8 / 1 / 0311: 41$ & 45.5 & 1552.725 \\
\hline $8 / 1 / 038: 09$ & 45.7 & 1552.725 & $8 / 1 / 0311: 56$ & 44.8 & 1552.567 \\
\hline $8 / 1 / 038: 24$ & 45.6 & 1552.725 & $8 / 1 / 0312: 12$ & 43.8 & 1552.725 \\
\hline $8 / 1 / 038: 39$ & 45.6 & 1552.725 & $8 / 1 / 0312: 27$ & 42.5 & 1552.725 \\
\hline $8 / 1 / 038: 54$ & 45.8 & 1552.963 & $8 / 1 / 0312: 42$ & 41.3 & 1551.382 \\
\hline $8 / 1 / 039: 09$ & 45.6 & 1552.725 & $8 / 1 / 0312: 57$ & 40.9 & 1549.568 \\
\hline $8 / 1 / 039: 25$ & 45.6 & 1552.963 & $8 / 1 / 0313: 12$ & 40.6 & 1547.208 \\
\hline $8 / 1 / 039: 40$ & 45.7 & 1552.963 & $8 / 1 / 0313: 28$ & 40.5 & 1545.247 \\
\hline $8 / 1 / 039: 55$ & 45.7 & 1552.725 & $8 / 1 / 0313: 43$ & 40.5 & 1543.682 \\
\hline $8 / 1 / 0310: 10$ & 45.5 & 1552.725 & $8 / 1 / 0313: 58$ & 40.3 & 1541.73 \\
\hline $8 / 1 / 0310: 25$ & 45.5 & 1553.121 & & & \\
\hline $8 / 1 / 0310: 40$ & 45.4 & 1552.963 & & & \\
\hline \hline
\end{tabular}


Figure 3.1.1. Test 1: Measured Acoustic Velocity as a Function of Temperature during Hydrate Formation in Bulk Sodium lodide (Nal) Solution

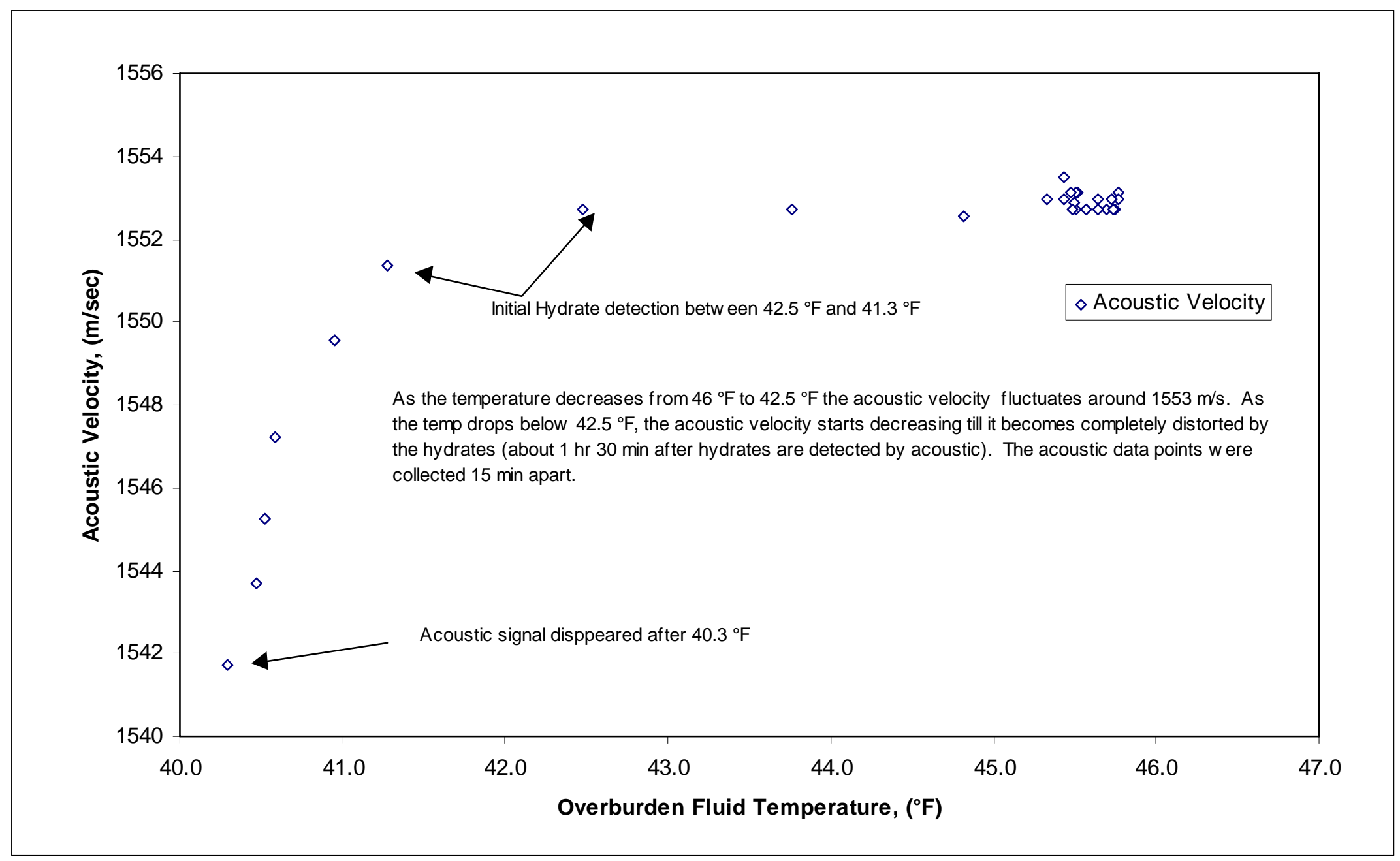


Figure 3.1.2. Test 1: Flow Data during Hydrate Formation Test with Bulk Sodium lodide Solution

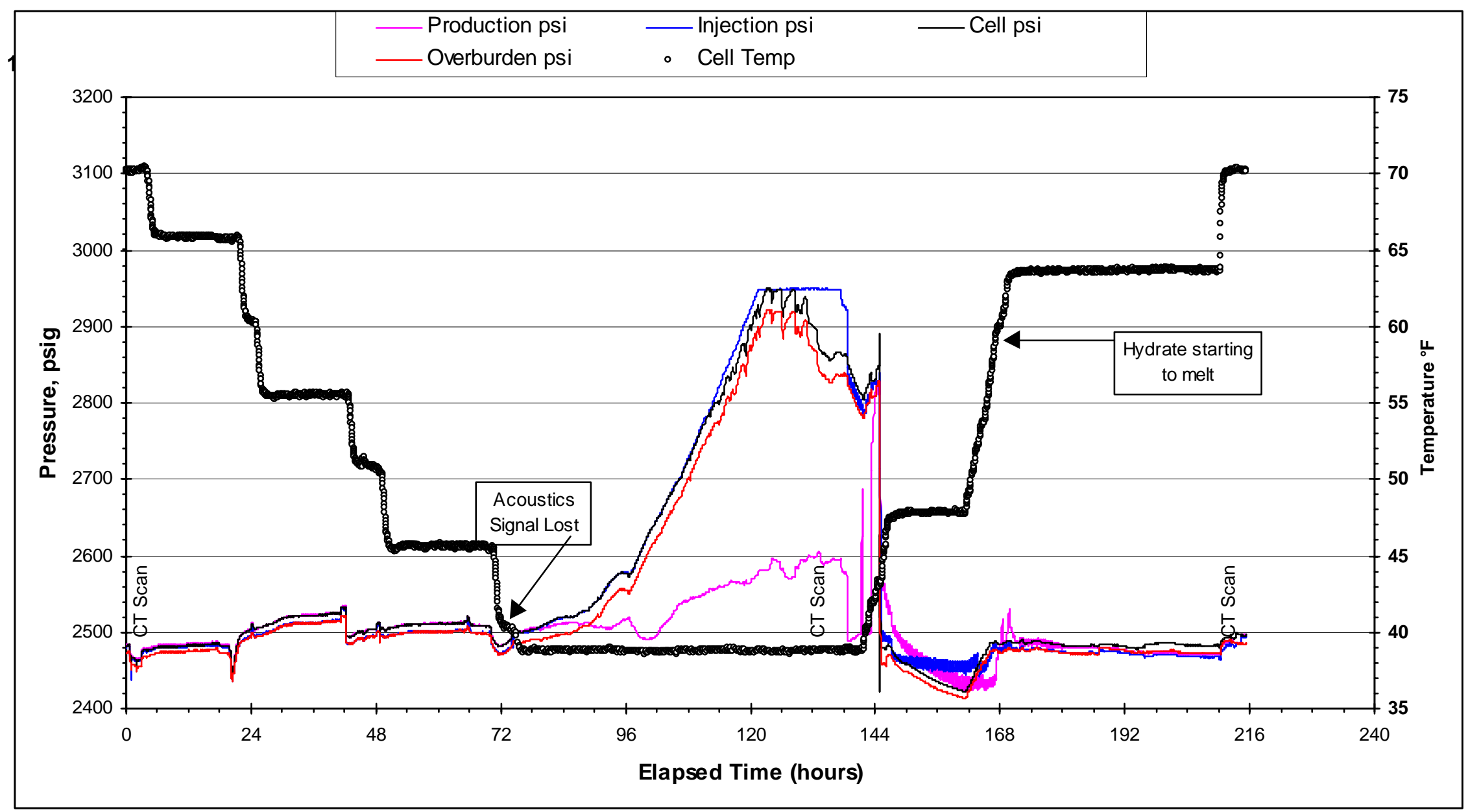


Figure 3.1.3. Test 1: CT Scan Images before and after Hydrate Formation

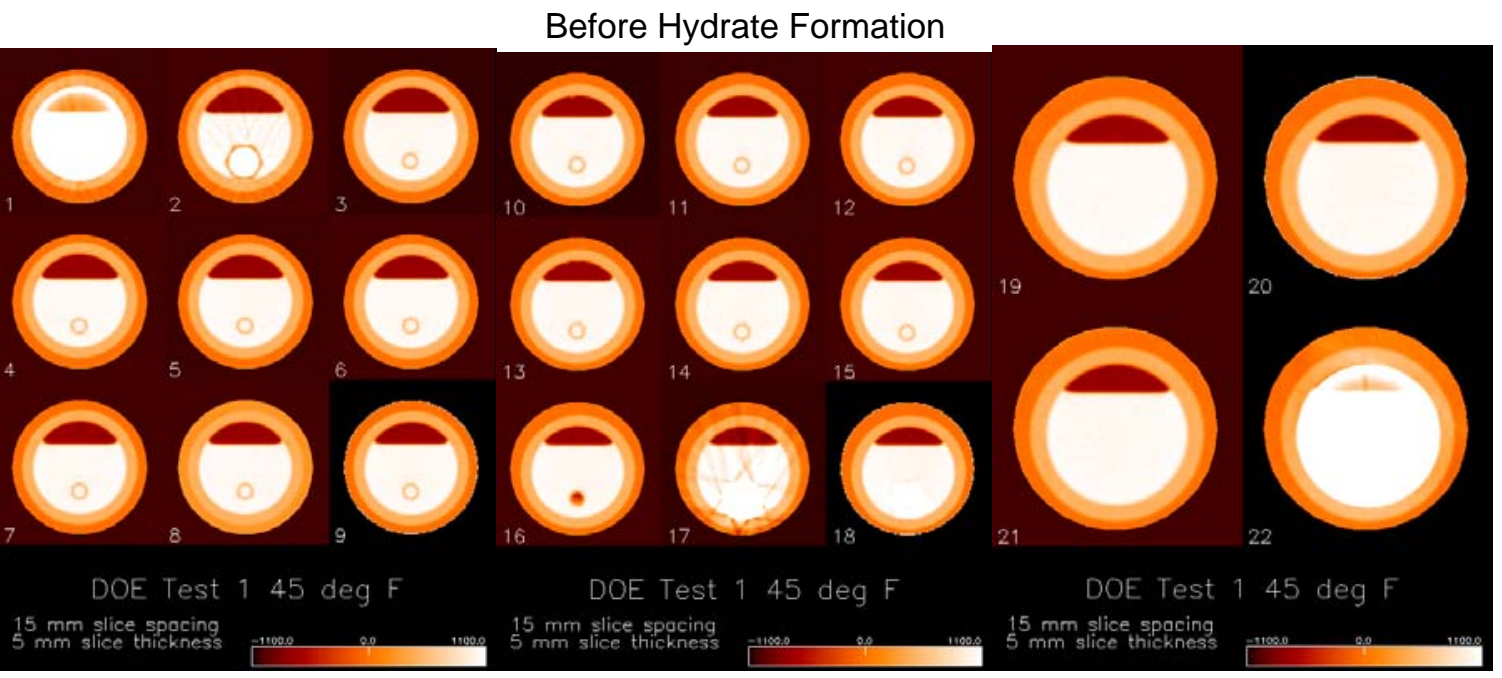

After Hydrate Formation

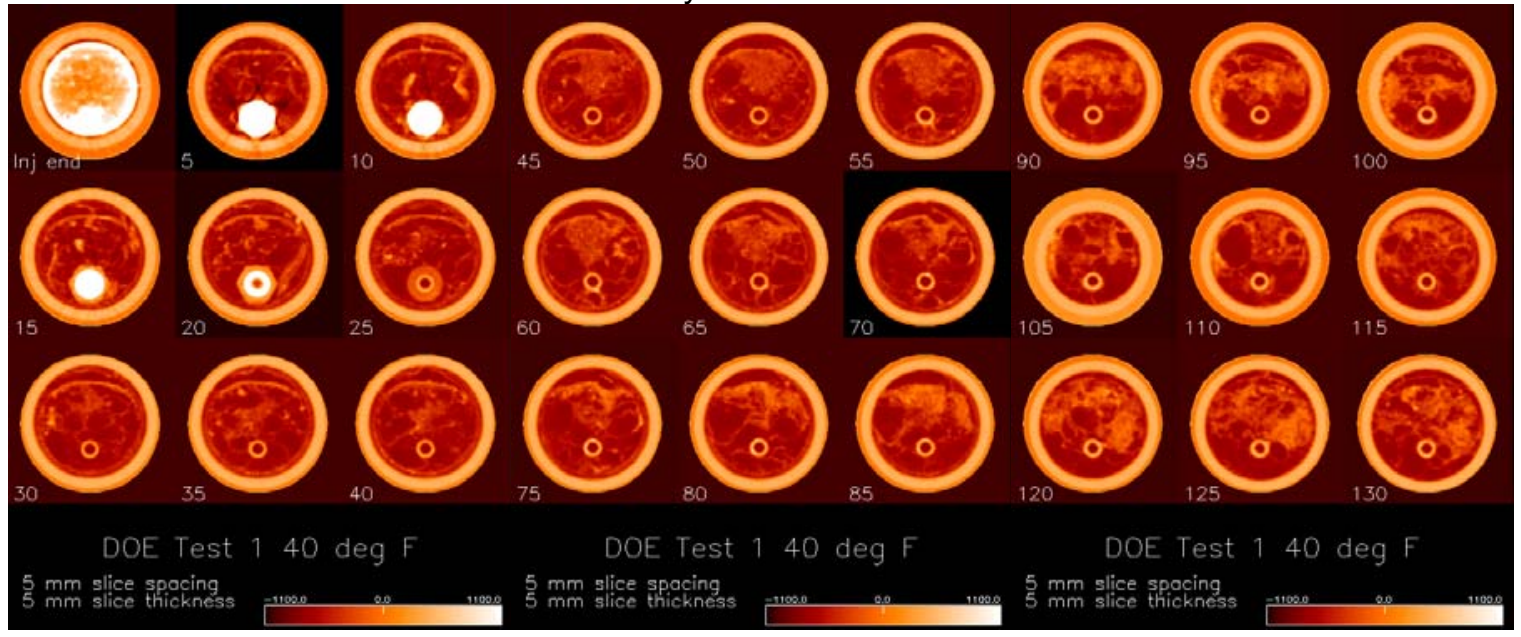




\subsubsection{Hydrate Formation in High Permeability Core (Test 2)}

Core Material:

Permeability (md):

Hydrate Formation Procedure:

Hydrate Dissociation Procedure:
QF50C

79,000

Constant methane $\mathrm{P}$, decreasing $\mathrm{T}$

Constant $P$, increasing $T$

This was one of the initial tests of the equipment and materials in the presence of a core sample. QF-50 was used for formation and dissociation of hydrates. Gas permeability of this porous medium was previously measured as 77-78 Darcy.

A 12" long, 1.5" diameter core of QF-50 was encased in a rubber boot and boot was mounted in a CT scannable core holder. The core was solvent cleaned, dried, CT scanned, and acoustic velocity measured across the core. The core was then saturated with 6 -wt\% sodium iodide (Nal) solution, CT scanned, and acoustic velocity measured. Measured and calculated porosity of the core from material balance and CT, respectively, showed close agreement. The pore volume of the porous medium was $131 \mathrm{cc}$. Out of $131 \mathrm{cc}, 63 \mathrm{cc}$ of Nal water was drained from the core leaving $68 \mathrm{cc}$ of water in the core (3.78 moles of water). During water draining, top of core was connected with a methane supply tank. The core was CT scanned and acoustic velocity in the core was measured.

The experiment was conducted with core lying in horizontal mode. During injection mode, pump injected fluid at top-diameter of core from both faces. During withdrawal mode, pump withdrew fluid at bottomdiameter of core.

The bath temperature was cooled to $48^{\circ} \mathrm{F}$ from $70^{\circ} \mathrm{F}$ over 24 -hour period at 2,500 psia. Core temperature was $50^{\circ} \mathrm{F}$ when bath temperature stabilized at $48^{\circ} \mathrm{F}$. For the next 5-days, bath temperature was maintained at $48^{\circ} \mathrm{F}$ and core was repeatedly CT scanned. All along, acoustic velocity data was recorded at 20-minutes interval. During the initial 2-days, no changes in the core were detected. On the $3^{\text {rd }}$ day, methane injection in the core by the pump increased dramatically indicating hydrate formation. After 5-days, bath temperature was cooled to $44^{\circ} \mathrm{F}$ and core temperature reached $45^{\circ} \mathrm{F}$. Cooling of the core to $45^{\circ} \mathrm{F}$ from $50^{\circ} \mathrm{F}$ did not affect methane consumption rate. Bath temperature was gradually heated to $58^{\circ} \mathrm{F}$ over 6 -days. During heating, methane consumption rate stayed steady at $1.5 \mathrm{cc}$ per day. During heating, as temperature started to rise, fluid was withdrawn by pump from the core to compensate for increase in volume. The withdrawn fluid was always passed through an online densitometer. During heating of the core to $58^{\circ} \mathrm{F}$, density of the withdrawn fluid corresponded to methane density. Absence of water in the withdrawn fluid indicated absence of mobile water phase in the core.

When bath temperature was raised to $61^{\circ} \mathrm{F}$ (core temperature $60^{\circ} \mathrm{F}$ ) from $58^{\circ} \mathrm{F}$, hydrates in the core started dissociating and pump started withdrawing fluid from bottom-diameter at a high rate. Density of 
withdrawn fluid corresponded to density of water. During hydrate formation phase, water was absent from occasional fluid withdrawn and thus confirming hydrate dissociation. P-wave acoustic velocity also decreased when hydrate dissociation was initiated. Entire hydrate dissociated in first 24-hours and no additional fluid was withdrawn during next 72-hours. During dissociation, 20 cc of methane was withdrawn at 2,500 psia and $61^{\circ} \mathrm{F}$. Based on methane density of $0.1402 \mathrm{gm} / \mathrm{cc}$ at 2,500 psia and $61^{\circ} \mathrm{F}$, 0.1748 mole of methane was withdrawn from the core during dissociation. Based on initial 3.78 moles of water present in the core, 0.0463 mole of methane per mole of available water in the core was produced during dissociation.

During hydrate formation phase, $33 \mathrm{cc}$ of methane was injected by pump at 2,500 psia and $70^{\circ} \mathrm{F}$. $63-\mathrm{cc}$ of methane was present in the core at 2,500 psia and $50^{\circ} \mathrm{F}$ prior to hydrate formation. In total, 0.853 mole of methane was introduced in the core. During dissociation, withdrawn fluid was always water indicating $\sim 83 \mathrm{cc}$ of pore space in core was occupied by methane gas at 2,500 psia and $61^{\circ} \mathrm{F}$ corresponding to 0.725 mole of methane. 0.127 mole of methane gas is unaccounted for.

During hydrate formation stage, there was a constant rate of methane gas consumption even after 10days. The initial presumption was the presence of a minor leak in the system. However, during dissociation phase, a constant pump volume reading indicated absence of leaks in the system. For overburden fluid, 3 liter of mineral oil instead of water was used to protect against short-circuiting of acoustic connections.

The data obtained in Test 2 are presented in Figures 3.1.4 and 3.1.5, following. Figures 3.1.6 through 3.1.8 show CT scan images obtained at various times during the test. The color scale at the bottom of the figures indicate the relative CT number and color depicted in the image. Higher CT numbers correspond to areas of increased densities in the image. The image slices were taken from left to right, starting from the methane supply side of the core holder. Subtracted CT images (Figure 3.1.9) indicated appearance of highly x-ray attenuating material in the core. The high CT numbers are presumed to occur because as hydrates are formed, $\mathrm{Nal}$ is expelled from hydrate cage, causing free-water phase to become concentrated in Nal, a high x-ray attenuating material.

The resistivity electrodes were not functional. Consequently, no resistivity data were available. 
Figure 3.1.4. Test 2: Hydrate Formation at Constant Methane Pressure

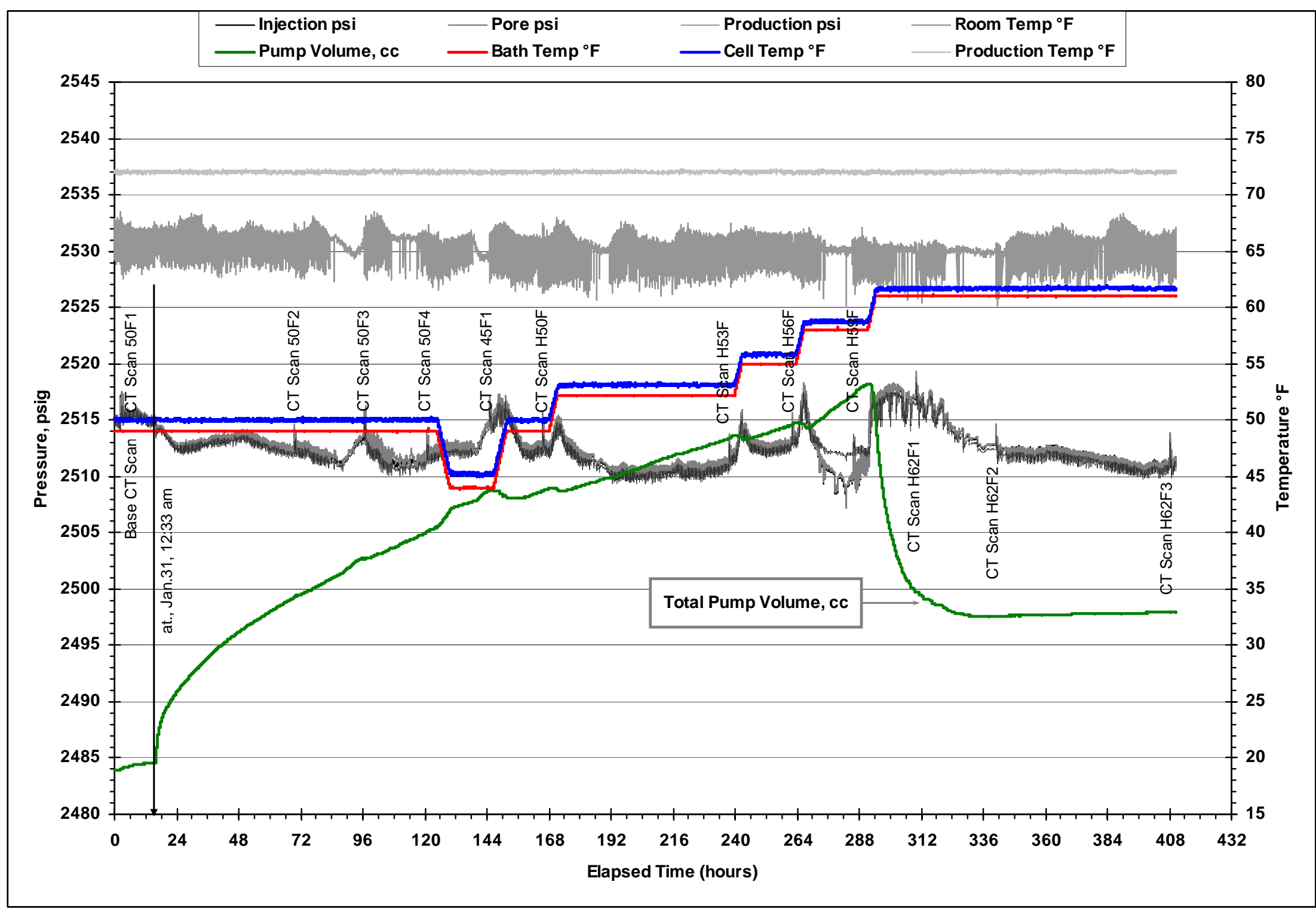


Figure 3.1.5. Test 2: Acoustic Velocity during Hydrate Formation and Dissociation

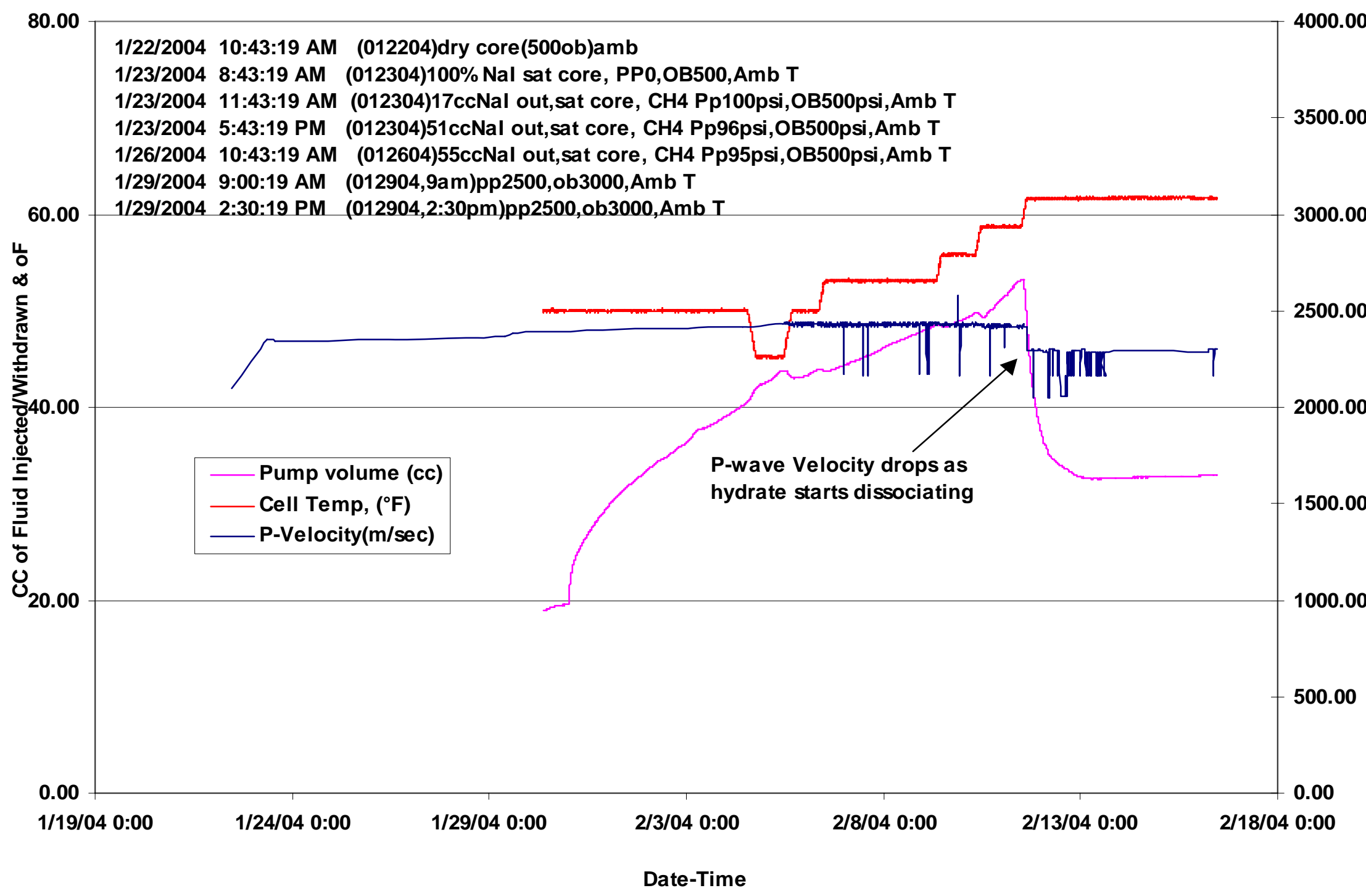


Figure 3.1.6. Test 2: CT Images of Core after Draining 63-cc of Water from Core (Red color in this image indicates primarily gas saturated, whereas white areas indicate primarily brine saturated zones)
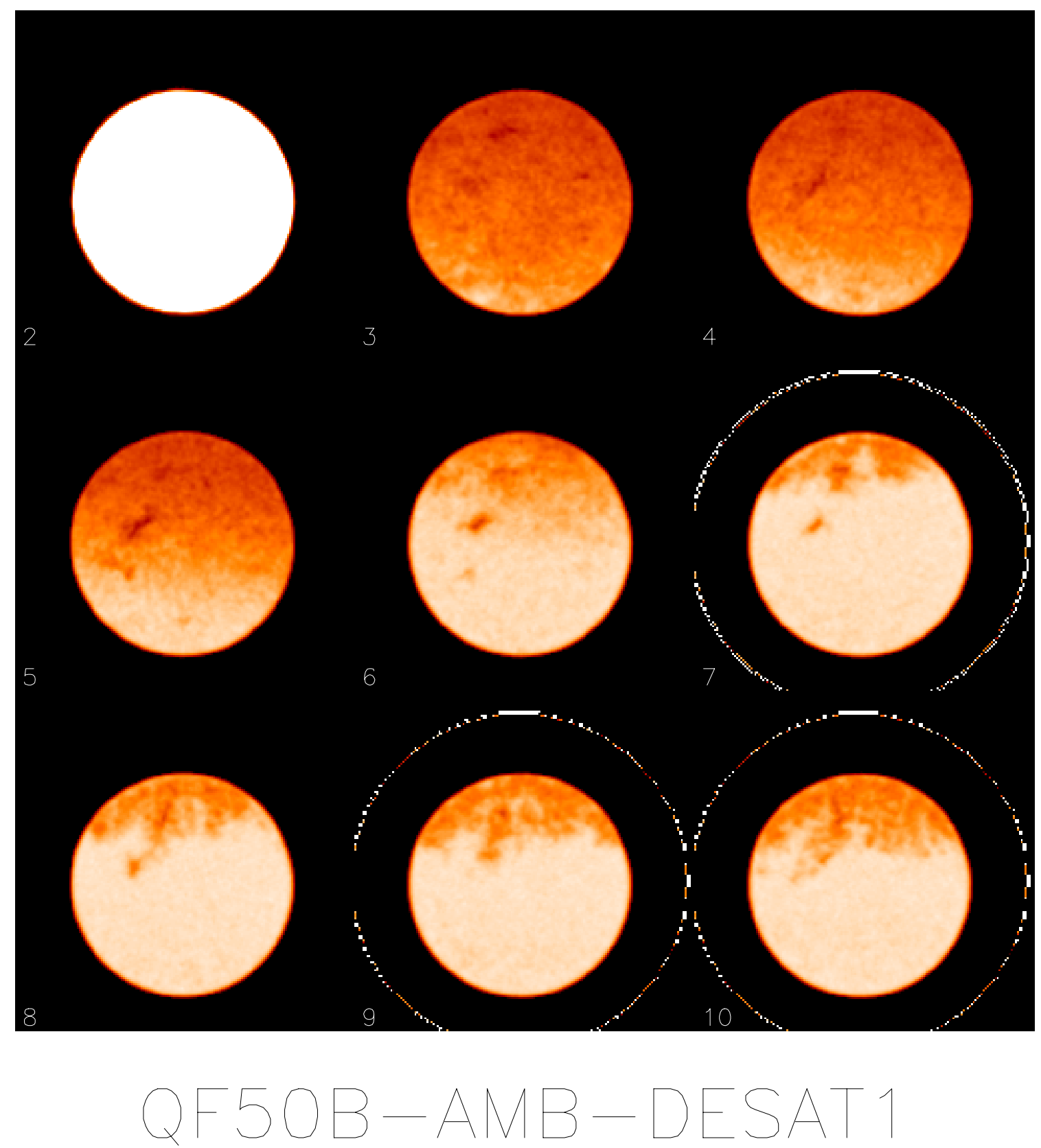

$393 \mathrm{~mm}$ slice spacing

$2 \mathrm{~mm}$ slice thickness 
Figure 3.1.7. Test 2: CT Image after Hydrate Formation

(After hydrate formation, distinction between gas and water saturated zones disappeared, indicating expansion of liquid/hydrate into previously gas filled zones)
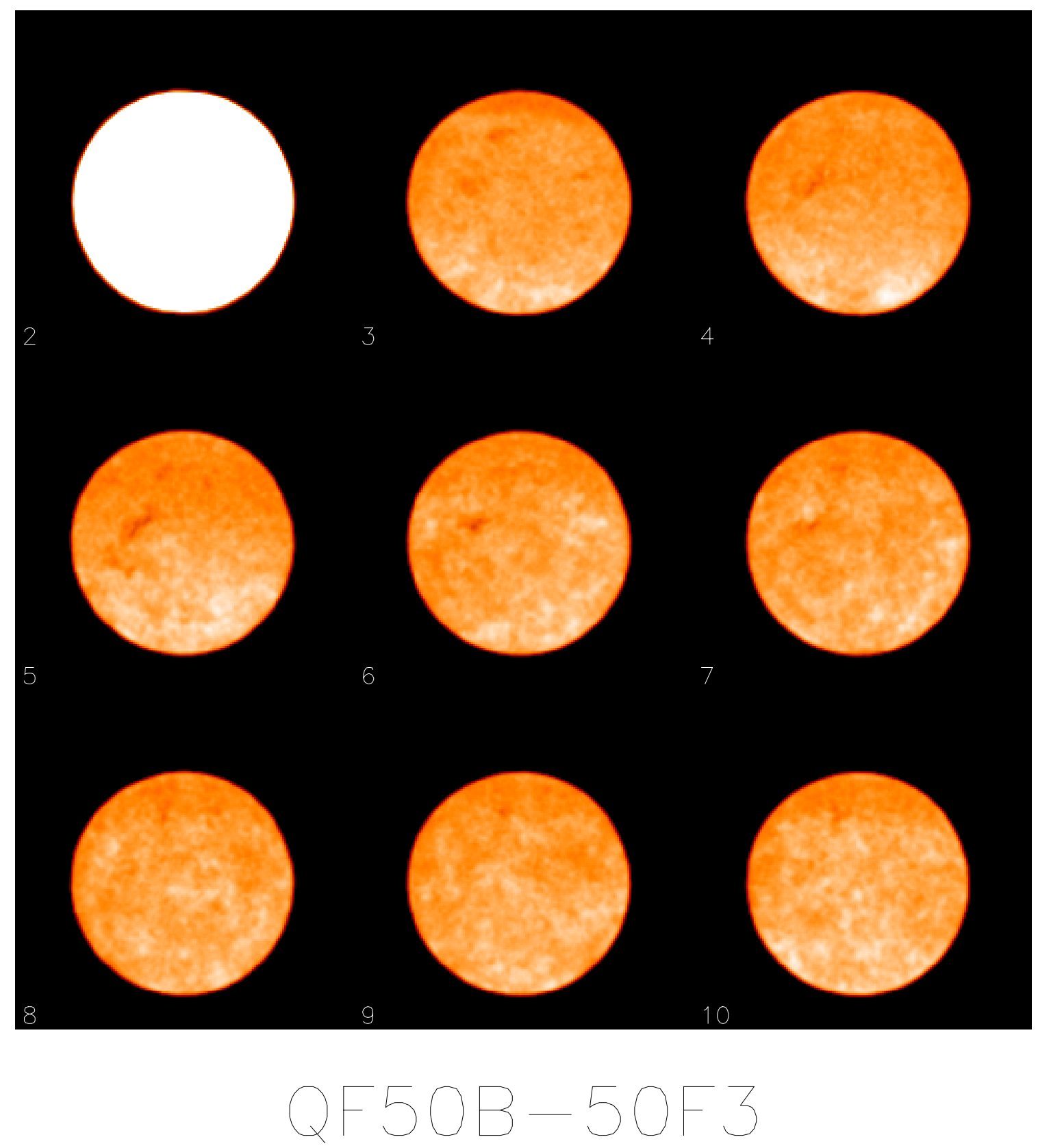

$393 \mathrm{~mm}$ slice spacing

$2 \mathrm{~mm}$ slice thickness

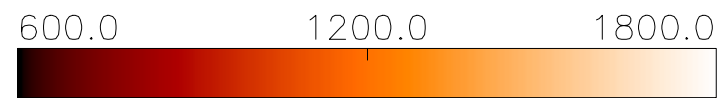


Figure 3.1.8. Test 2: CT Image after Hydrate Dissociation (Note color and texture changes compared to Figure 3.1.7.)
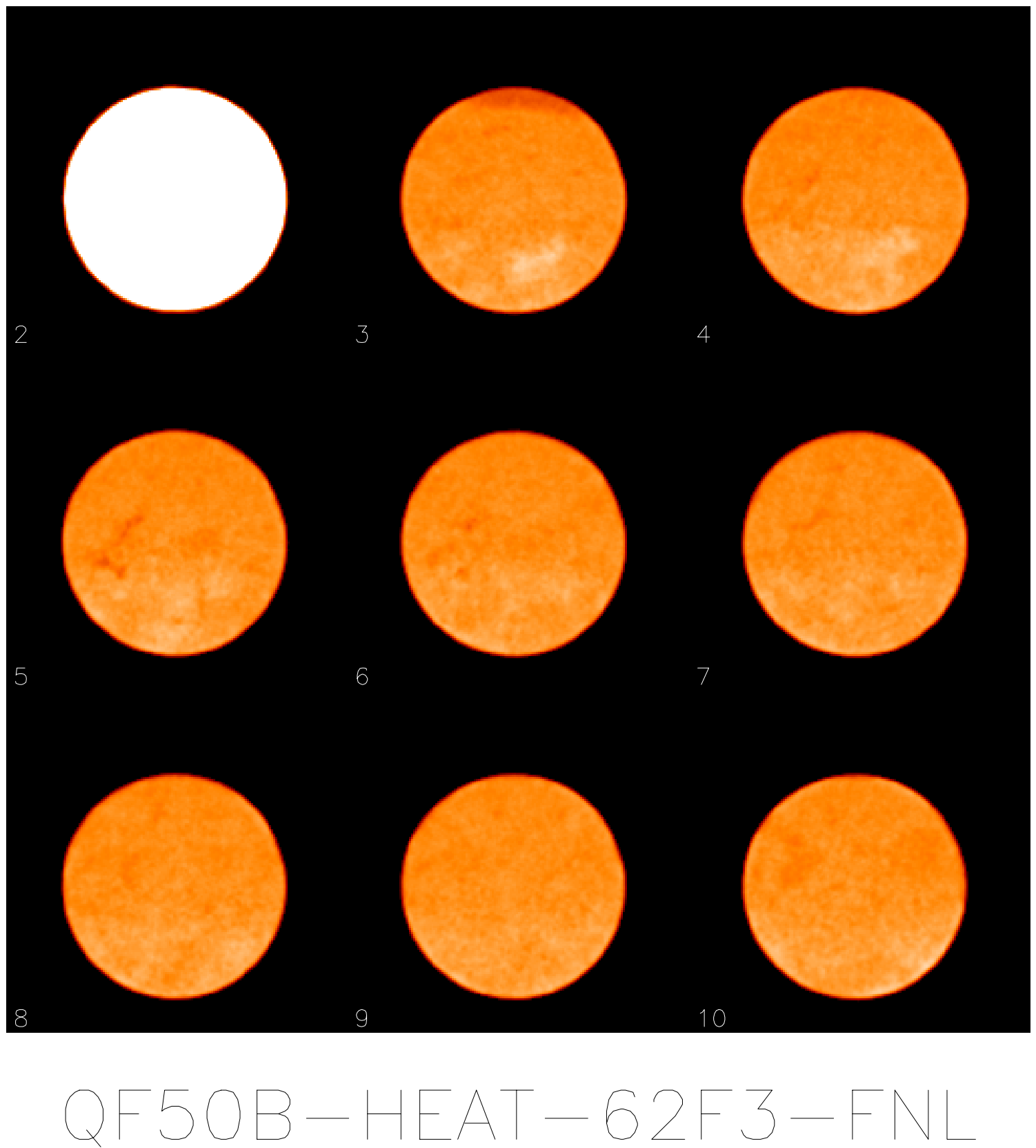

$393 \mathrm{~mm}$ slice spacing

$2 \mathrm{~mm}$ slice thickness 
Figure 3.1.9. Test 2: Subtracted CT Image, CT Images Before Hydrate Formation - CT Images After Hydrate Formation. (white areas or positive numbers indicate that CT numbers decreased substantially, which would occur with hydrate formation. Black or negative numbers indicate that CT numbers increased after hydrate formation. In previously gas occupied zones, CT numbers would increase by invasion of liquid or hydrate).
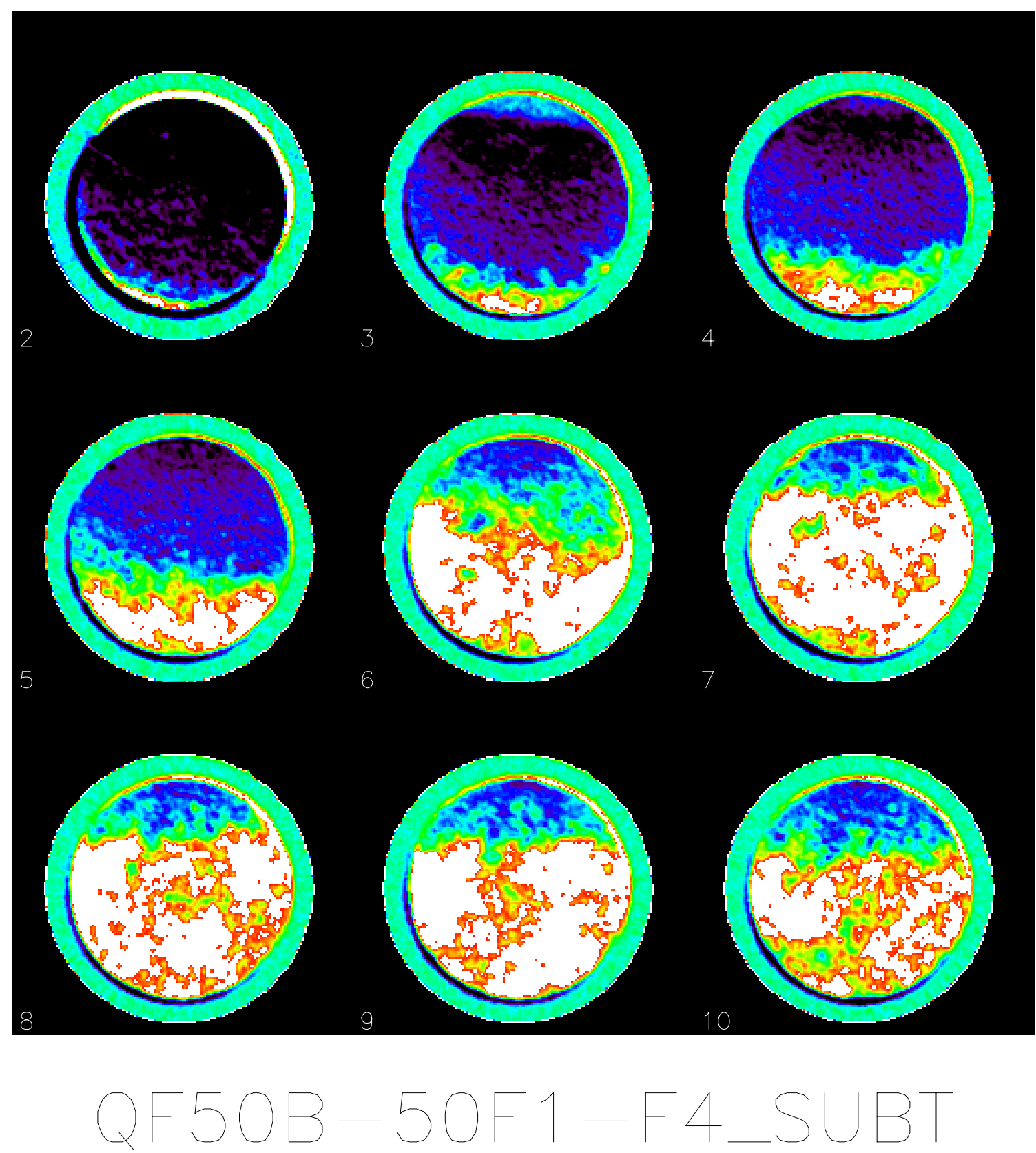

$393 \mathrm{~mm}$ slice spacing

$2 \mathrm{~mm}$ slice thickness

$-250.0$

0.0

250.0 


\subsubsection{Hydrate Formation in High Permeability Core (Test 3)}

Core Material:

Permeability (md):

Hydrate Formation Procedure:

Hydrate Dissociation Procedure:
QF50C

79,000

Constant methane $P$, decreasing $T$

Constant $P$, increasing $T$

Test 3 was a first repeat of Test 2. During Test 3, the boot leaked during the process of bringing the setup to pressure. The experiment was suspended and the fitting on the boot was repaired. The setup was again put together using the QF-50 core, saturated with 6-wt\% Nal, CT-Scanned, de-saturated to $\sim 50 \%$, and CT-Scanned. The setup was brought to test pressure conditions for overburden and pore pressure at room temperature, the setup was then left to equilibrate overnight. During the course of pressure equilibrium, the setup held the pressure for about $8 \mathrm{hrs}$, and then an overburden leak was detected in the jacket.

The leak was monitored for its severity and its effect on the formation of hydrate in the core, and a decision was made to continue the test because the CT scan and resistance data showed that the leak was located near the middle of the jacket. The leak affected only one half of the core, towards the production end of the core, and the pump data indicated that the leak was very small, in the neighbor hood of $\sim 1 \mathrm{cc} / \mathrm{hr}$. The acoustic signal began to deteriorate after the leak. The signal eventually died out completely as the test progressed.

The temperature data was collected on the core using the RTD temperature sensors, embedded in the jacket. During the hydrate formation step, as the cell was cooled at a rate of $2^{\circ} \mathrm{F} / \mathrm{hr}$., the embedded RTD sensor tracked core temperature at an offset of $\sim 2^{\circ} \mathrm{F}$ from the cell temperature. As the hydrate started to form, the cell temperature remained constant but the core temperature showed a sudden peak, indicating the exothermic nature of the hydrate formation. The cell was held at that temperature overnight and monitored till no evidence of further hydrate formation was detected. The hydrate cell was then heated at the rate of $2^{\circ} \mathrm{F} / \mathrm{hr}$. During the heating cycle, core temperature increased at a constant rate till the hydrates started dissociating. At hydrate dissociation temperature, the core temperature dipped indicating endothermic nature of the hydrate dissociation.

Resistance across the core was also measured during the hydrate formation/dissociation test. As the temperate was lowered, the electrical resistance of the core increased linearly. Formation of hydrates in the core caused a steep decline in core resistance that tracked closely to the peak in core temperature profile (Figure 3.1.10). A similar change in core electrical resistance was observed during the hydrate dissociation phase. 
CT-Scan and flow/pressure data on the core was also collected during the course of the experiment. The core was CT-Scanned before cooling ramp (Figure 3.1.12), after hydrate formation (Figure 3.1.13), and after hydrate dissociation (Figure 3.1.14). The CT-Scan images showed marked differences before and after hydrate formation and dissociations. However, the pressure/volume data collected during the test was compromised because of an overburden fluid leak into the core. An improved jacket for the core was designed to eliminate leakage.

No acoustic data was available during this experiment. To determine the cause for loss of the acoustic data during the course of the experiment, various bench-top experiments with acoustic sensors were carried out to isolate the acoustic problem. Various improvements in the acoustic sensor assembly were carried out: 1) redesigning of the wire connection to reduce noise, and 2) use of a small lead piece between the core and the flow distributor. 
Figure 3.1.10. Test 3: Hydrate Formation at Constant Methane Pressure

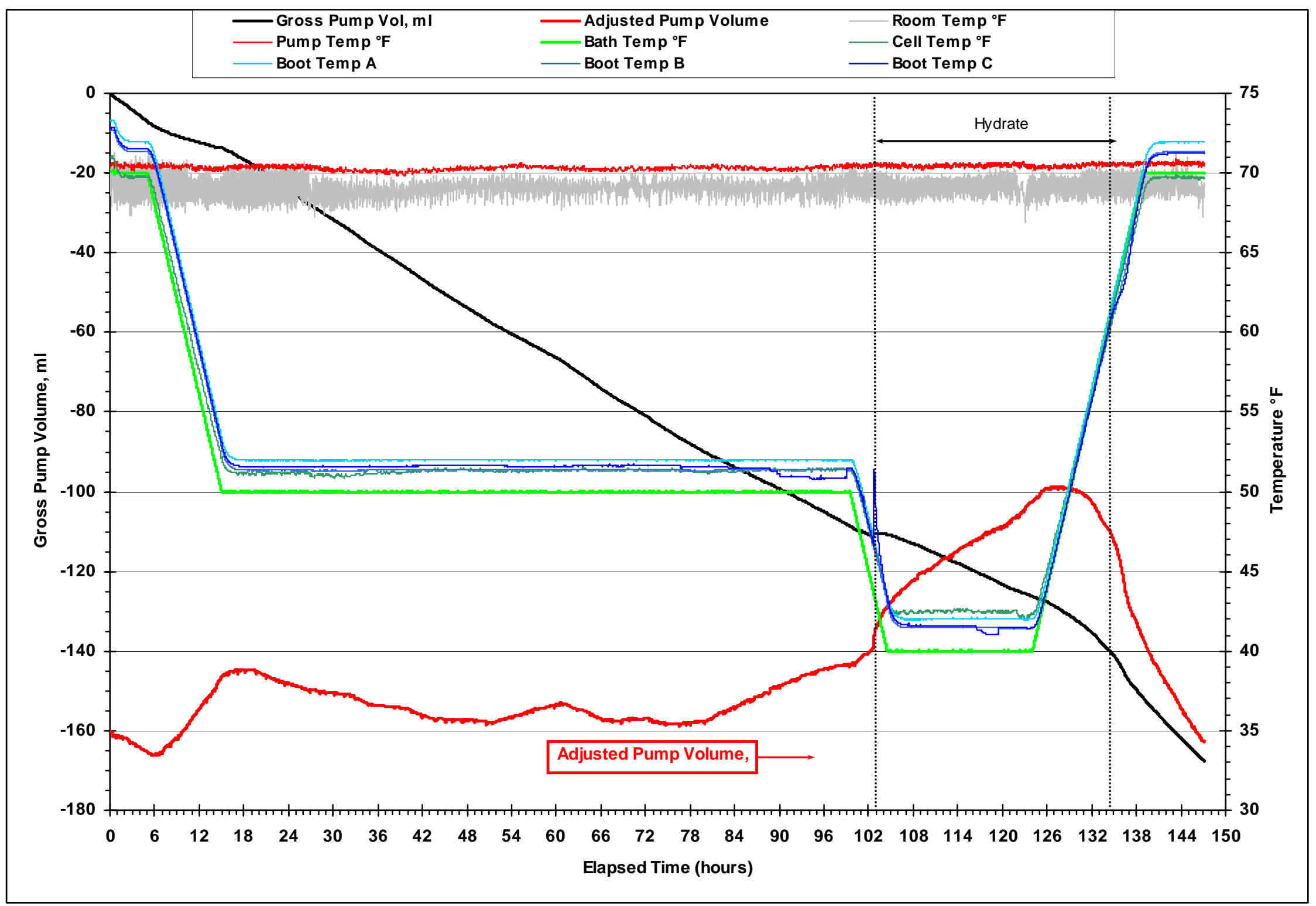


Figure 3.1.11. Test 3: Core Resistance versus Elapsed Time

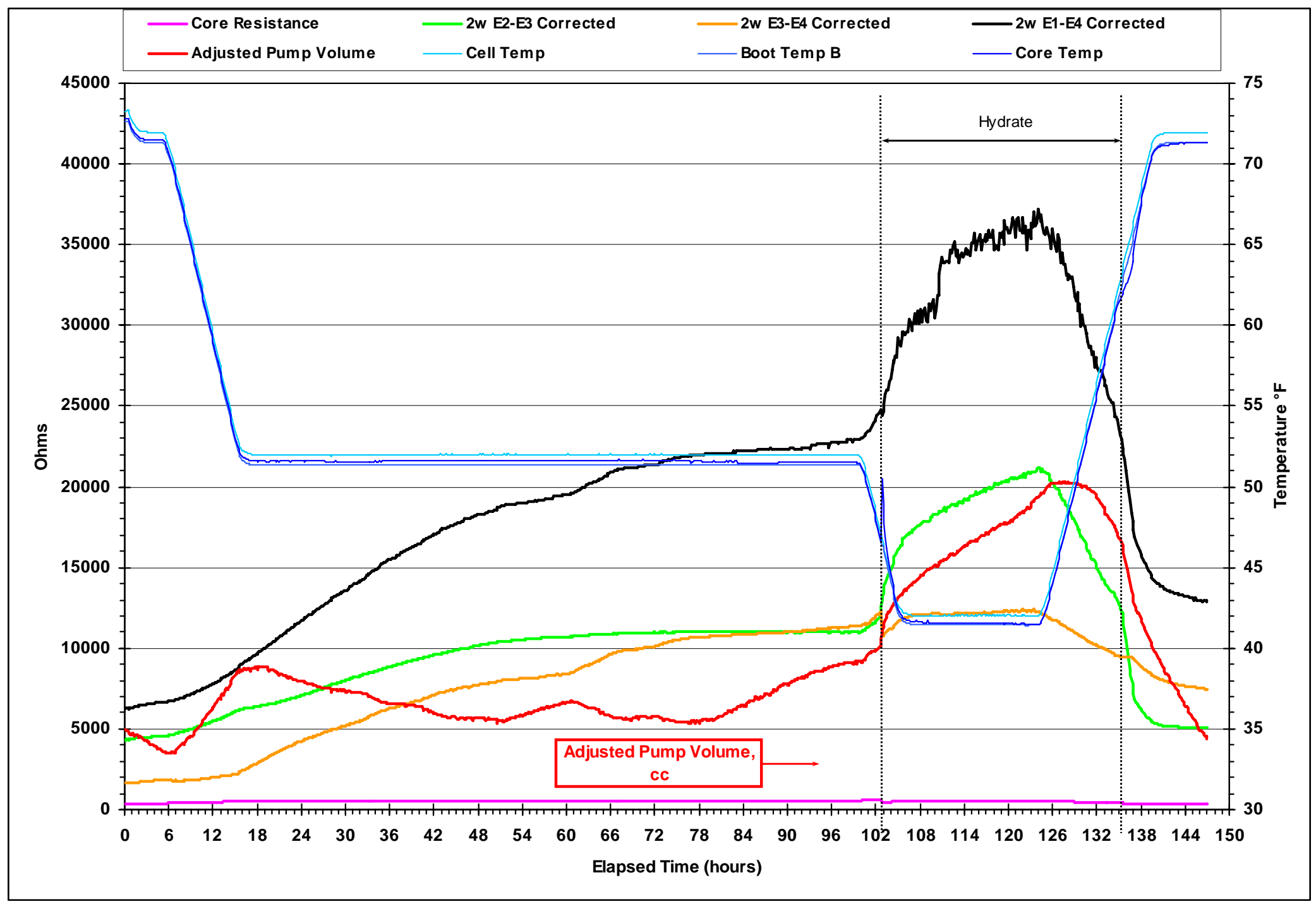


Figure 3.1.12. Test 3: CT Scan Results Before Cooling
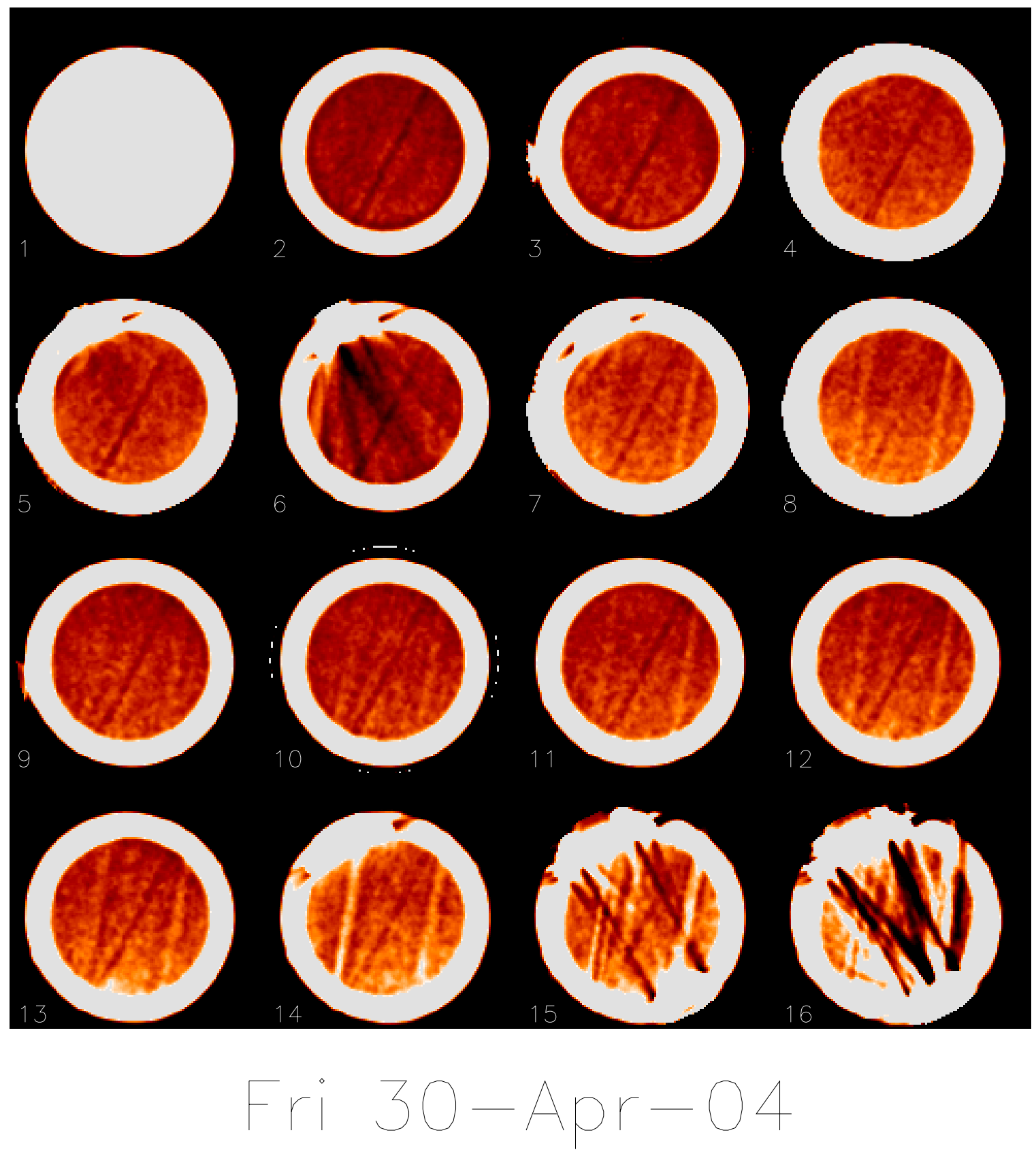

$5 \mathrm{~mm}$ slice spacing

$5 \mathrm{~mm}$ slice thickness 
Figure 3.1.13. Test 3: CT Scan Results After Hydrate Formation

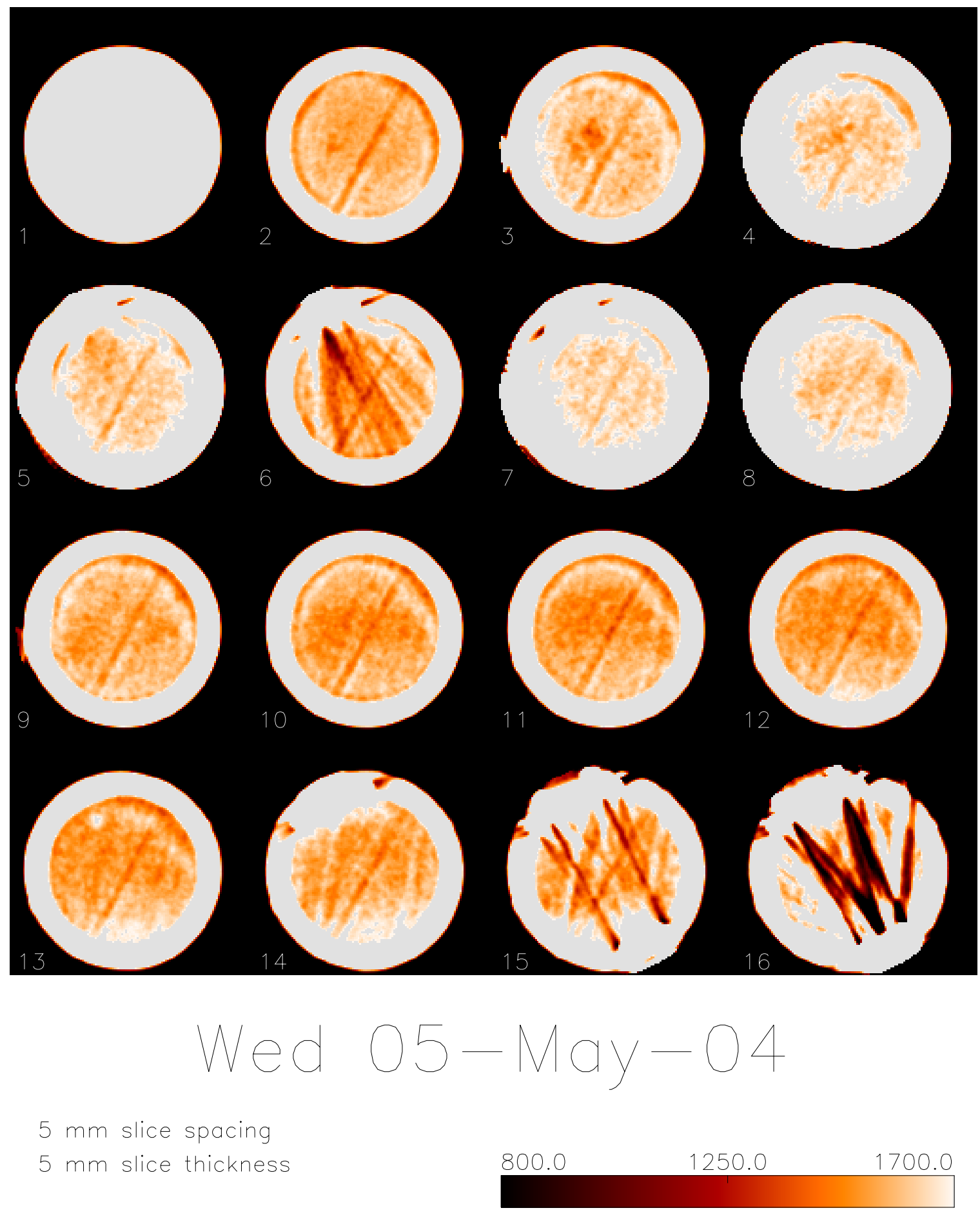


Figure 3.1.14. Test 3: CT Scan Results After Dissociation

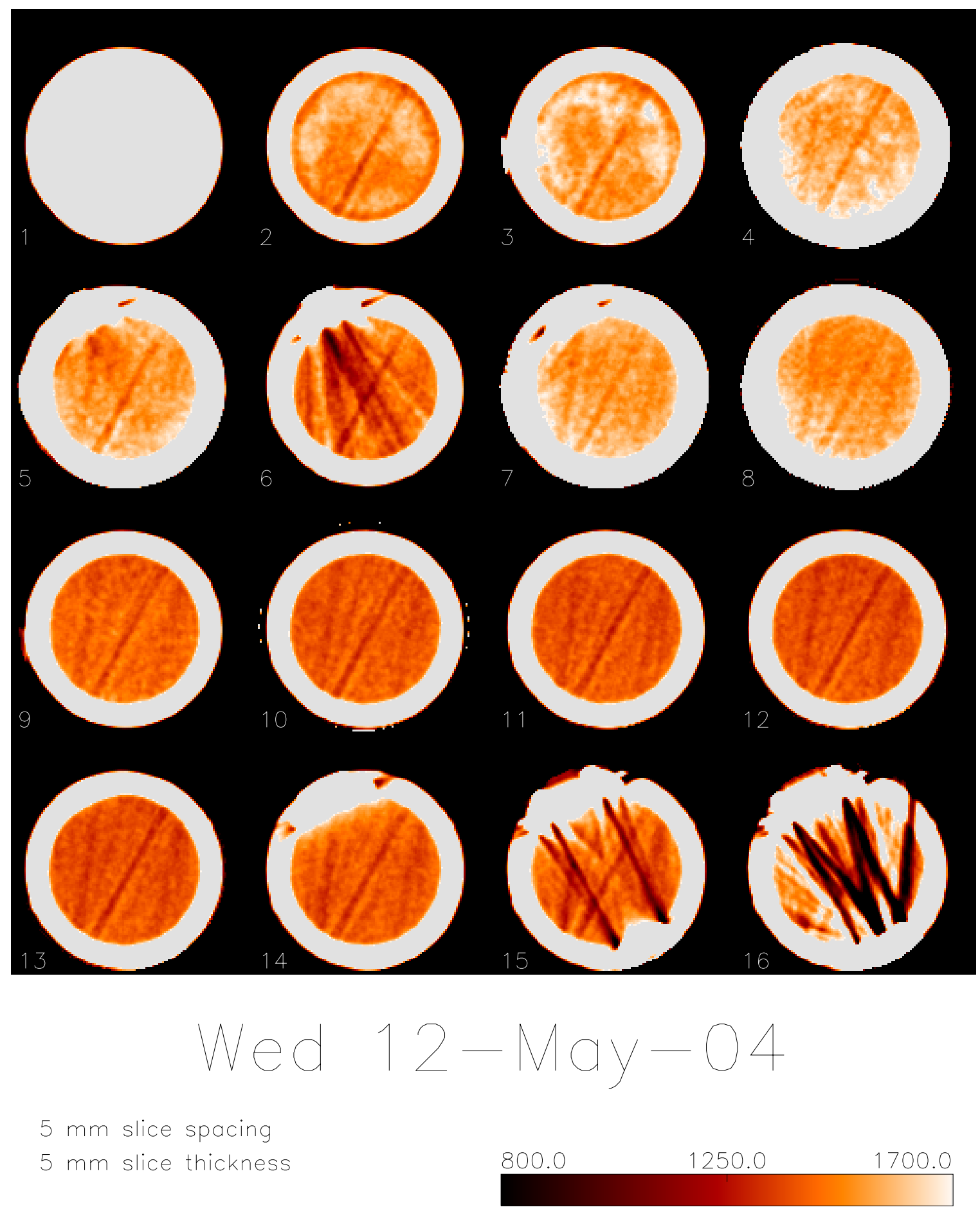




\subsubsection{Hydrate Formation in QF10 Medium Permeability Core (Test 4)}

Core Material:

Permeability (md):

Hydrate Formation Procedure:

Hydrate Dissociation Procedure:
QF10N

29,000

Constant methane $\mathrm{P}$, decreasing $\mathrm{T}$

Constant $P$, increasing $T$

This was first test using a medium porosity core. A clean and dry 9.8 inch $(24.9 \mathrm{~cm})$ long by 1.5 inch diameter piece of QF-10 core material was mounted in the jacket and assembled in the core holder with the acoustical sensors, resistivity electrodes and RTD temperature sensors. A net confining stress (NCS) of 500 psi was applied to the core. The core was saturated with $6 \% \mathrm{Nal}$ brine. The pore volume of the core was $101 \mathrm{cc}$ and the porosity was $35 \%$ of pore volume. CT scans were made of the core in the dry and brine saturated conditions. The core was de-saturated with methane gas to $34 \%$ water saturation, leaving $67 \mathrm{cc}$ of gas in the core. The pore pressure was raised to 2,500 psi with methane while simultaneously raising the overburden pressure to $3,000 \mathrm{psi}$, maintaining a constant 500 psi NCS. Once at pressure, the system was leak tested for 3 days prior to the start of the test. All of these operations were carried out at room temperature.

A constant pressure test to form and then dissociate hydrate in the QF-10 core was begun. The cell was first cooled to $60^{\circ} \mathrm{F}$ at the rate of $2^{\circ} \mathrm{F} / \mathrm{hr}$. and allowed to equilibrate at that temperature for 3 days. The cell was then cooled to $51^{\circ} \mathrm{F}$ at the rate of $2^{\circ} \mathrm{F} / \mathrm{hr}$. and allowed to equilibrate over night. Figure 3.1.15 shows the temperatures, pump volume of gas injected and pressures for the entire test. The cell temperature was again lowered at the rate of $2^{\circ} \mathrm{F} / \mathrm{hr}$.

Hydrate formation began at $45^{\circ} \mathrm{F}$. Like the previous test, the core temperature measured by the RTDs embedded in the jacket showed a sharp increase at the onset of hydrate formation. Though the largest increase in temperature was seen by RTD-C, all three RTDs indicated a substantial rise in temperature at the onset of hydrate formation. The RTD-C is located near the gas supply end of the core. The core temperatures stabilized at $42^{\circ} \mathrm{F}$ about 5 hours after the start of hydrate formation. At the onset of hydrate formation as indicated by the RTDs, the pump began rapidly injecting gas (pump volume increasing) and the pore pressure began declining. The pore pressure continued to decline for 3 hours reaching a low of $2,400 \mathrm{psi}$, indicating the closed end of the core had become partially isolated from the gas supply end. The pore pressure, like the temperature, recovered about 5 hours after the start of hydrate formation. The pump continued to inject gas at a slower rate for 5 days (at about 240 total hours) and then became stable after injecting about $32 \mathrm{cc}$ of gas.

At the onset of hydrate formation, the resistance between the E1 and E2 electrodes (Figure 3.1.16) sharply increased while the E2-E3 resistance began a slower increase. This also suggests that the hydrate formed first near the supply end of the core. The core resistance continued to increase for 2 days after the start of hydrate formation, then declined and became stable. 
After 7 days of hydrate formation, the core pressure, resistances and the pump volume had become stable. Hydrate dissociation was initiated by increasing the core temperature in $5^{\circ} \mathrm{F}$ increments. Unlike hydrate formation, the dissociation occurred at several temperatures over a prolonged period of time. During the first temperature ramp to about $47^{\circ} \mathrm{F}$ the pore pressure remained stable and the pump volume decreased (backed up) about 2cc, indicating gas expansion. This was consistent with the amount of gas expansion expected due to temperature change. The pore pressure showed a 40 psi increase when the core reached about $50^{\circ} \mathrm{F}$ indicating hydrate dissociation. At each subsequent temperature increase the pump backed up significantly more than could be expected from the temperature increase, and then stabilized, indicating some partial dissociation occurred at each temperature change. The two largest volume changes occurred during the $56^{\circ} \mathrm{F}-60^{\circ} \mathrm{F}$ and $60^{\circ} \mathrm{F}-65^{\circ} \mathrm{F}$ temperature ramps indicating most of the dissociation took place at these temperatures. Figure 3.1.15 shows that the core temperatures increased more slowly than the cell temperatures during these ramps, which is consistent with the endothermic nature of hydrate dissociation. There was no significant change after $65^{\circ} \mathrm{F}$ indicating the dissociation was completed.

Acoustic velocity was also collected through the course of the test. As shown in Figure 3.1.17, the velocity of the core increased as the hydrates were formed at around $45^{\circ} \mathrm{F}$; from $3150 \mathrm{~m} / \mathrm{sec}$ to 3650 $\mathrm{m} / \mathrm{sec}$ and equilibrated at $3585 \mathrm{~m} / \mathrm{sec}$. As the temperature of the core was raised, the hydrates started dissociation and the acoustic velocity started to go down. When the core came back to the original condition of $70^{\circ} \mathrm{F}$, the velocity dropped to about $3220 \mathrm{~m} / \mathrm{sec}$. Before hydrate formation and after hydrate dissociation at $70^{\circ} \mathrm{F}$, acoustic velocity increased by $70 \mathrm{~m} / \mathrm{sec}$. The increase in velocity could be attributed either to Nal redistribution in the core or because of increased dissolved gas saturation of brine. It is postulated that the increase in acoustic velocity at hydrate formation onset was due to an increase in fluid/solid density present in pore throats. The increase in brine density was possible either due to increased $\mathrm{Nal}$ concentration in the brine during hydrate formation or due to formation of hydrate in the pore throats.

The core was CT scanned at various times during the course of the test. Figure 3.1.18 shows the average CT numbers of each slice along the length of the core during hydrate formation. The positions of the resistivity electrodes are also shown. The average CT numbers in the closed end of the core systematically increased with time indicating an increase in Nal concentration. This suggests that hydrate formed near the supply end of the core and pushed Nal towards the closed end, which is consistent with the temperature and resistance data. During dissociation the redistribution of Nal was reversed.

A CT scan of the core after hydrate formation is shown in Figure 3.1.19. Slices 5 and 22 are in the vicinity of the E1 and E2 electrodes, respectively. These effects were adjusted in later tests. 
Figure 3.1.15. Test 4: Hydrate Formation at Constant Methane Pressure

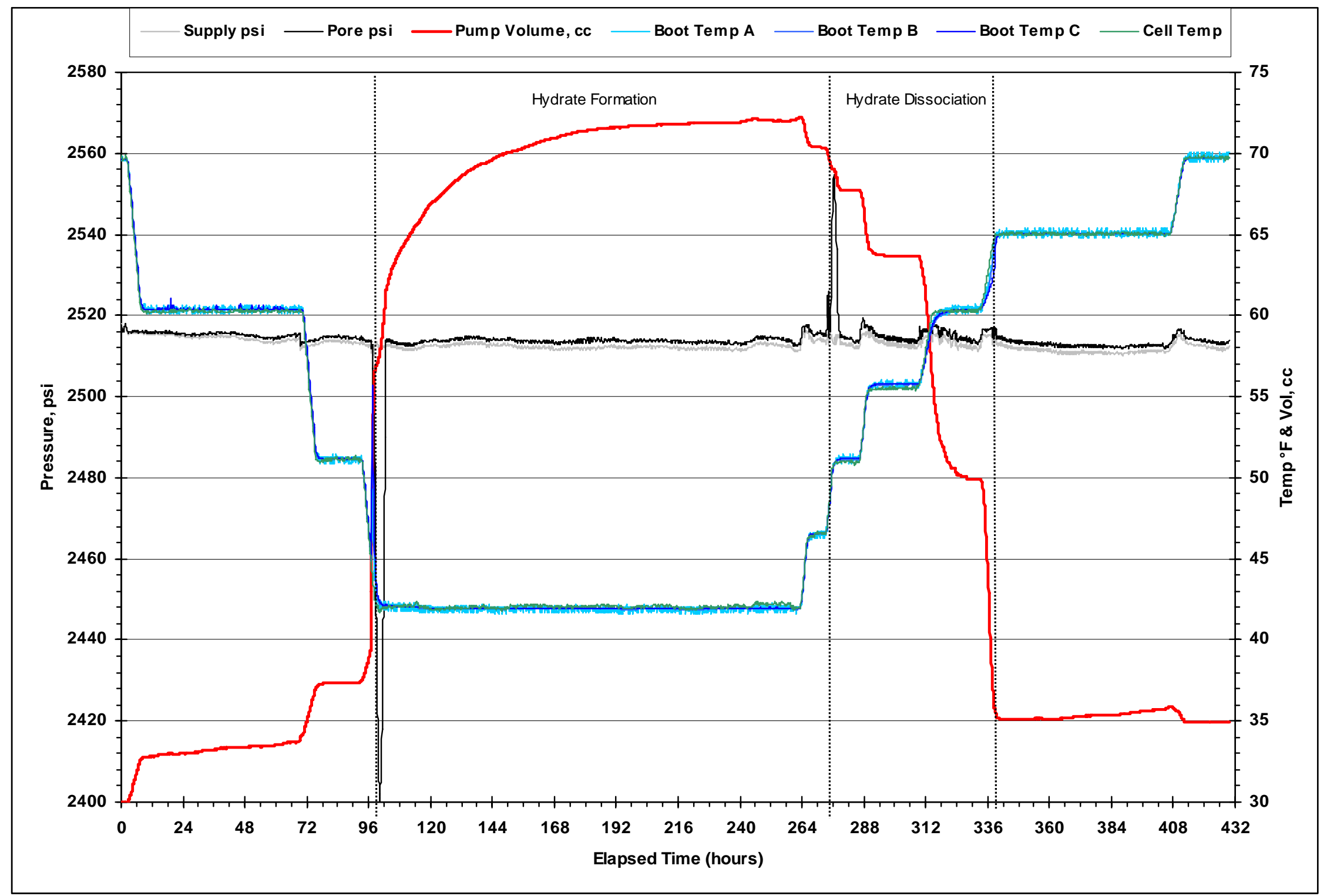


Figure 3.1.16. Test 4: Core Resistance versus Elapsed Time

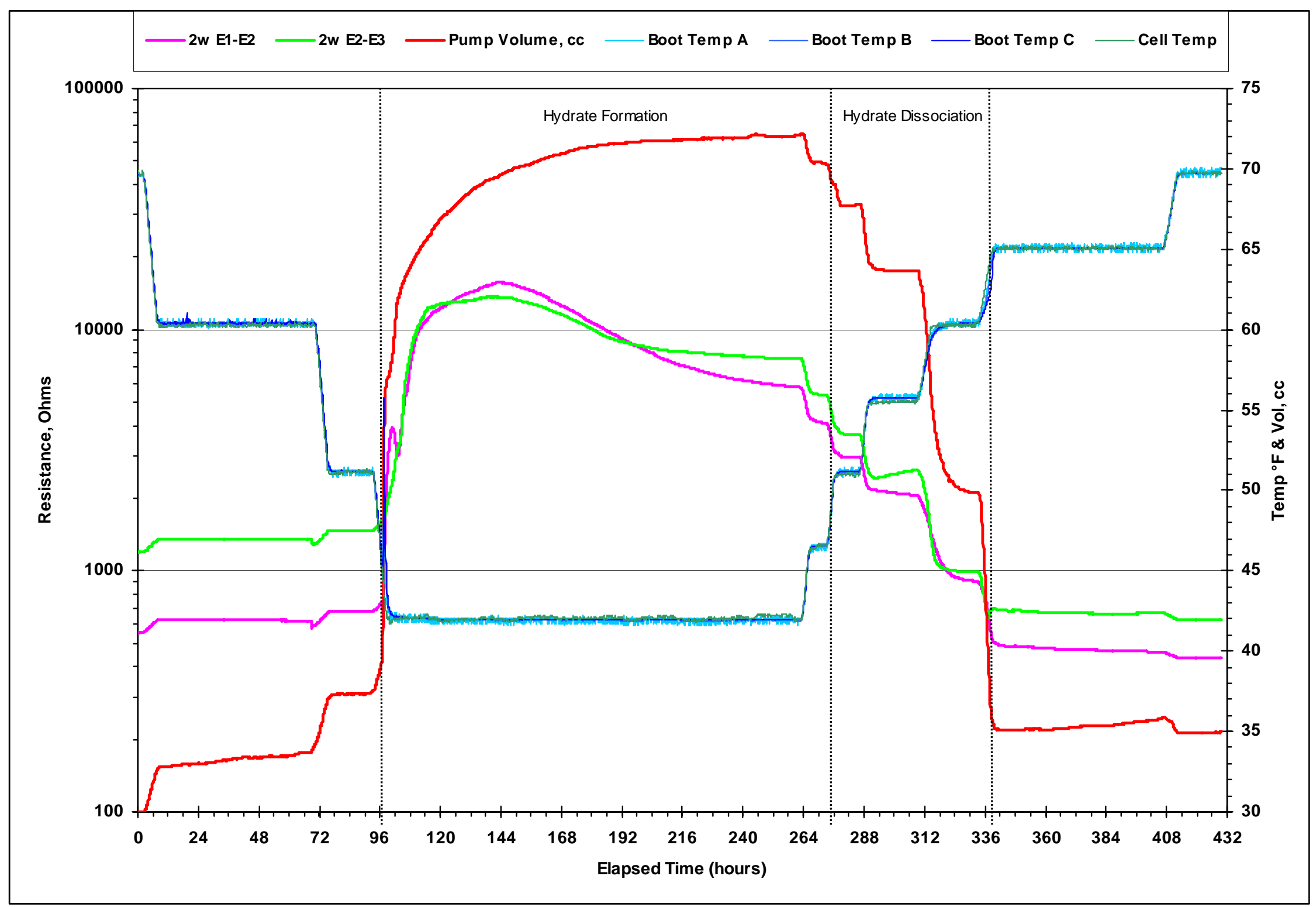


Figure 3.1.17. Test 4: Acoustic Velocity versus Elapsed Time

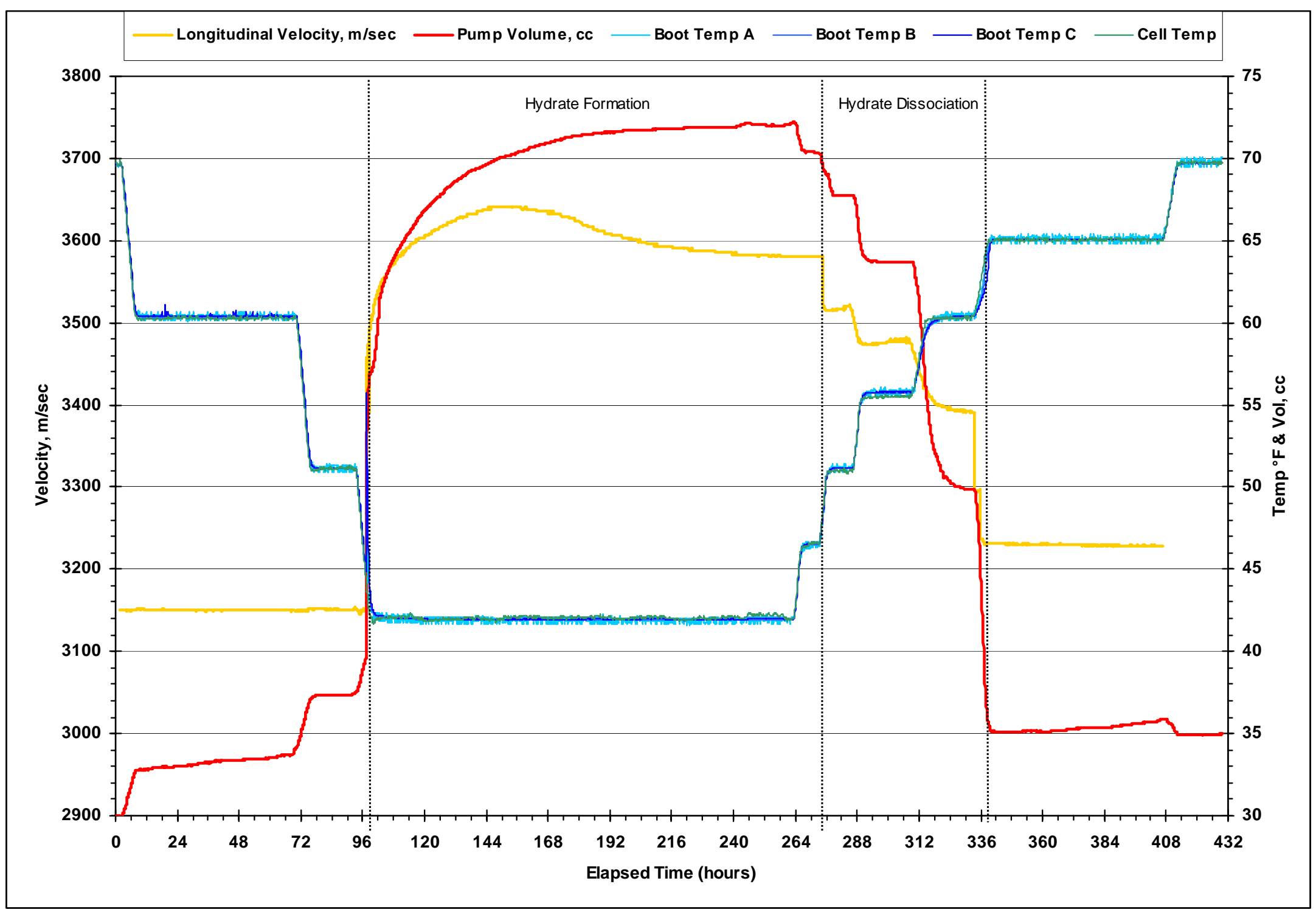


Figure 3.1.18. Test 4: CT Scan Results During Hydrate Formation and Dissociation

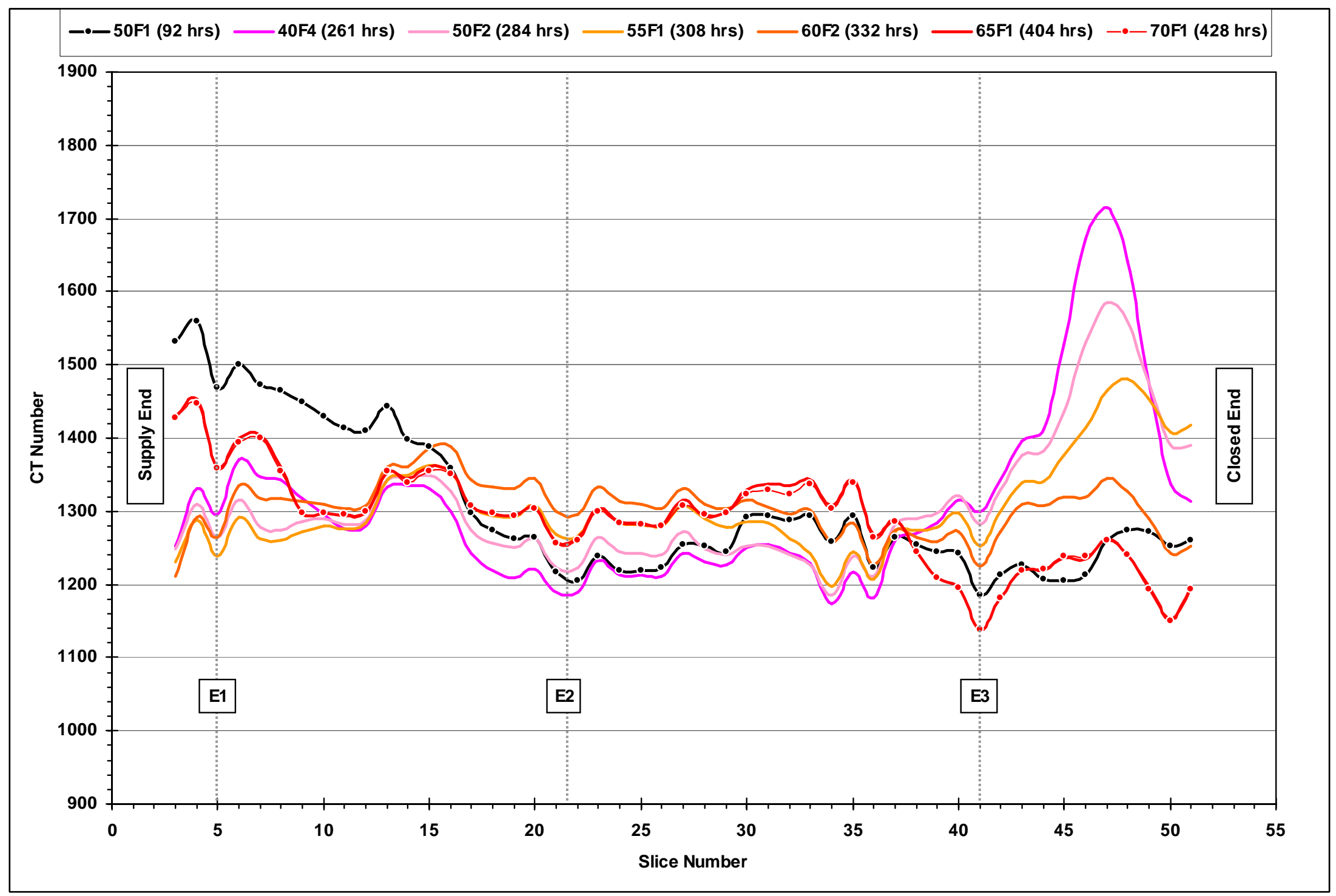


Figure 3.1.19. Test 4: CT Scan Results After Hydrate Formation
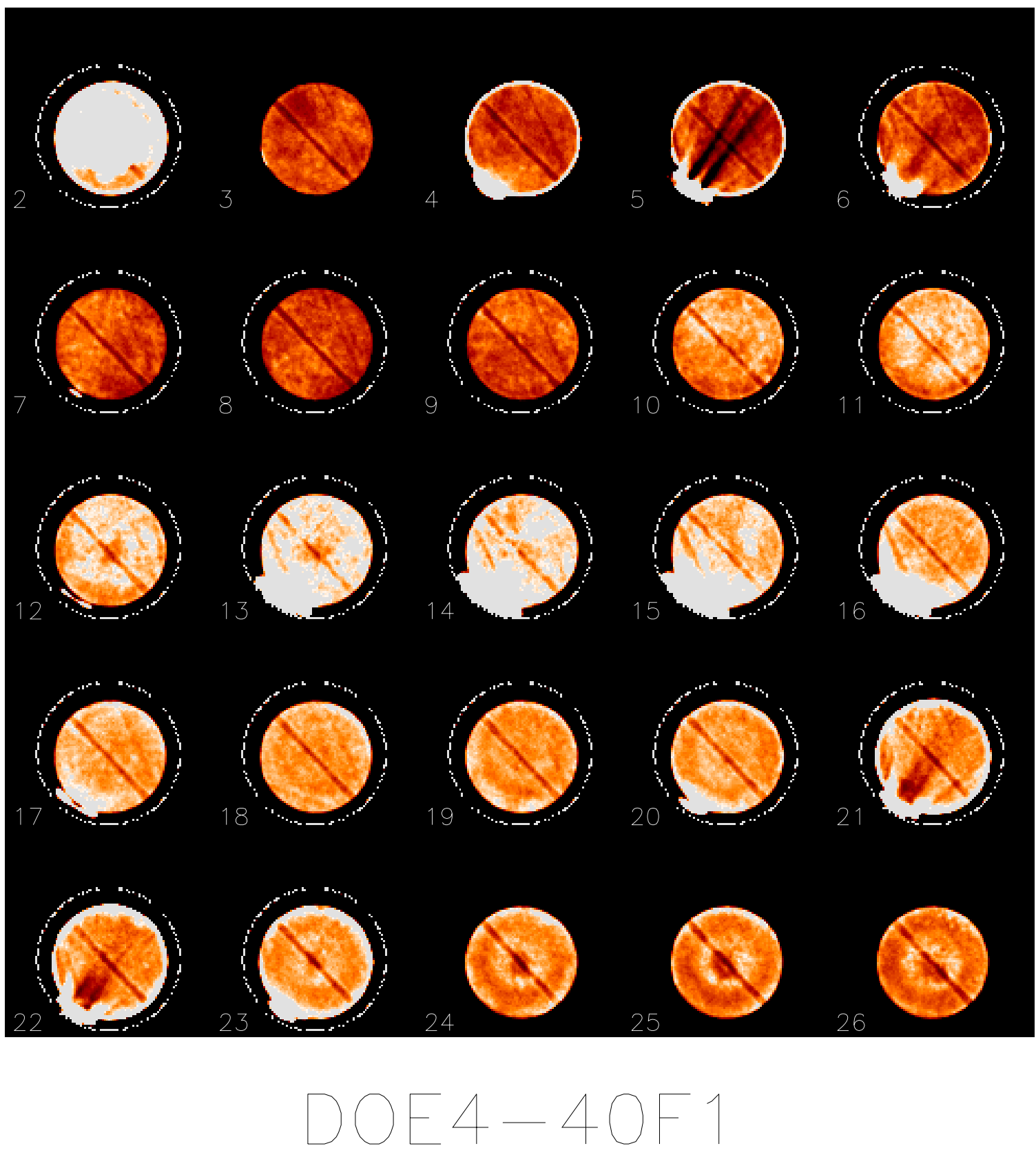

$5 \mathrm{~mm}$ slice spacing

$5 \mathrm{~mm}$ slice thickness 


\subsubsection{Hydrate Formation in QF10 Medium Permeability Core (Test 5)}

Core Material:

Permeability (md):

Hydrate Formation Procedure:

Hydrate Dissociation Procedure:
QF10B

29,000

Constant methane $\mathrm{P}$, decreasing $\mathrm{T}$

Pressure depletion

In previous experiments, dissociation was accomplished by temperature increase. The purpose of this test was to perform hydrate dissociation by decreasing pressure.

A clean and dry 11.96 inch $(30.38 \mathrm{~cm})$ long by 1.5 inch diameter piece of QF-10 core material was mounted in the jacket and assembled in the core holder with the acoustical sensors, resistivity electrodes and RTD temperature sensors. A net confining stress (NCS) of 500 psi was applied to the core. The core was saturated with $6 \% \mathrm{Nal}$ brine. The pore volume of the core was $133 \mathrm{cc}$ and the porosity was $38 \%$ of pore volume. CT scans were made of the core in the dry and brine saturated conditions. The core was de-saturated with methane gas to $34 \%$ water saturation, leaving $67 \mathrm{cc}$ of gas in the core. The pore pressure was raised to 2,500 psi with methane while simultaneously raising the overburden pressure to $3,000 \mathrm{psi}$, maintaining a constant 500 psi NCS. Once at pressure, the system was leak tested for 3 days prior to the start of the test. All of these operations were carried out at room temperature.

A constant pressure test to form and then dissociate hydrate in the QF-10 core was begun. The cell was first cooled to $60^{\circ} \mathrm{F}$ at the rate of $2^{\circ} \mathrm{F} / \mathrm{hr}$. and allowed to equilibrate at that temperature for 3 days. The cell was then cooled to $51^{\circ} \mathrm{F}$ at the rate of $2^{\circ} \mathrm{F} / \mathrm{hr}$. and allowed to equilibrate over night. Figure 3.1.20 shows the pressures and temperatures during the test. The cell temperature was again lowered at the rate of $2^{\circ} \mathrm{F} / \mathrm{hr}$.

Hydrate formation began at $45^{\circ} \mathrm{F}$. Like the previous test, the core temperature measured by the RTDs embedded in the jacket showed a sharp increase at the onset of hydrate formation. The core temperatures stabilized at $42^{\circ} \mathrm{F}$ about 5 hours after the start of hydrate formation. At the onset of hydrate formation as indicated by the RTDs, the pump began rapidly injecting gas (pump volume increasing) and the pore pressure began declining.

At the onset of hydrate formation, the resistance between the E1 and E2 electrodes sharply increased while the E2-E3 resistance began a slower increase as shown in Figure 3.1.21. This also suggested that the hydrate formed first near the supply end of the core. The core resistance continued to increase for 2 days after the start of hydrate formation, then declined and became stable. 
After 3 days of hydrate formation, the core pressure, resistances and the pump volume had become stable. Sodium Chloride brine was injected into the core to determine relative permeability to brine. High injection pressures were seen so brine injection was stopped.

Acoustics data throughout the test are shown in Figure 3.1.22. Acoustic velocity increased (i.e., delay time decreased) during hydrate formation.

A CT scan of the core after hydrate formation is shown in Figure 3.1.23. The red color indicates areas of gas saturation, and the white color indicates areas of brine saturation. Dissociation from the core was completed by depressurization. 
Figure 3.1.20. Test 5: Hydrate Formation at Constant Methane Pressure

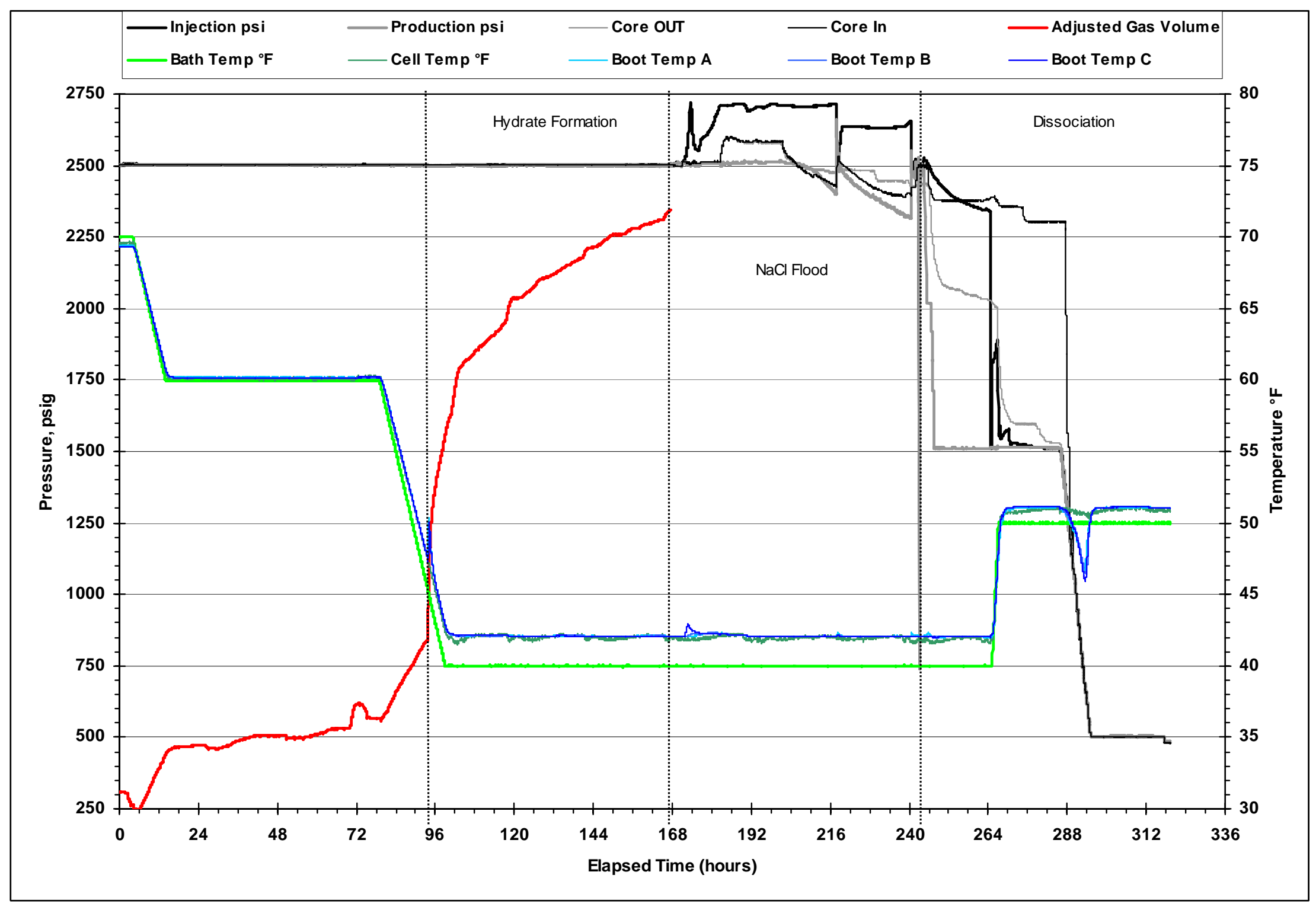


Figure 3.1.21. Test 5: Core Resistance versus Elapsed Time

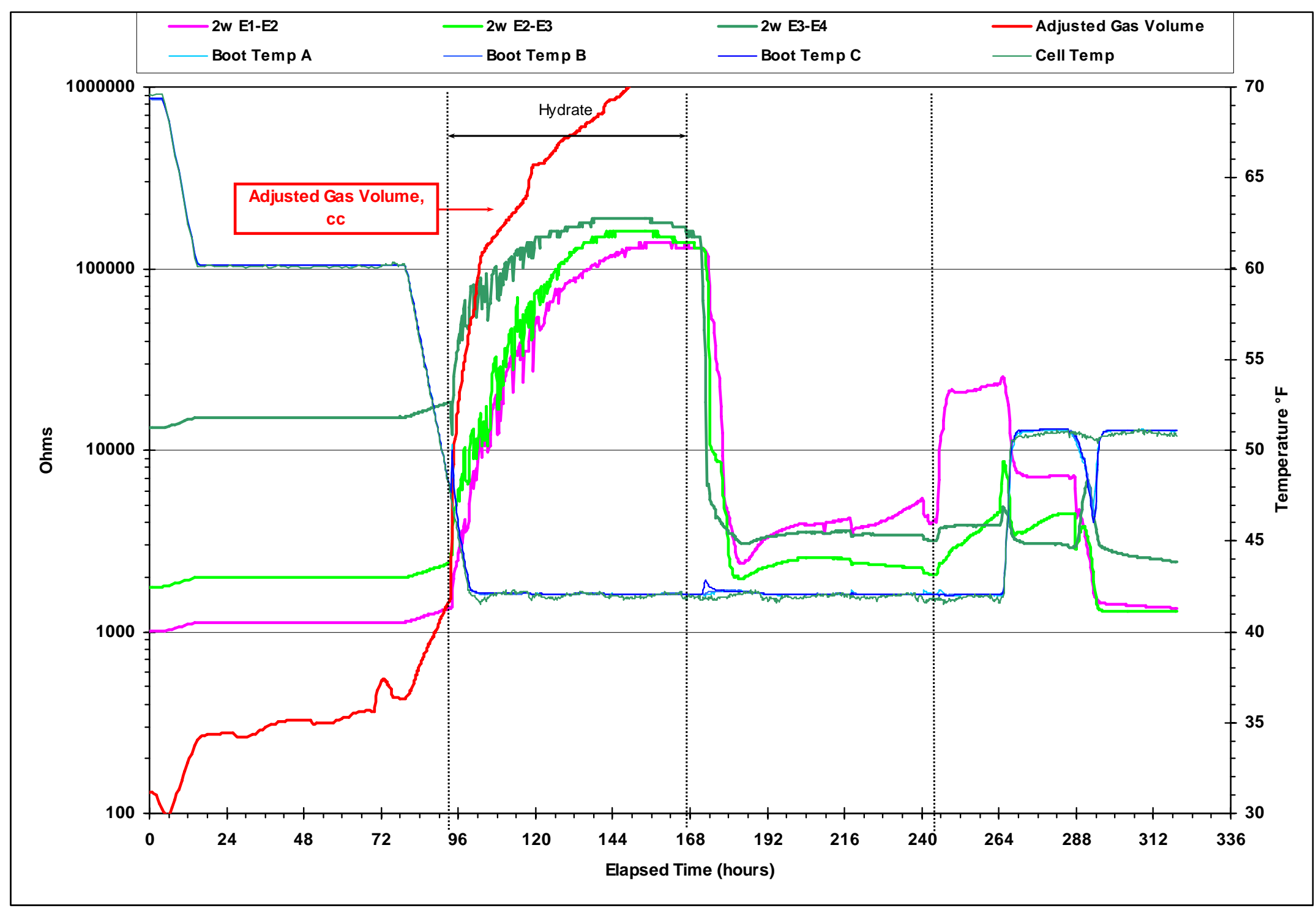


Figure 3.1.22. Test 5: Acoustic Delay Time versus Elapsed Time

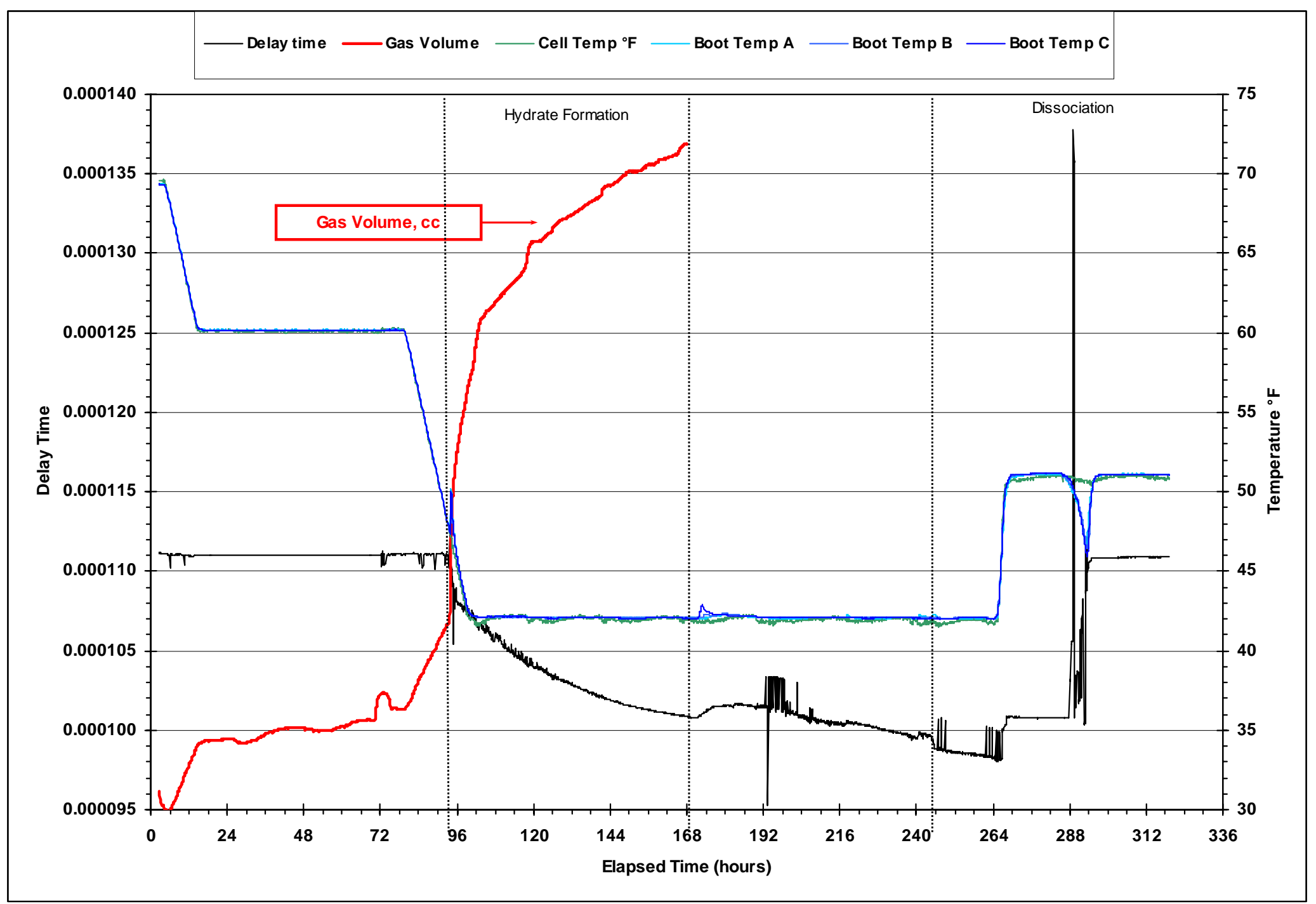


Figure 3.1.23. Test 5: CT Scan Results After Hydrate Formation

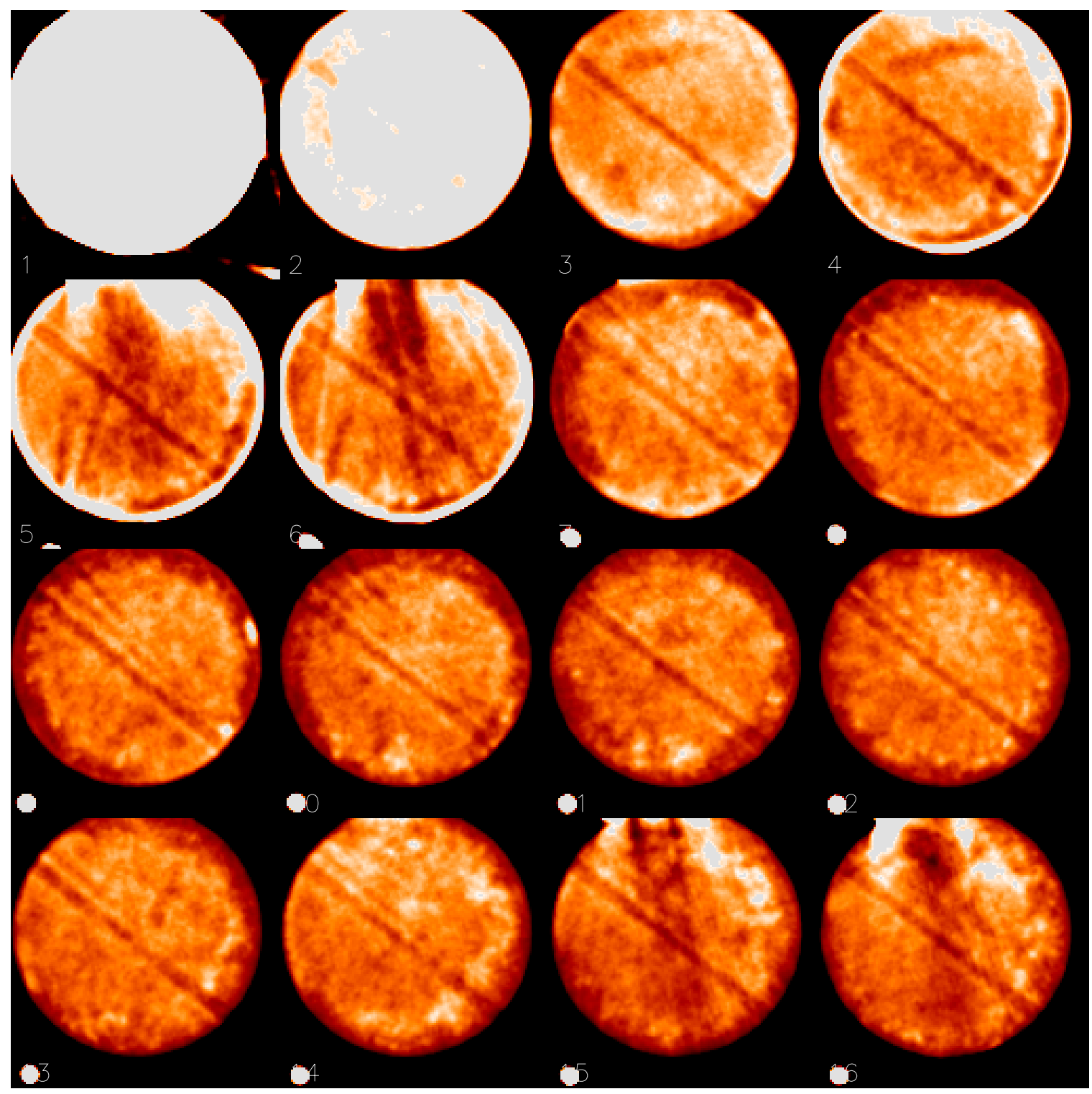

\section{DOE5-4OFG}

$5 \mathrm{~mm}$ slice thickness 


\subsubsection{Hydrate Formation in QF10 Medium Permeability Core (Test 6)}

Core Material:

Permeability (md):

Hydrate Formation Procedure:

Hydrate Dissociation Procedure:
QF10B

29,000

Constant methane $\mathrm{P}$, decreasing $\mathrm{T}$

Constant $P$, increasing $T$

Test 6 was a confirmation of Test 4. A clean and dry 11.96 inch $(30.38 \mathrm{~cm})$ long by 1.5 inch diameter piece of QF10 core material was mounted in the jacket and assembled in the core holder with the acoustical sensors, resistivity electrodes and RTD temperature sensors. A net confining stress (NCS) of 500 psi was applied to the core. The core was saturated with $6 \% \mathrm{Nal}$ brine. The pore volume of the core was $133 \mathrm{cc}$ and the porosity was $38 \%$ of pore volume. CT scans were made of the core in the dry and brine saturated conditions. The core was de-saturated with methane gas to $34 \%$ water saturation, leaving $67 \mathrm{cc}$ of gas in the core. The pore pressure was raised to 2,500 psi with methane while simultaneously raising the overburden pressure to $3,000 \mathrm{psi}$, maintaining a constant $500 \mathrm{psi}$ NCS. Once at pressure, the system was leak tested for 3 days prior to the start of the test. All of these operations were carried out at room temperature.

A constant pressure test to form and then dissociate hydrate in the QF-10 core was begun. The cell was first cooled to $60^{\circ} \mathrm{F}$ at the rate of $2^{\circ} \mathrm{F} / \mathrm{hr}$. and allowed to equilibrate at that temperature for 3 days. The cell was then cooled to $51^{\circ} \mathrm{F}$ at the rate of $2^{\circ} \mathrm{F} / \mathrm{hr}$. and allowed to equilibrate over night. Figure 3.1.24 shows the temperatures, pump volume of gas injected and pressures for the entire test. The cell temperature was again lowered at the rate of $2^{\circ} \mathrm{F} / \mathrm{hr}$.

Hydrate formation began at $45^{\circ} \mathrm{F}$. Like the previous test, the core temperature measured by the RTDs embedded in the jacket showed a sharp increase at the onset of hydrate formation. The core temperatures stabilized at $42^{\circ} \mathrm{F}$ about 5 hours after the start of hydrate formation. At the onset of hydrate formation, as indicated by the RTDs, the pump began rapidly injecting gas (pump volume increasing) and the pore pressure began declining.

At the onset of hydrate formation, the resistance between the E1 and E2 electrodes sharply increased while the E2-E3 resistance began a slower increase (Figure 3.1.25). This also suggests that the hydrate formed first near the supply end of the core. The core resistance continued to increase for 2 days after the start of hydrate formation, then declined and became stable.

Acoustic velocity was also collected through the course of the test. Similar to Test 4 , acoustic velocity increased (delay time decreased) as shown in Figure 3.1.26. A CT scan of the core after hydrate formation is shown in Figure 3.1.27. The red color indicates areas of gas saturation, and the white color indicates areas of brine saturation. 
After 2 days of hydrate formation, the core pressure, resistances and the pump volume had become stable. Sodium Chloride brine was injected into the core to determine relative permeability to brine. High injection pressures were seen so brine injection was stopped. Dissociation from the core was completed by increasing the temperature. 
Figure 3.1.24. Test 6: Hydrate Formation at Constant Methane Pressure

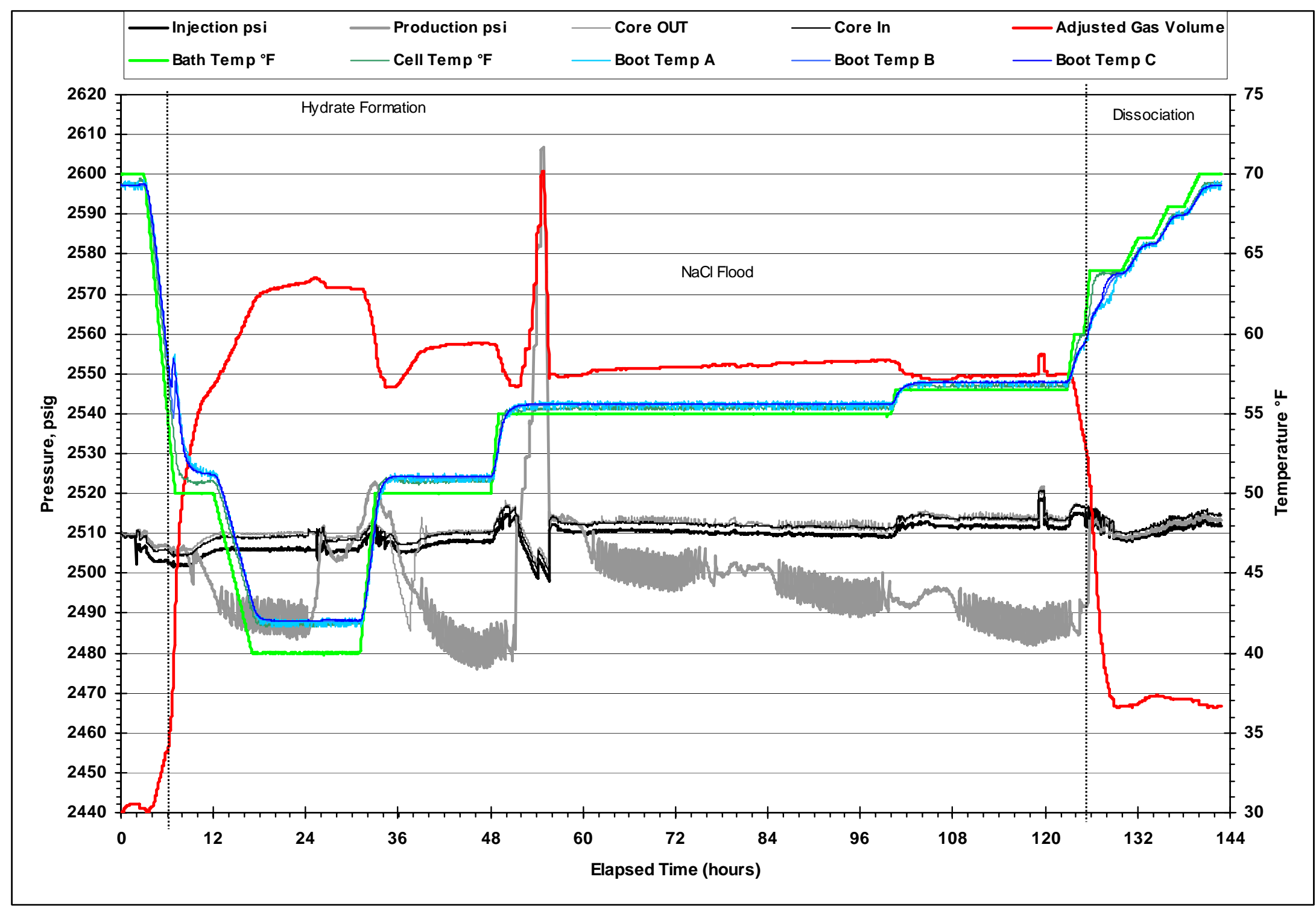


Figure 3.1.25. Test 6: Core Resistance versus Elapsed Time

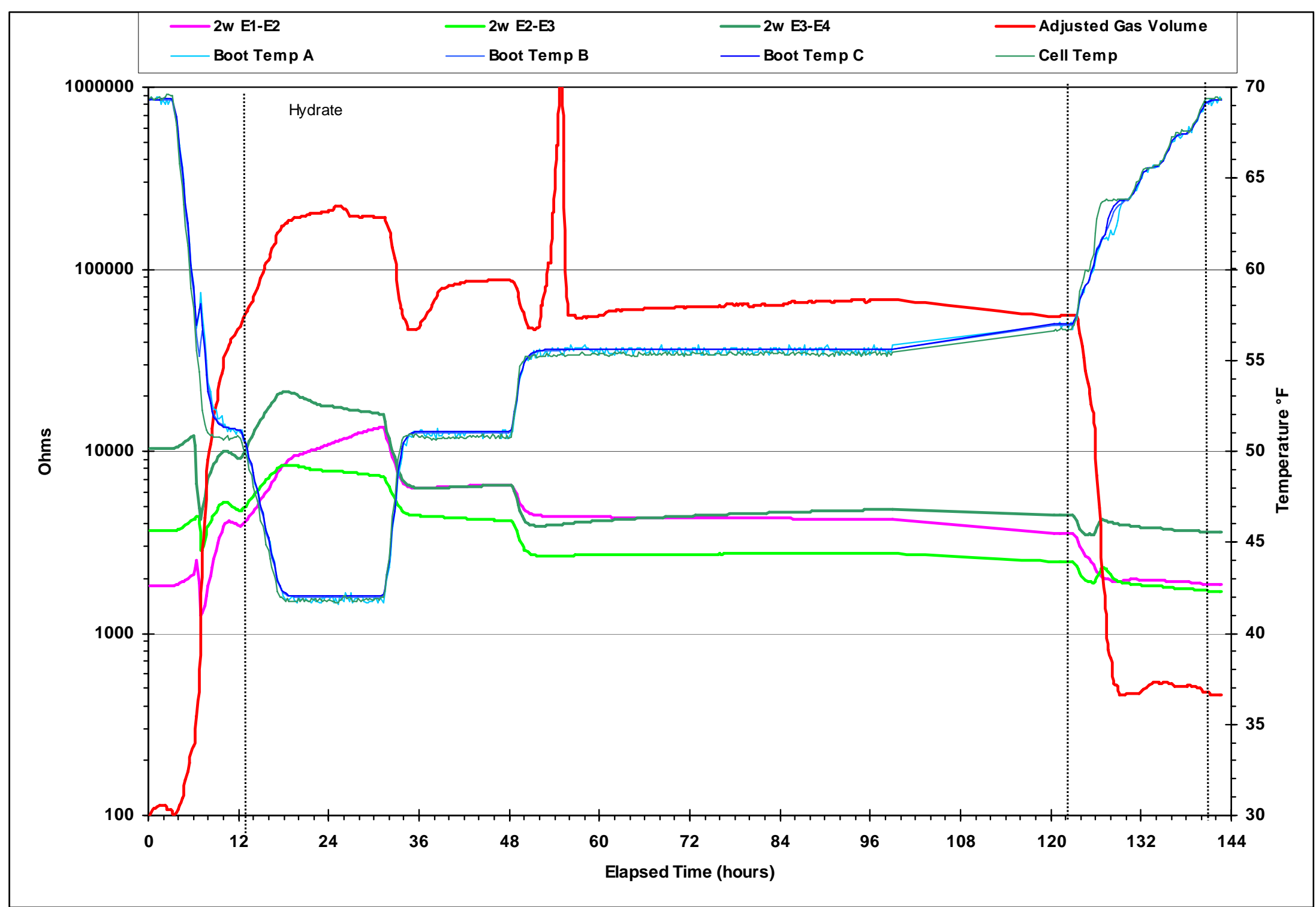


Figure 3.1.26. Test 6: Acoustic Delay Time versus Elapsed Time

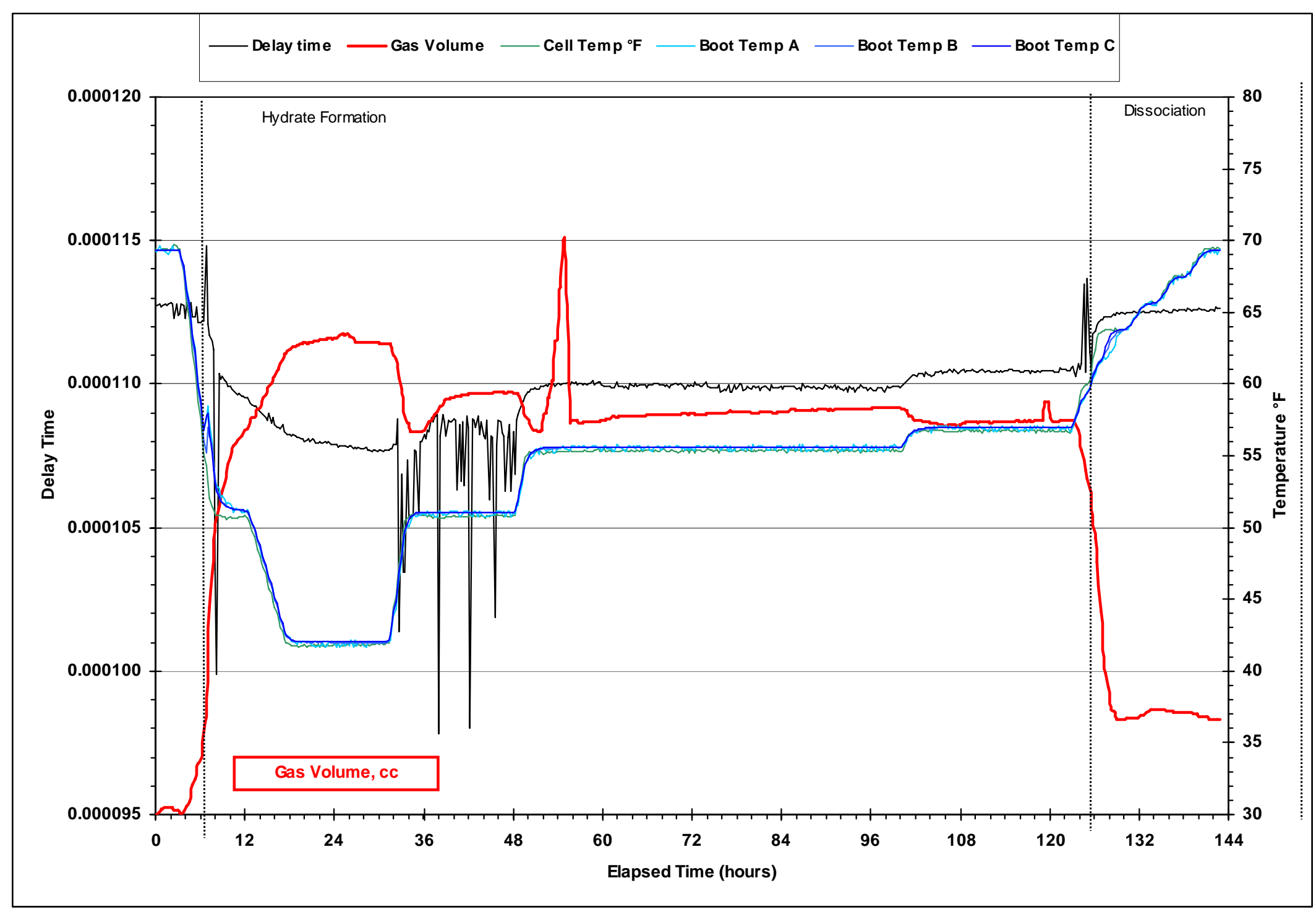


Figure 3.1.27. Test 6: CT Scan Results after Hydrate Formation
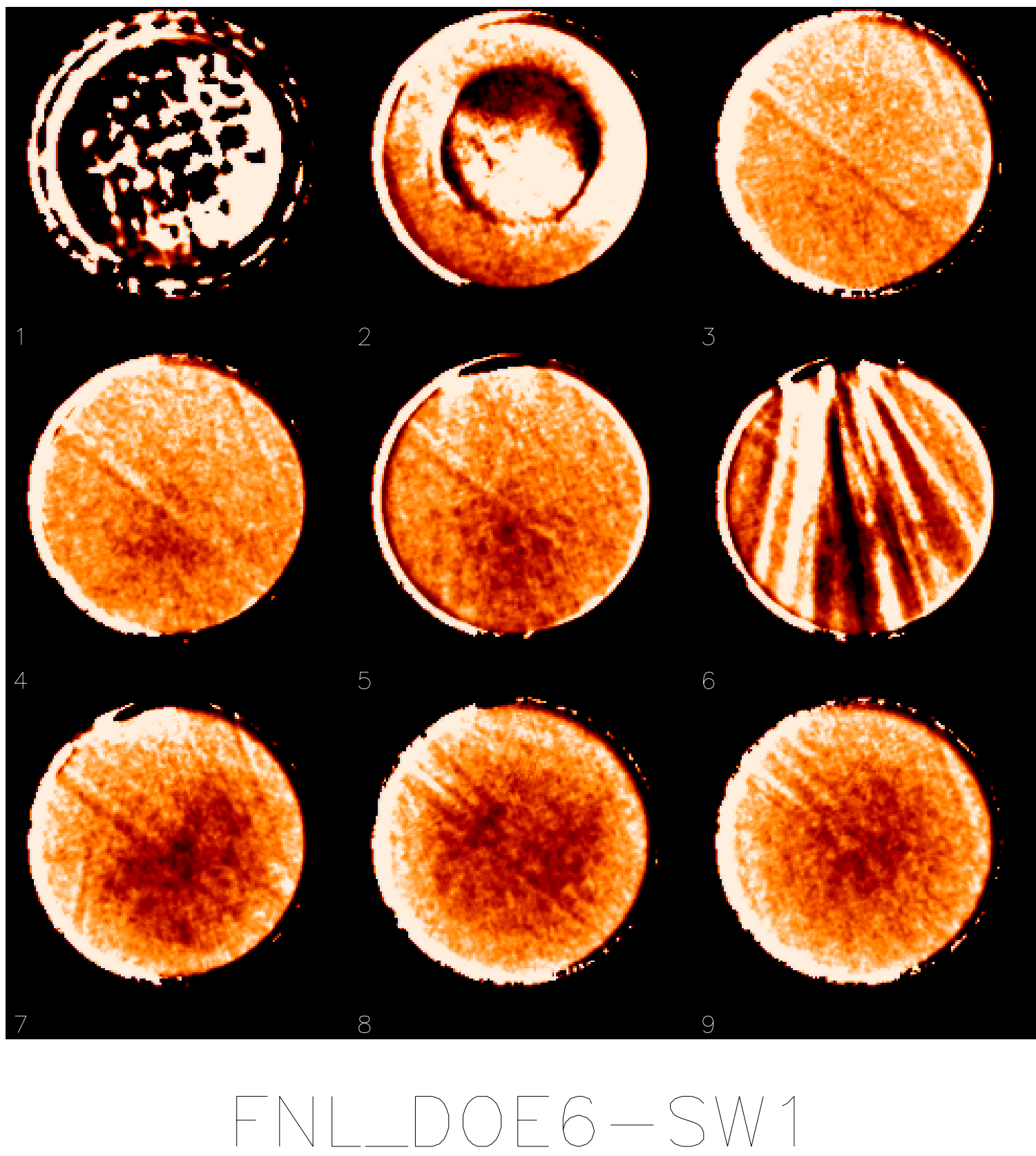

$6 \mathrm{~mm}$ slice spacing

$5 \mathrm{~mm}$ slice thickness 


\subsubsection{Hydrate Formation in QF10 Medium Permeability Core (Test 7)}

Core Material:

Permeability (md):

Hydrate Formation Procedure:

Hydrate Dissociation Procedure:
QF10B

29,000

Constant methane $\mathrm{P}$, decreasing $\mathrm{T}$

Pressure depletion

Test 7 was the first repetition of Test 5 . A clean and dry 11.96 inch $(30.38 \mathrm{~cm})$ long by 1.5 inch diameter piece of QF-10 core material was mounted in the jacket and assembled in the core holder with the acoustical sensors, resistivity electrodes and RTD temperature sensors. A net confining stress (NCS) of 500 psi was applied to the core. The core was saturated with $6 \% \mathrm{Nal}$ brine. The pore volume of the core was $133 \mathrm{cc}$ and the porosity was 38\% of pore volume. CT scans were made of the core in the dry and brine saturated conditions. The core was de-saturated with methane gas to $34 \%$ water saturation, leaving $67 \mathrm{cc}$ of gas in the core. The pore pressure was raised to 2,500 psi with methane while simultaneously raising the overburden pressure to $3,000 \mathrm{psi}$, maintaining a constant $500 \mathrm{psi}$ NCS. Once at pressure, the system was leak tested for 3 days prior to the start of the test. All of these operations were carried out at room temperature.

A constant pressure test to form and then dissociate hydrate in the QF-10 core was begun. The cell was first cooled to $60^{\circ} \mathrm{F}$ at the rate of $2^{\circ} \mathrm{F} / \mathrm{hr}$. and allowed to equilibrate at that temperature for 3 days. The cell was then cooled to $51^{\circ} \mathrm{F}$ at the rate of $2^{\circ} \mathrm{F} / \mathrm{hr}$. and allowed to equilibrate over night. Figure 3.1.28 shows the temperatures, pump volume of gas injected and pressures for the entire test. The cell temperature was again lowered at the rate of $2^{\circ} \mathrm{F} / \mathrm{hr}$.

Hydrate formation began at $45^{\circ} \mathrm{F}$. Like the Test 5 , the core temperature measured by RTDs embedded in the jacket showed a sharp increase at the onset of hydrate formation. The core temperatures stabilized at $42^{\circ} \mathrm{F}$ about 5 hours after the start of hydrate formation. At the onset of hydrate formation as indicated by the RTDs, the pump began rapidly injecting gas and the pore pressure began declining.

At the onset of hydrate formation, the resistance between the E1 and E2 electrodes sharply increased while the E2-E3 resistance began a slower increase (Figure 3.1.29). This also suggests that the hydrate formed first near the supply end of the core. The core resistance continued to increase for 2 days after the start of hydrate formation then declined and became stable. After 2 days of hydrate formation, the core pressure, resistances and the pump volume had become stable. Sodium Chloride brine was injected into the core to determine relative permeability to brine. High injection pressures were seen so brine injection was stopped.

Similar to Test 5, acoustic data shown in Figure 3.1.30 indicated that acoustic velocity increased (i.e., delay time decreased) during hydrate formation. A CT scan of the core after hydrate formation is shown in Figure 3.1.31. The red color indicates areas of gas saturation, and the white color indicates areas of brine saturation. Dissociation from the core was completed by pressure depletion. 
Figure 3.1.28. Test 7: Hydrate Formation at Constant Methane Pressure

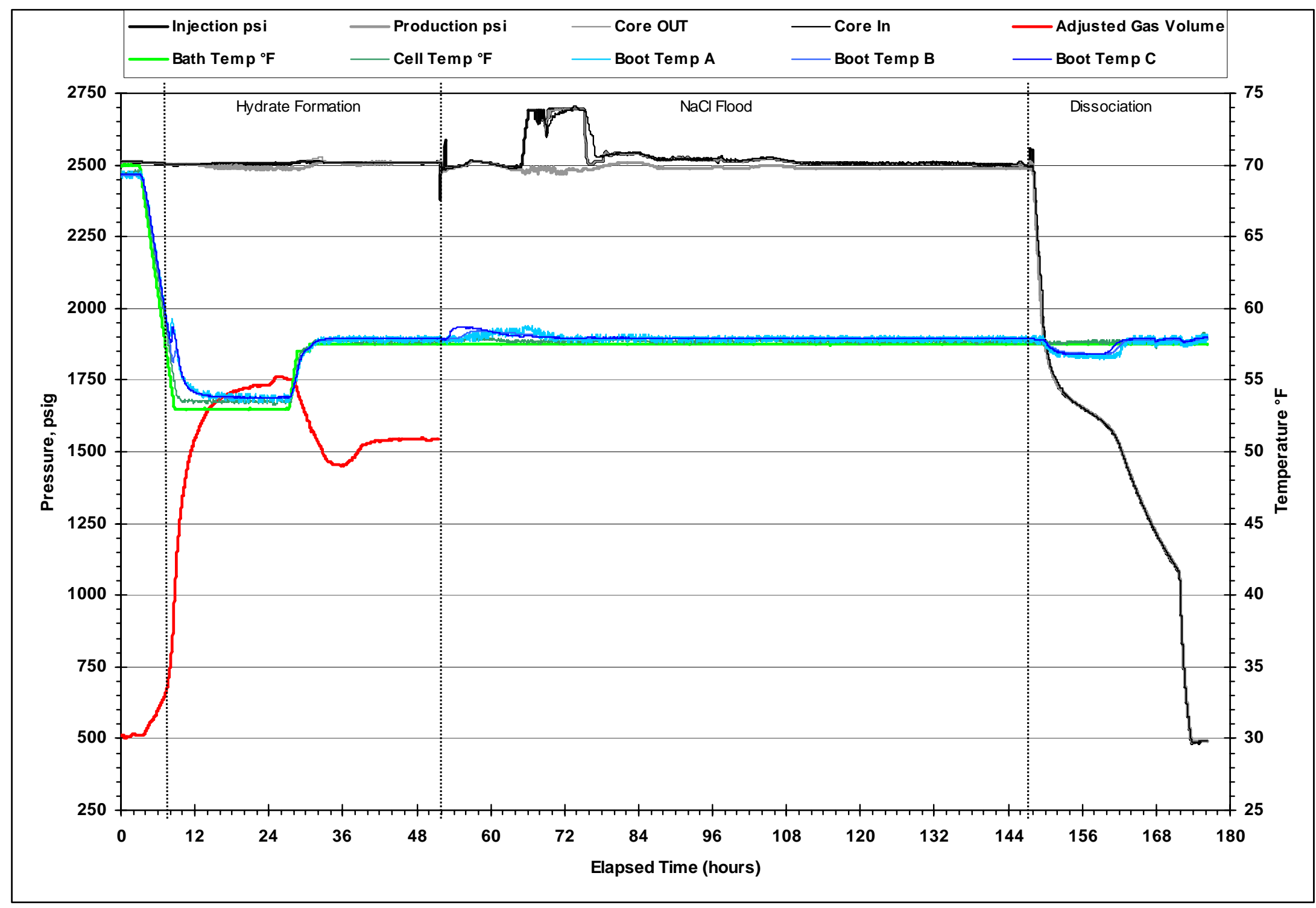


Figure 3.1.29. Test 7: Core Resistance versus Elapsed Time

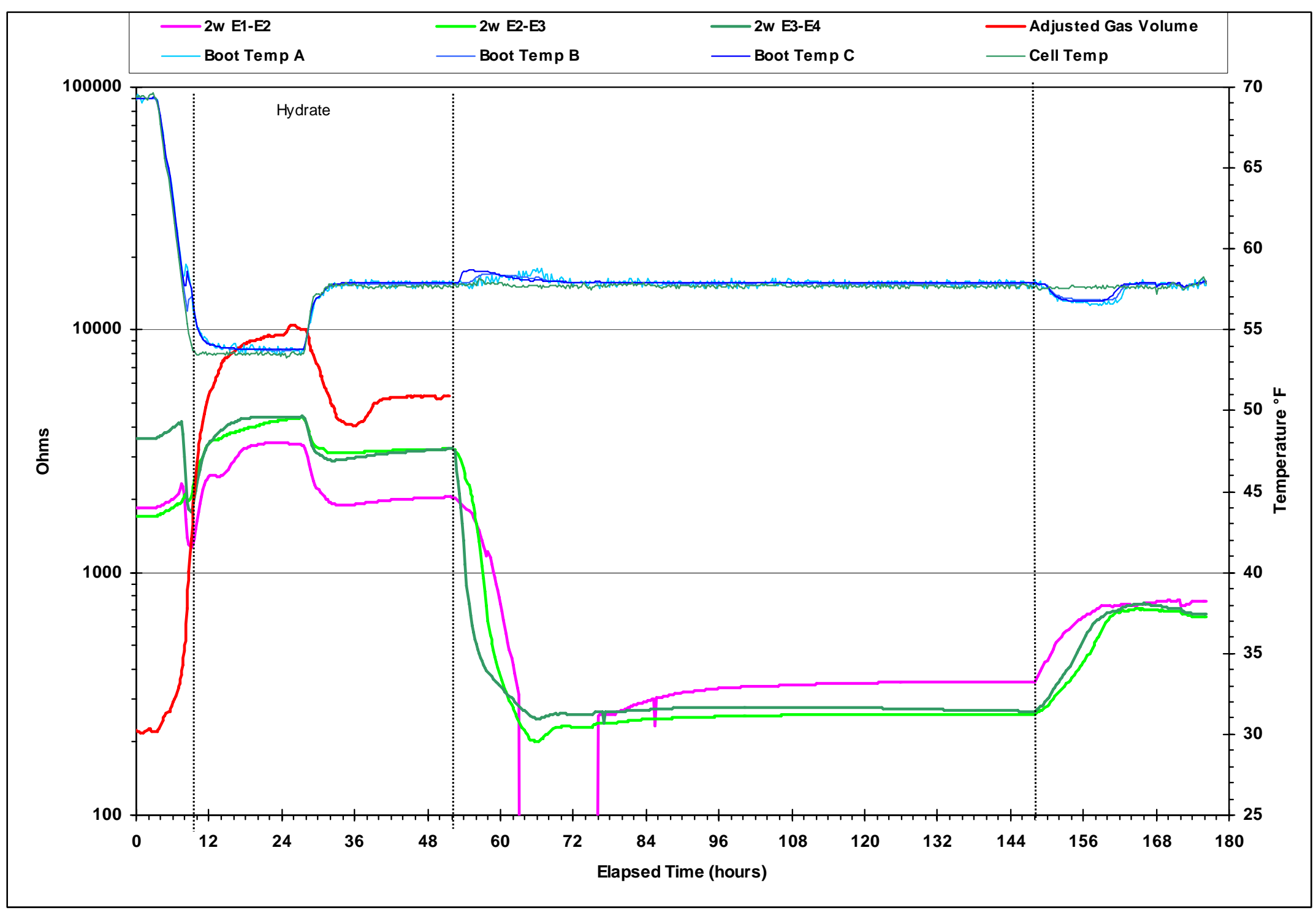


Figure 3.1.30. Test 7: Acoustic Delay Time versus Elapsed Time

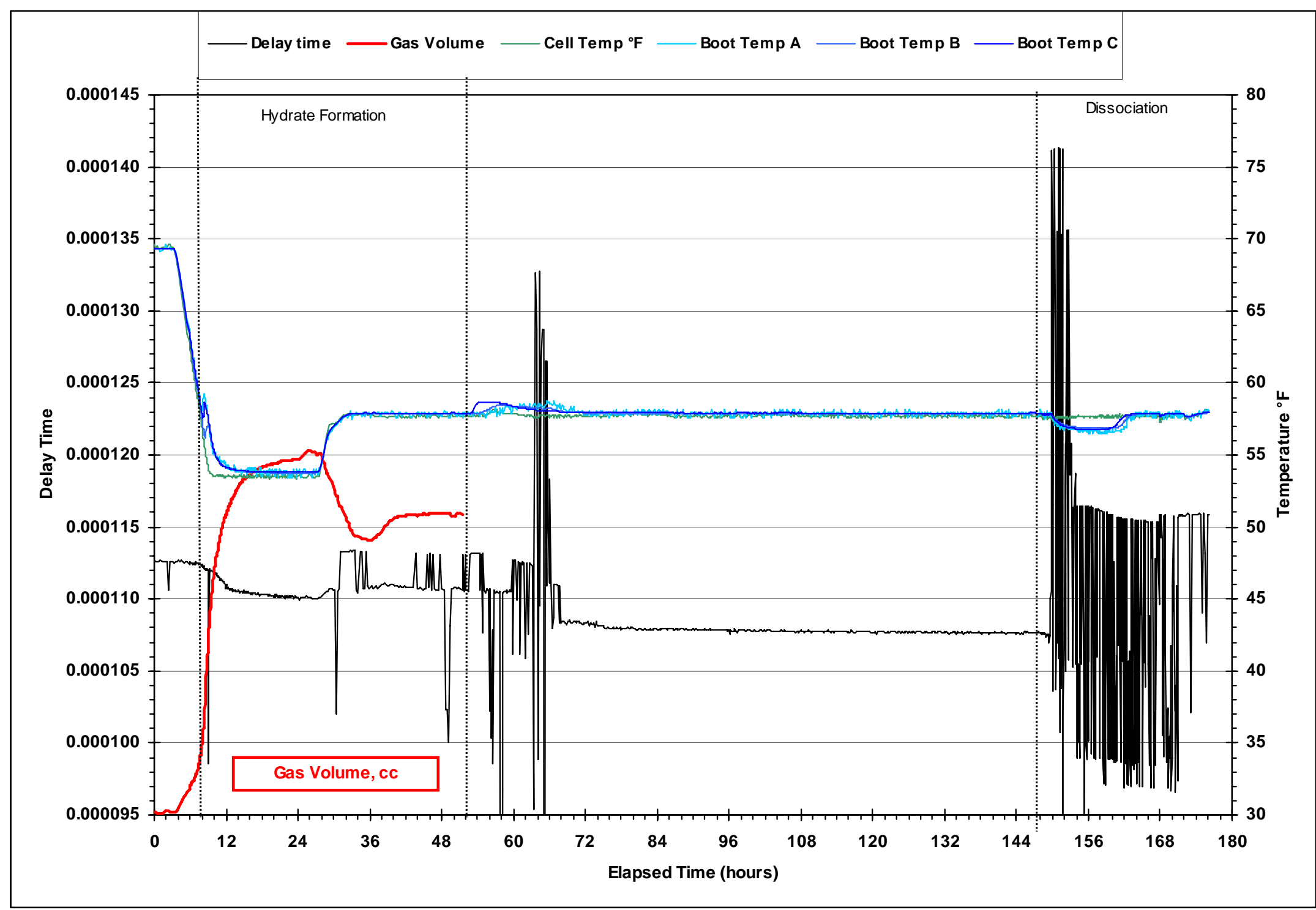


Figure 3.1.31. Test 7: CT Scan Results After Hydrate Formation
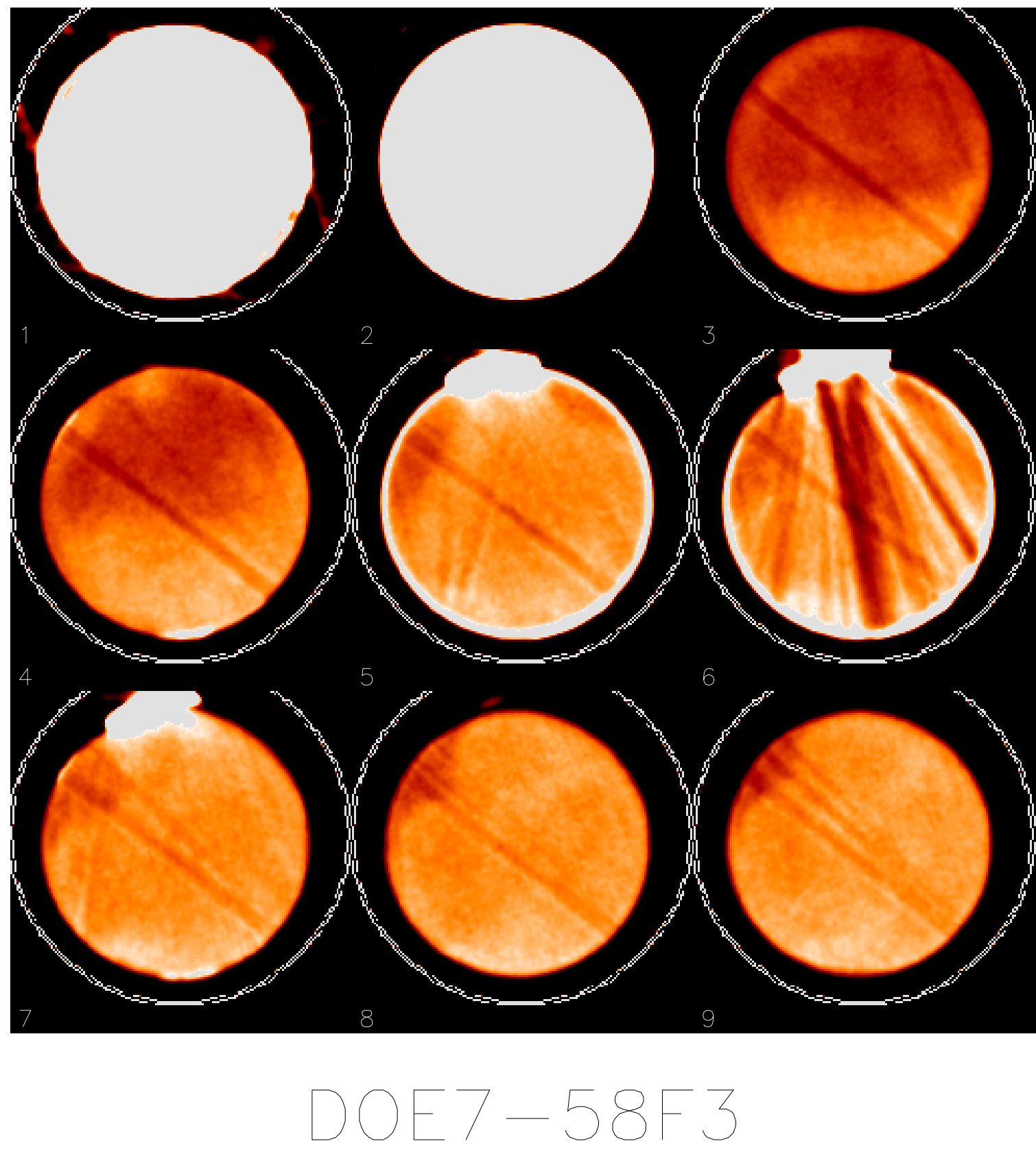

$6 \mathrm{~mm}$ slice spacing

$5 \mathrm{~mm}$ slice thickness 


\subsubsection{Hydrate Formation in QF10 Medium Permeability Core (Test 8)}

Core Material:

Permeability (md):

Hydrate Formation Procedure:

Hydrate Dissociation Procedure:
QF10B

29,000

Constant methane $\mathrm{P}$, decreasing $\mathrm{T}$

No dissociation, power failure

Test 8 was a second repetition of Test 5 . A clean and dry 11.96 inch $(30.38 \mathrm{~cm})$ long by 1.5 inch diameter piece of QF-10 core material was mounted in the jacket and assembled in the core holder with the acoustical sensors, resistivity electrodes and RTD temperature sensors. A net confining stress (NCS) of 500 psi was applied to the core. The core was saturated with $6 \% \mathrm{Nal}$ brine. The pore volume of the core was $133 \mathrm{cc}$ and the porosity was $38 \%$ of pore volume. CT scans were made of the core in the dry and brine saturated conditions. The core was de-saturated with methane gas to $34 \%$ water saturation, leaving $67 \mathrm{cc}$ of gas in the core. The pore pressure was raised to 2,500 psi with methane while simultaneously raising the overburden pressure to $3,000 \mathrm{psi}$, maintaining a constant $500 \mathrm{psi}$ NCS. Once at pressure, the system was leak tested for 3 days prior to the start of the test. All of these operations were carried out at room temperature.

A constant brine pressure test to form hydrate in the QF-10 core was begun. The cell was first cooled to $50^{\circ} \mathrm{F}$ at the rate of $2^{\circ} \mathrm{F} / \mathrm{hr}$. and allowed to equilibrate at that temperature for 3 hours. The cell was then cooled to $46^{\circ} \mathrm{F}$ at the rate of $2^{\circ} \mathrm{F} / \mathrm{hr}$. and allowed to equilibrate for 8 hours. The temperature was then increased to $55^{\circ} \mathrm{F}$. Figure 3.1 .32 shows the temperatures, pump volume of gas injected and pressures for the entire test. Hydrate formation began at $56^{\circ} \mathrm{F}$. Like the Test 5 , the core temperature measured by the RTDs embedded in the jacket showed a sharp increase at the onset of hydrate formation. At the onset of hydrate formation as indicated by the RTDs, the pump began rapidly injecting gas (pump volume increasing) and the pore pressure began declining.

At the onset of hydrate formation, the resistance between the E1 and E2 electrodes sharply increased while the E2-E3 resistance began a slower increase (Figure 3.1.33). This also suggests that the hydrate formed first near the supply end of the core. The core resistance continued to increase for 2 days after the start of hydrate formation then declined and became stable. After 2 days of hydrate formation, the core pressure, resistances and the pump volume had become stable. Sodium Chloride brine was injected into the core to determine relative permeability to brine. High injection pressures were observed, therefore brine injection was stopped.

The test was terminated by a general power failure before dissociation from the core could be attempted. The acoustics data was not recoverable. A CT scan following hydrate formation is shown in Figure 3.1.34. 
Figure 3.1.32. Test 8: Hydrate Formation at Constant Methane Pressure

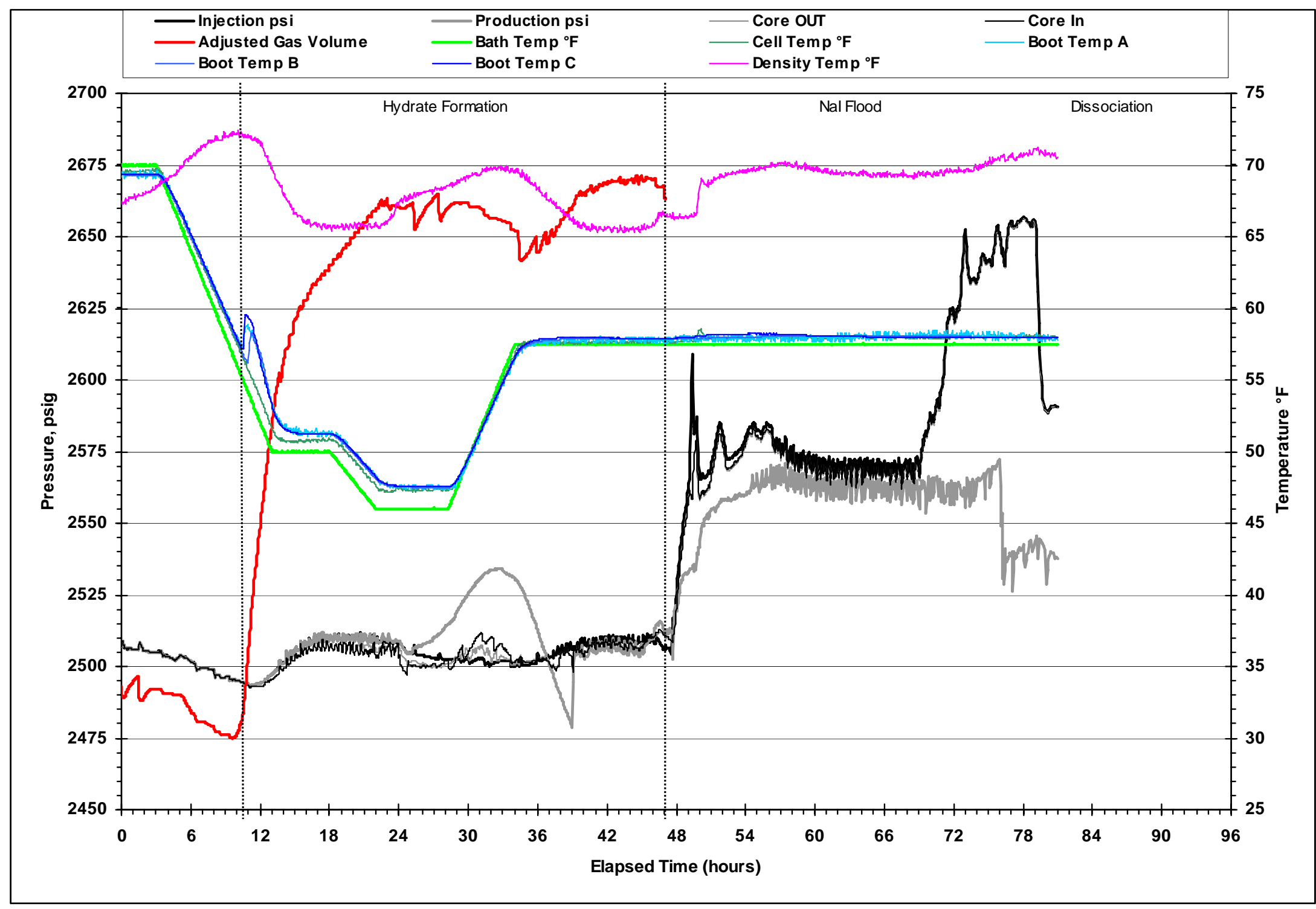


Figure 3.1.33. Test 8: Core Resistivity versus Elapsed Time

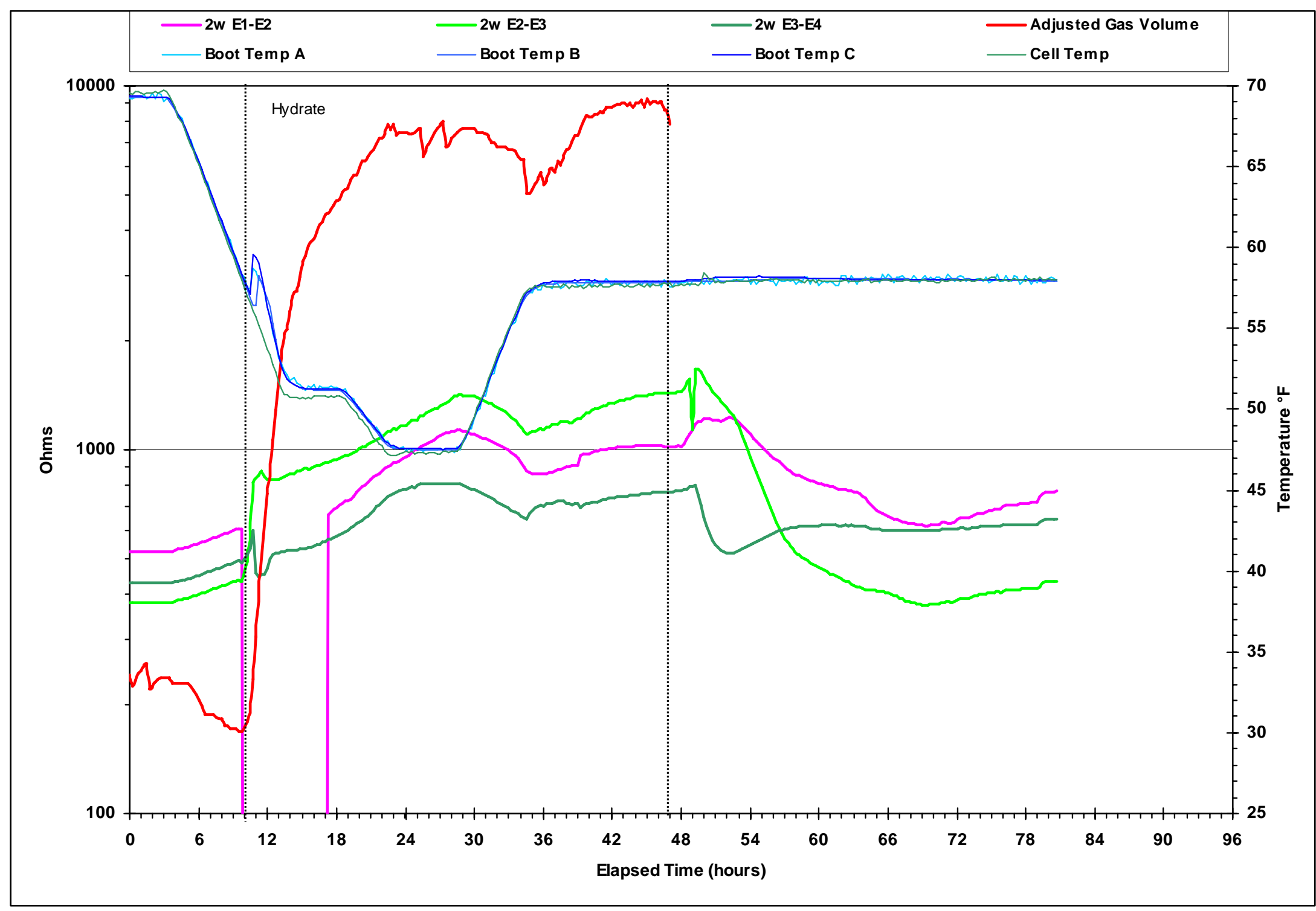


Figure 3.1.34. Test 8: CT Scan Results after Hydrate Formation
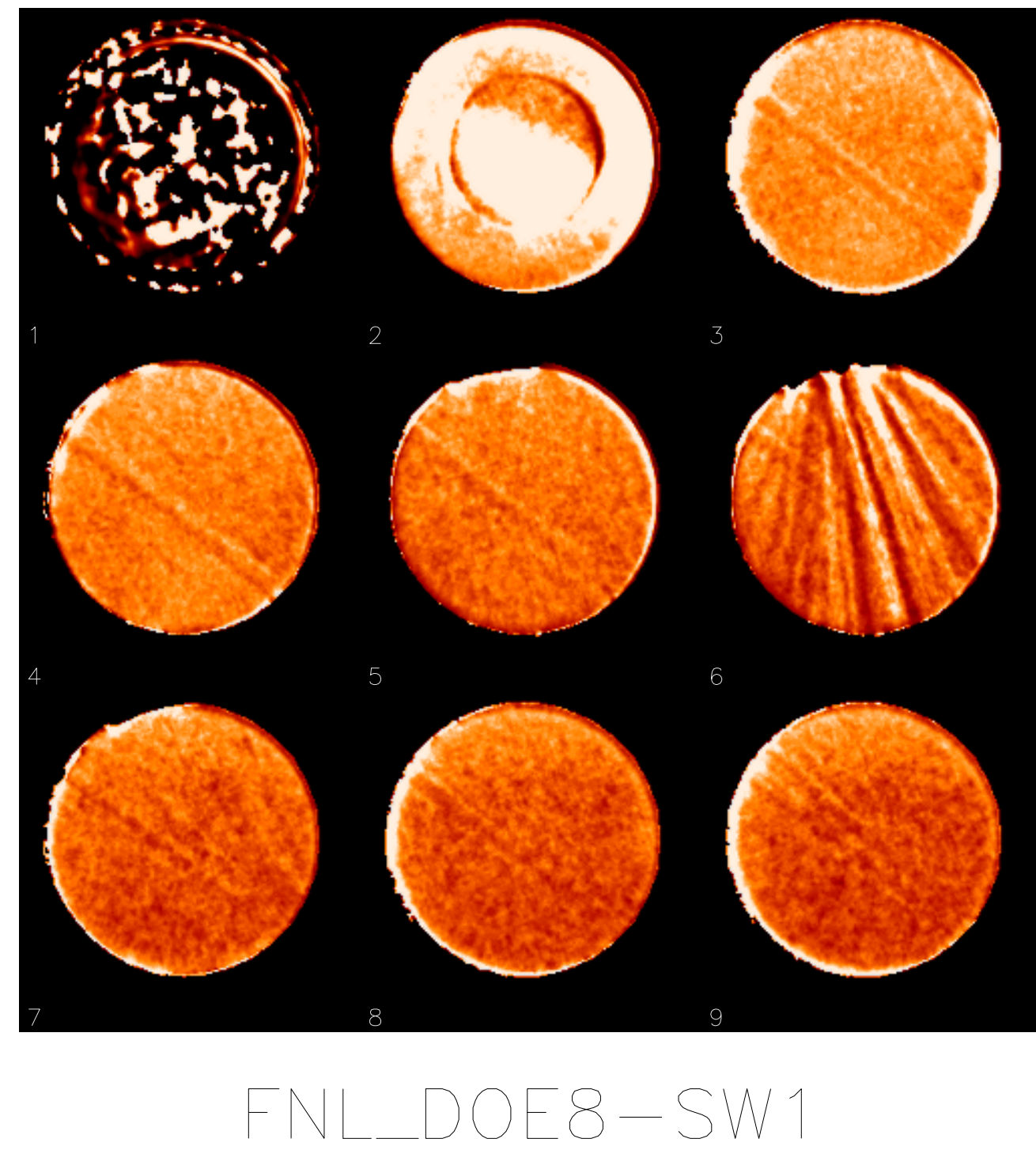

$5 \mathrm{~mm}$ slice spacing

$5 \mathrm{~mm}$ slice thickness

0.0

50.0

100.0 


\subsubsection{Hydrate Formation in QF10 Medium Permeability Core (Test 9)}

Core Material:

QF10B

Permeability (md):

29,000

Hydrate Formation Procedure:

Constant brine $\mathrm{P}$, decreasing $\mathrm{T}$

Hydrate Dissociation Procedure:

No dissociation, power failure

Test 9 was the first experiment using the constant methane mass approach. A clean and dry 11.96 inch $(30.38 \mathrm{~cm}$ ) long by 1.5 inch diameter piece of QF-10 core material was mounted in the jacket and assembled in the core holder with the acoustical sensors, resistivity electrodes and RTD temperature sensors. A net confining stress (NCS) of 500 psi was applied to the core. The core was saturated with $6 \% \mathrm{Nal}$ brine. The pore volume of the core was $133 \mathrm{cc}$ and the porosity was $38 \%$ of pore volume. CT scans were made of the core in the dry and brine saturated conditions. The core was de-saturated with methane gas to $34 \%$ water saturation, leaving $67 \mathrm{cc}$ of gas in the core. The pore pressure was raised to 2,500 psi with methane while simultaneously raising the overburden pressure to 3,000 psi, maintaining a constant 500 psi NCS. Once at pressure, the system was leak tested for 3 days prior to the start of the test. All of these operations were carried out at room temperature.

A constant brine pressure test to form hydrate in the QF-10 core was begun. The cell was first cooled to $56^{\circ} \mathrm{F}$ at the rate of $2^{\circ} \mathrm{F} / \mathrm{hr}$. and allowed to equilibrate at that temperature for 3 day. Figure 3.1 .35 shows the temperatures, pump volume of gas injected and pressures for the entire test.

Hydrate formation began at $56^{\circ} \mathrm{F}$. Like the previous test, the core temperature measured by the RTDs embedded in the jacket showed a sharp increase at the onset of hydrate formation. At the onset of hydrate formation as indicated by the RTDs, the pump began rapidly injecting gas (pump volume increasing) and the pore pressure began declining.

At the onset of hydrate formation, the resistance between the E1 and E2 electrodes sharply increased while the E2-E3 resistance began a slower increase (Figure 3.1.36). This also suggests that the hydrate formed first near the supply end of the core. The core resistance continued to increase for 2 days after the start of hydrate formation then declined and became stable.

The test was terminated by a general power failure before dissociation from the core could be attempted. The acoustics data was not recoverable. A CT scan following hydrate formation is shown in Figure 3.1.37 
Figure 3.1.35. Test 9: Hydrate Formation with Constant Methane Mass

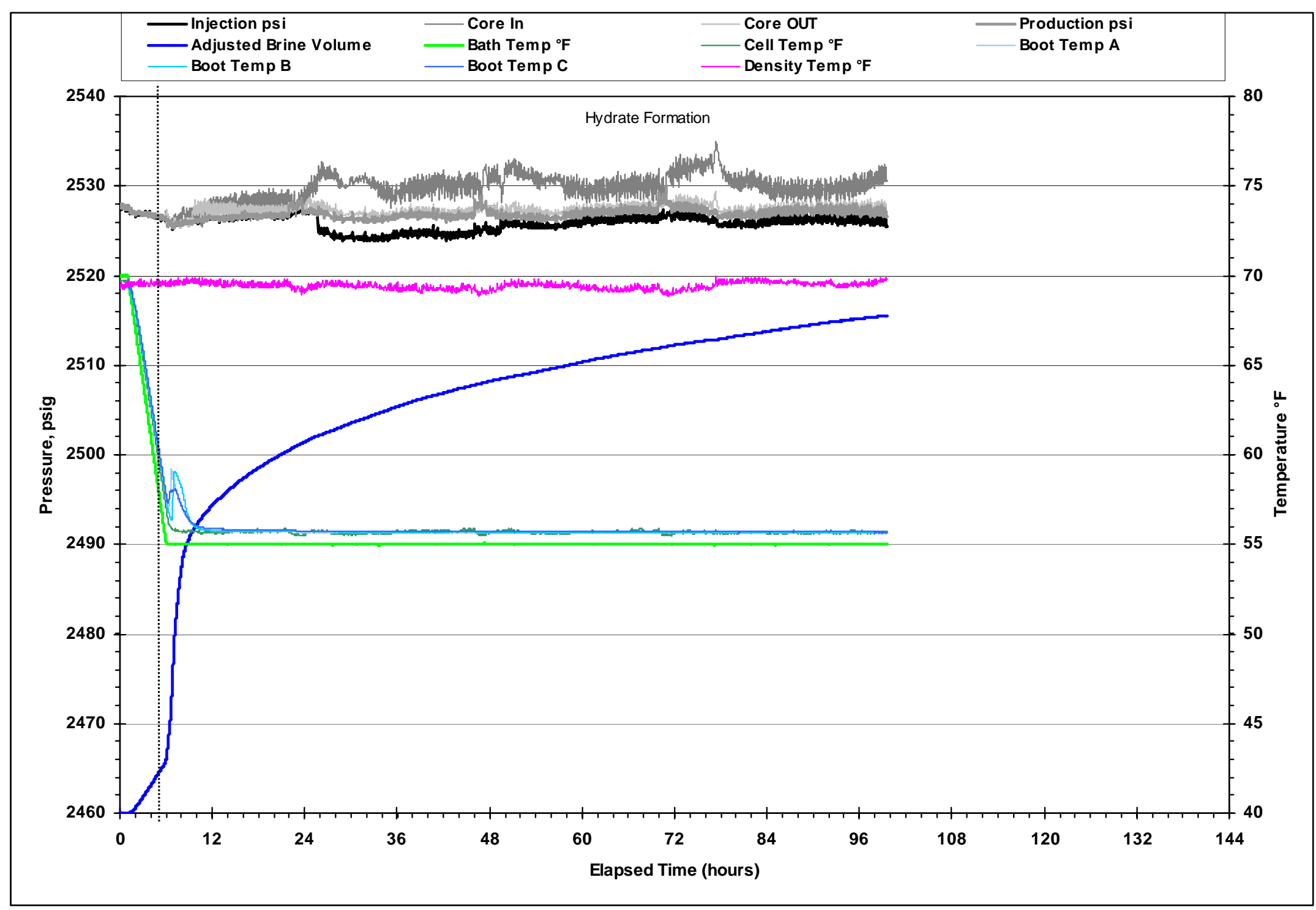

Figure 3.1.36. Test 9: Core Resistance versus Elapsed Time 


\section{INTERTEK WESTPORT TECHNOLOGY CENTER}

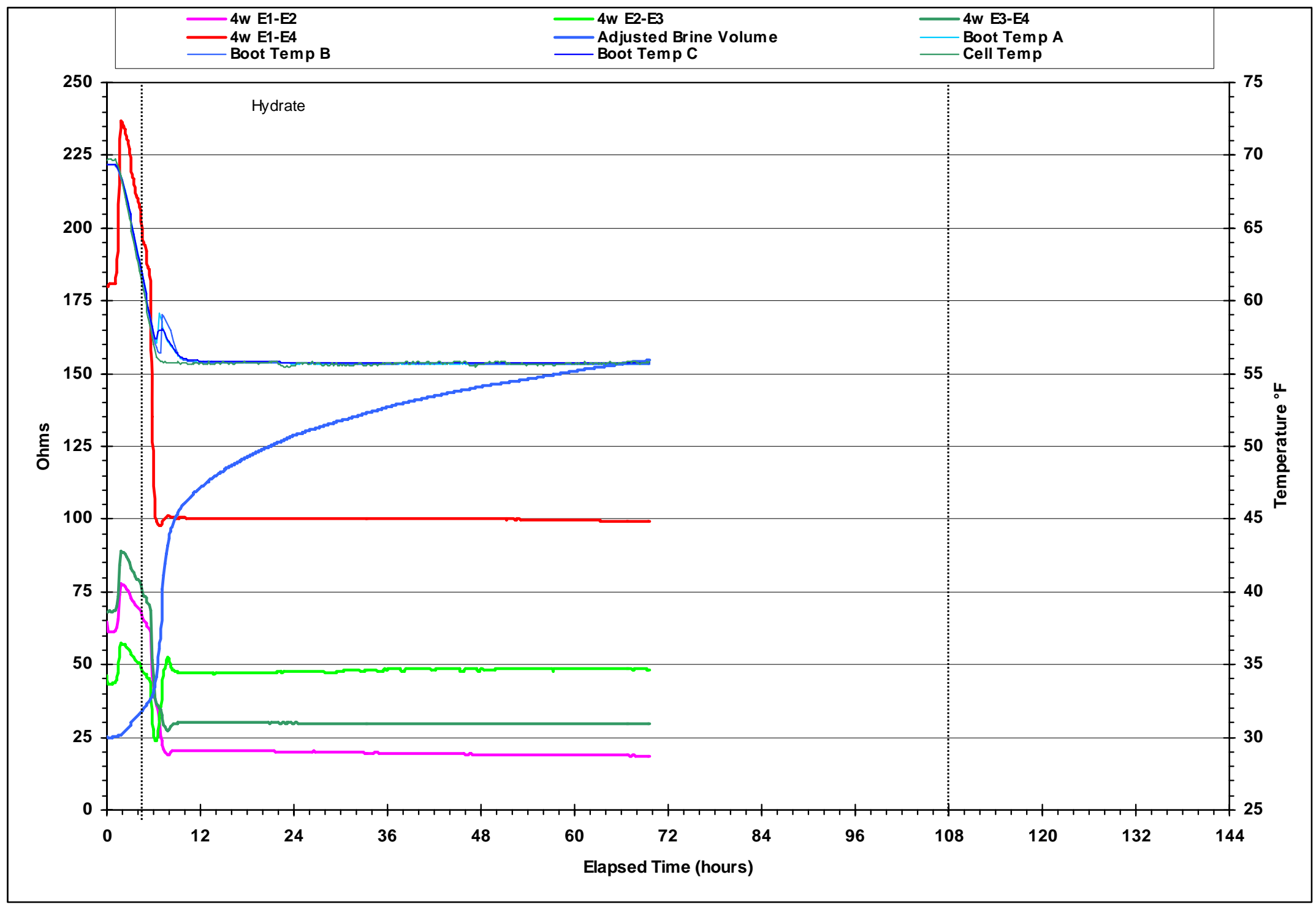


Figure 3.1.37. Test 9: CT Scan After Hydrate Formation

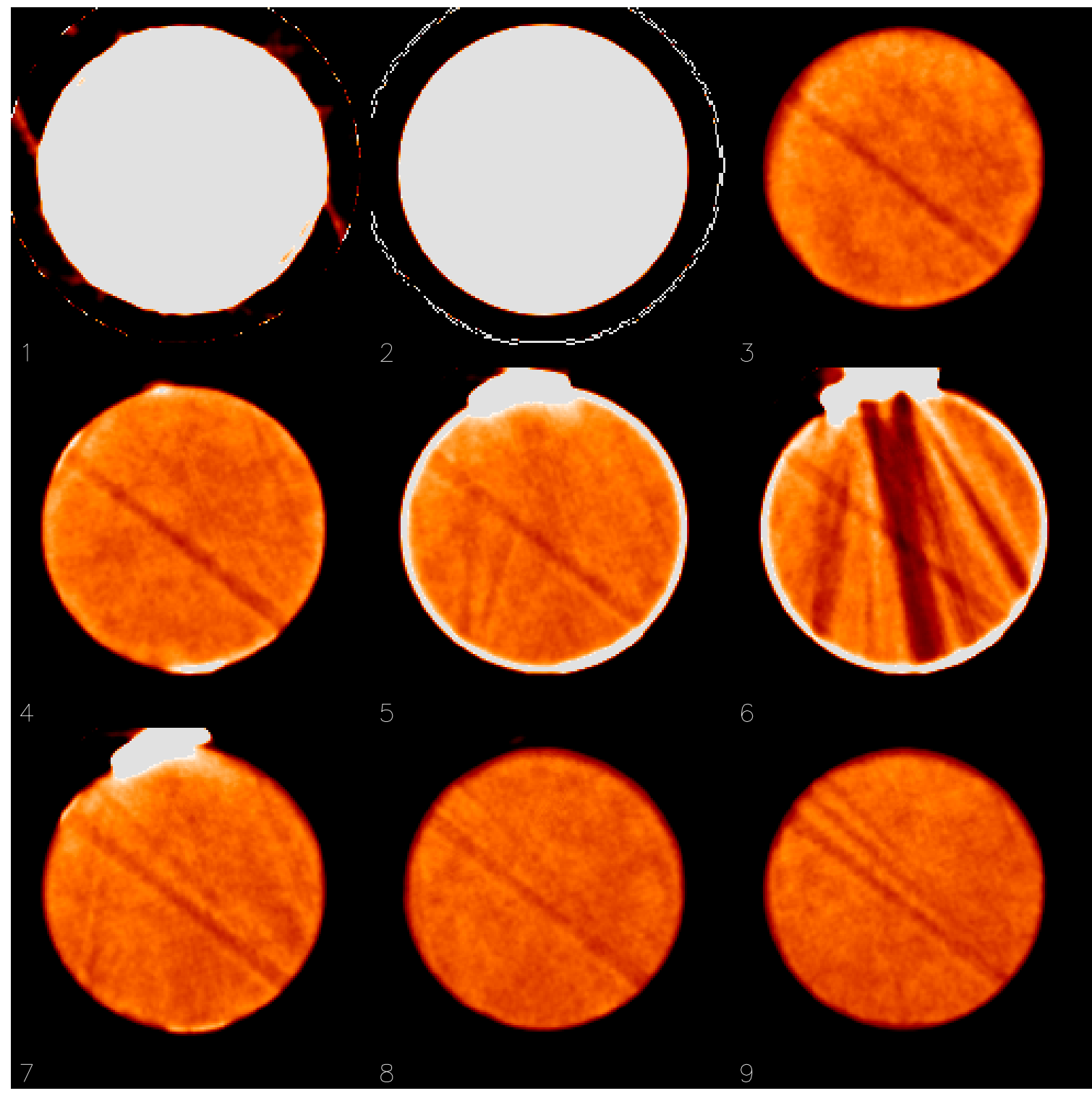

\section{DOE9-7OF2}

$6 \mathrm{~mm}$ slice spacing

$5 \mathrm{~mm}$ slice thickness

700.0

1200.0

1700.0 


\subsubsection{Hydrate Formation in QF10 Medium Permeability Core (Test 10)}

Core Material:

QF10B

Permeability (md):

29,000

Hydrate Formation Procedure:

Constant brine $\mathrm{P}$, decreasing $\mathrm{T}$

Hydrate Dissociation Procedure:

Pressure Depletion

Test 10 was a repetition to replace failed Test 9 . A clean and dry 11.96 inch $(30.38 \mathrm{~cm})$ long by 1.5 inch diameter piece of QF-10 core material was mounted in the jacket and assembled in the core holder with the acoustical sensors, resistivity electrodes and RTD temperature sensors. A net confining stress (NCS) of 500 psi was applied to the core. The core was saturated with $6 \%$ Nal brine. The pore volume of the core was $133 \mathrm{cc}$ and the porosity was $38 \%$ of pore volume. CT scans were made of the core in the dry and brine saturated conditions. The core was de-saturated with methane gas to $34 \%$ water saturation, leaving $67 \mathrm{cc}$ of gas in the core. The pore pressure was raised to 2,500 psi with methane while simultaneously raising the overburden pressure to $3,000 \mathrm{psi}$, maintaining a constant $500 \mathrm{psi}$ NCS. Once at pressure, the system was leak tested for 3 days prior to the start of the test. All of these operations were carried out at room temperature.

A constant brine pressure test to form hydrate in the QF-10 core was begun. The cell was first cooled to $56^{\circ} \mathrm{F}$ at the rate of $2^{\circ} \mathrm{F} / \mathrm{hr}$. and allowed to equilibrate at that temperature for 3 days. Brine was injected into the core to maintain pressure. Previously methane had been injected.

Figure 3.1.29 shows the pressure, temperature and brine injection in Test 10 . Time $t=0$ corresponds to the condition when the core was saturated with gas and water at 2,500 psi and $70^{\circ} \mathrm{F}$ and the bath temperature was changed to $55^{\circ} \mathrm{F}$. Core temperature was decreased to about $55^{\circ} \mathrm{F}$ in 6 hours; and the fluid volume decreased slightly. About $3 \mathrm{ml}$ of brine was injected to maintain the pressure at 2,500 psi. At this time hydrate started forming. The volume decreased indicating the onset of hydrate formation. The shrinkage was compensated by the brine pump, which increased sharply at 6 hours. By about 2 hours, the hydrate formation and hence brine injection decreased. At about 120 hours (5 days), brine injection rate and hence hydration formation, were very slow. About $25 \mathrm{ml}$ of brine was pumped from the beginning of hydrate formation. This would indicate that about $31.3 \mathrm{ml}$ of hydrate was formed. The temperature of boots $A$ and $B$ indicated a local peak between 6 and 12 hours.

At 120 hours, the hydrate formation procedure was stopped and dissociation procedure started. The piston pump was withdrawn to decrease then pressure. Water flowed out at the start of the withdrawal. The pressure is shown in Figure 3.1.38 and the piston withdrawal and the density of the effluent are shown in Figure 3.1.39. The depletion experiment was completed at 170 hours. The final pressure was $625 \mathrm{psi}$; and the produced volume (brine and gas) was $282 \mathrm{ml}$. 
Figure 3.1.40 shows the cross-sectional porosity and saturation distributions estimated from the CT scans. 3D data was collected and averaged to get porosity or saturation as a function of axial location. The average porosity was $36 \%$ in this core, with a total pore volume of $125.2 \mathrm{ml}$. Porosity was almost uniform along the length of the core. Before hydrate formation $\left(2,500 \mathrm{psi}, 55^{\circ} \mathrm{F}\right)$, average gas saturation was $35 \%$ (43.8 $\mathrm{ml})$. The initial gas saturation varied axially, but was a little higher at the production end of the core. After hydrate formation, another scan was taken. The average hydrate saturation was $20.4 \%$ $(25.6 \mathrm{ml})$, and the rest was brine. Hydrate saturation varied axially, like the initial gas saturation. After dissociation, a scan was taken at about 139 hours. The average gas saturation was $54.6 \%$ (68.4 ml), and rest was brine. The gas saturation was fairly uniform except at the production end.

Figure 3.1.41 shows the electrical resistance between 4 electrodes placed along the length of the core. As the brine was injected before hydrate formation, electrical resistance decreased. As brine was taken out during pressure depletion, electrical resistance increased. Figure 3.1.42 shows the acoustic delay time in Test 10.

Figure 3.1.43 shows the CT scan images after hydrate formation during Test 10. The darker colors indicate areas of lower densities (lower CT numbers), and the lighter colors indicate areas of higher densities (higher CT numbers). The red color indicates areas of gas saturation. The white color indicates areas of brine saturation. 
Figure 3.1.38. Test 10: Hydrate Formation with Constant Methane Mass

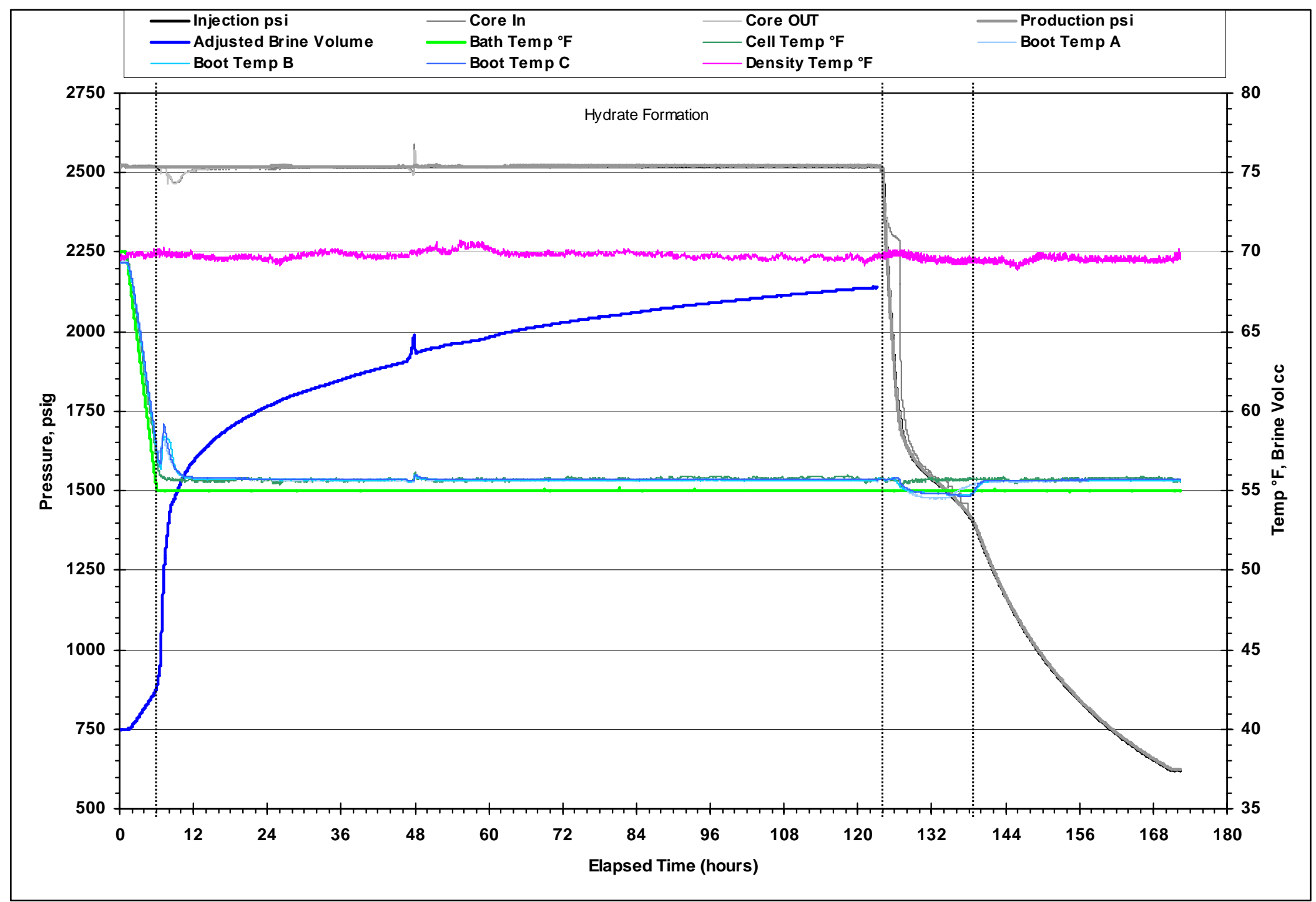


Figure 3.1.39. Test 10: Brine Injection, Depletion Volume and Production Density

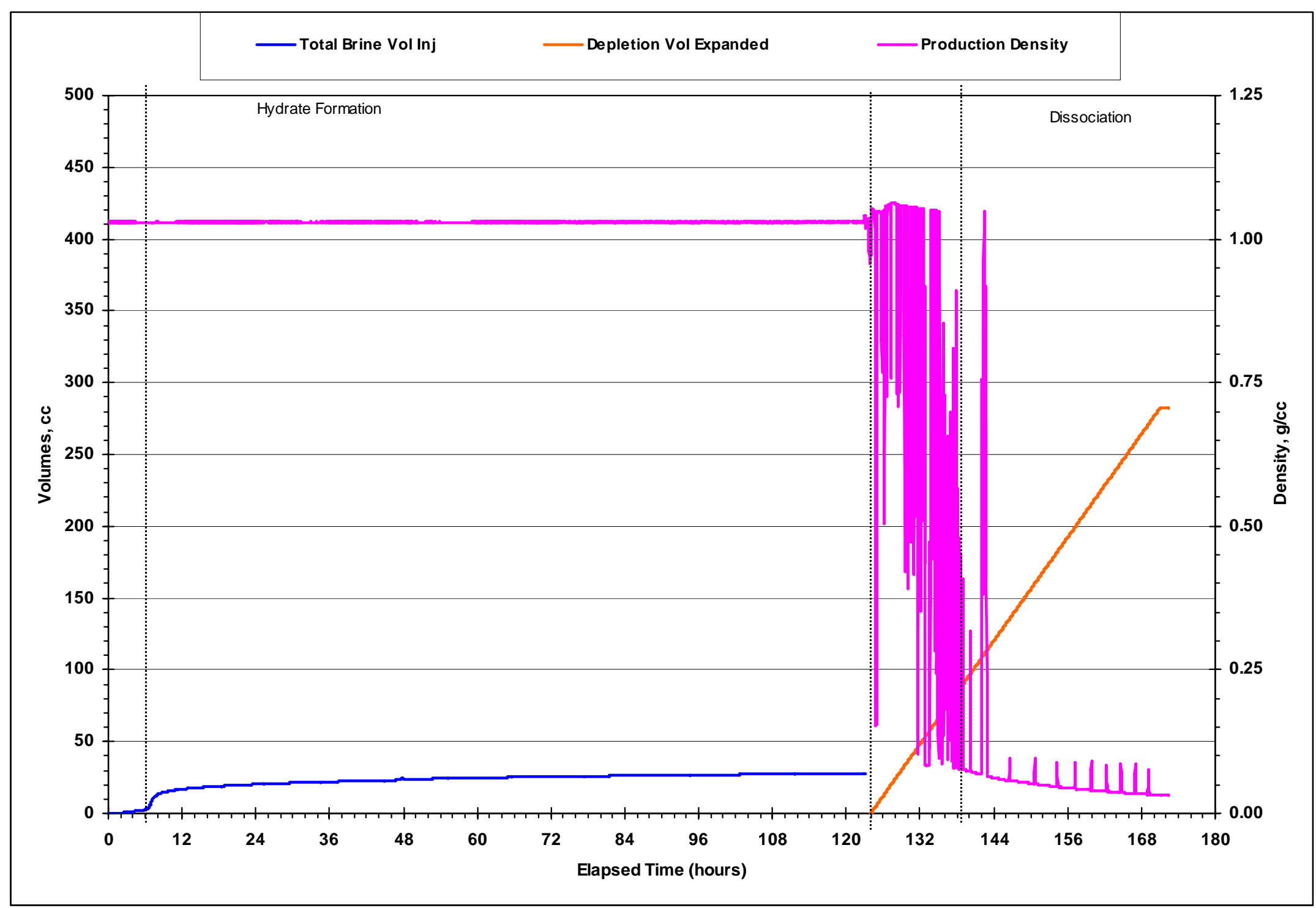


Figure 3.1.40. Test 10: Saturation and Porosity Derived from CT Scans

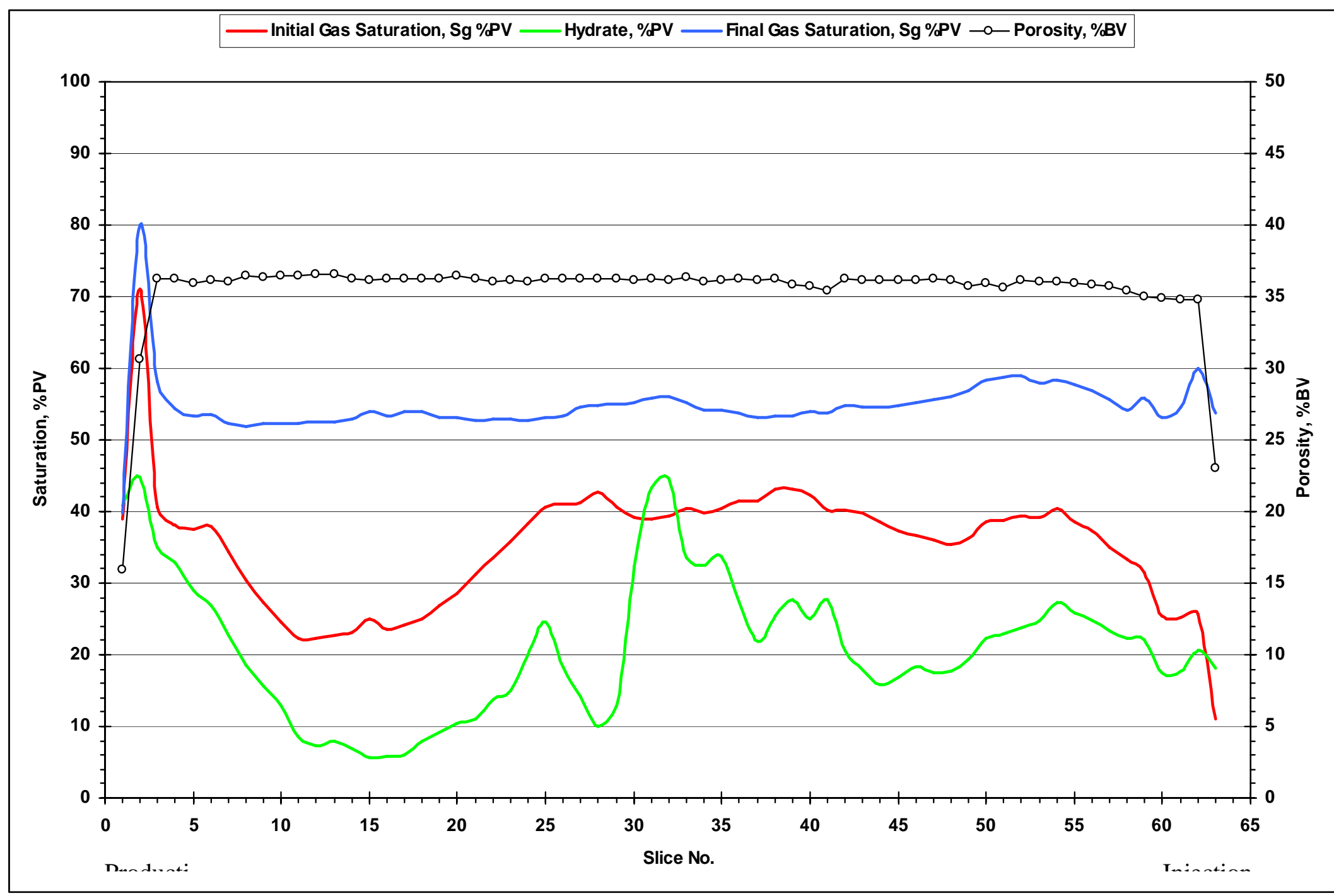


Figure 3.1.41. Test 10: Electrical Resistance, Brine Injection and Depletion Volume

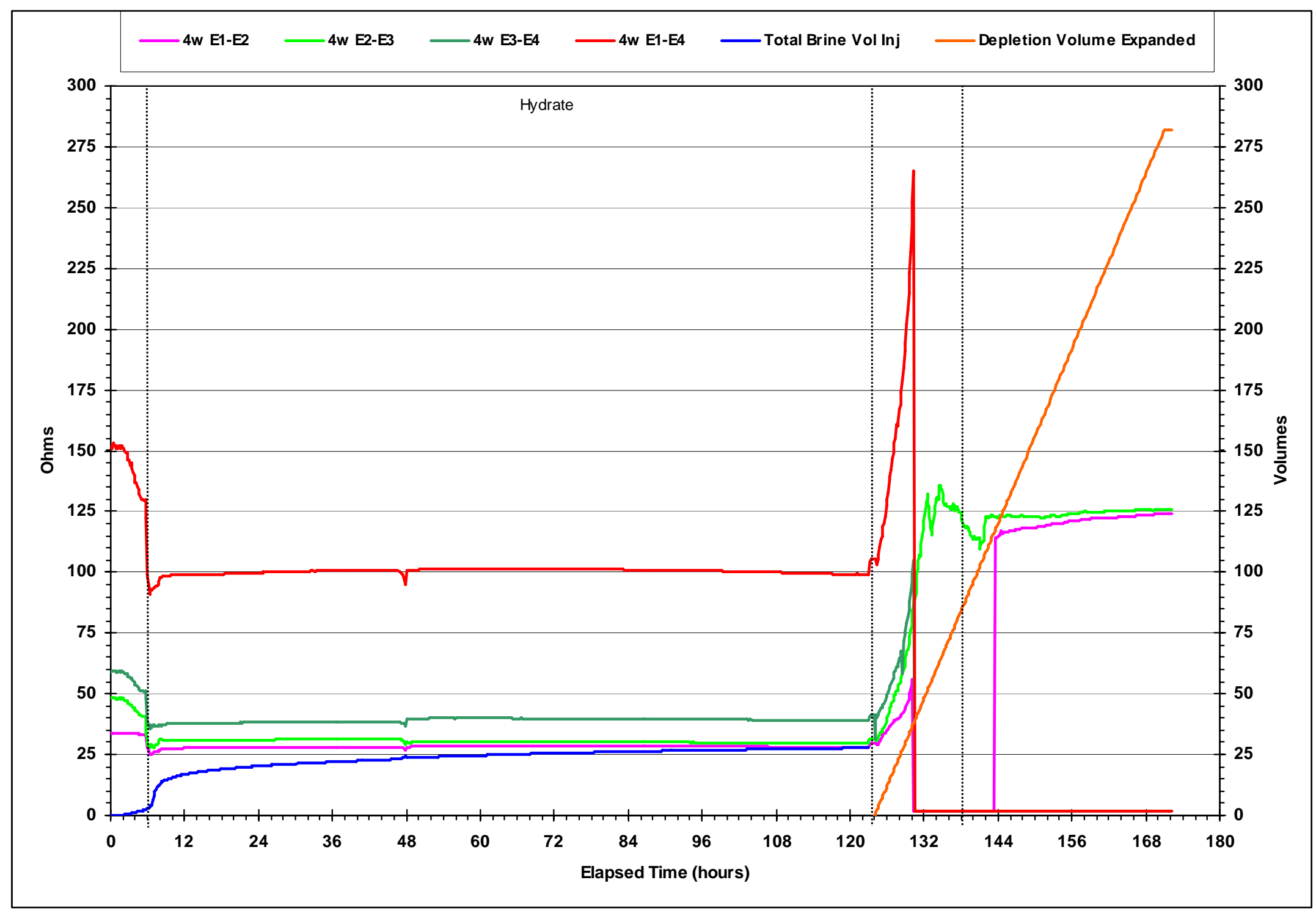


Figure 3.1.42. Test 10: Acoustic Delay Time, Brine Injection and Depletion Volume

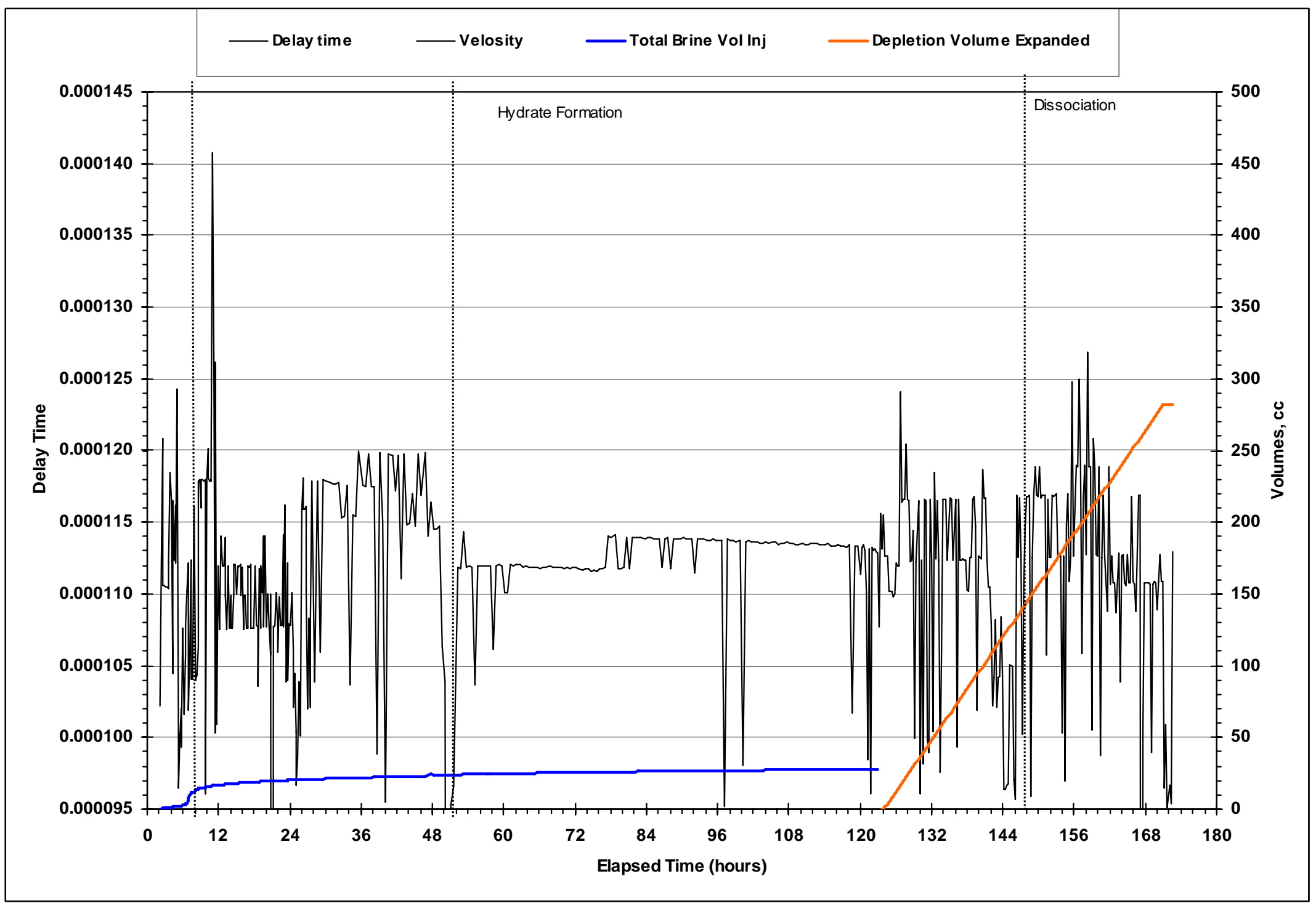


Figure 3.1.43. Test 10 CT Scan Results After Hydrate Formation
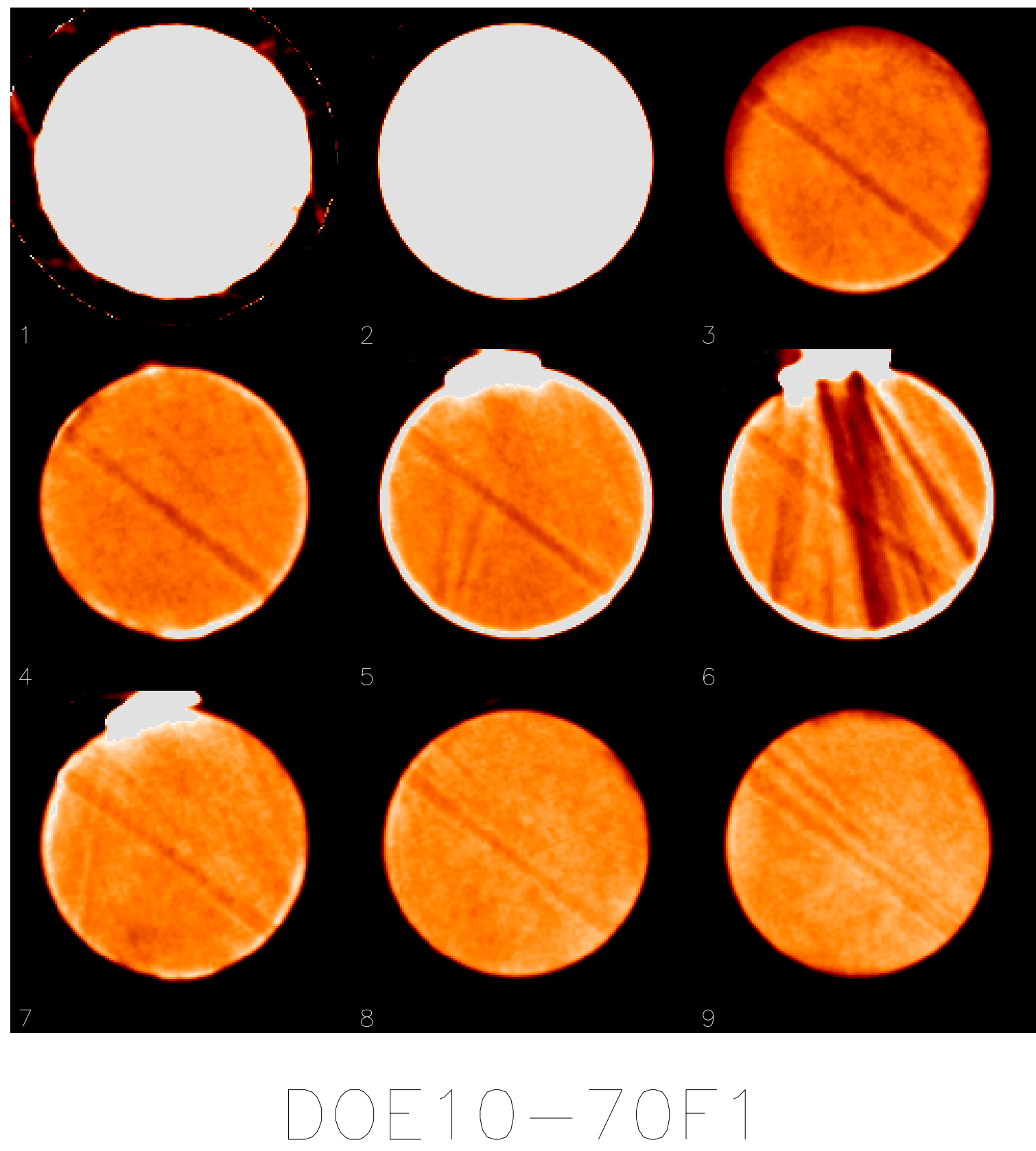

$5 \mathrm{~mm}$ slice thickness 


\subsubsection{Hydrate Formation in QF10 Medium Permeability Core (Test 11)}

Core Material:

Permeability (md):

Hydrate Formation Procedure:

Hydrate Dissociation Procedure:
QF10G

29,000

Constant brine $\mathrm{P}$, decreasing $\mathrm{T}$

Pressure Depletion

Test 11 was the first repetition of Test 10 . A clean and dry 11.87 inch $(30.16 \mathrm{~cm})$ long by 1.5 inch diameter piece of QF-10 core material was mounted in the jacket and assembled in the core holder with the acoustical sensors, resistivity electrodes and RTD temperature sensors. A net confining stress (NCS) of 500 psi was applied to the core. The core was saturated with $6 \% \mathrm{Nal}$ brine. The pore volume of the core was $130 \mathrm{cc}$ and the porosity was $36 \%$ of pore volume. CT scans were made of the core in the dry and brine saturated conditions. The core was de-saturated with methane gas to $34 \%$ water saturation, leaving $67 \mathrm{cc}$ of gas in the core. The pore pressure was raised to 2,500 psi with methane while simultaneously raising the overburden pressure to $3,000 \mathrm{psi}$, maintaining a constant $500 \mathrm{psi}$ NCS. Once at pressure, the system was leak tested for 3 days prior to the start of the test. All of these operations were carried out at room temperature.

A constant brine pressure test to form hydrate in the QF-10 core was begun. The cell was first cooled to $56^{\circ} \mathrm{F}$ at the rate of $2^{\circ} \mathrm{F} / \mathrm{hr}$. and allowed to equilibrate at that temperature for 3 days. Figure 3.1.44 shows the temperatures, pump volume of gas injected and pressures for the entire test. Brine was injected into the core to maintain pressure.

Hydrate formation began at $56^{\circ} \mathrm{F}$. Like the previous test, the core temperature measured by the RTDs embedded in the jacket showed a sharp increase at the onset of hydrate formation. At the onset of hydrate formation as indicated by the RTDs, the pump began rapidly injecting gas (pump volume increasing) and the pore pressure began declining. The brine pump failed at $74 / \mathrm{hr}$. and the pressure fell briefly. Brine injection was resumed.

At $120 \mathrm{hr}$., hydrate formation is stopped and dissociation is started. The piston pump was withdrawn to decrease the pressure. -The pressure fell sharply. As the pressure reached the equilibrium brine-gashydrate pressure, hydrate dissociated and some of the methane gas started flowing out. The withdrawal was $2 \mathrm{ml} / \mathrm{hr}$. The volume and density of the effluent are shown in Figure 3.1.45. The depletion experiment is stopped at 210 hours. The final pressure was $625 \mathrm{psi}$; the produced volume (brine and gas) was about $188 \mathrm{ml}$.

Figure 3.1.46 shows cross-sectional porosity and saturation distributions estimated from CT scans. Before hydrate formation $\left(2,500 \mathrm{psi}, 55^{\circ} \mathrm{F}\right)$, the average gas saturation was $22.5 \%$ (28.3 ml). After hydrate formation, another scan indicated an average hydrate saturation of $11.5 \%(14.4 \mathrm{ml})$ and the rest 
was brine. Hydrate saturation varied axially, like the initial gas saturation. (These numbers are subject to misinterpretation because of salt concentration change in brine. It is also possible that some methane may have been present in the gas form.) After dissociation, the average gas saturation was $45.5 \%$ ( 57.2 $\mathrm{ml}$ ) and the rest was brine.

Figure 3.1.47 shows the electrical resistance between 4 electrodes placed along the length of the core. As the brine was injected before hydrate formation, electrical resistance decreased. At the onset of hydrate formation, the resistance between the E1 and E2 and E2-E3 electrodes decreased a small amount due to the increased brine saturation in the core. The core resistance stabilized for 5 days and increased when dissociation started. As brine was taken out during pressure depletion, electrical resistance increased. (Electrical resistance depends on brine saturation and salinity.)

Figure 3.1.48 shows the acoustic delay time results for Test 11. CT images shown in Figure 3.1.49 show the presence of hydrate in the core. The darker colors indicate areas of lower densities (lower CT numbers), and the lighter colors indicate areas of higher densities (higher CT numbers). The red color indicates areas of gas saturation. The white color indicates areas of brine saturation. 
Figure 3.1.44. Test 11: Hydrate Formation with Constant Methane Mass

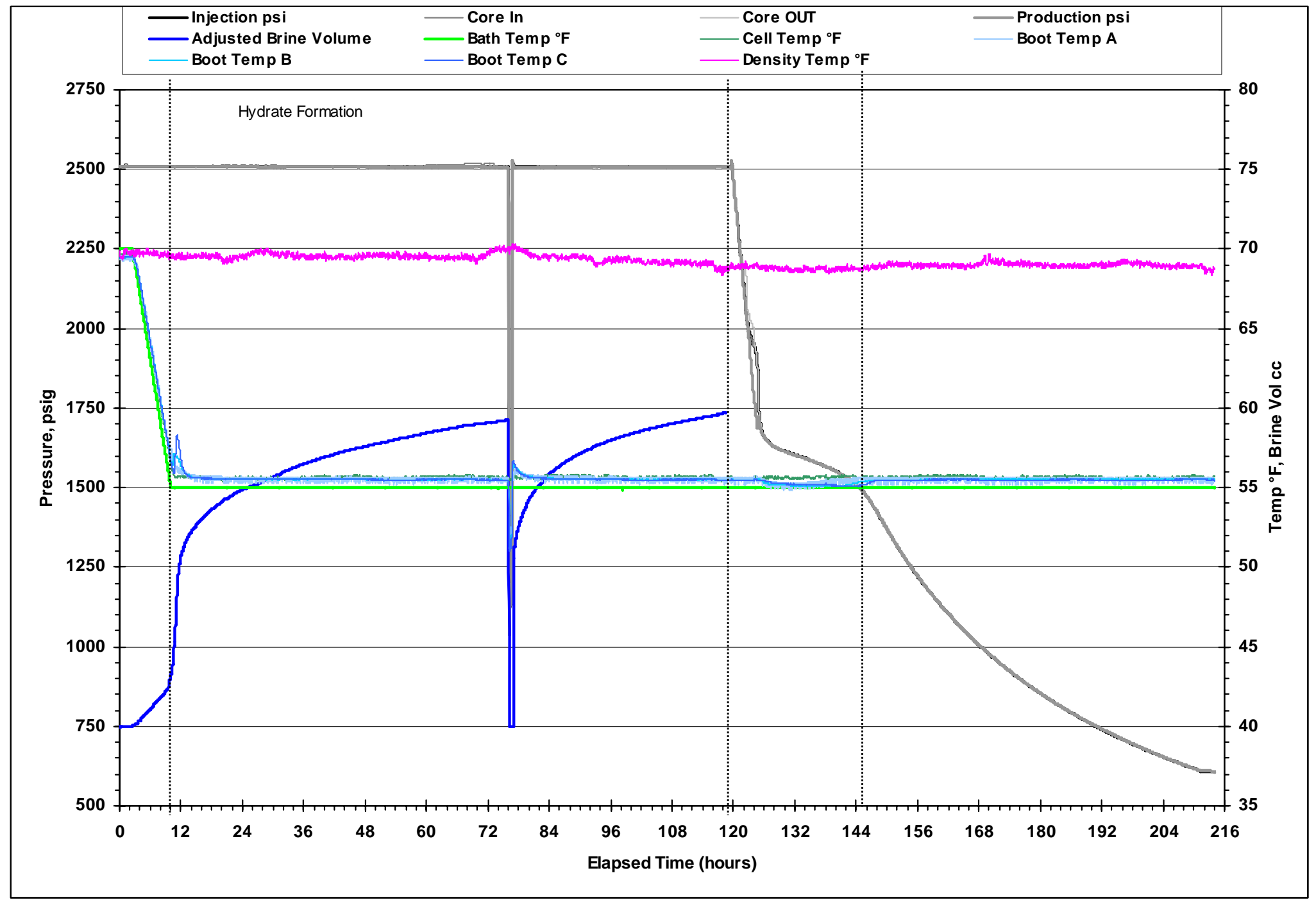


Figure 3.1.45. Test 11: Brine Injection, Depletion Volume and Production Density

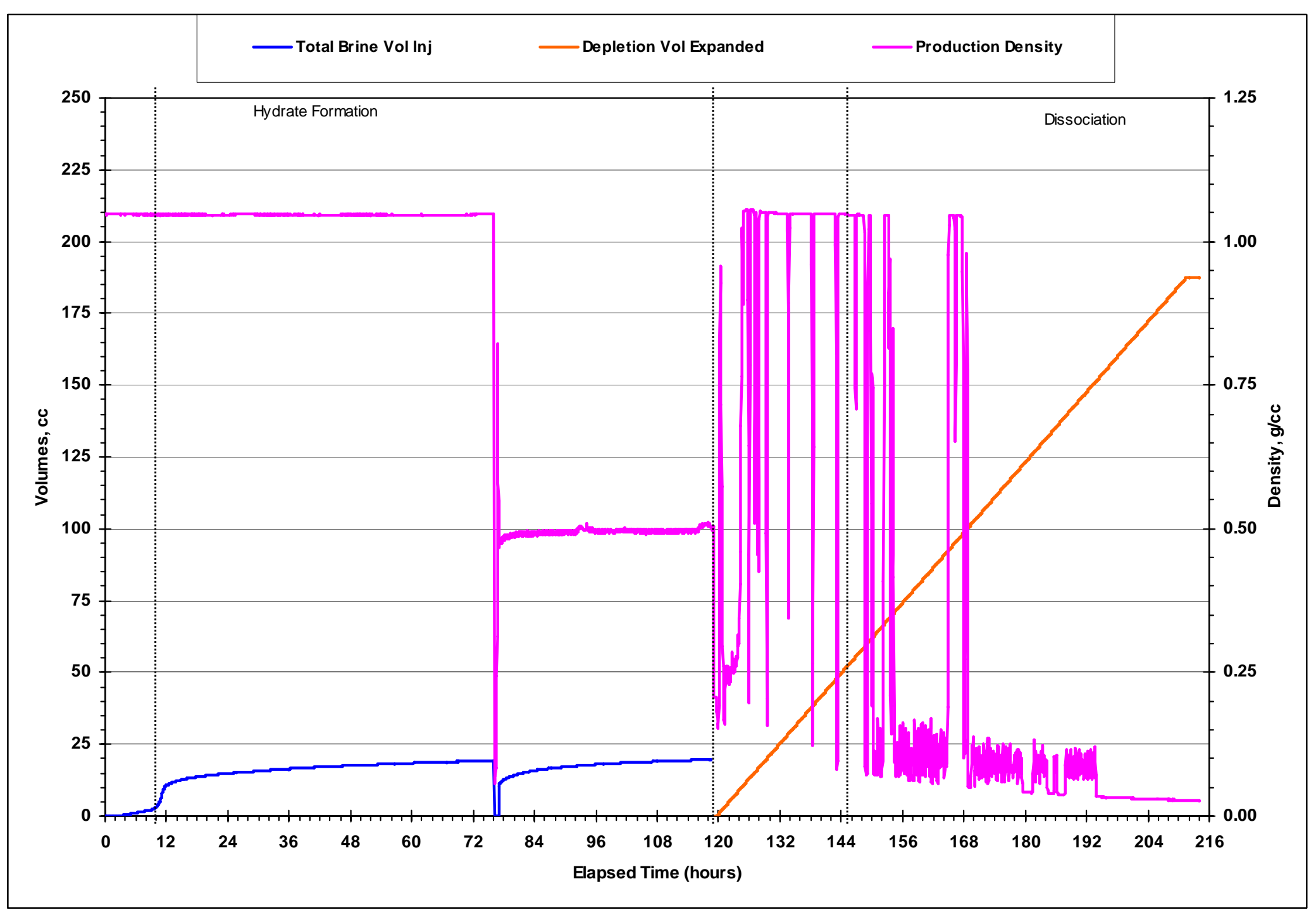


Figure 3.1.46. Test 11: Saturation and Porosity Derived from CT Scans

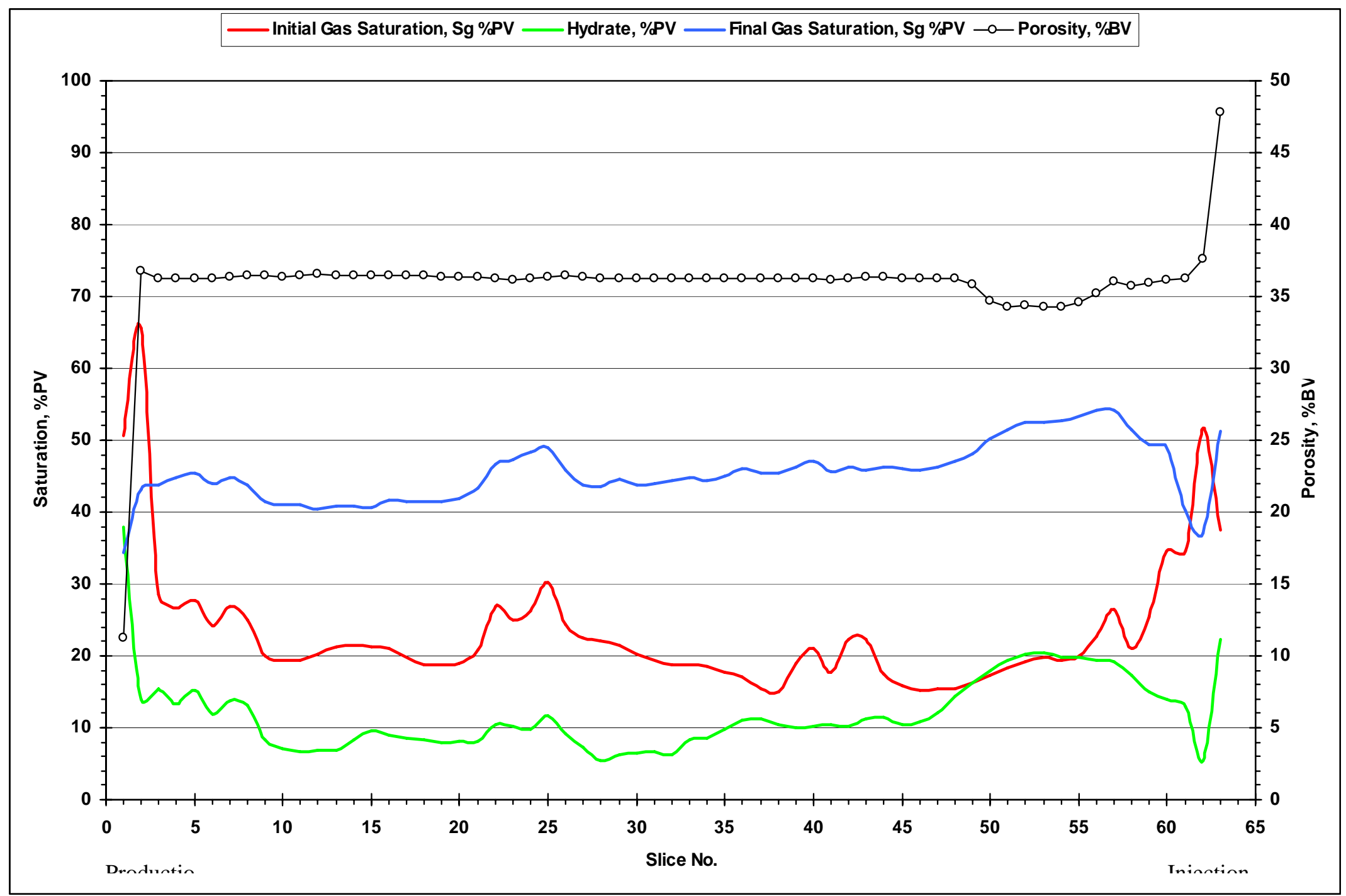


Figure 3.1.47. Test 11: Electrical Resistance, Brine Injection and Depletion Volume

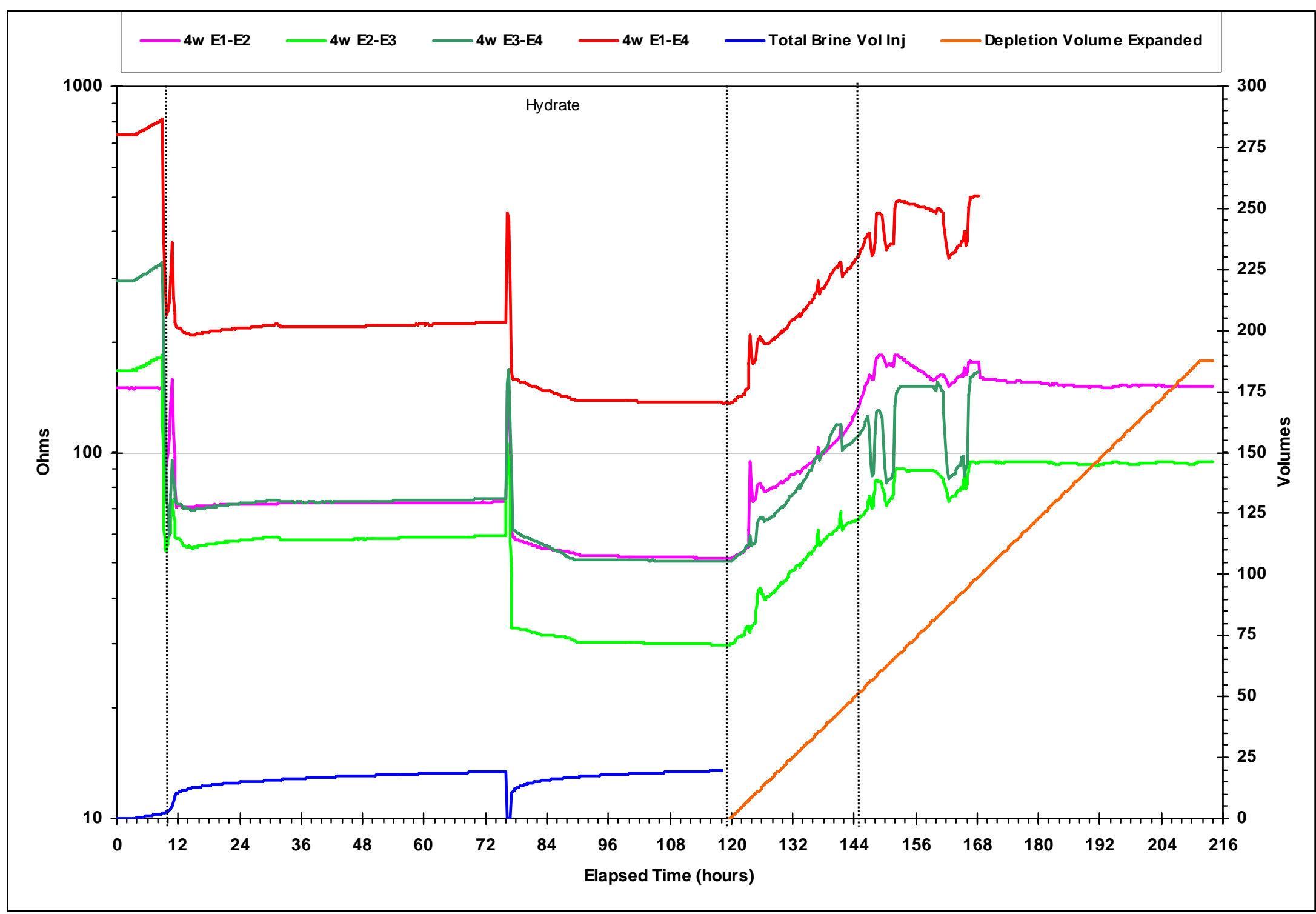


Figure 3.1.48. Test 11: Acoustic Delay Time, Brine Injection and Depletion Volume

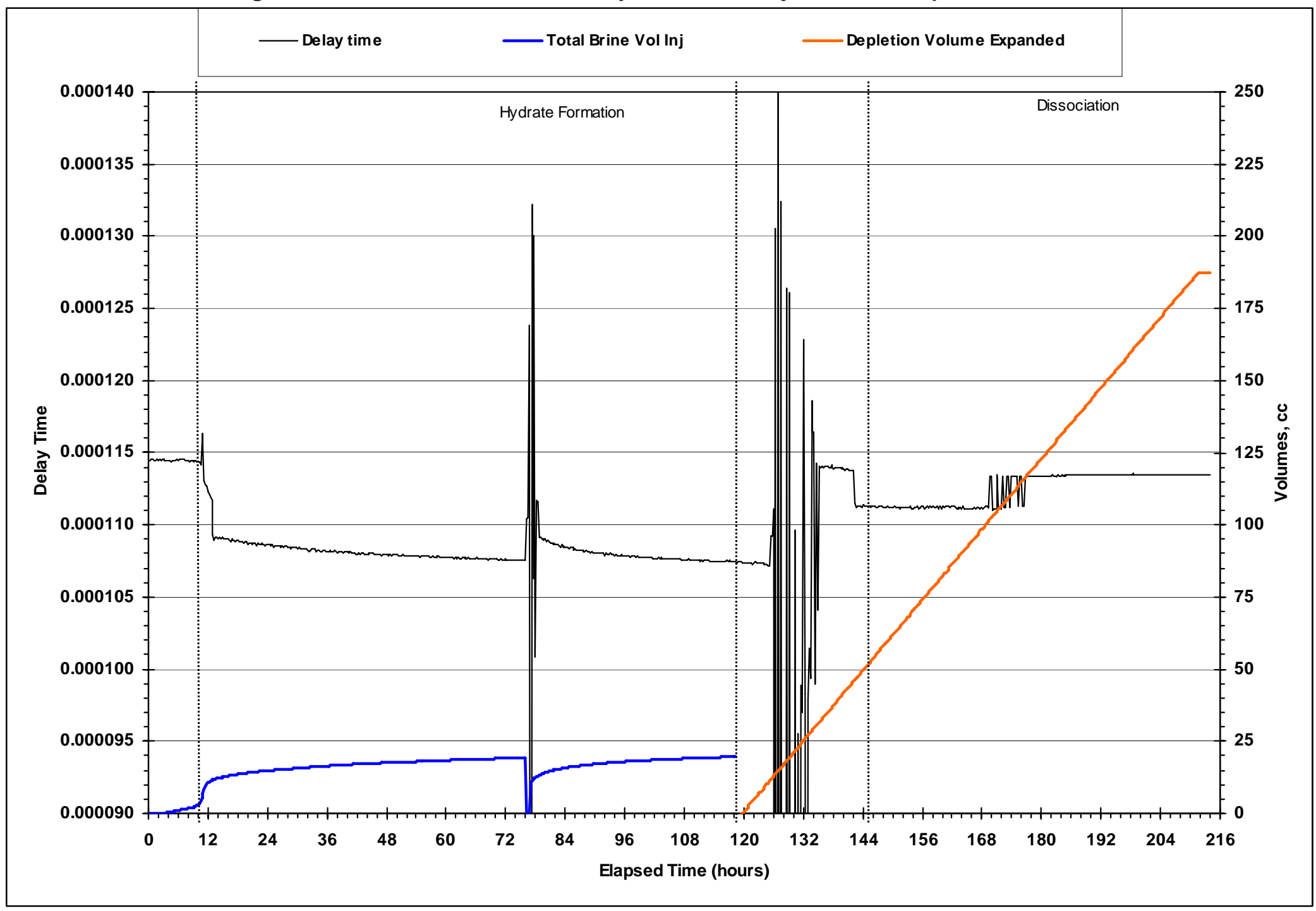


Figure 3.1.49. Test 11: CT Scan Results After Hydrate Formation
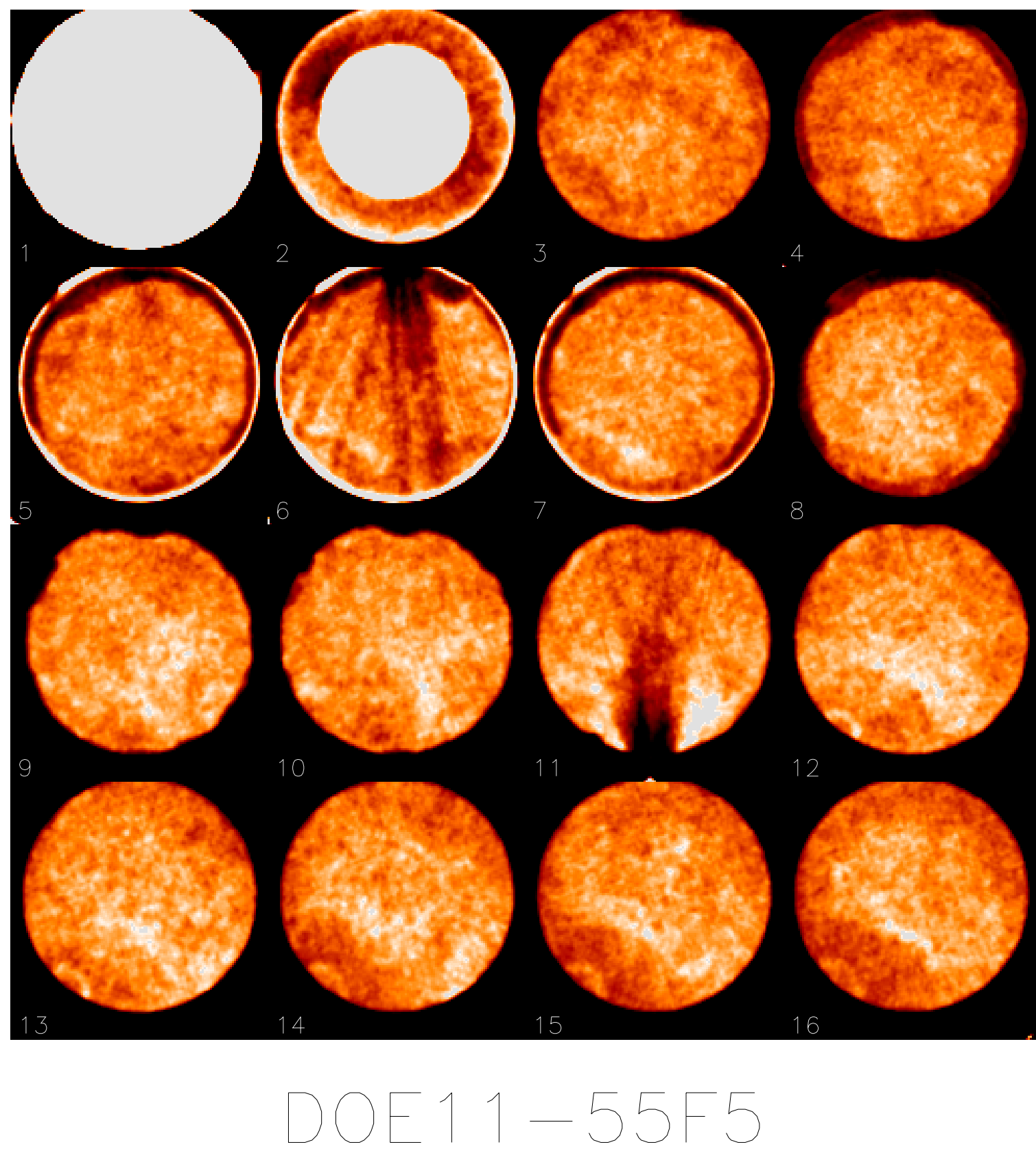

$5 \mathrm{~mm}$ slice spacing

$5 \mathrm{~mm}$ slice thickness

1100.0

1300.0

1500.0 


\subsubsection{Hydrate Formation in QF10 Medium Permeability Core (Test 12)}

Core Material:

Permeability (md):

Hydrate Formation Procedure:

Hydrate Dissociation Procedure:
QF10 G

29,000

Constant brine $\mathrm{P}$, decreasing $\mathrm{T}$

Pressure Depletion

Test 12 was a second repetition of Test 10. A clean and dry 11.87 inch $(30.16 \mathrm{~cm})$ long by 1.5 inch diameter piece of QF-10 core material was mounted in the jacket and assembled in the core holder with the acoustical sensors, resistivity electrodes and RTD temperature sensors. A net confining stress (NCS) of 500 psi was applied to the core. The core was saturated with $6 \% \mathrm{Nal}$ brine. The pore volume of the core was $130 \mathrm{cc}$ and the porosity was $36 \%$ of pore volume. CT scans were made of the core in the dry and brine saturated conditions. The core was de-saturated with methane gas to $34 \%$ water saturation, leaving $67 \mathrm{cc}$ of gas in the core. The pore pressure was raised to 2,500 psi with methane while simultaneously raising the overburden pressure to $3,000 \mathrm{psi}$, maintaining a constant $500 \mathrm{psi}$ NCS. Once at pressure, the system was leak tested for 3 days prior to the start of the test. All of these operations were carried out at room temperature.

A constant brine pressure test to form hydrate in the QF-10 core was begun. The cell was first cooled to $56^{\circ} \mathrm{F}$ at the rate of $2^{\circ} \mathrm{F} / \mathrm{hr}$. and allowed to equilibrate at that temperature for 3 days. Brine was injected into the core to maintain pressure.

Hydrate formation began at $56^{\circ} \mathrm{F}$. Like the previous test, the core temperature measured by the RTDs embedded in the jacket showed a sharp increase at the onset of hydrate formation. At the onset of hydrate formation as indicated by the RTDs, the pump began rapidly injecting gas (pump volume increasing) and the pore pressure began declining.

This experiment was similar to Test 10 except that the pressure depletion rate was $2 \mathrm{ml} / \mathrm{hr}$. At 120/hr., hydrate formation was stopped and dissociation was started. The piston pump was withdrawn to decrease the pressure. The pressure is shown in Figure 3.1.50 and the piston withdrawal and the density of the effluent are shown in Figure 3.1.51. Water flowed out at the start of the withdrawal. The pressure fell sharply. As the pressure reached the equilibrium brine-gas-hydrate pressure, the hydrate dissociated and some of the methane gas started flowing out. The depletion experiment was stopped at 190 hours. The final pressure is $770 \mathrm{psi}$; the produced volume (brine and gas) was reported to be about $190 \mathrm{ml}$.

Figure 3.1.52 shows cross-sectional porosity and saturation distributions estimated from CT scans. Before hydrate formation $\left(2,500 \mathrm{psi}, 55^{\circ} \mathrm{F}\right)$, the average gas saturation was $30.9 \%(38.9 \mathrm{ml})$. After hydrate formation, another scan indicated an average hydrate saturation of $24.6 \%$ (30.8 $\mathrm{ml})$, and the rest 
was brine. Hydrate saturation varied axially, like the initial gas saturation. After dissociation, the average gas saturation was $54 \%(67.9 \mathrm{ml})$ and the rest was brine.

Figure 3.1.53 shows the electrical resistance between 4 electrodes placed along the length of the core. As the brine was injected before hydrate formation, electrical resistance decreased. At the onset of hydrate formation, the resistance between the E1 and E2 and E2-E3 electrodes decreased a small amount due to the increased brine saturation in the core. The core resistance stabilized for 5 days and increased when dissociation started. As brine was taken out during pressure depletion, electrical resistance increased.

Figure 3.1.54 shows the acoustic delay time in Test 12. CT images shown in Figure 3.1.55 indicate the presence of hydrate in the core. The darker colors indicate areas of lower densities (lower CT numbers), and the lighter colors indicate areas of higher densities (higher CT numbers). The red color indicates areas of gas saturation. The white color indicates areas of brine saturation. 
Figure 3.1.50. Test 12: Hydrate Formation with Constant Methane Mass

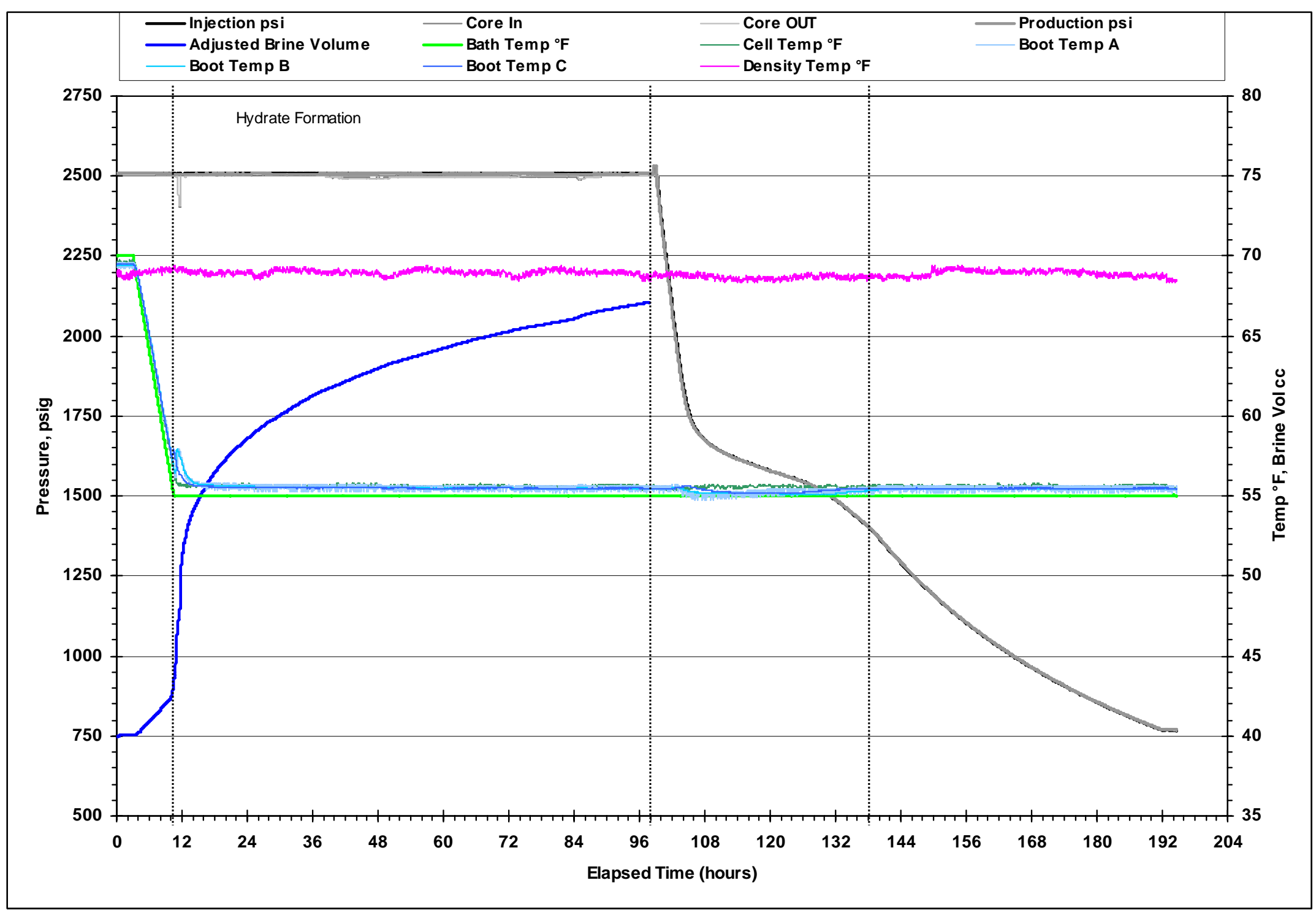


Figure 3.1.51. Test 12: Brine Injection, Depletion Volume and Production Density

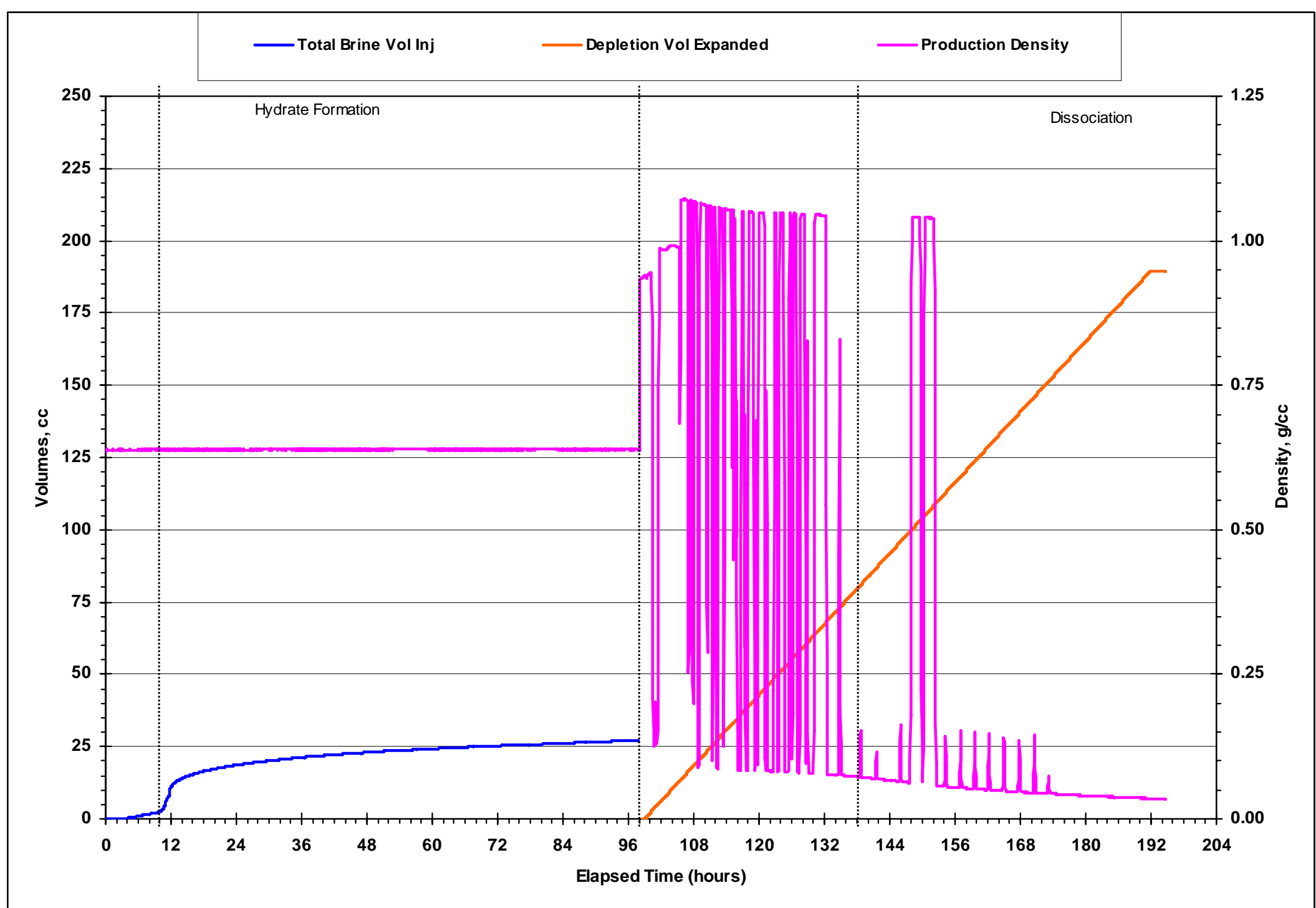


Figure 3.1.52. Test 12: Saturation and Porosity Derived from CT Scans

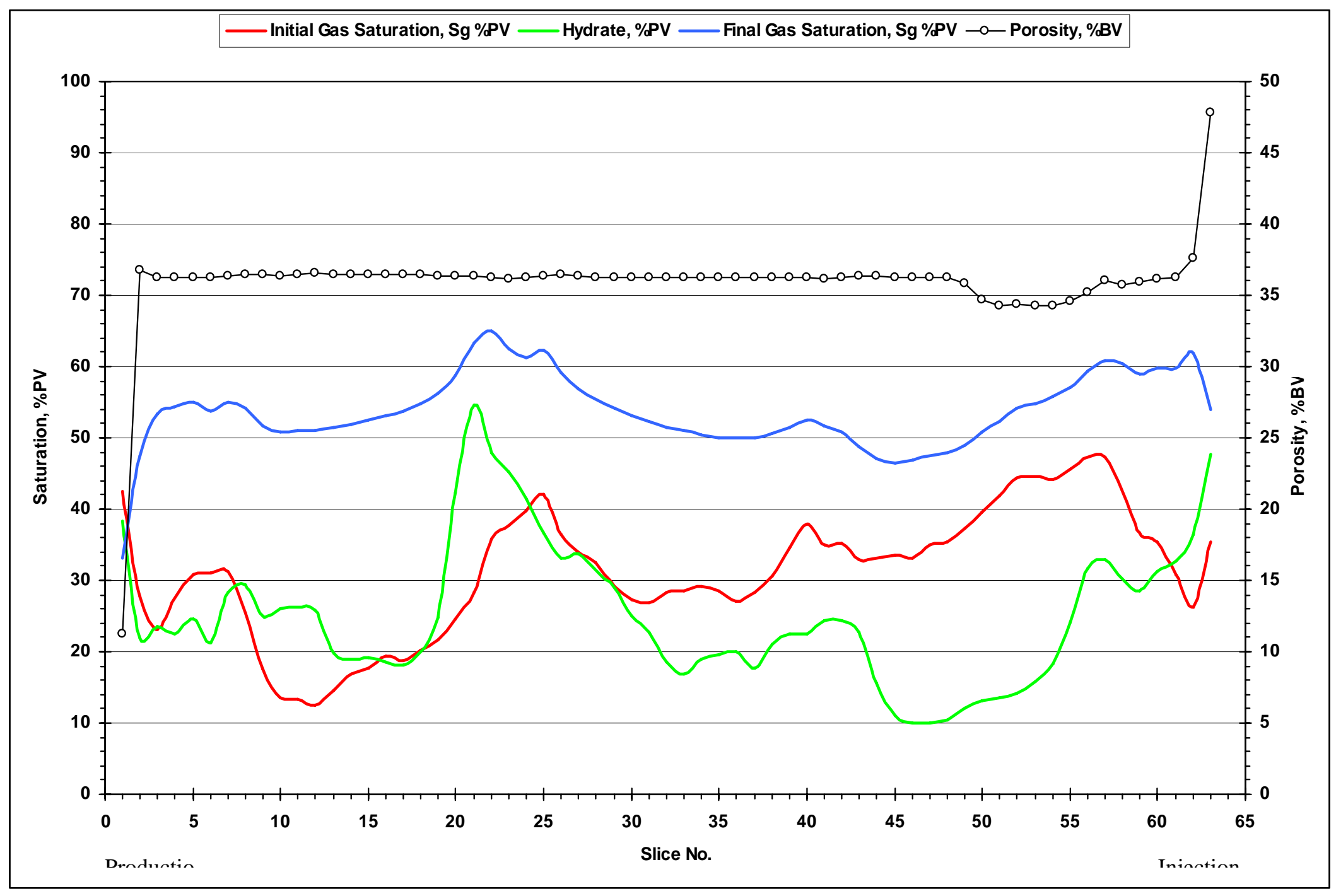


Figure 3.1.53. Test 12: Electrical Resistance, Brine Injection and Depletion Volume

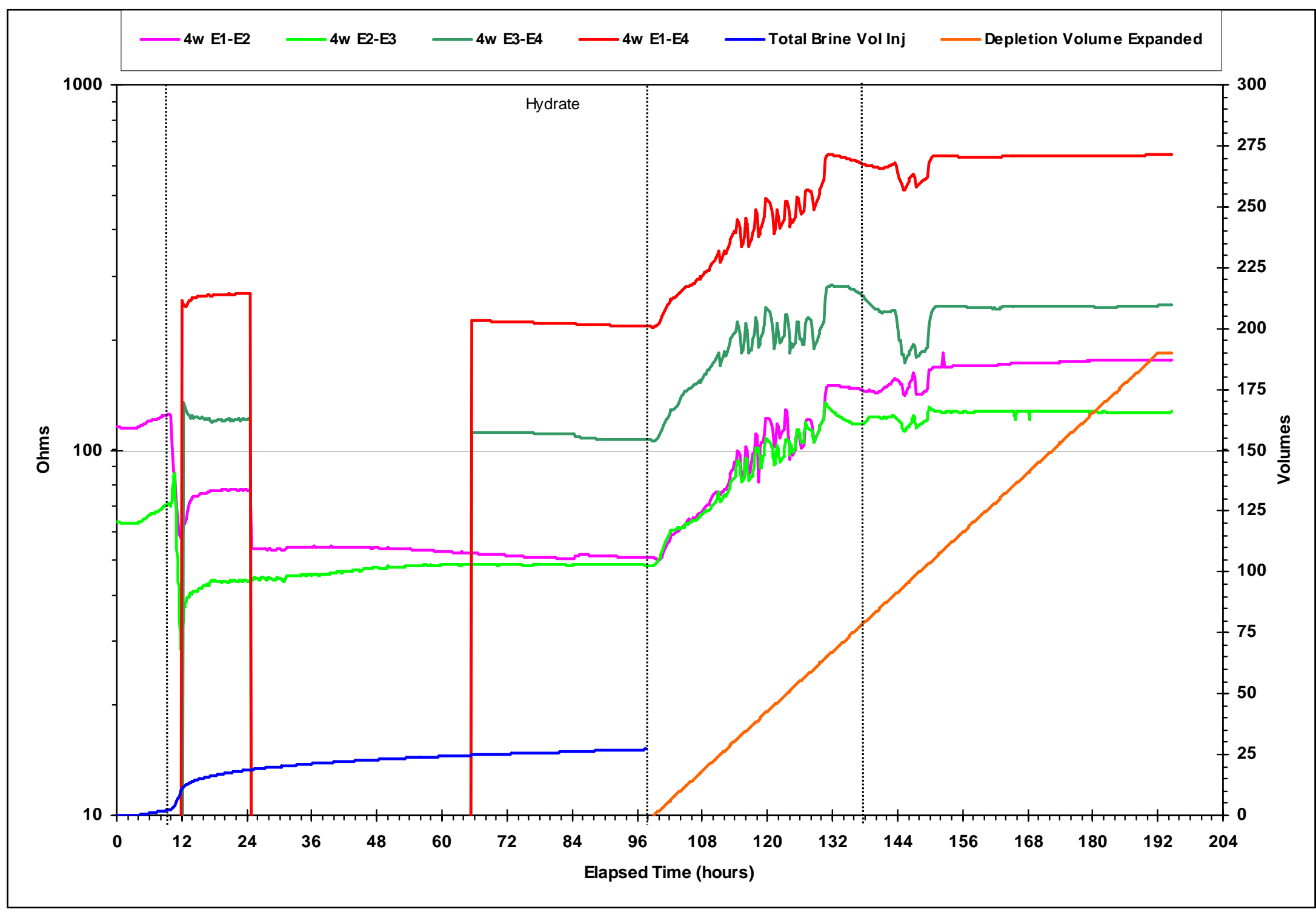


Figure 3.1.54. Test 12: Acoustic Delay Time, Brine Injection and Depletion Volume

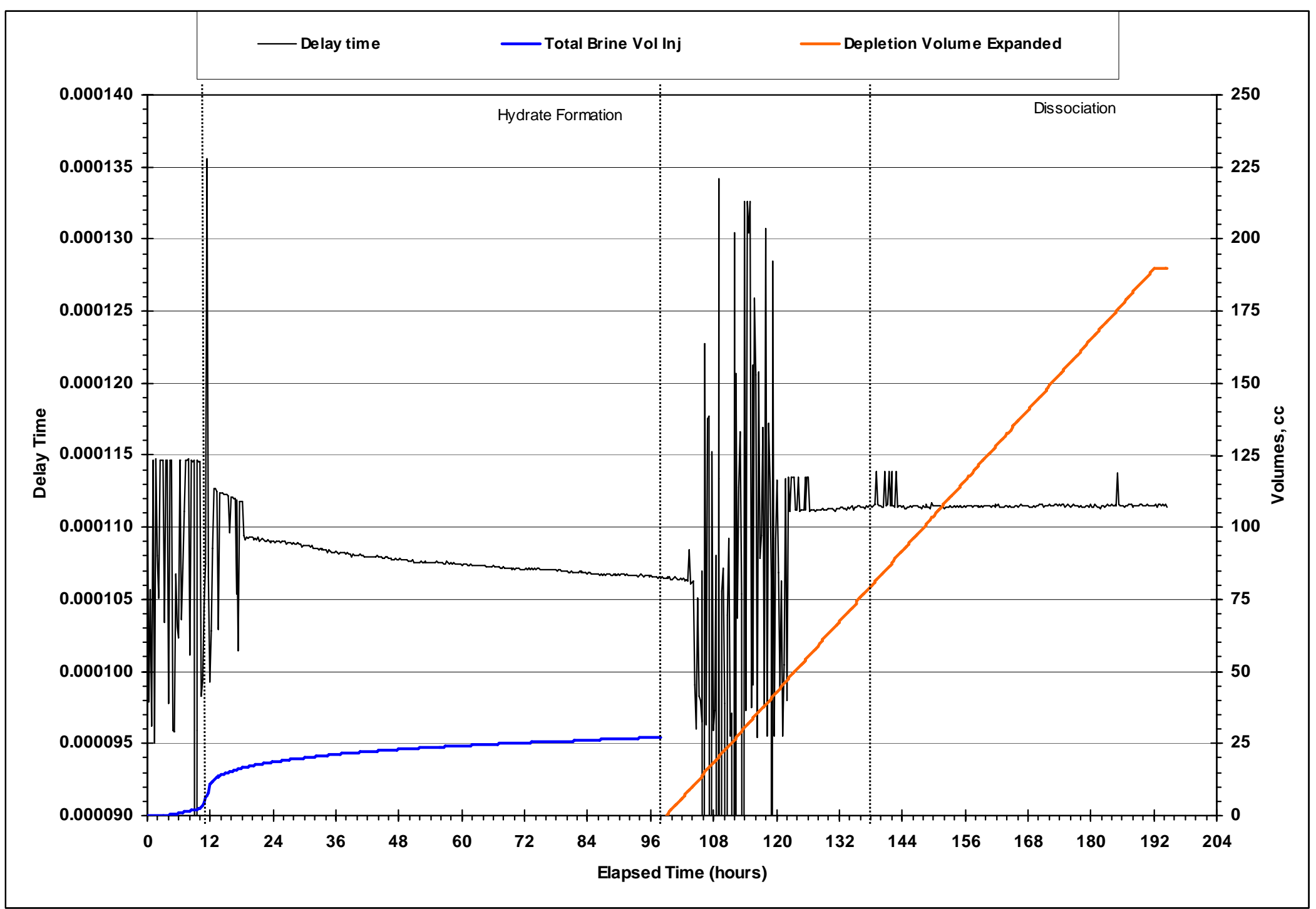


Figure 3.1.55. Test 12: CT Scan Results After Hydrate Formation
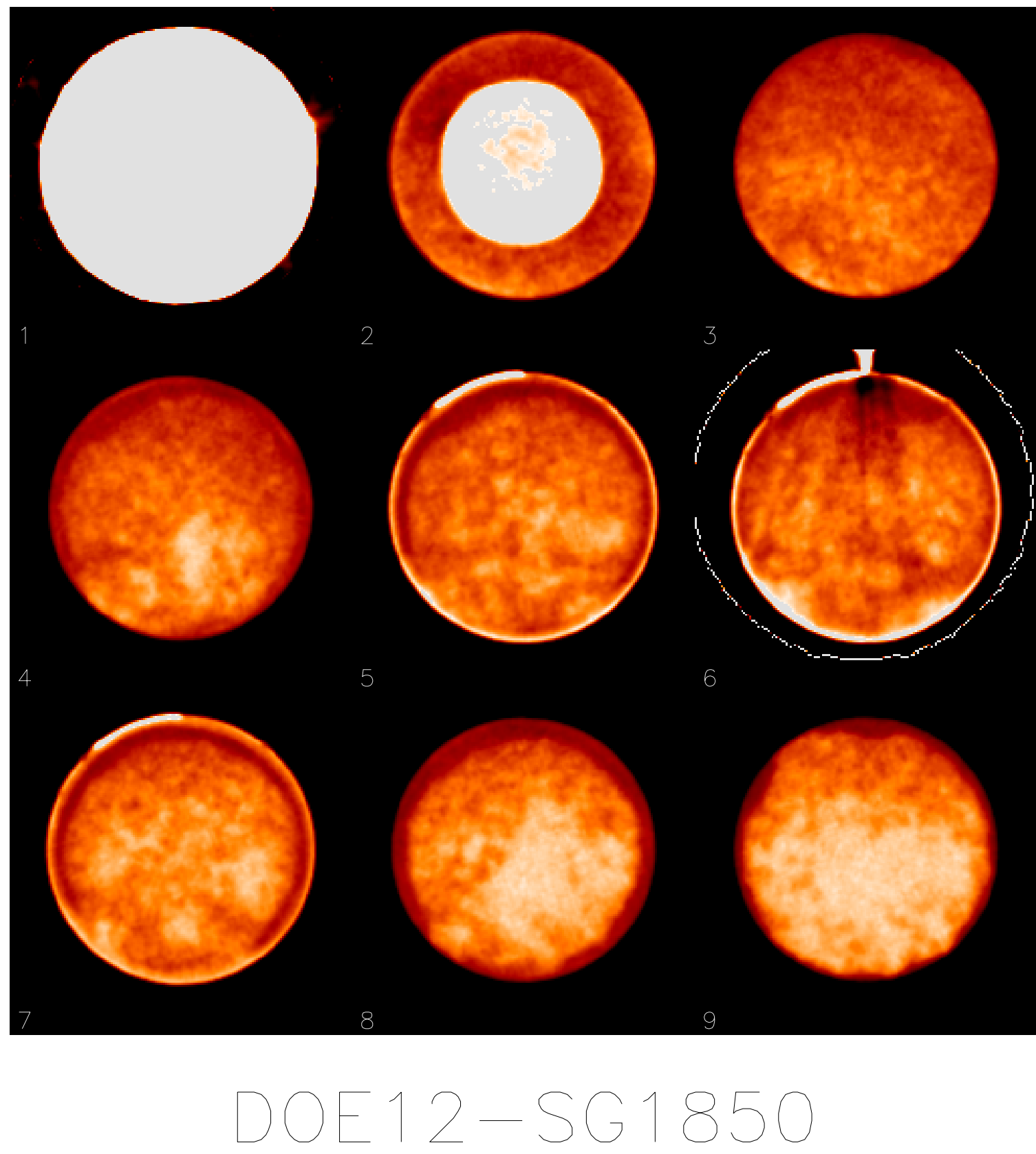

$5 \mathrm{~mm}$ slice thickness

700.0

1200.0

1700.0 


\subsubsection{Hydrate Formation in Simulated Gulf of Mexico Sediment (Test 13)}

Core Material:

Permeability (md):

Hydrate Formation Procedure:

Hydrate Dissociation Procedure:
Simulated GOM Sediment

Constant brine $\mathrm{P}$, decreasing $\mathrm{T}$

Pressure Depletion

Test 13 was the first test using a low permeability unconsolidated core. The inability to obtain representative GOM core samples from the Chevron Hydrate JIP described in Section 1.1.3 required a change in the final part of the program. Tests 2 through 12 had covered the range of higher porosities of formations that could be found in the GOM. However, experiments were needed to increase the accuracy of the simulator at the lower porosity range. A final series of experiments were designed using simulated unconsolidated core samples prepared based on the limited information from the Chevron Hydrate JIP available at the time.

A clean and dry 11.42 inch $(29.00 \mathrm{~cm})$ long by 1.5 inch diameter sand pack core material was mounted in the jacket and assembled in the core holder with the acoustical sensors, resistivity electrodes and RTD temperature sensors. The sand pack was held in place by two 1-inch long Berea sandstone cores on each end. This enabled good contact with the acoustic sensors. A net confining stress (NCS) of 500 psi was applied to the core. The core was saturated with $6 \% \mathrm{Nal}$ brine. The pore volume of the core was $123 \mathrm{cc}$ and the porosity was $46 \%$ of pore volume. CT scans were made of the core in the dry and brine saturated conditions. The core was de-saturated with methane gas to initial water saturation. The pore pressure was raised to 2,500 psi with methane while simultaneously raising the overburden pressure to 3,000 psi, maintaining a constant 500 psi NCS. Once at pressure, the system was leak tested for 3 days prior to the start of the test. All of these operations were carried out at room temperature.

A constant brine pressure test to form hydrate in the sand pack was begun. The cell was first cooled to $56^{\circ} \mathrm{F}$ at the rate of $2^{\circ} \mathrm{F} / \mathrm{hr}$. and allowed to equilibrate at that temperature for 2 hours and then further cooled to $56^{\circ} \mathrm{F}$ for 6 hours. Hydrate formation started at this cooling step. Additional cooling was completed to $42^{\circ} \mathrm{F}$ and then $36^{\circ} \mathrm{F}$. No additional indication of hydrate formation was seen at these temperatures. Figure 3.1.56 shows the temperatures, pump volume of gas injected and pressures for the entire test. Brine was injected into the core to maintain pressure.

Hydrate formation began at $46^{\circ} \mathrm{F}$. Like previous tests, the core temperature measured by the RTDs embedded in the jacket showed a sharp increase at the onset of hydrate formation. At the onset of hydrate formation as indicated by the RTDs, the pump began rapidly injecting gas (pump volume increasing) and the pore pressure began declining.

At the onset of hydrate formation, the resistance between the E1 and E2 and E2-E3 electrodes decreased a small amount due to the increased brine saturation in the core. The core resistance 
stabilized for 5 days and increased when dissociation started. Dissociation from the core was completed by pressure depletion.

This experiment was similar to Test 10 except that the core material was a simulated Gulf of Mexico sediment. The bath temperature was lowered to $39^{\circ} \mathrm{F}$ and then $33^{\circ} \mathrm{F}$ after hydrate formation at $55^{\circ} \mathrm{F}$. At 100 hours, hydrate formation was stopped and dissociation was started. The core temperature was at about $36^{\circ} \mathrm{F}$. The piston pump is withdrawn to decrease the pressure. The pressure and temperature are shown in Figure 3.1.56, and the piston withdrawal and the density of the effluent are shown in Figure 3.1.57. The withdrawal was $2 \mathrm{ml} / \mathrm{hr}$. Water flowed out at the start of the withdrawal. The pressure fell sharply. As the pressure reached the equilibrium brine-gas-hydrate pressure, hydrate dissociates and some of the methane gas started flowing out. In this case, dissociation occurred at much lower pressure ( $500 \mathrm{psi}$ ) because the temperature was lower $\left(36^{\circ} \mathrm{F}\right.$ vs. $\left.55^{\circ} \mathrm{F}\right)$. The depletion experiment was stopped at 247 hours. The final pressure was 350 psi; the produced volume (brine and gas) was about $300 \mathrm{ml}$.

Figure 3.1.58 shows cross-sectional porosity and saturation distributions estimated from CT scans. The porosity was not uniform because of the packing of loose sand. Before hydrate formation (2,500 psi, $\left.55^{\circ} \mathrm{F}\right)$, the average gas saturation was $18.3 \%(22.6 \mathrm{ml})$. The gas saturation varied along the porosity variation. After hydrate formation, another scan showed an average hydrate saturation of 5.1\% (6.3 ml) and the rest was brine. Hydrate saturation varied axially, like the initial gas saturation. After dissociation, the average gas saturation was $14.8 \%(18.2 \mathrm{ml})$ and the rest was brine. Figure 3.1 .59 shows the electrical resistance between 4 electrodes placed along the length of the core. As the brine was injected before hydrate formation, electrical resistance decreased. As brine was taken out during pressure depletion, electrical resistance increased.

Figure 3.1.60 shows a CT scan of the core after hydrate formation. The darker colors indicate areas of lower densities (lower CT numbers), and the lighter colors indicate areas of higher densities (higher CT numbers). The red color indicates areas of gas saturation. The white color indicates areas of brine saturation.

The experimental work was stopped by NETL in October 2005 while it considered its options on ending or completing this program. The work remaining was two repetitions of Test 13 and one repetition of Test 2. The decision to terminate the program was advised by a conference call by NETL in January 2006. However, when the decision was reversed in February 2006 to complete the program, the equipment had already been redeployed and the personnel reassigned to other projects. The cost to reassemble the equipment and run the last two experiments exceeded the remaining budget.

The data from all tests, including Test 13, were reviewed by Westport and the University of Houston. The data were also verified against the simulator and were determined to be valid. A comparison of the data with the simulator is presented in Section 3.2. It was determined that the program could be concluded with the data obtained. 
Figure 3.1.56. Test 13: Hydrate Formation with Constant Methane Mass

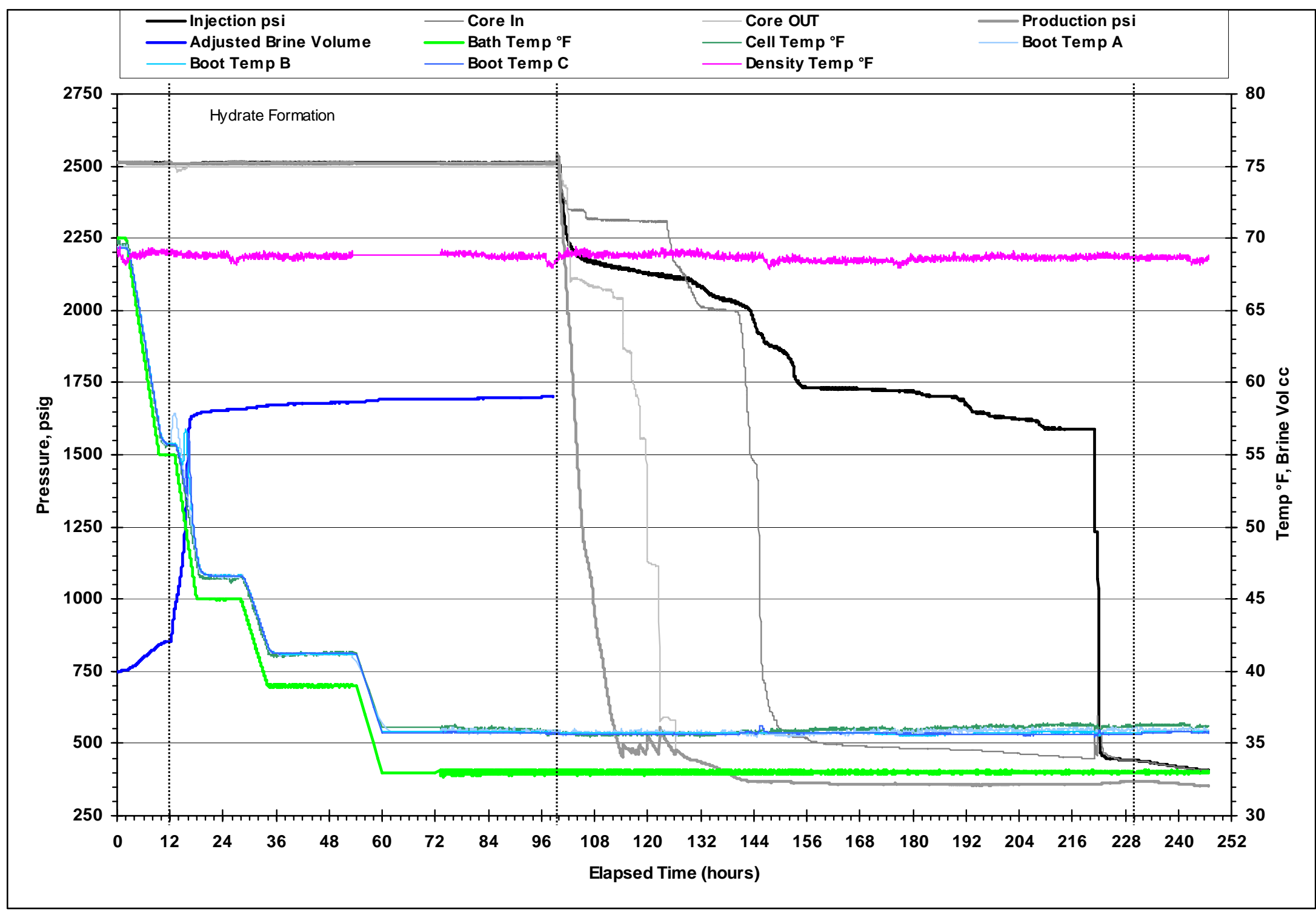


Figure 3.1.57. Test 13: Brine Injection, Depletion Volume and Production Density

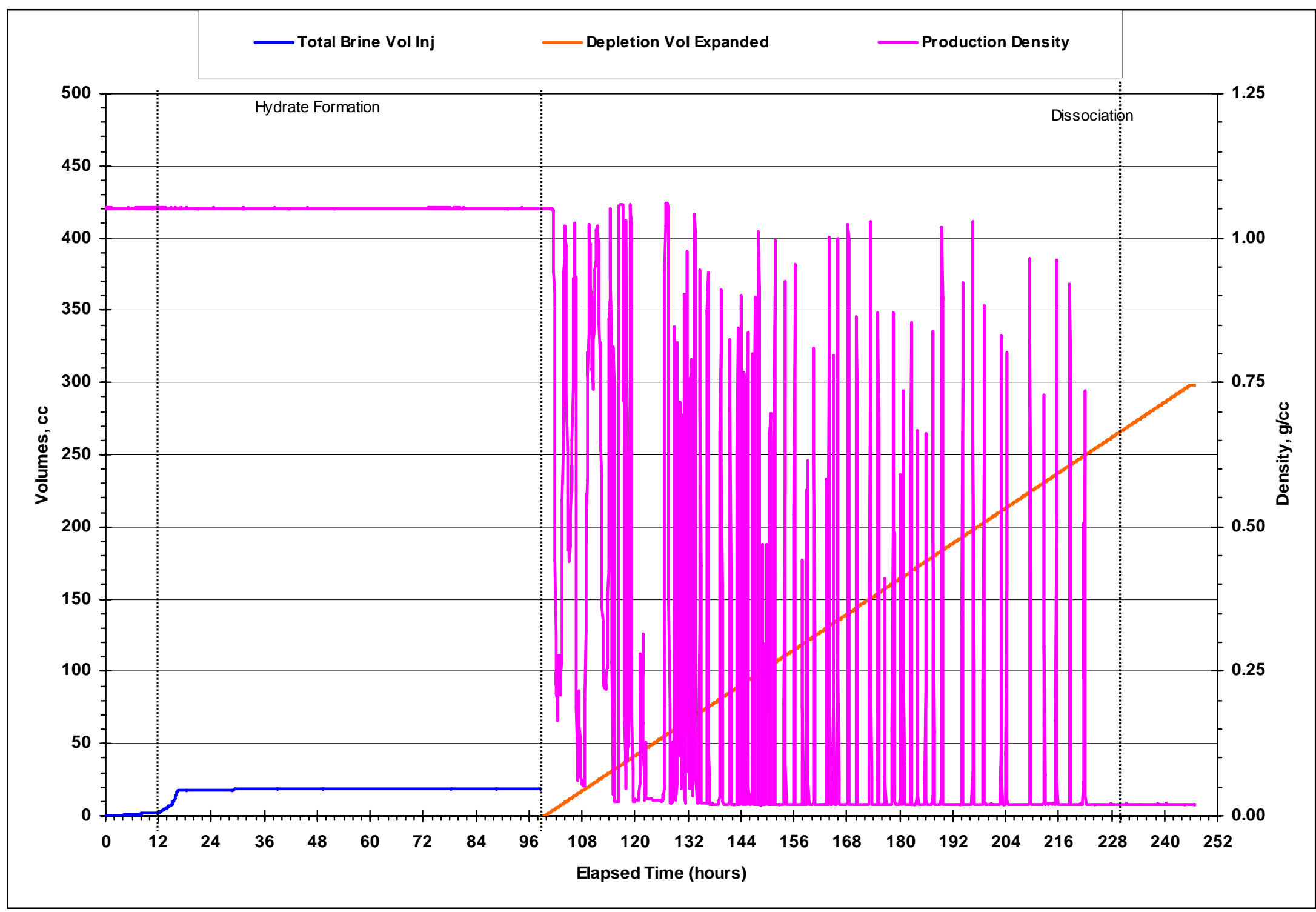


Figure 3.1.58. Test 13: Saturation and Porosity Derived from CT Scans

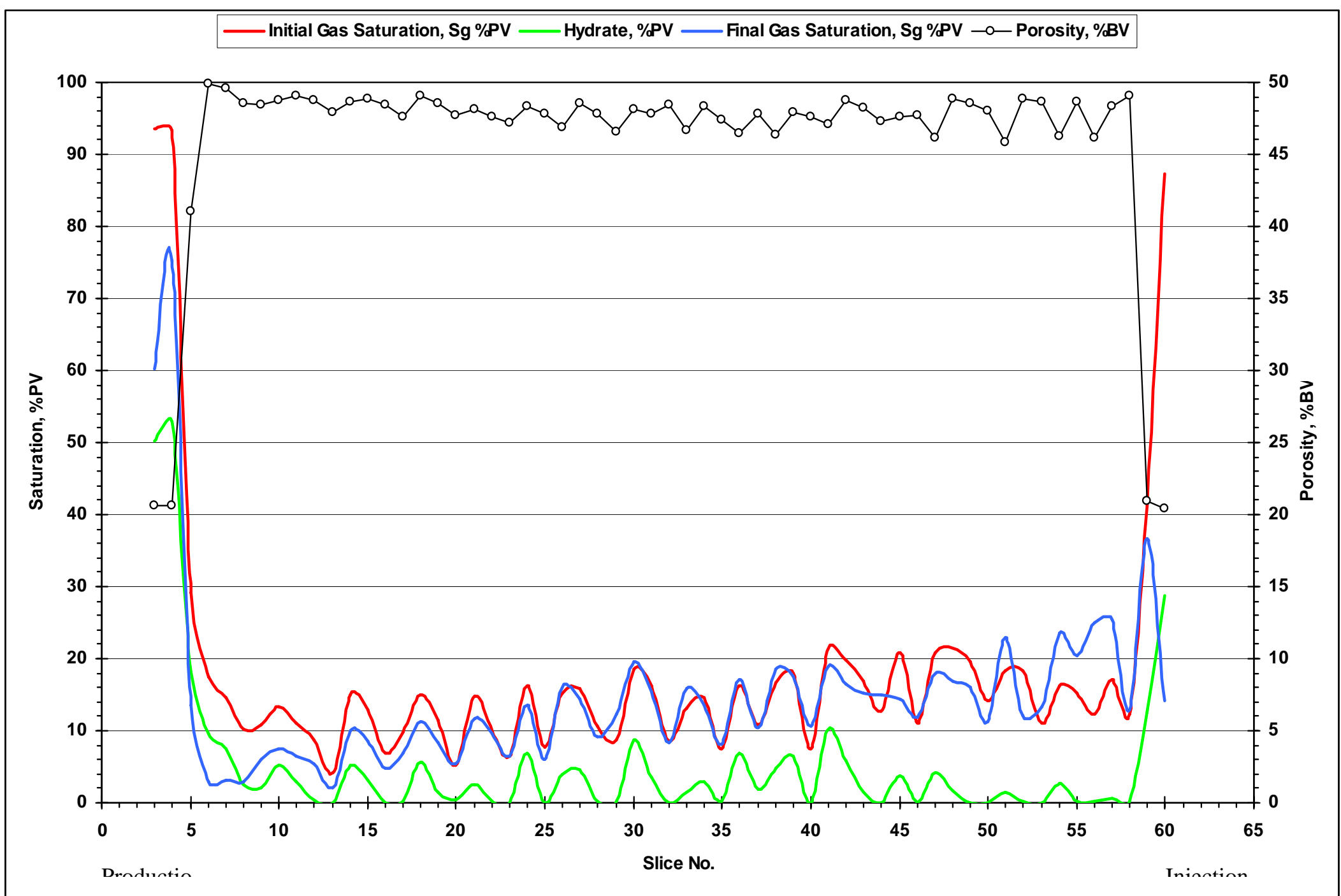


Figure 3.1.59. Test 13: Electrical Resistance, Brine Injection and Depletion Volume

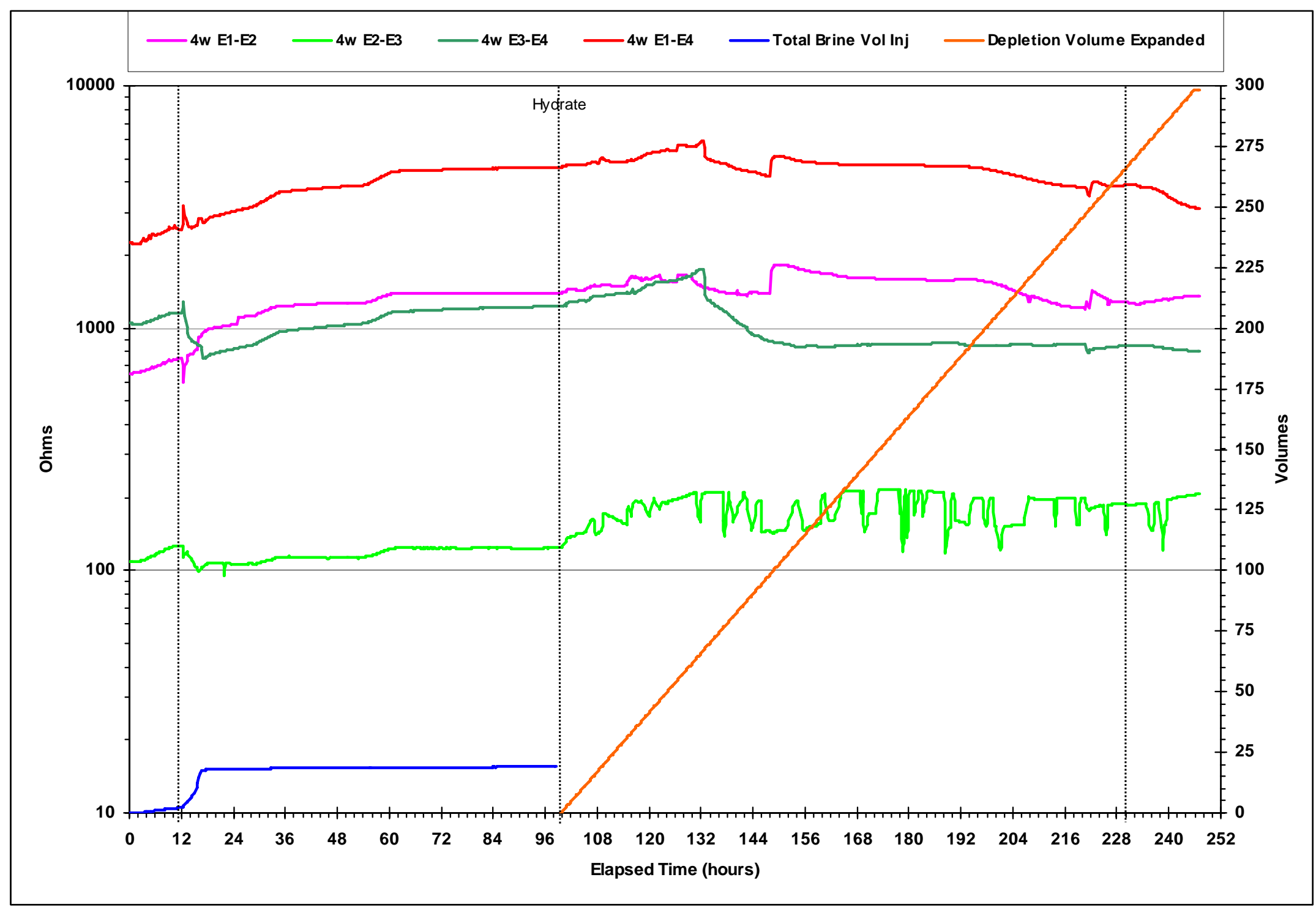


Figure 3.1.60. Test 13: CT Scan Results After Hydrate Formation
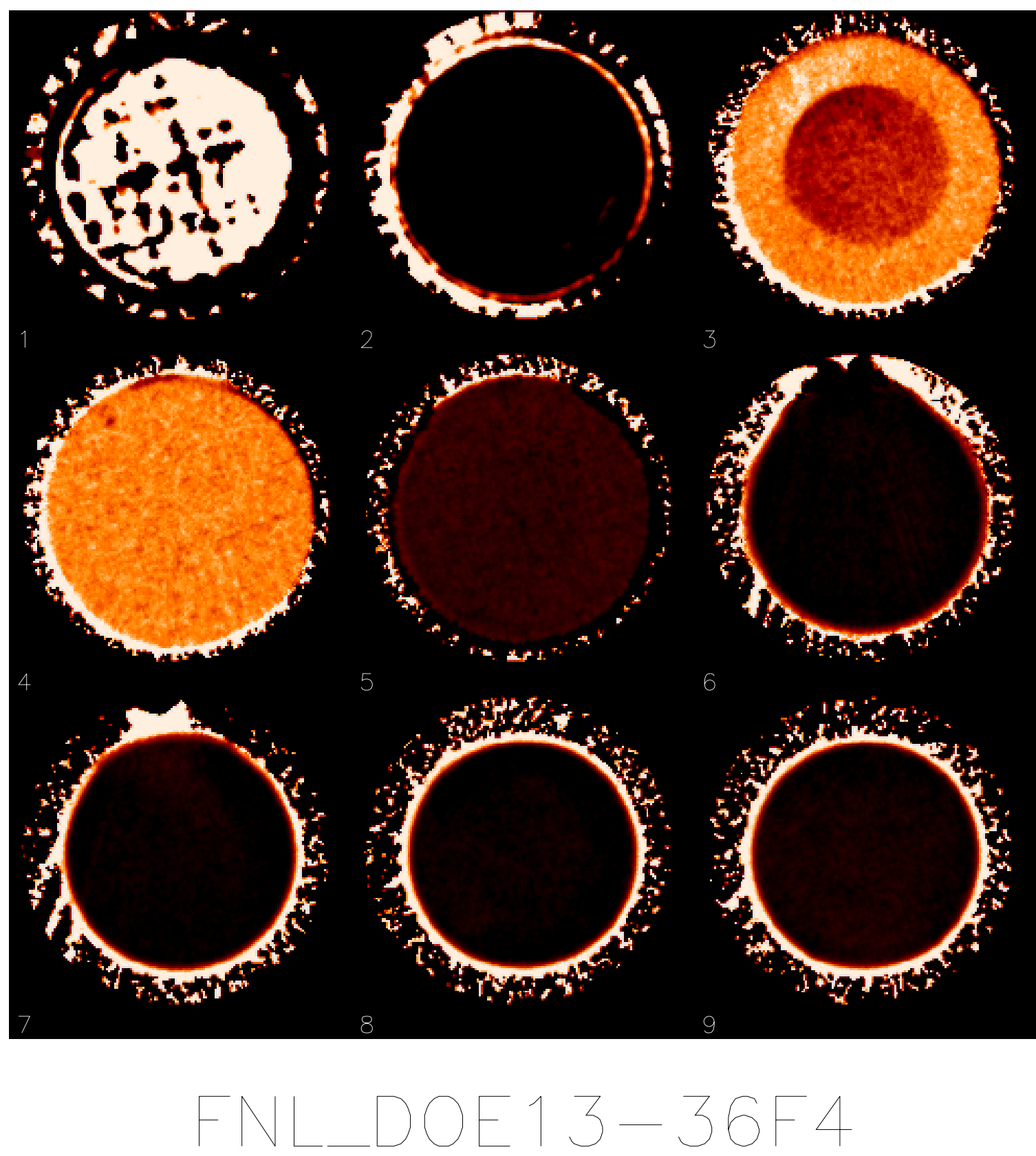

$5 \mathrm{~mm}$ slice thickness

50.0

100.0 


\subsubsection{Discussion}

The constant pressure approach was used for Tests 1-8, and the constant mass approach was used for all subsequent tests. Tests 1-12 were conducted in a porous ceramic core material. Test 13 was conducted with an unconsolidated core consisting of very fine sand to simulate Gulf of Mexico sediment.

Constant Methane Pressure. During Tests 1-8, the core was initially desaturated to a water saturation Sw of $\sim 50 \%$ with methane gas at low pressure. The system was then pressurized to 2,500 psia with methane gas and then cooled to $\sim 55^{\circ} \mathrm{F}$. A constant pressure of 2,500 psia was maintained during the cooling stage by regulating methane supply. Amount of methane gas pumped into the core was accurately measured with the pump (a small piston cylinder contained methane and backside pressure on piston was maintained at 2,500 psia with a pump that also measured water volume pumped to piston cylinder). Depressurization was performed by shutting off methane supply and withdrawing fluid at a fixed flow rate in some experiments and by increasing temperature in others.

Tests 1-4 and 6 were conducted using hydrate formation at constant methane pressure and dissociation by temperature increase. Tests 5,7 and 8 were to be conducted using hydrate formation at constant methane pressure and dissociation by constant volumetric rate depletion. Observations noted during tests 3-8 were that the resistance between the electrodes in the core sharply increased during hydrate association, indicating increased hydrate saturation.

Constant Methane Mass. Tests 10-13 were conducted using hydrate formation at constant methane mass and dissociation by constant volumetric rate depletion. The core was saturated with methane at a certain pressure and at ambient temperature. It was then injected with brine until the pressure reached $2,500 \mathrm{psi}$. At this state, the core was cooled by immersing the coreholder in a bath at $55^{\circ} \mathrm{F}$. As the fluids cooled, the volume decreased. The core was kept connected to a brine pump which kept the pressure constant at 2,500 psi during the cooling step by pumping brine. To initiate dissociation, the piston of a piston pump connected to one end of the core was withdrawn at a constant rate. Withdrawal of the piston lowered the pressure and fluids were drawn into the piston.

As the pressure dropped, the hydrates started to dissociate in each case. Methane gas and water were released. Brine was injected to maintain the pressure at 2,500 psi. A piston cell was used to remove fluids at a constant volumetric flow rate, which caused depressurization and hydrate dissociation. Observations noted during tests 10-13 were that the resistivity decreased during hydrate association, indicating increased brine saturation.

A comparison of the results of selected tests with the model is shown in Section 3.2, following. 


\subsection{Modeling Results}

Simulation results are presented in this section for experiments 10-13 as representative of the range of porosities expected in GOM formations. The key parameters for the simulations are listed below in Table 3.2.1. The core was discretized in R-Z cylindrical coordinates in $20 \times 20$ grid blocks. The layout of the grid blocks is shown in Figure 3.2.1. At maximum radius a boundary grid block was included (shown in brown color) which had zero porosity. This boundary was maintained at the temperature of the cooling bath for the core. This boundary was a no flow boundary.

Table 3.2.1. Key Parameters for the Simulations

\begin{tabular}{|l|l|}
\hline TEST 10 \\
\hline Rocks & $2.6 \mathrm{e} 3$ \\
\hline Density $(\mathrm{kg} / \mathrm{m} 3)$ & .36 \\
Porosity $(-)$ & $9.87 \mathrm{E}-13$ \\
Permeability (m2) & 3.1 \\
Formation heat conductivity (W/m C) & 1000. \\
Specific heat (J/kg C) & 6 \\
\hline Hydrate & 1 \\
\hline hydration number & .45 \\
mole fraction in composite hydrate & $2.1 \mathrm{e} 3$ \\
Coefficients in the thermal conductivity polynomial & $9.2 \mathrm{e} 2$ \\
Coefficients in the specific heat polynomial & $8.1 \mathrm{~d} 4$ \\
Coefficients in the density polynomial & $4.1 \mathrm{~d} 0$ \\
\hline Activation energy (J/mol) & $1.0 \mathrm{e}-0$ \\
intrinsic rate constant $\left(\mathrm{kg} \mathrm{m}^{-2} \mathrm{~Pa}^{-1} \mathrm{~s}^{-1}\right)$ &
\end{tabular}

\subsubsection{Comparison of Model Output to Test 10}

The well deliverability option was used to perform the depressurization. By specifying the bottomhole pressure in the well, it was possible to cause depressurization in the core and the methane can start to dissociate. A single well was created at one end of the core at its center grid block (AJO O in Figure 4.1). In the experiment, the water and gas together were pumped out of the core at a constant volumetric flow rate. However, in the current version of TOUGHFx/HYDRATE it was possible to create a sink with a constant mass flow rate but not a constant volumetric (combined aqueous and gas) flow rate. Therefore it was decided to apply the pressure boundary condition at the well bore.

Again, the simulator was designed to apply a constant pressure boundary condition, not a varying pressure boundary condition. Thus a set of consecutive runs (100 runs for each experiment) were conducted with step wise decrease in pressure which followed the pressure curve measured during the experiment. The depressurization pressure applied at the well as a function of time in Test 10 is shown in Figure 3.2.1. Manually entering the parameters of time and boundary conditions became a laborious process; therefore, a Matlab code was developed to read through the input file and change the start time 
and end time of the simulation along with the bottomhole pressure. After each small time step the simulator created a SAVE file which stored all the parameters of each grid block which was read by the following simulation. 100 directories for 100 pressure steps were created. Each directory had the input file with the appropriate time values and the boundary conditions. A batch file ran the simulator code and the $\mathrm{C}++$ data extraction code (TFXRead) on each of the input files and copied the SAVE file into the next directory. Each set of simulations took approximately 45 minutes to run on a PC under Windows XP.

It was noticed from the experimental results that the initial amount of hydrate (before the start of depressurization) could be estimated from the brine injected into the core during hydrate formation. For example, $\sim 25 \mathrm{ml}$ of brine was injected in Test 10 (Figure 3.1.30). From the hydrate reaction stoichiometry, one could estimate that $\sim 25 \mathrm{ml}$ of brine reacted with $\sim 31.3 \mathrm{ml}$ of methane at 2,500 psi to form $\sim 31.3 \mathrm{ml}$ of hydrate at $2,500 \mathrm{psi}, \sim 55^{\circ} \mathrm{F}$. The CT scans estimated the hydrate volume in the core to be $25.6 \mathrm{ml}$ in Test 10. The CT hydrate saturations were not totally reliable because the salt concentration of the brine changed during hydrate formation. Test 10 was simulated assuming that the hydrate volume was $31.3 \mathrm{ml}$.

In Test 10, Figure 3.1 .30 indicated that about $275 \mathrm{ml}$ of brine and gas was produced at the final pressure of about $625 \mathrm{psi}$. It can be shown that $31.3 \mathrm{ml}$ of hydrate cannot produce $275 \mathrm{ml}$ of gas and brine at 625 psi; the production amount will be much less (about a half that amount). It was concluded that there was additional gas volume in the core, core holder fittings, and the outlet piston; part of this volume was connected to the piston and part may have been initially disconnected (but connected after hydrate dissociation). The additional connected gas volume was estimated to be $35.3 \mathrm{ml}$ and the disconnected volume was $19.9 \mathrm{ml}$. Adding the expansion of these two gas volumes to that produced from the core simulation provided the estimate of the total gas produced.

The experiments were conducted with sodium iodide brine. The hydrate phase behavior with sodium iodide is not available in published literature. To simulate, it was assumed that the inhibitor concentration in the water was $1 \%$ so that the pressure of hydrate dissociation matched that of the experiments.

Figures 3.2.2 - 3.2.8 show the simulation results for Test 10. Figure 3.2.2 shows the variation of the average pressure in the core with time. Shown as an error bar is the standard deviation in pressure among different grid blocks. The average pressure tracked the well pressure. The pressure fell sharply before hydrate dissociation indicating expansion of gas and expulsion of brine. The pressure fell slowly during hydrate dissociation indicating release of gas to fill up the volume created by the withdrawal of the piston. The pressure fell at a faster rate after the hydrate dissociation due to the expansion of gas. The error bars were small after hydrate dissociation, indicating small pressure drops within the core.

Figure 3.2.3 shows the variation of temperature within the core. The average temperature is shown as the 
middle line. The variation in the temperature is shown as an error bar. The temperature was more or less constant before 0.4 days, fell during the hydrate depressurization, and eventually equilibrated to boundary temperature. This behavior matched the experimental observation in Test 10 (Figure 3.1.29). During the hydrate depressurization, the latent heat was extracted from the vicinity of the hydrate which lowered the temperature.

Figure 3.2.4 shows the hydrate saturation within the core. The average saturation is plotted as the middle line. The variation in hydrate saturation is shown as an error bar. The average saturation started to decrease after 0.15 days. Most of the hydrate dissociated by 0.9 days. This behavior matched the experimental observation in Test 10 (Figure 3.1.31). Again the variation in hydrate saturation was small. Figure 3.2.5 shows the water saturation within the core. The average saturation is plotted as the middle line. The variation in water saturation is shown as an error bar. The average saturation started to decrease from the beginning, but decreased faster after 0.15 days. Most of the hydrate dissociated by 0.9 days; and the saturation did not change much afterwards. The variation in water saturation was significant and increased with time.

Figure 3.2.6 shows the gas saturation within the core. The average saturation is plotted as the middle line. The variation in gas saturation is shown as an error bar. The average saturation started to increase after 0.15 days at the onset of hydrate dissociation. Most of the hydrate dissociated by 0.9 days; and the saturation did not change much afterwards. The variation in gas saturation was significant and increased with time.

Figure 3.2.7 shows the total volume of hydrate, aqueous, gas and ice in the core. The hydrate has completely dissociated after 0.85 days. No ice formed in this simulation. Figure 3.2.8 shows the cumulative amount of methane production with time at the pressure in the piston after accounting for the expansion of connected and disconnected gases present. This data can be compared with the experimental data shown in Figure 3.1.30. The gas production was approximately constant after accounting for the water production. It can be concluded that the simulation was able to predict the experiments qualitatively. 
Figure 3.2.1. Test 10: Pressure Stepping;

The Blue Line is the Data from the Experiments and the Red Points

are the Points at which the Simulations were Performed

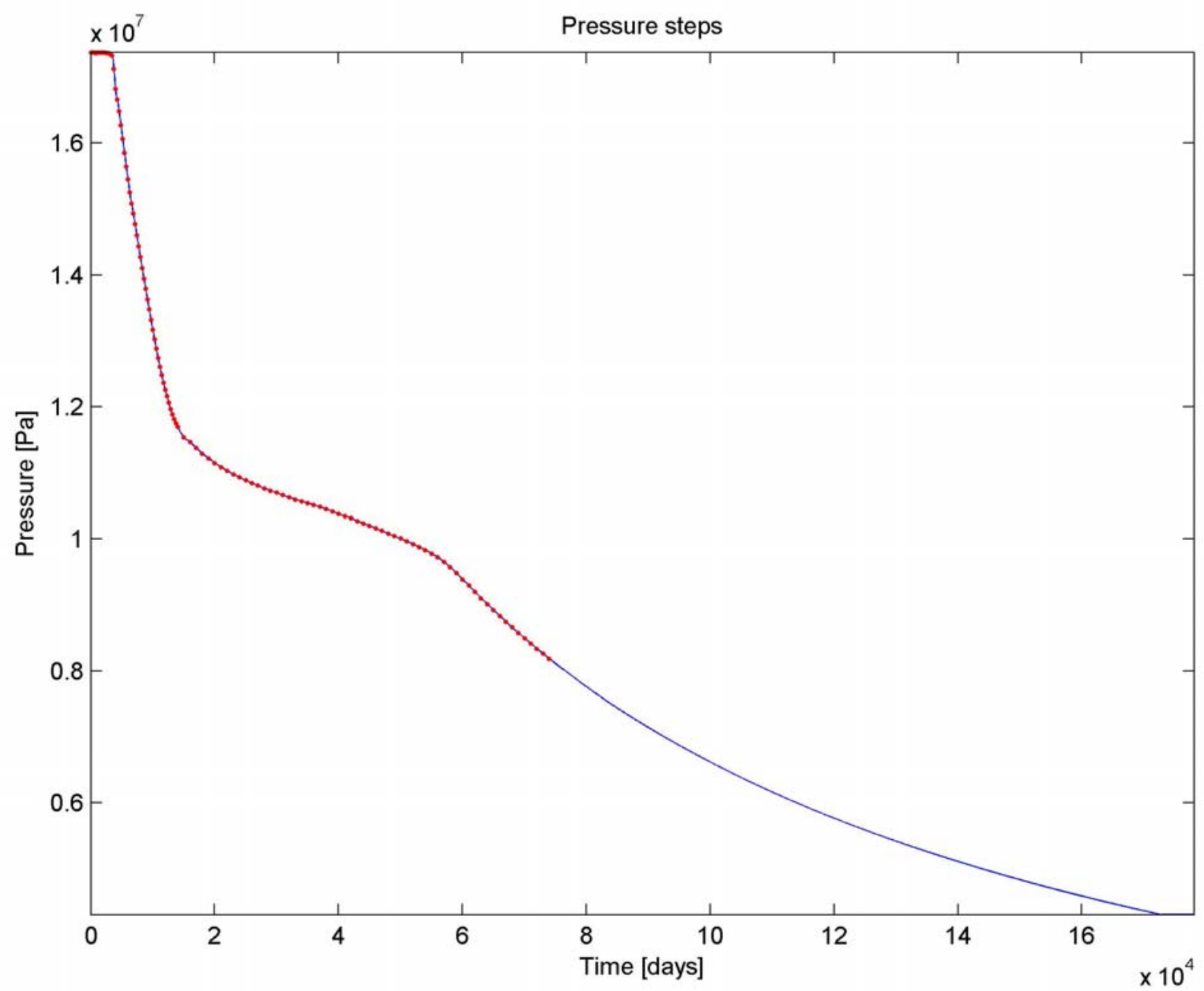


Figure 3.2.2. Test 10: Average Pressure throughout the Core with Time; The Error Bar is the Variation in all the Grid Blocks

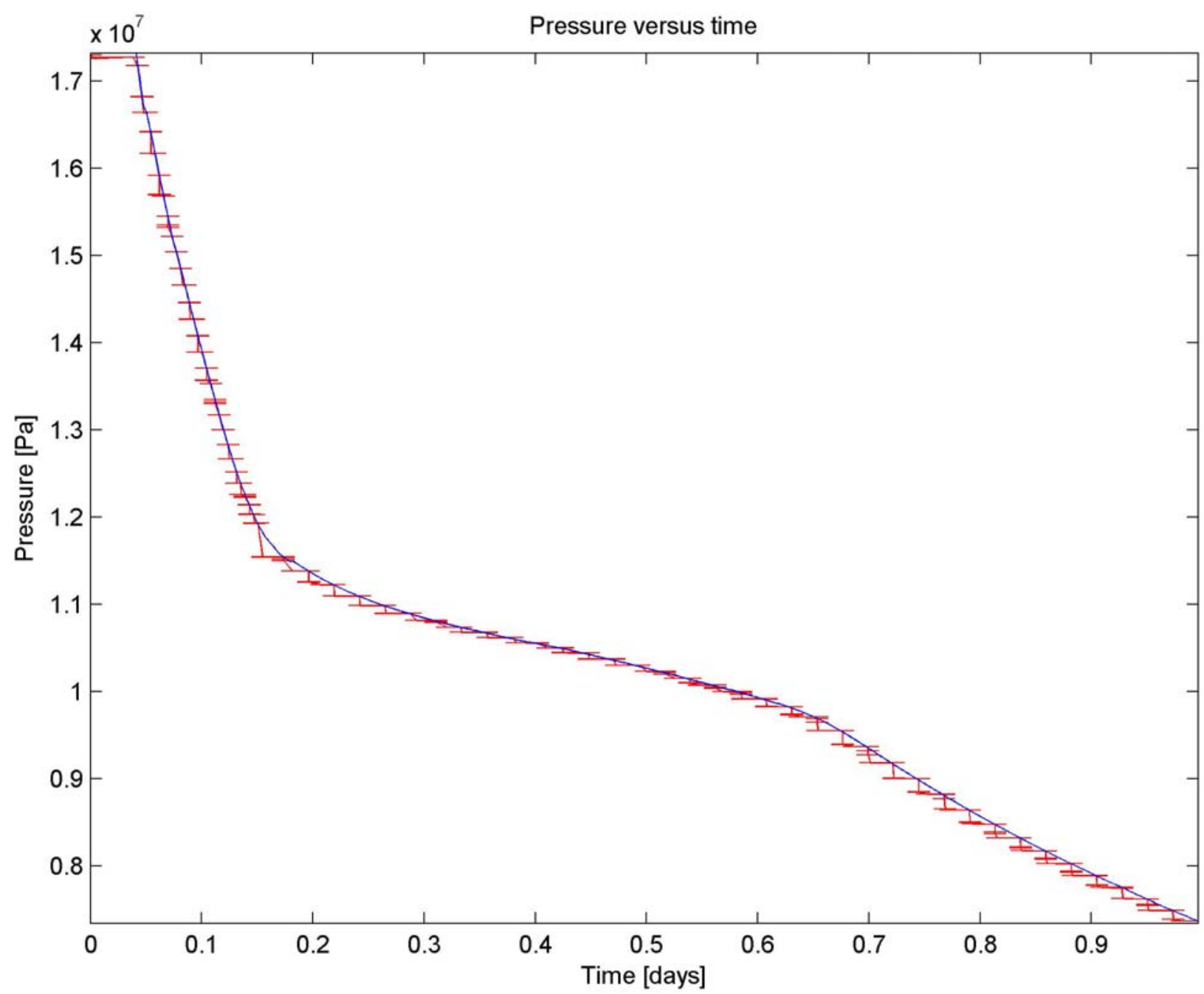


Figure 3.2.3. Test 10: Average Temperature throughout the Core with Time; The Error Bar is the in all the Grid Blocks

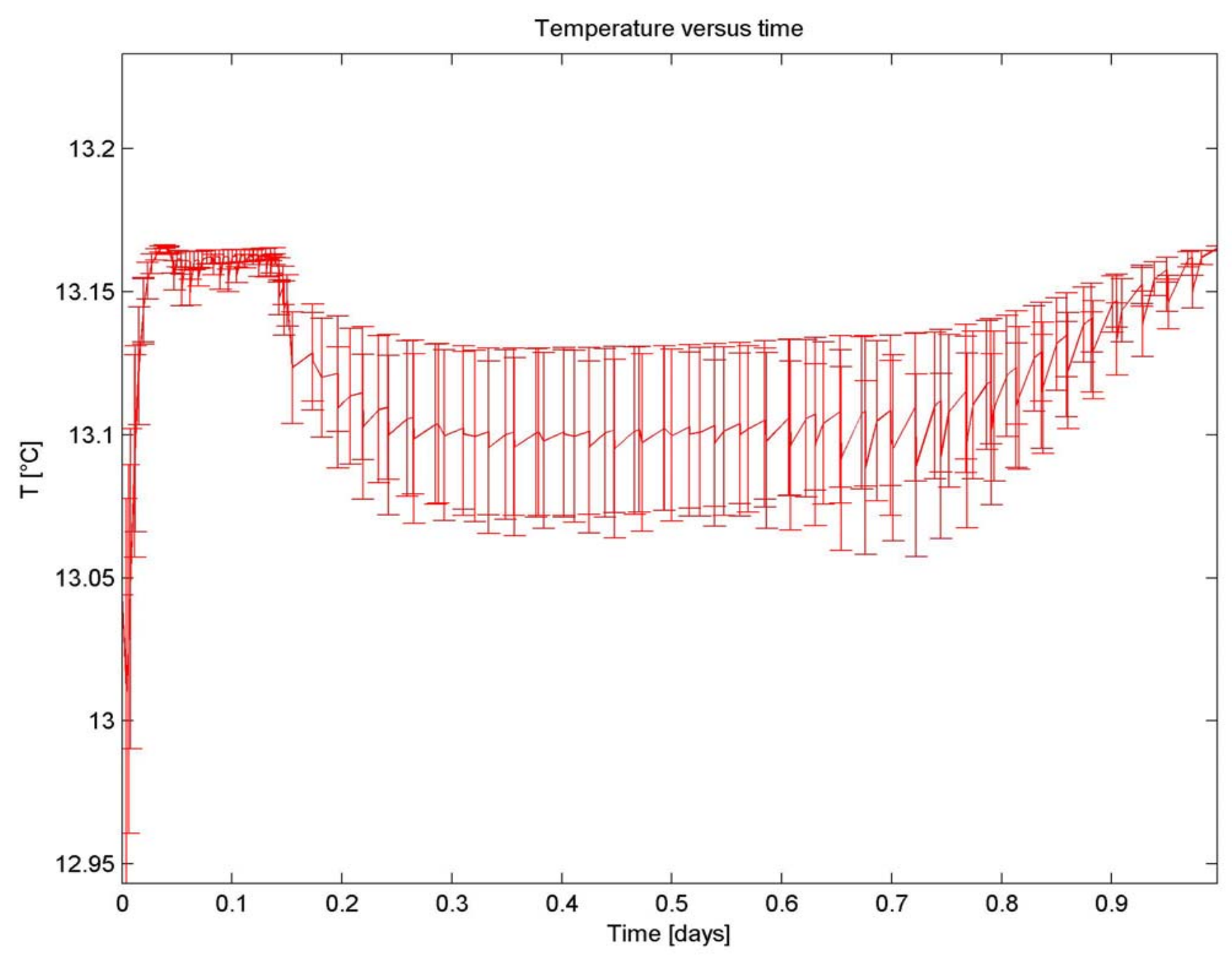


Figure 3.2.4. Test 10: Average Hydrate Saturation throughout the Core with Time; The Error Bar is the Variation in all the Grid Blocks

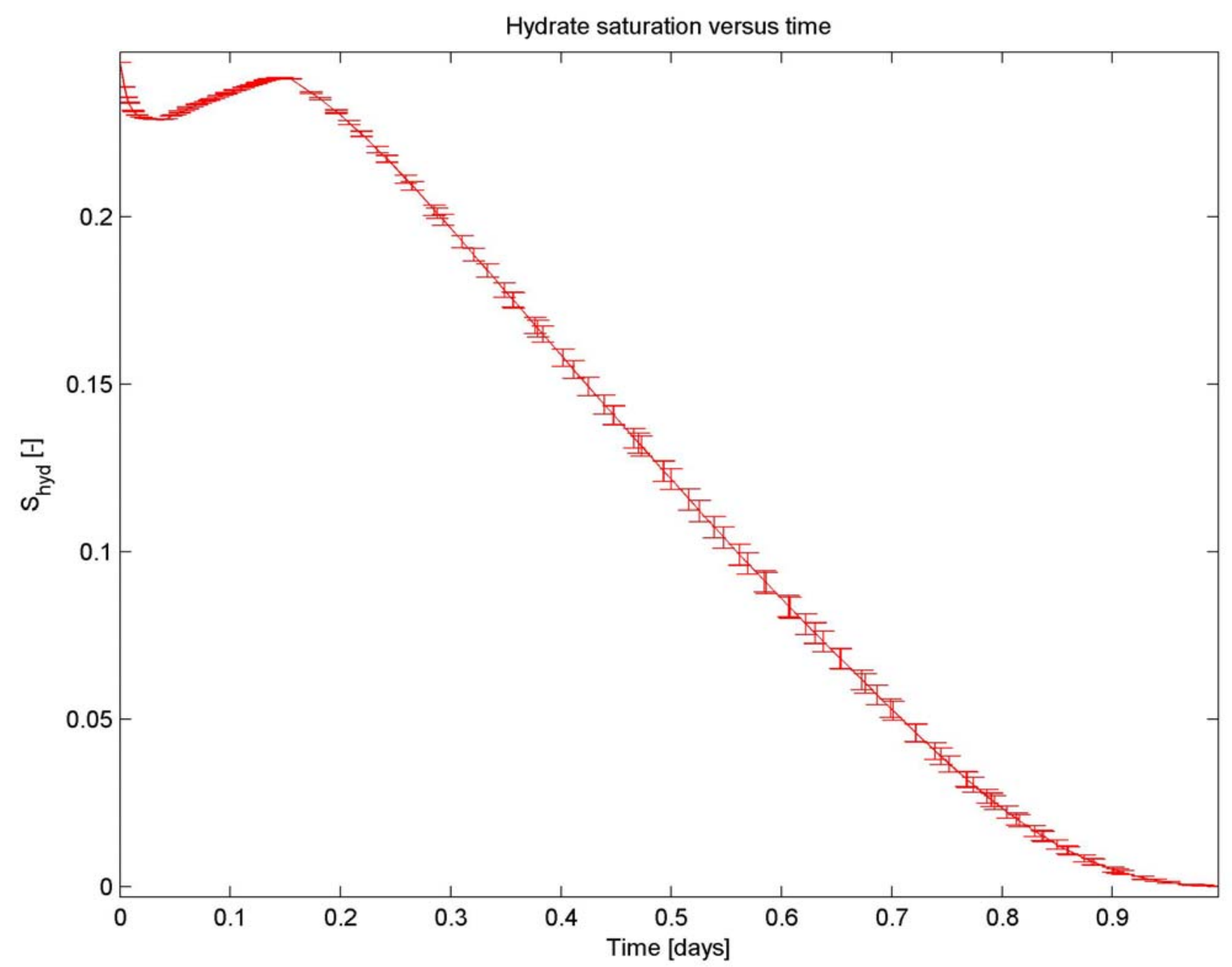


Figure 3.2.5. Test 10: Average Aqueous Saturation throughout the Core with Time; The Error Bar is the Variation in all the Grid Blocks

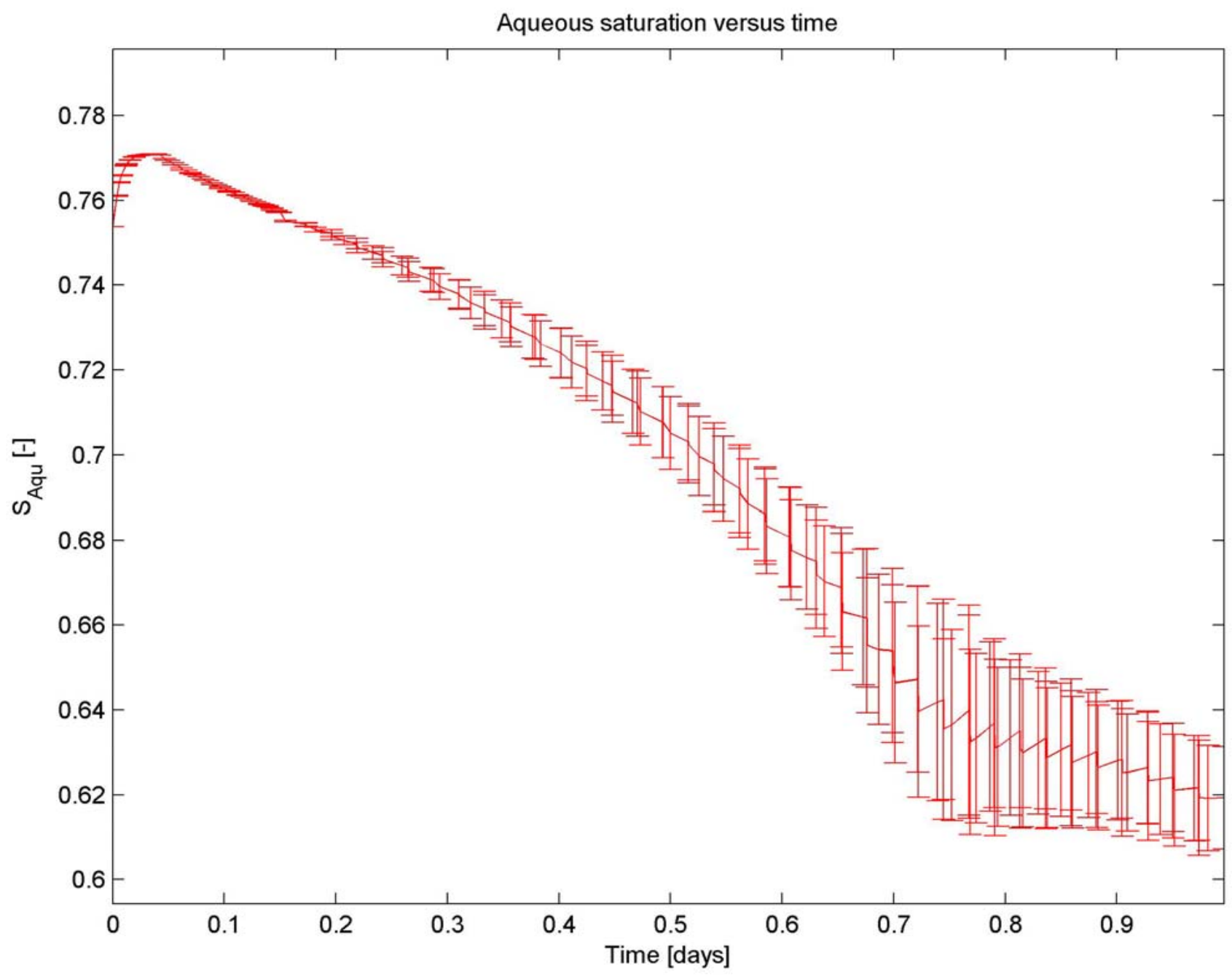


Figure 3.2.6. Test 10: Average Gas Saturation throughout the Core with Time; The Error Bar is the Variation in all the Grid Blocks

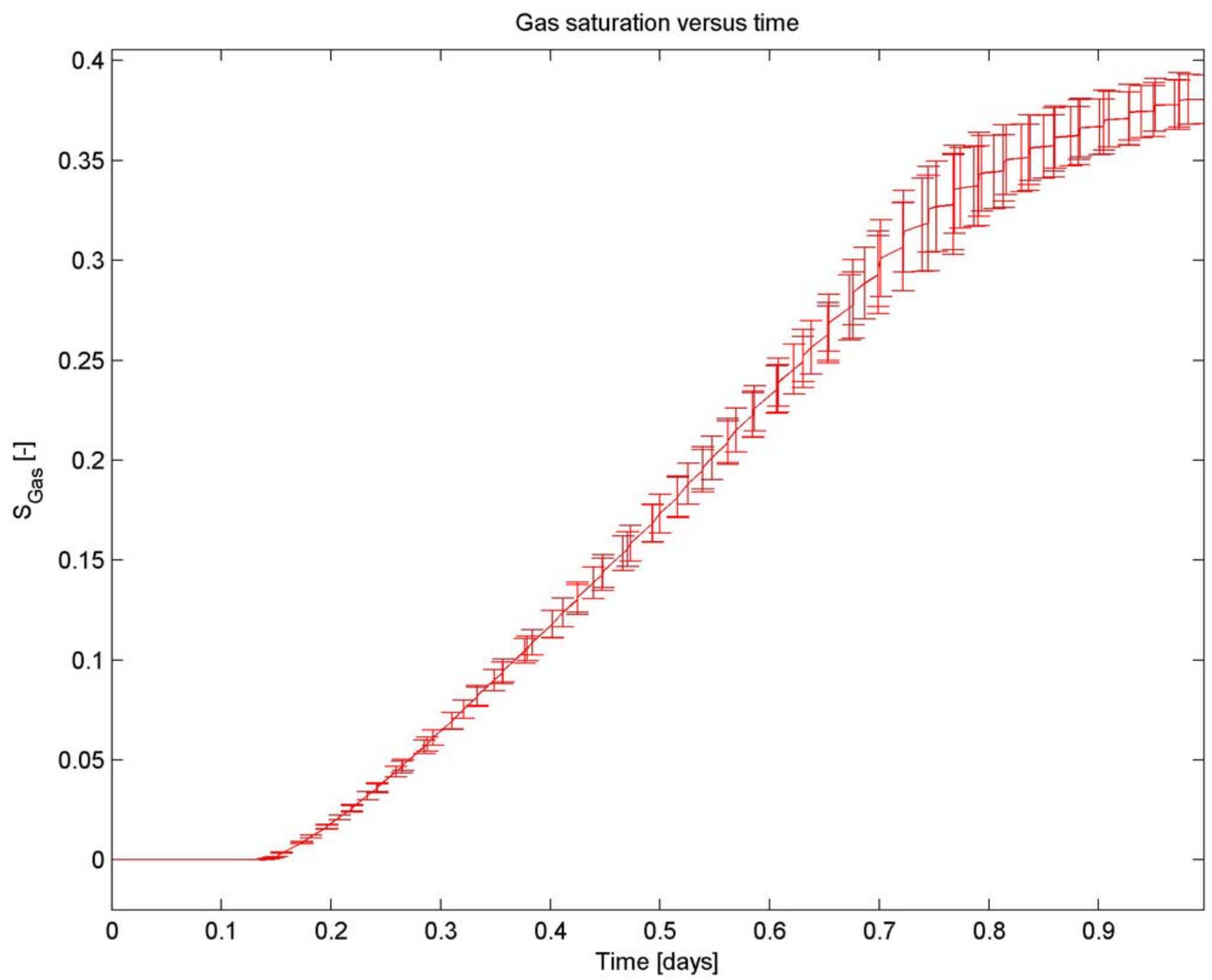


Figure 3.2.7. Test 10: Total Volume of Gas, Hydrate, Aqueous and Ice in the Core with Time; The Error Bar is the Variation in all the Grid Blocks

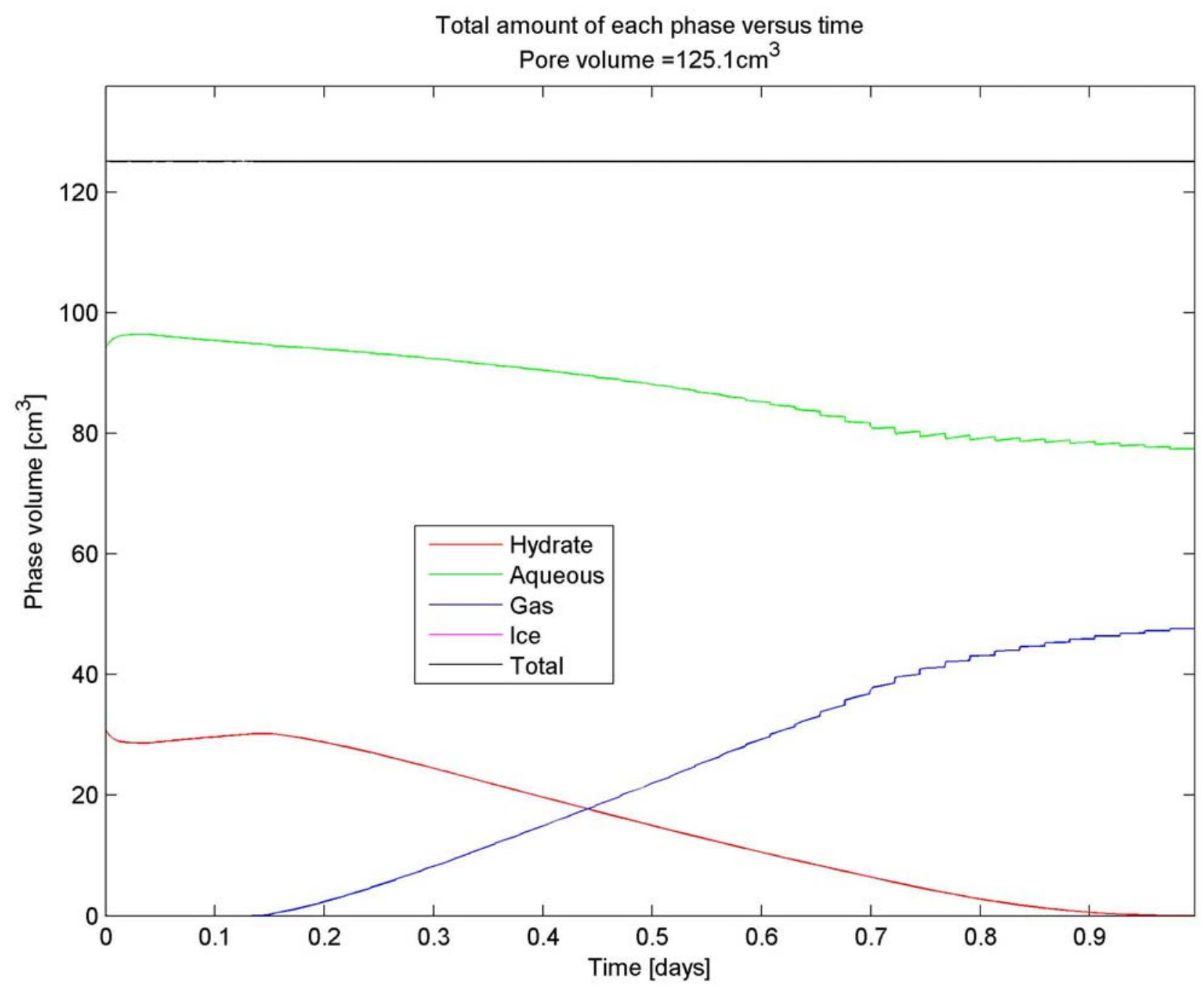


Figure 3.2.8. Test 10: Cumulative Volume of Methane Released over a Period of Time

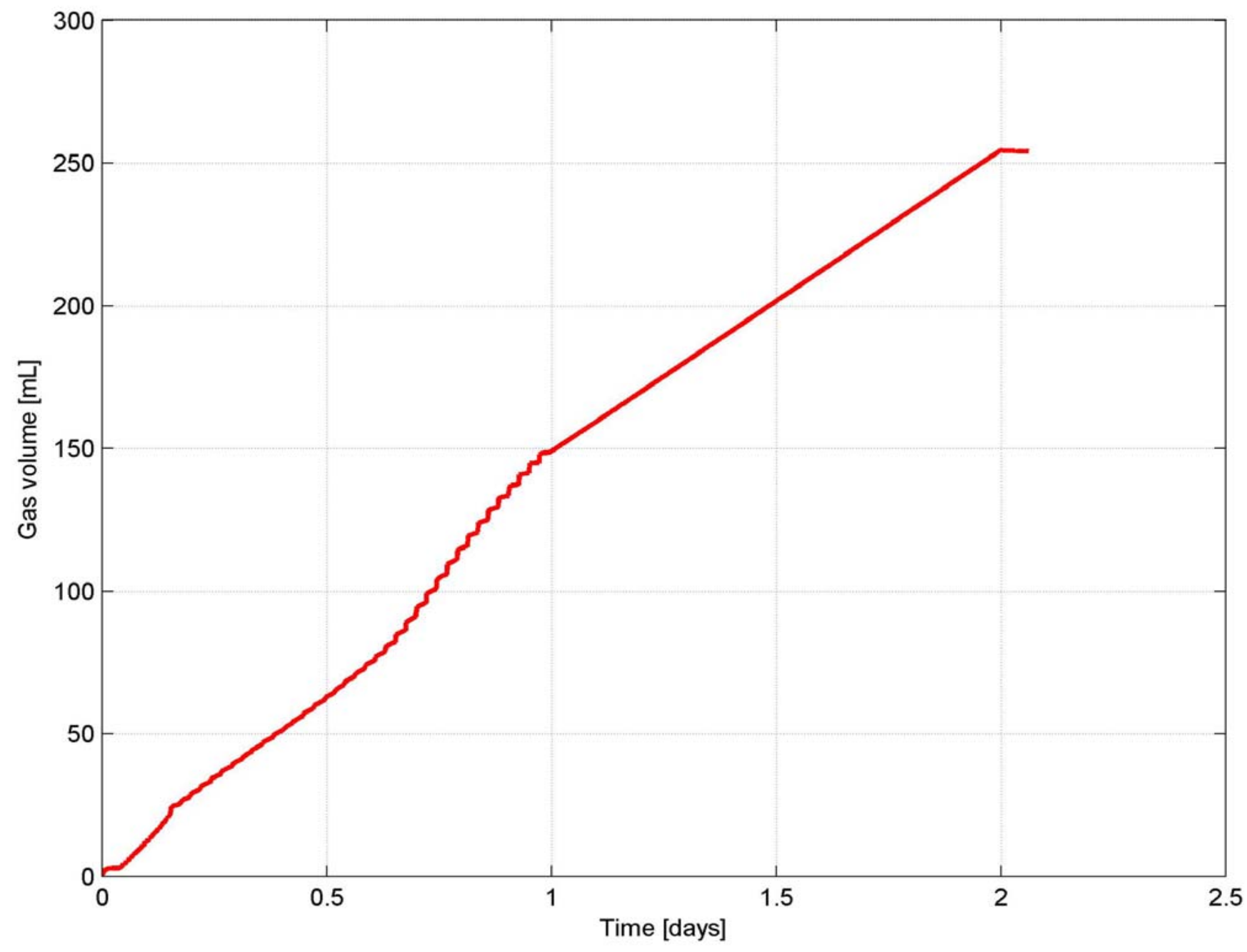




\subsubsection{Comparison of Model Output to Test 11}

Figures 3.2.9 - 3.2.15 show the simulation results for Test 11. In this test the depressurization was conducted at a much slower rate than Test 10. Also during the formation, there was some malfunction and resulting pressure drop for a short time which had led to more efficient hydrate formation. The initial connected gas volume was $24.5 \mathrm{ml}$ and disconnected volume was $0 \mathrm{ml}$. Figure 3.2.9 shows the variation of the average pressure in the core with time. What is shown as an error bar is the standard deviation in pressure among different grid blocks. The average pressure tracked the well pressure. The pressure fell sharply before hydrate dissociation indicating expansion of gas and expulsion of brine. The pressure fell slowly during hydrate dissociation indicating release of gas to fill up the volume created by the withdrawal of the piston. The pressure fell at a faster rate after the hydrate dissociation due the expansion of gas, again. The error bars are small after hydrate dissociation, indicating small pressure drops within the core.

Figure 3.2.10 shows the variation of temperature within the core. The average temperature is shown as the middle line. The variation in the temperature is shown as an error bar. The temperature was more or less constant before 0.4 days, fell during the hydrate depressurization, and eventually equilibrated to boundary temperature. This behavior matched the experimental observation in Test 11 (Figure 3.1.35). During the hydrate depressurization the latent heat was extracted from the vicinity of the hydrate which lowered the temperature.

Figure 3.2.11 shows the hydrate saturation within the core. The average saturation is plotted as the middle line. The variation in hydrate saturation is shown as an error bar. The average saturation started to decrease after 0.4 days. Most of the hydrate dissociated by 2 days. This behavior matched the experimental observation in Test 11 (Figure 3.1.37). The variation in hydrate saturation was small.

Figure 3.2.12 shows the water saturation within the core. The average saturation is plotted as the middle line. The variation in water saturation is shown as an error bar. The average saturation started to decrease from the beginning, but decreased faster after 0.4 days. Most of the hydrate dissociated by 2 days; the saturation did not change much afterwards. The variation in water saturation was significant and increased with time.

Figure 3.2.13 shows the gas saturation within the core. The average saturation is plotted as the middle line. The variation in gas saturation is shown as an error bar. The average saturation started to increase after 0.4 days at the onset of hydrate dissociation. Most of the hydrate dissociated by 2 days; the saturation did not change much afterwards. The variation in gas saturation was significant and increased with time. 
Figure 3.2.14 shows the total volume of hydrate, aqueous, gas and ice in the core. The hydrate has completely dissociated after 2 days. No ice formed in this simulation. Figure 3.2.15 shows the cumulative amount of methane production with time at the pressure in the piston after accounting for the expansion of connected and disconnected gases present. This data can be compared with the experimental data shown in Figure 3.1.35. The gas production is approximately constant after accounting for the water production. 
Figure 3.2.9. Test 11: Average Pressure throughout the Core with Time The Error Bar is the Variation in all the Grid Blocks

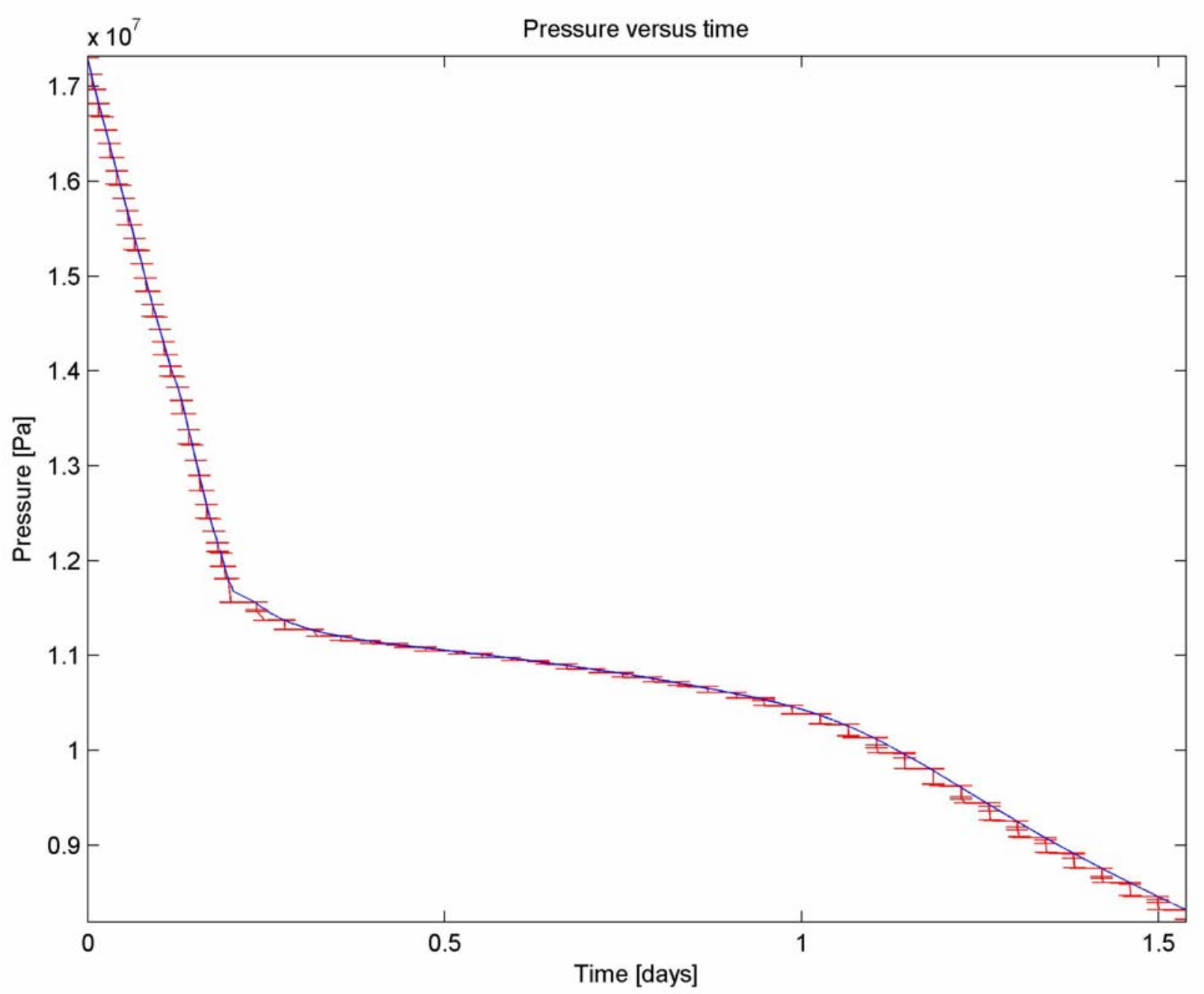


Figure 3.2.10. Test 11: Average Temperature throughout the Core with Time The Error Bar is the in all the Grid Blocks

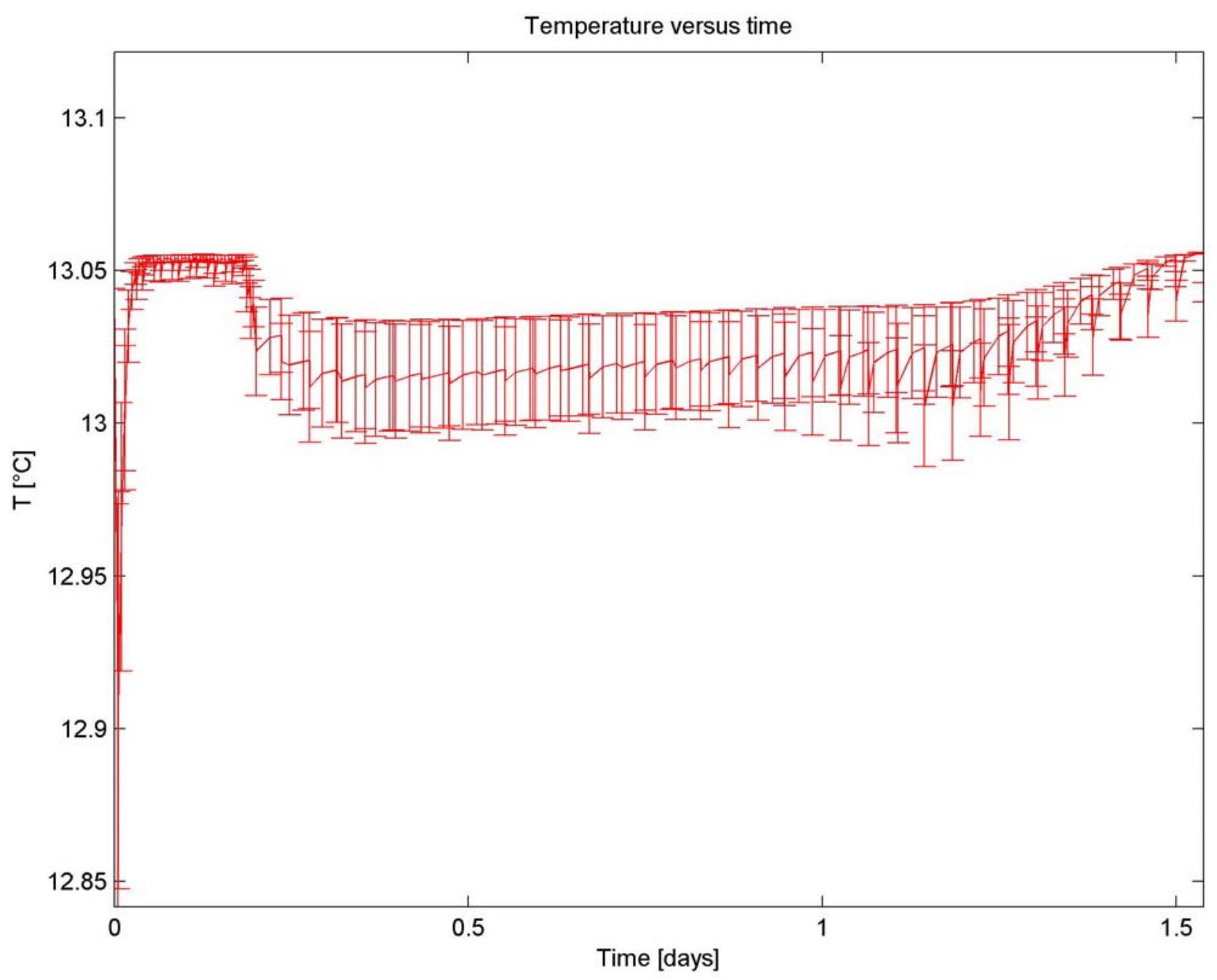


Figure 3.2.11. Test 11: Average Hydrate Saturation throughout the Core with Time The Error Bar is the Variation in all the Grid Blocks

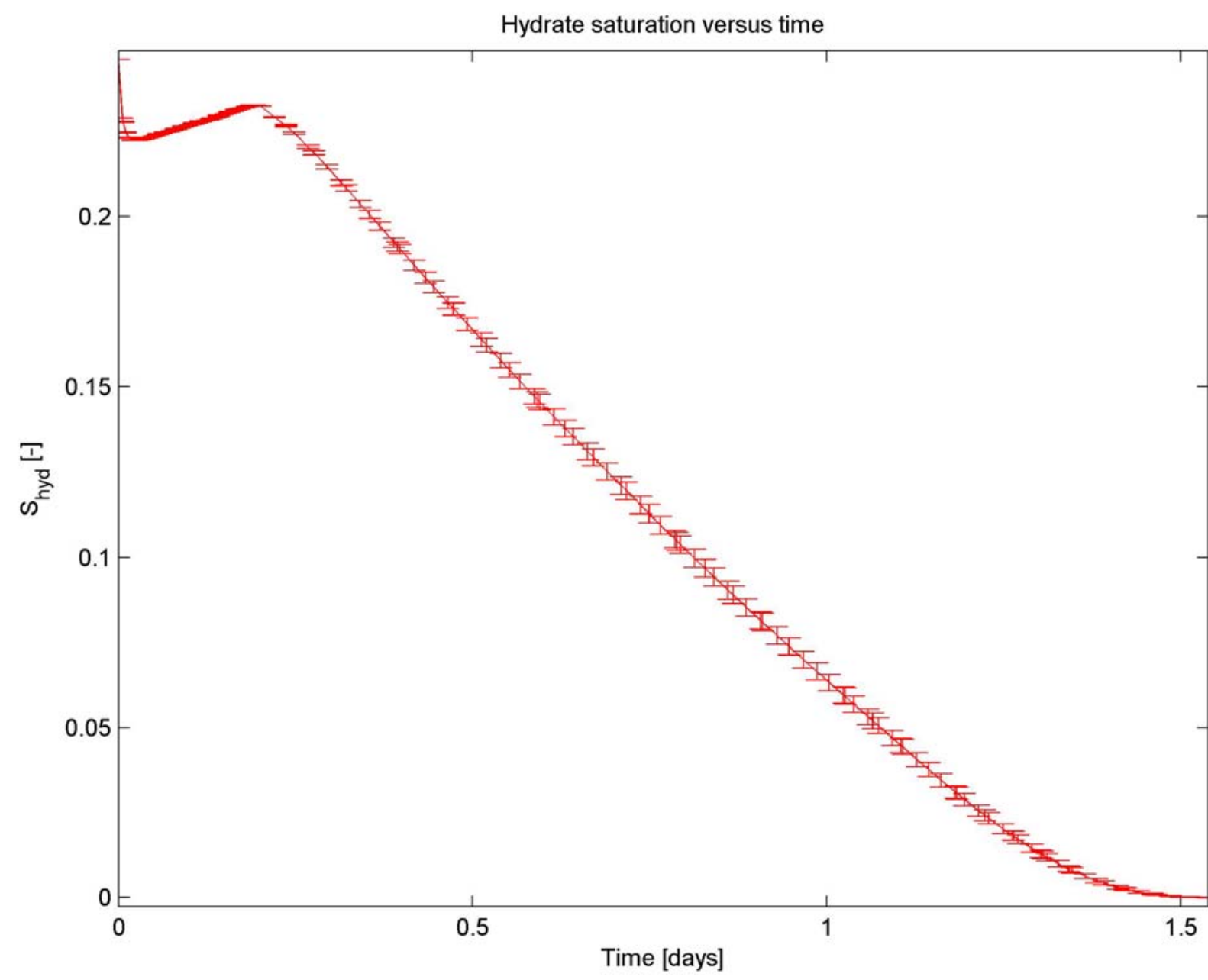


Figure 3.2.12. Test 11: Average Aqueous Saturation throughout the Core with Time The Error Bar is the Variation in all the Grid Blocks

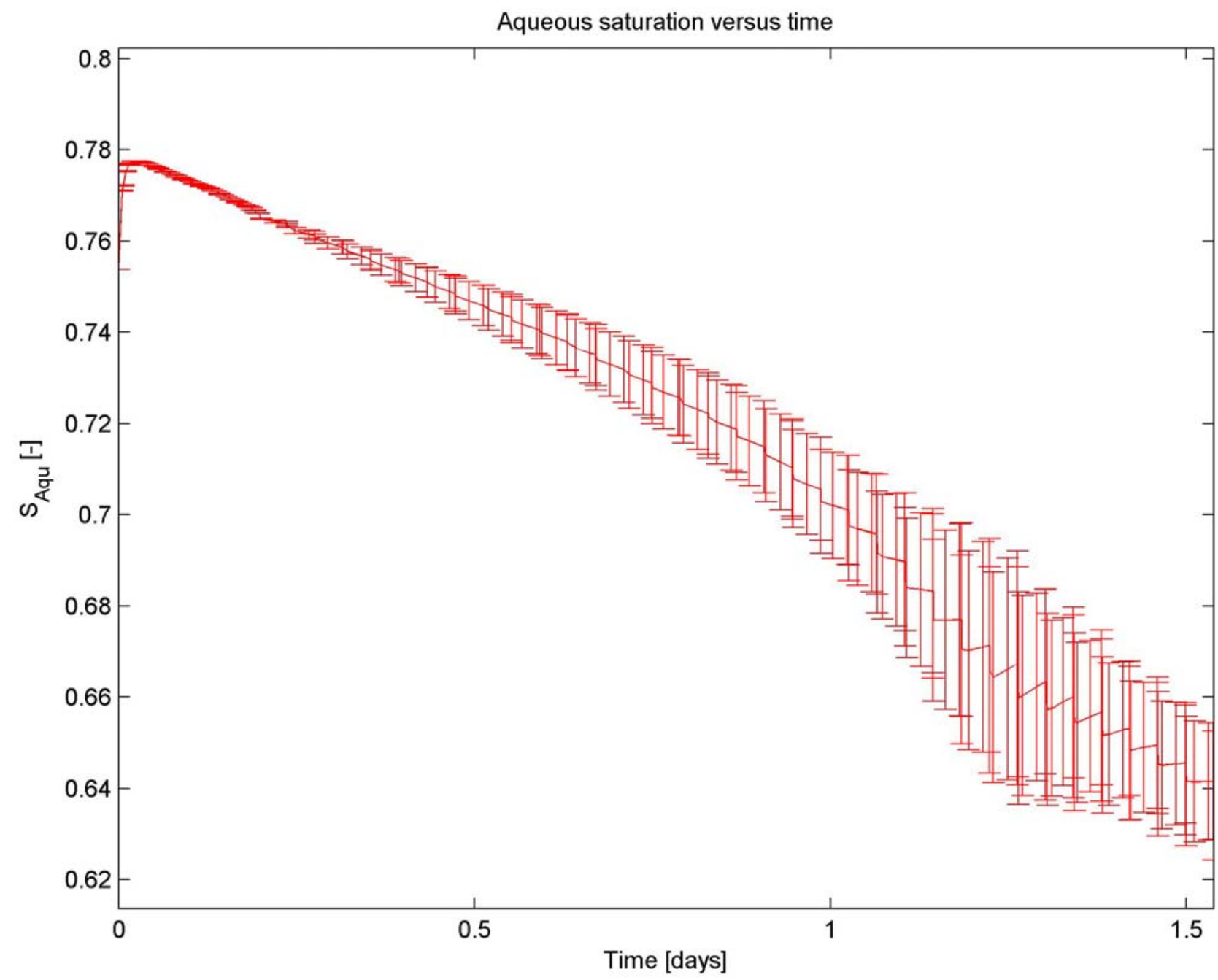


Figure 3.2.13. Test 11: Average Gas Saturation throughout the Core with Time The Error Bar is the Variation in all the Grid Blocks

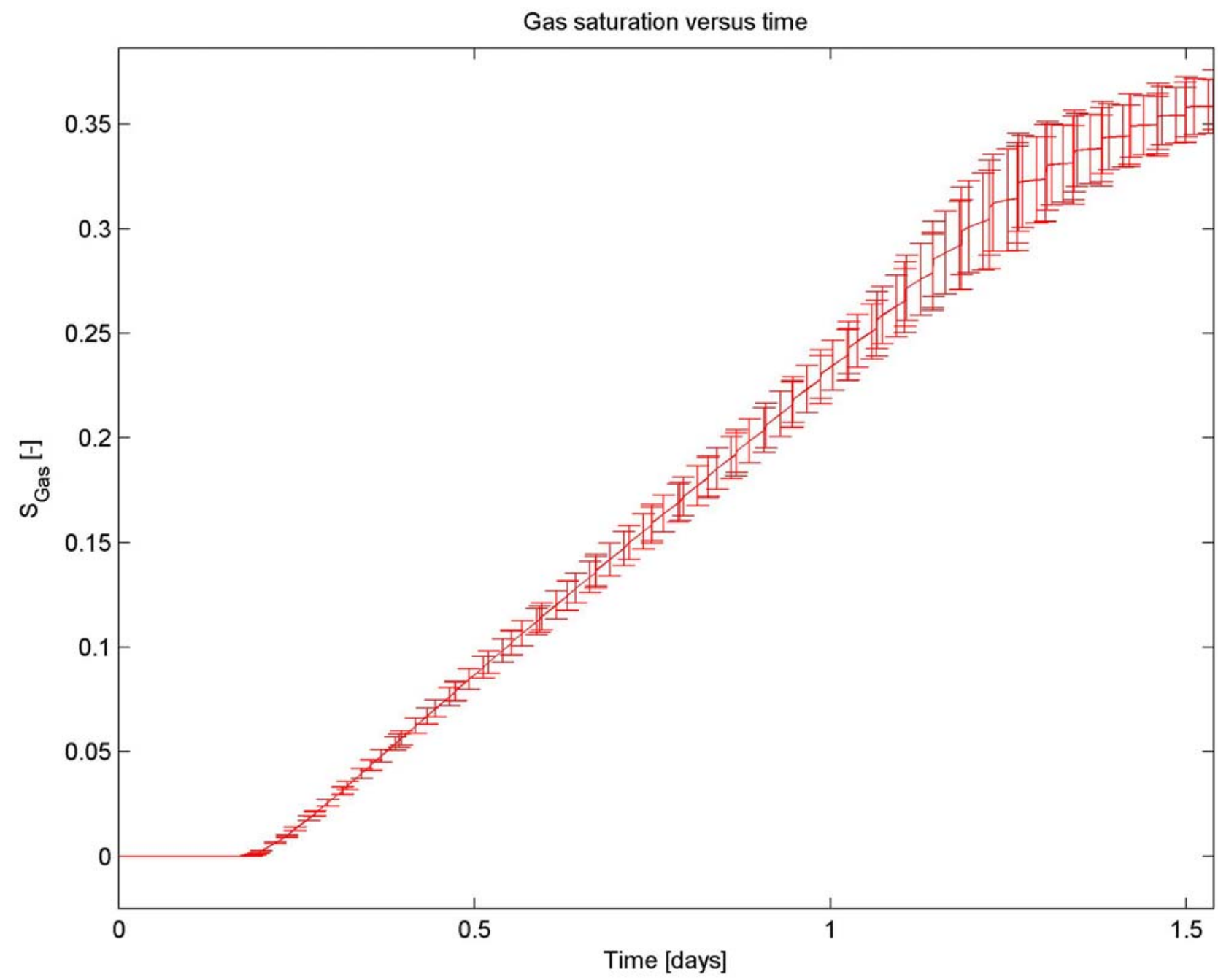


Figure 3.2.14. Test 11: Total Volume of Gas, Hydrate, Aqueous and Ice in the Core with Time The Error Bar is the Variation in all the Grid Blocks

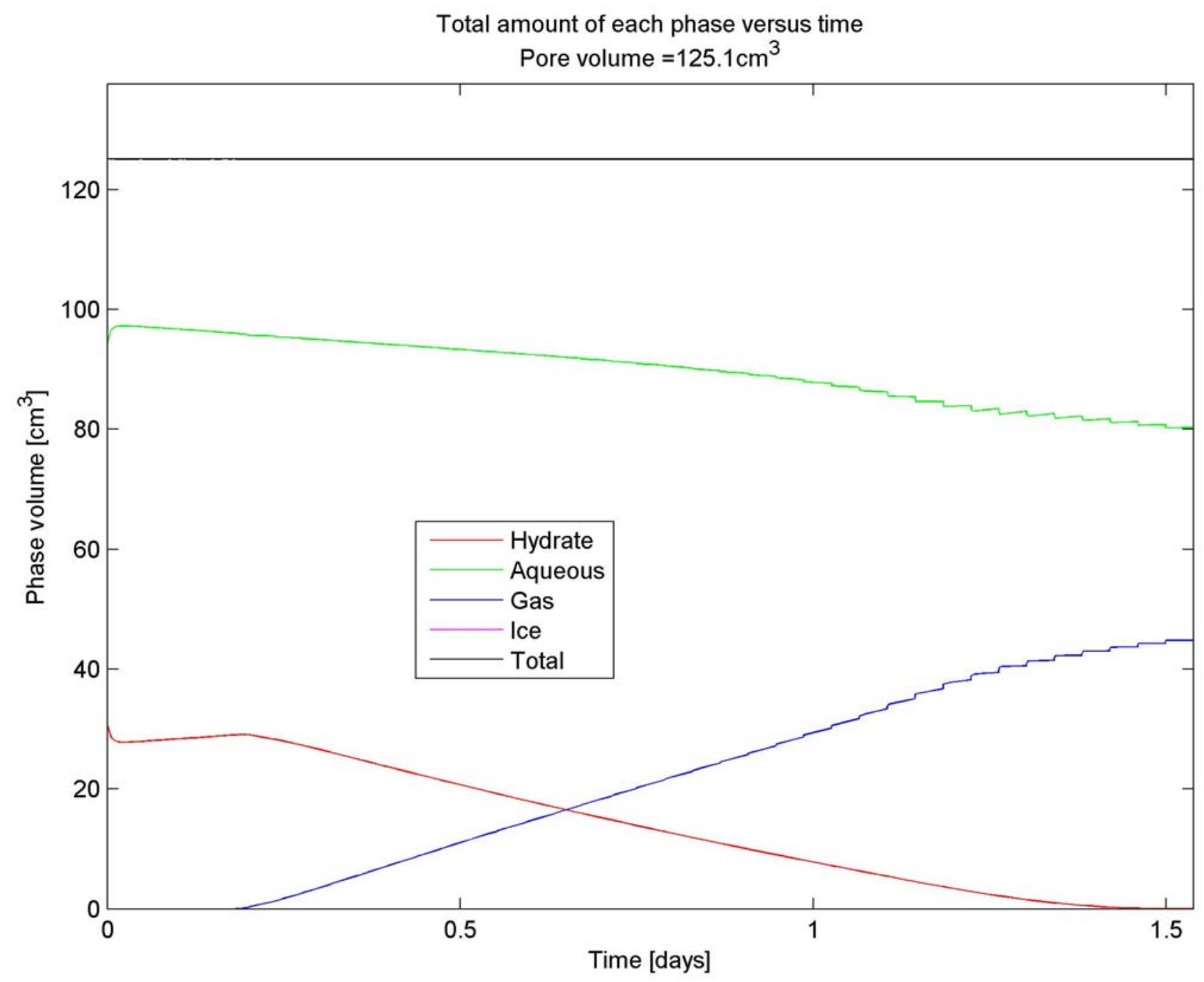


Figure 3.2.15. Test 11: Cumulative Volume of Methane Released over a Period of Time

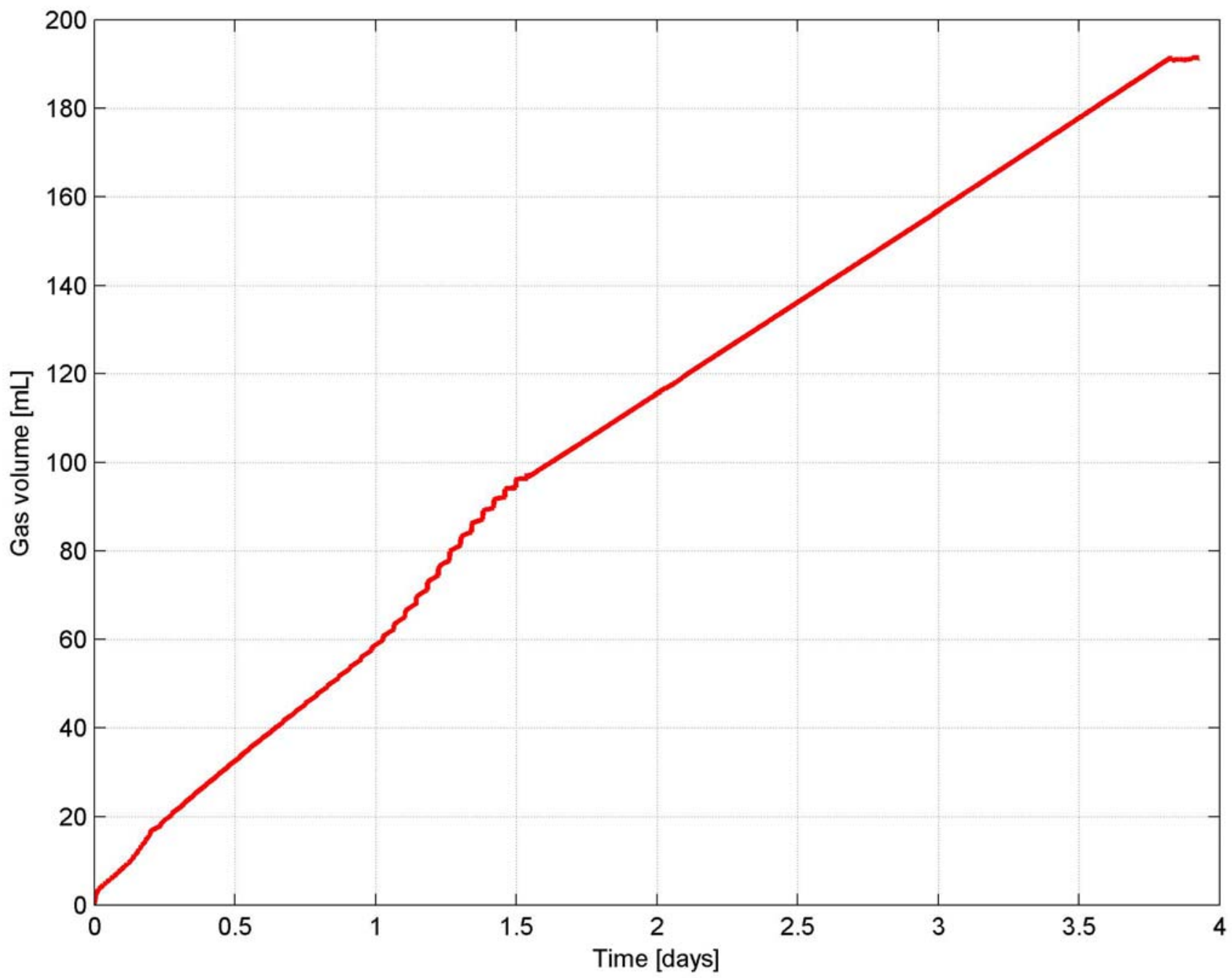




\subsubsection{Comparison of Model Output with Test 12}

Figures 3.2.16 through 3.2.20 show the simulation results for Test 12. In this test the depressurization was conducted at a much slower rate than Test 10 , similar to Test 11 . The final pressure was a little higher in this case. The initial connected gas volume was $27 \mathrm{ml}$ and disconnected volume was $16.3 \mathrm{ml}$. Figure 3.2.16 shows the variation of the average pressure in the core with time. What is shown as an error bar is the standard deviation in pressure among different grid blocks. The average pressure tracked the well pressure. The pressure fell sharply before hydrate dissociation indicating expansion of gas and expulsion of brine. The pressure fell slowly during hydrate dissociation indicating release of gas to fill up the volume created by the withdrawal of the piston. The pressure fell at a faster rate after the hydrate dissociation due the expansion of gas, again. The error bars are small after hydrate dissociation, indicating small pressure drops within the core.

Figure 3.2.17 shows the variation of temperature within the core. The average temperature is shown as the middle line. The variation in the temperature is shown as an error bar. The temperature was more or less constant before 0.4 days, fell during the hydrate depressurization, and eventually equilibrated to boundary temperature. This behavior matched the experimental observation in Test 12 (Figure 3.1.41). During the hydrate depressurization the latent heat was extracted from the vicinity of the hydrate which lowered the temperature.

Figure 3.2.18 shows the hydrate saturation within the core. The average saturation is plotted as the middle line. The variation in hydrate saturation is shown as an error bar. The average saturation started to decrease after 0.4 days. Most of the hydrate dissociated by 2 days. This behavior matched the experimental observation in Test 12 (Figure 4.1.43). Again the variation in hydrate saturation was small.

Figure 3.2.19 shows the water saturation within the core. The average saturation is plotted as the middle line. The variation in water saturation is shown as an error bar. The average saturation started to decrease from the beginning, but decreased faster after 0.4 days. Most of the hydrate dissociated by 2 days; the saturation did not change much afterwards. The variation in water saturation was significant and increased with time.

Figure 3.2.20 shows the gas saturation within the core. The average saturation is plotted as the middle line. The variation in gas saturation is shown as an error bar. The average saturation started to increase after 0.4 days at the onset of hydrate dissociation. Most of the hydrate dissociated by 2 days; the saturation did not change much afterwards. The variation in gas saturation was significant and increased with time. 
Figure 3.2.21 shows the total volume of hydrate, aqueous, gas and ice in the core. The hydrate was completely dissociated after 2 days. No ice formed in this simulation. Figure 3.2.22 shows the cumulative amount of methane production with time at the pressure in the piston after accounting for the expansion of connected and disconnected gases present. This data can be compared with the experimental data shown in Figure 3.1.41. The gas production is approximately constant after accounting for the water production.

Test 12 was selected for further kinetic rate variation simulations. The sensitivity of these simulations to the kinetic rate constant used is shown in Figures 3.2.23-3.2.28 for the simulations of Test 12. The only parameter changed was the kinetic rate constant; it was increased by a factor of 3 . The rate constant was $4.1 \mathrm{~kg} \mathrm{~m}-2 \mathrm{~Pa}-1 \mathrm{~s}-1$ for all the simulations reported so far. The rate constant for Figures 3.2.23 - 3.2.28 is $12 \mathrm{~kg} \mathrm{~m}-2 \mathrm{~Pa}-1 \mathrm{~s}-1$. Figure 3.2.23 shows the pressure distribution; it is very similar to Figure 3.2.16. Figure 3.2.24 shows the temperature distribution. The temperature falls more in this case compared to that shown in Figure 3.2.17. Higher kinetic constant increases the dissociation rate, thus higher is the heat removal and lower is the temperature. Figure 3.2.25 shows the hydrate saturation; it falls faster compared to that shown in Figure 3.2.18. This leads to increases in water saturation shown in Figure 3.2.26 compared to that in Figure 3.2.19. The gas saturation is lower in Figure 3.2.27 compared to Figure 3.2.20. Gas flows out lowering the in situ saturation. The volumes of the three phases in the core are shown in Figure 3.2.28. Thus this dissociation is kinetically controlled. 
Figure 3.2.16. Test 12: Average Pressure throughout the Core with Time The Error Bar is the Variation of Pressure in all the Grid Blocks

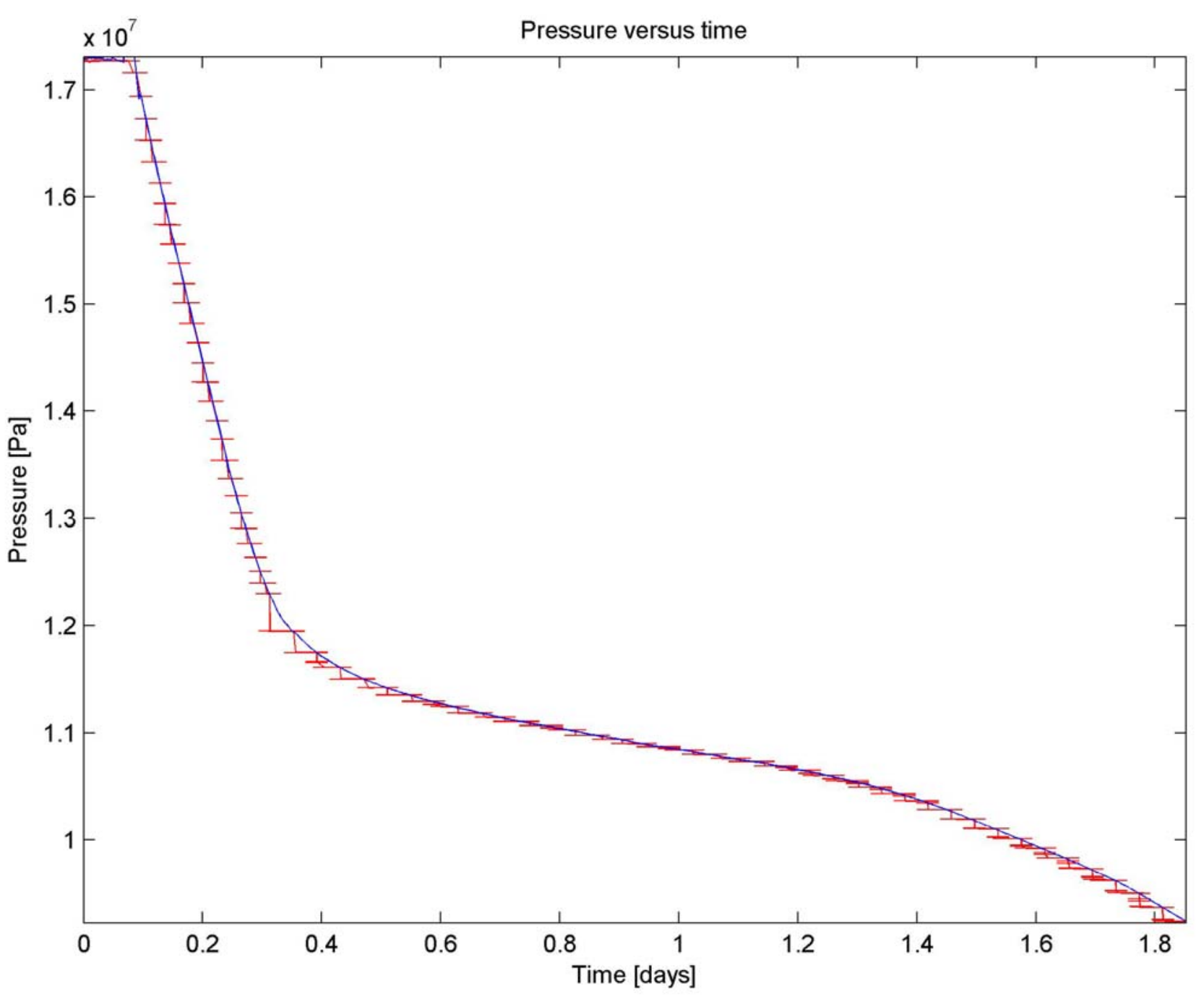


Figure 3.2.17. Test 12: Average Temperature throughout the Core with Time The Error Bar is the Variation in all the Grid Blocks

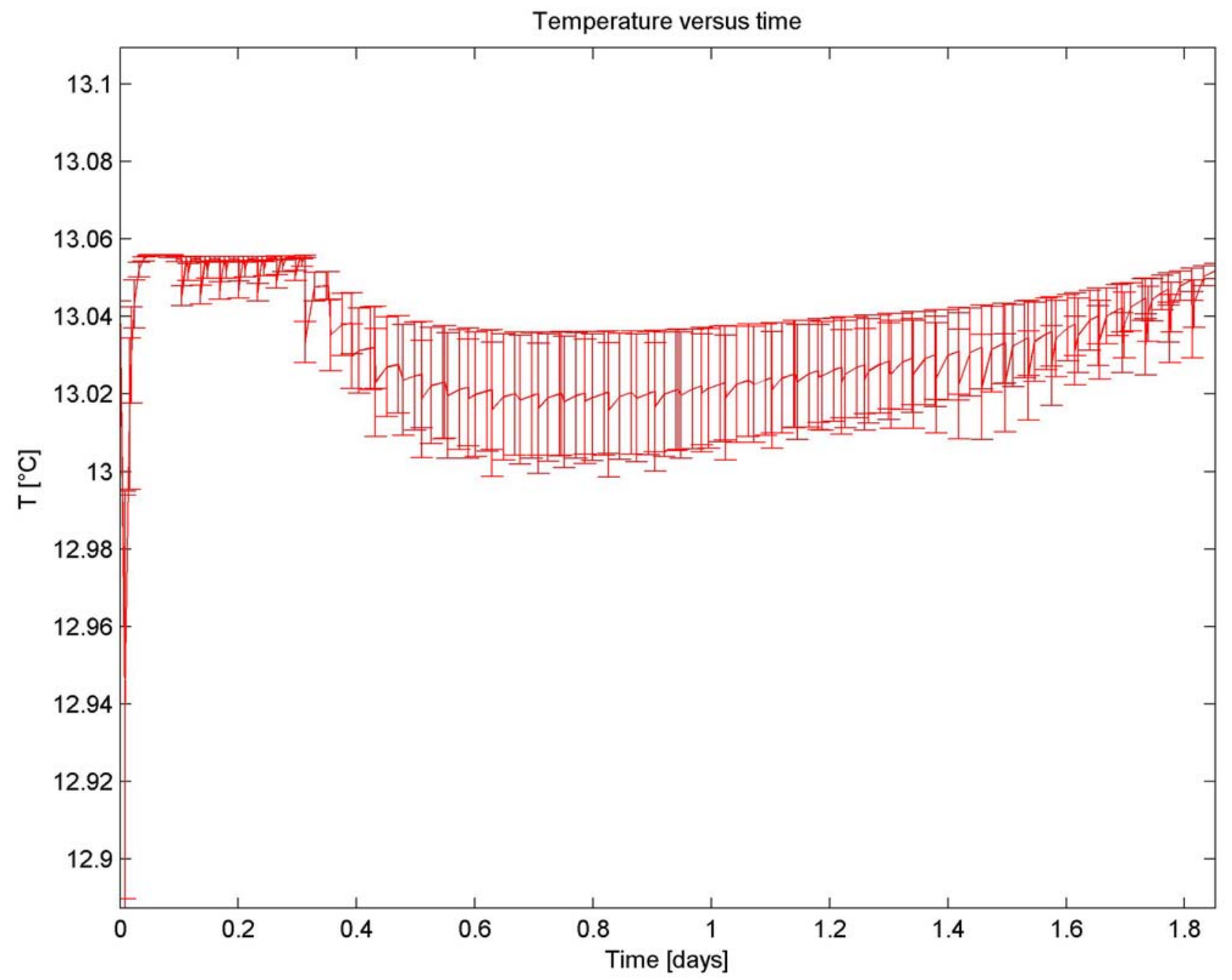


Figure 3.2.18. Test 12: Average Hydrate Saturation throughout the Core with Time The Error bar is the Variation in all the Grid Blocks

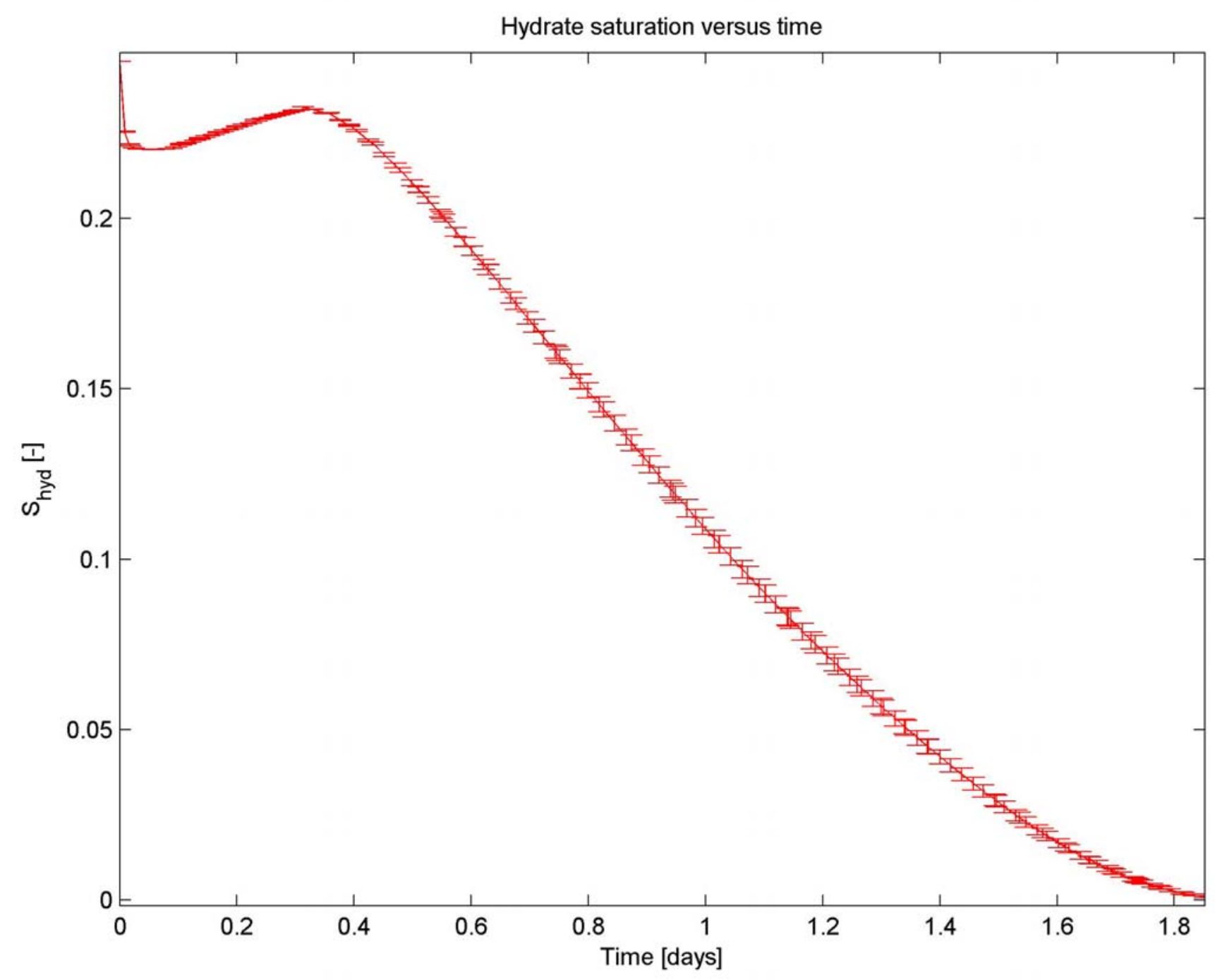


Figure 3.2.19. Test 12: Average Aqueous Saturation throughout the Core with Time The Error Bar is the Variation in all the Grid Blocks

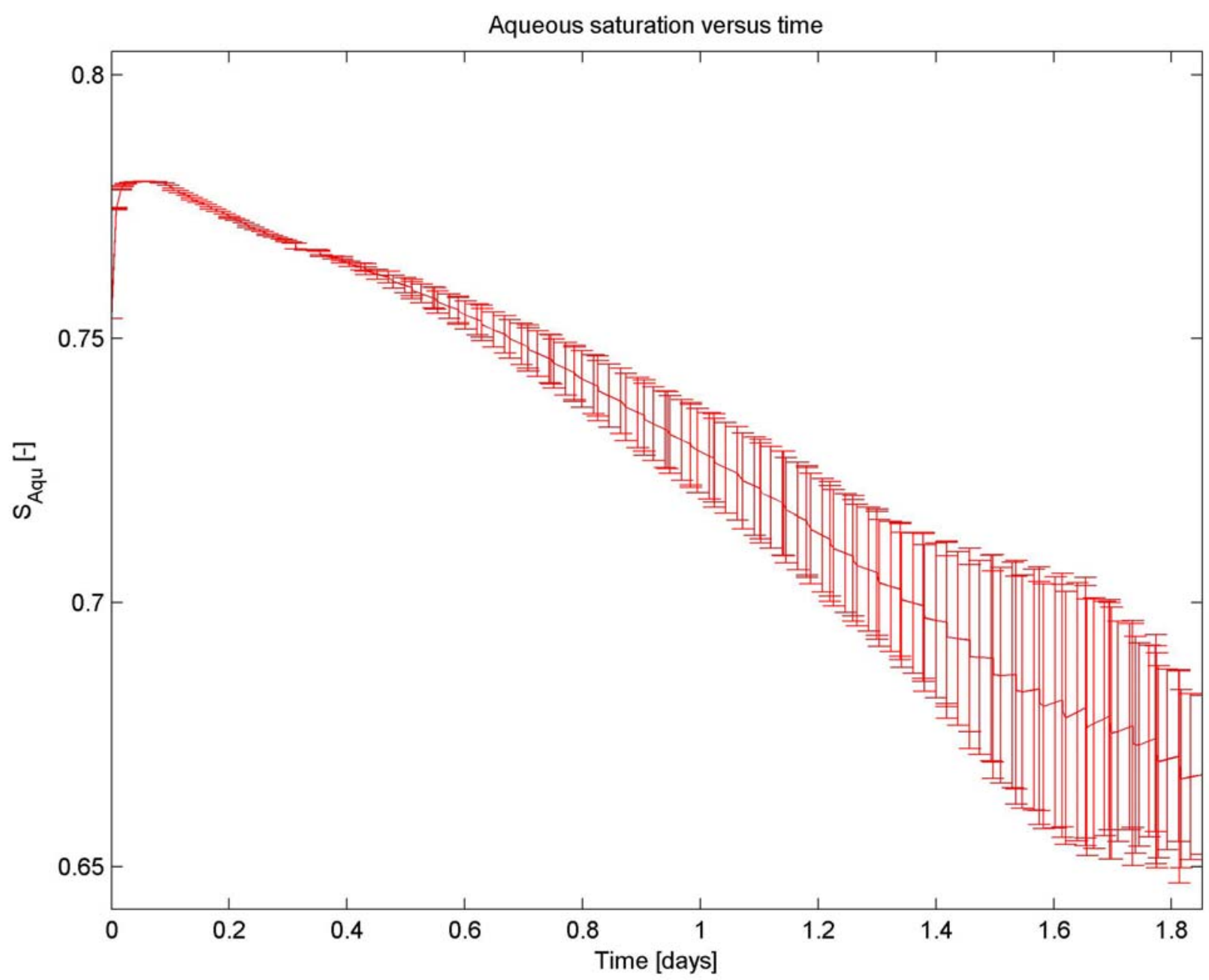


Figure 3.2.20. Test 12: Average Gas Saturation throughout the Core with Time The Error Bar is the Variation in all the Grid Blocks

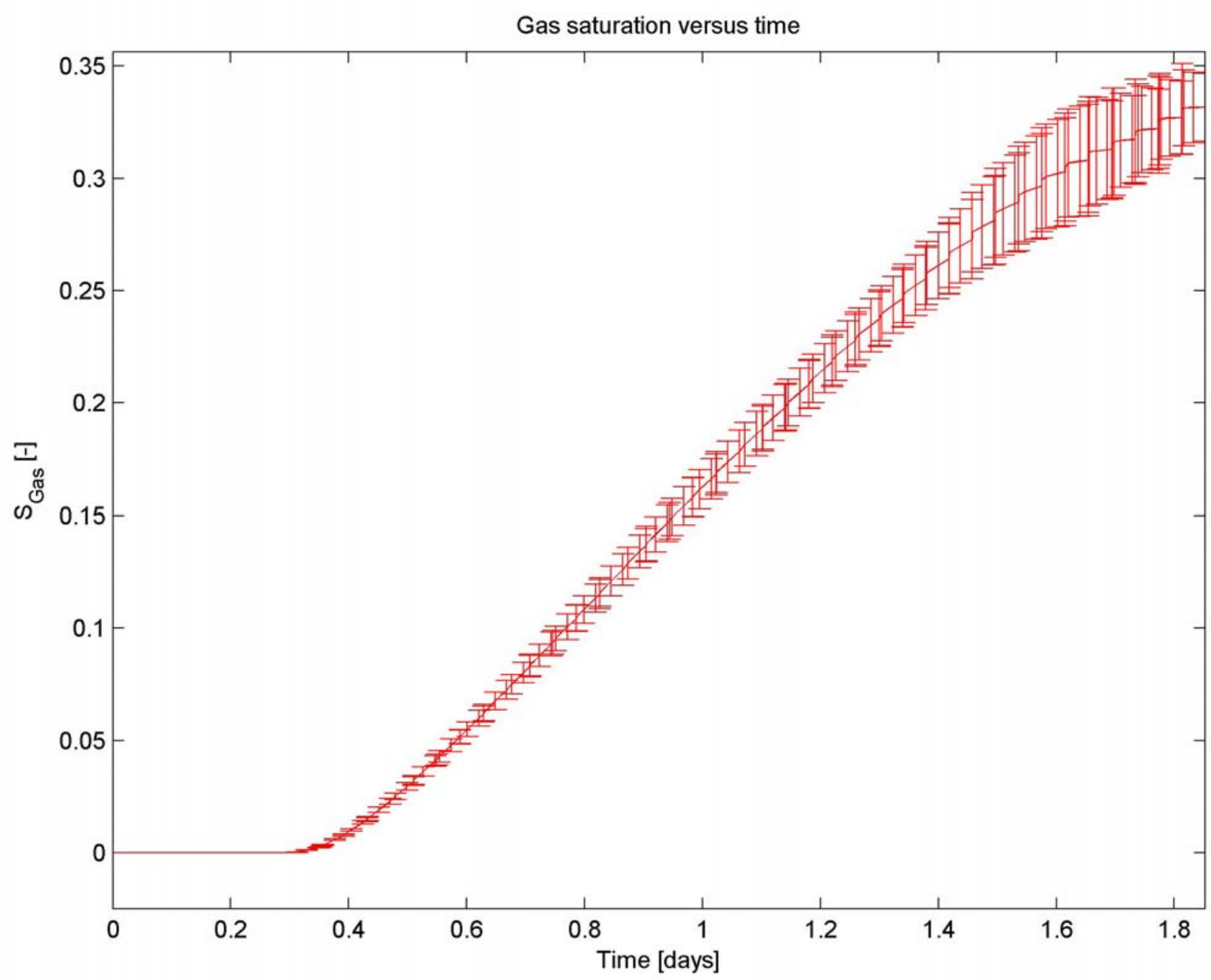


Figure 3.2.21. Test 12: Total Volume of Gas, Hydrate, Aqueous and Ice in the Core with Time The Error Bar is the Variation in all the Grid Blocks

Total amount of each phase versus time Pore volume $=125.1 \mathrm{~cm}^{3}$

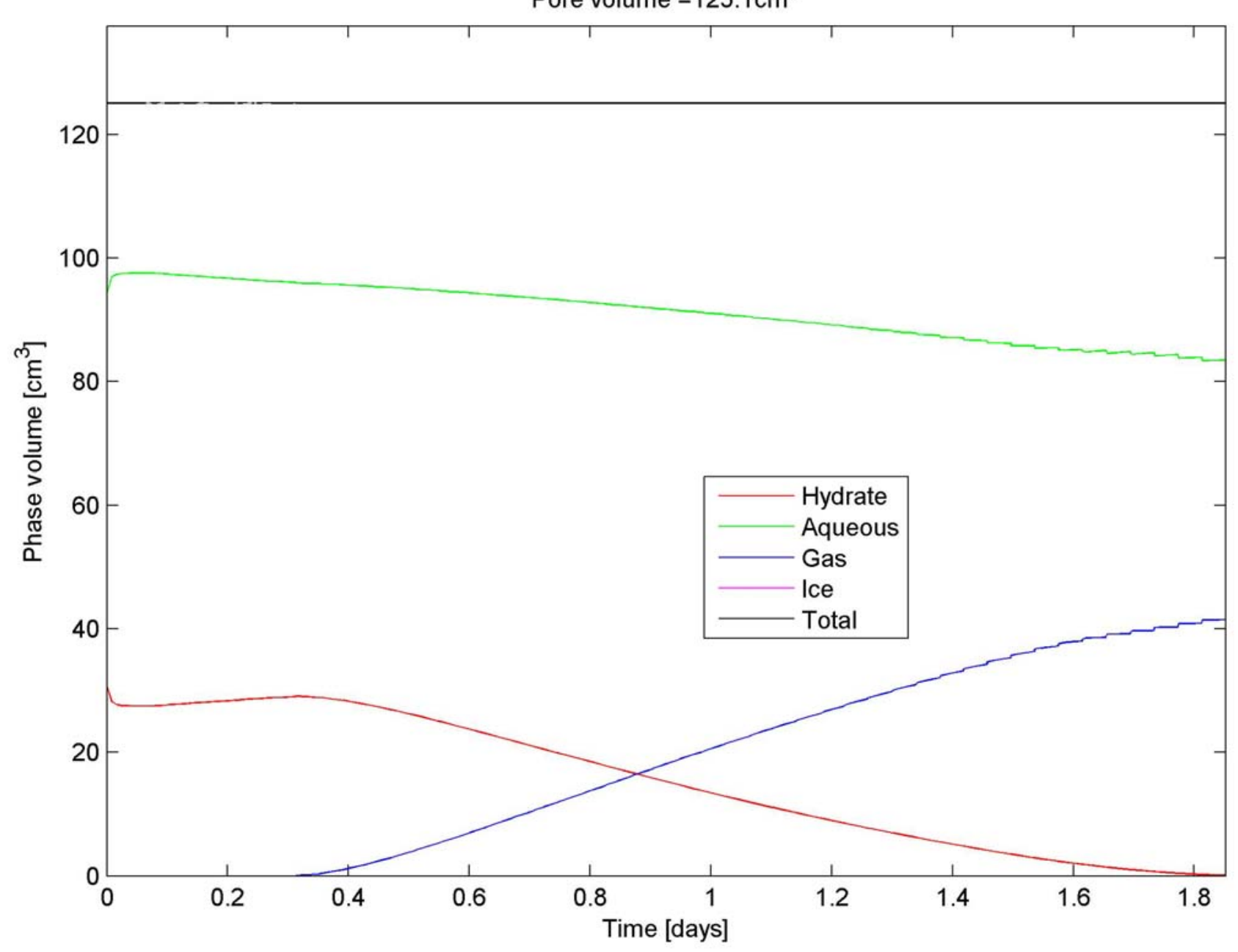


Figure 3.2.22. Test 12: Cumulative Volume of Methane Released over a Period of Time

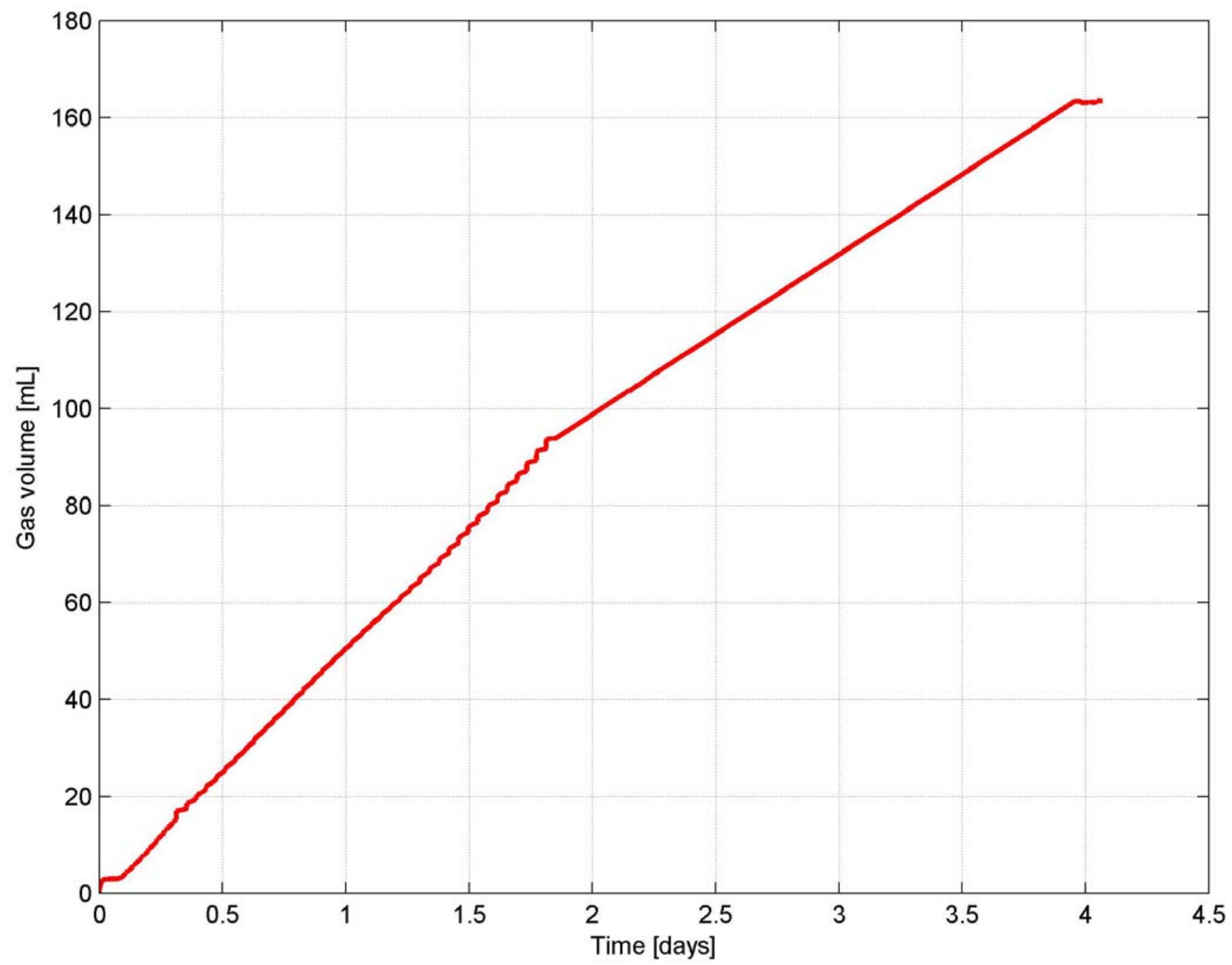


Figure 3.2.23. Test 12: Average Pressure throughout the Core with Time The Error Bar is the Variation in all the Grid Blocks

(Kinetic rate constant is 3 times base case)

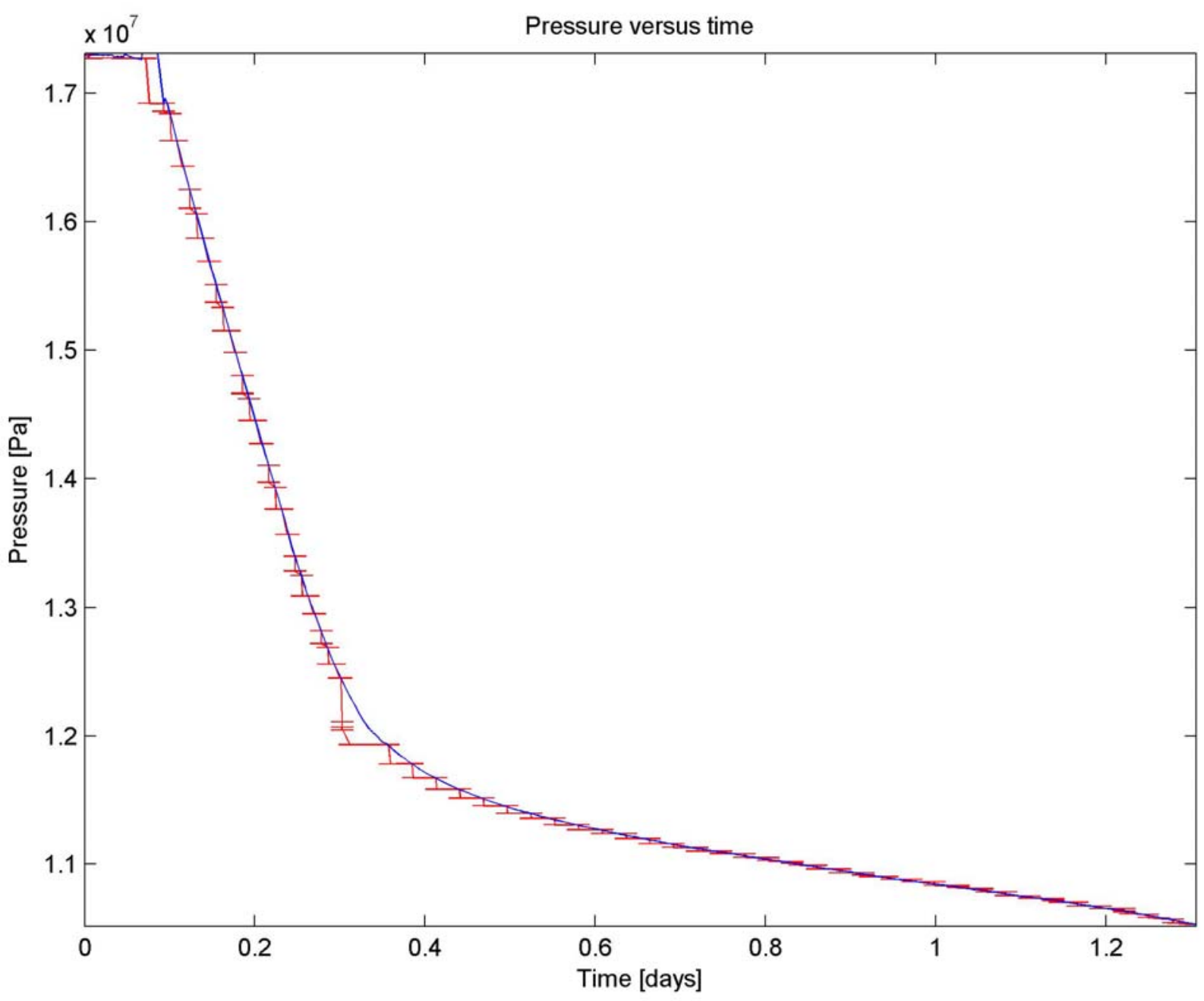


Figure 3.2.24. Test 12: Average Temperature throughout the Core with Time The Error Bar is the in all the Grid Blocks

(Kinetic rate constant is 3 times base case)

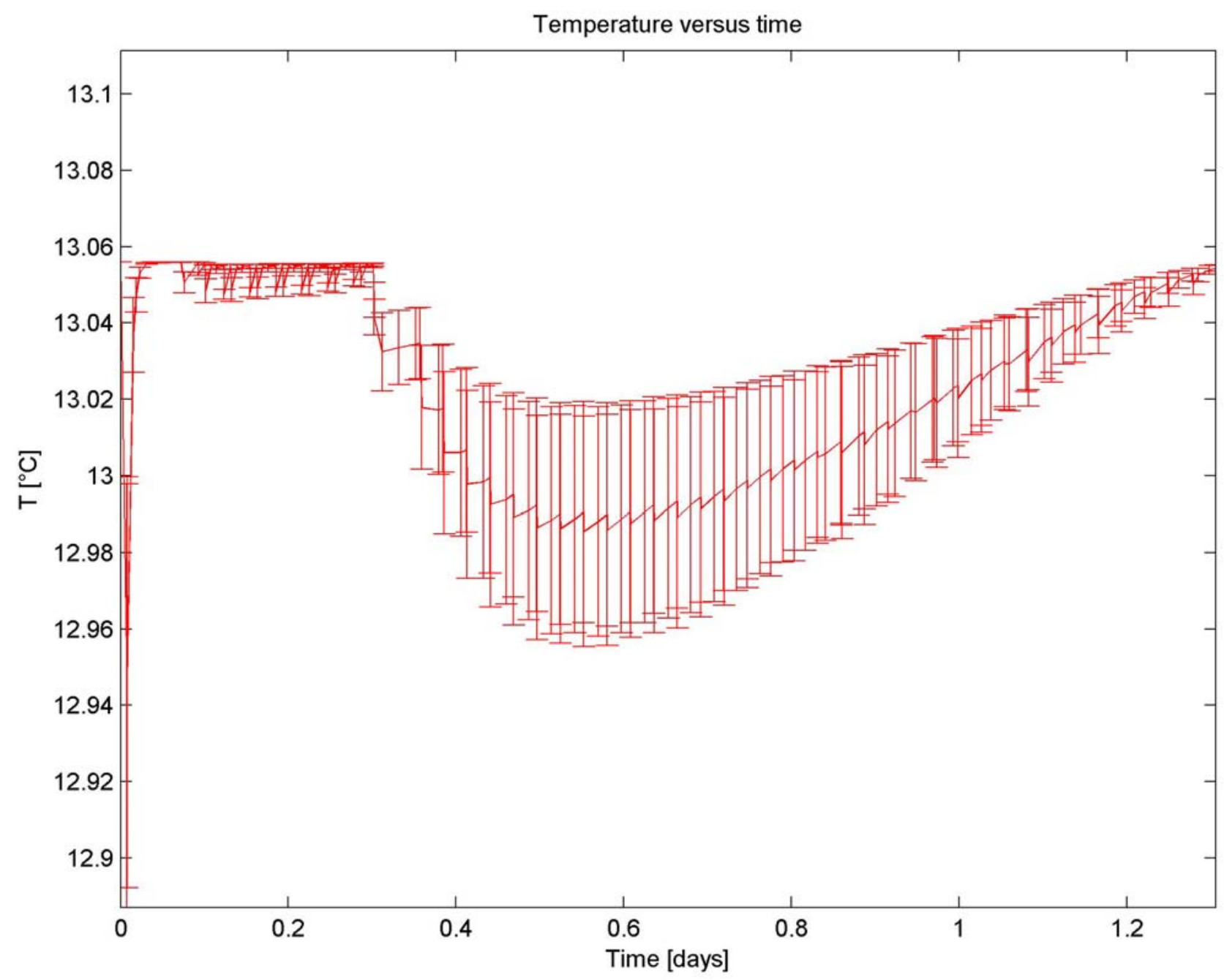


Figure 3.2.25. Test 12: Average Hydrate Saturation throughout the Core with Time The Error Bar is the Variation in all the Grid Blocks

(Kinetic rate constant is 3 times base case)

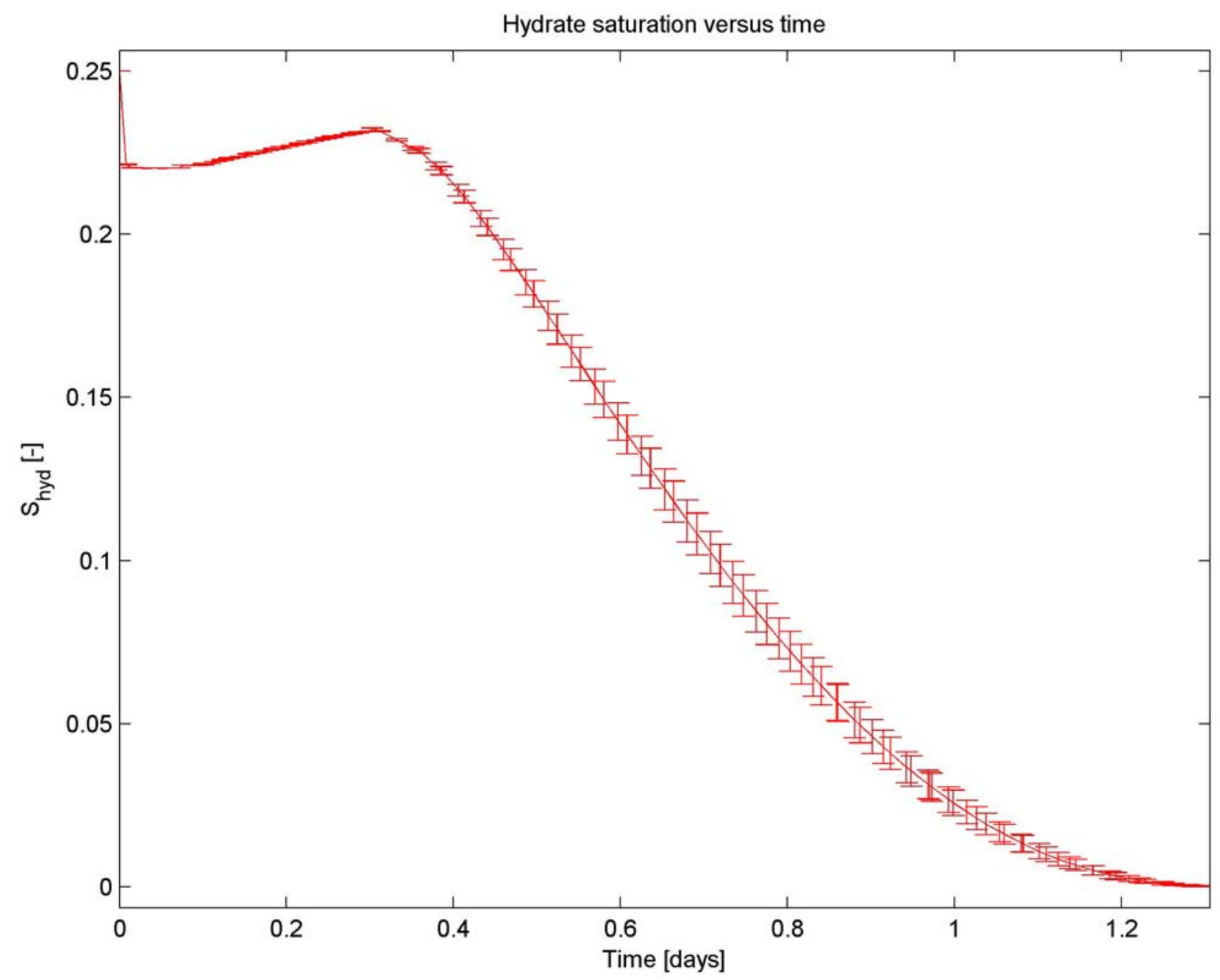


Figure 3.2.26. Test 12: Average Aqueous Saturation throughout the Core with Time The Error Bar is the Variation in all the Grid Blocks

(Kinetic rate constant is 3 times base case)

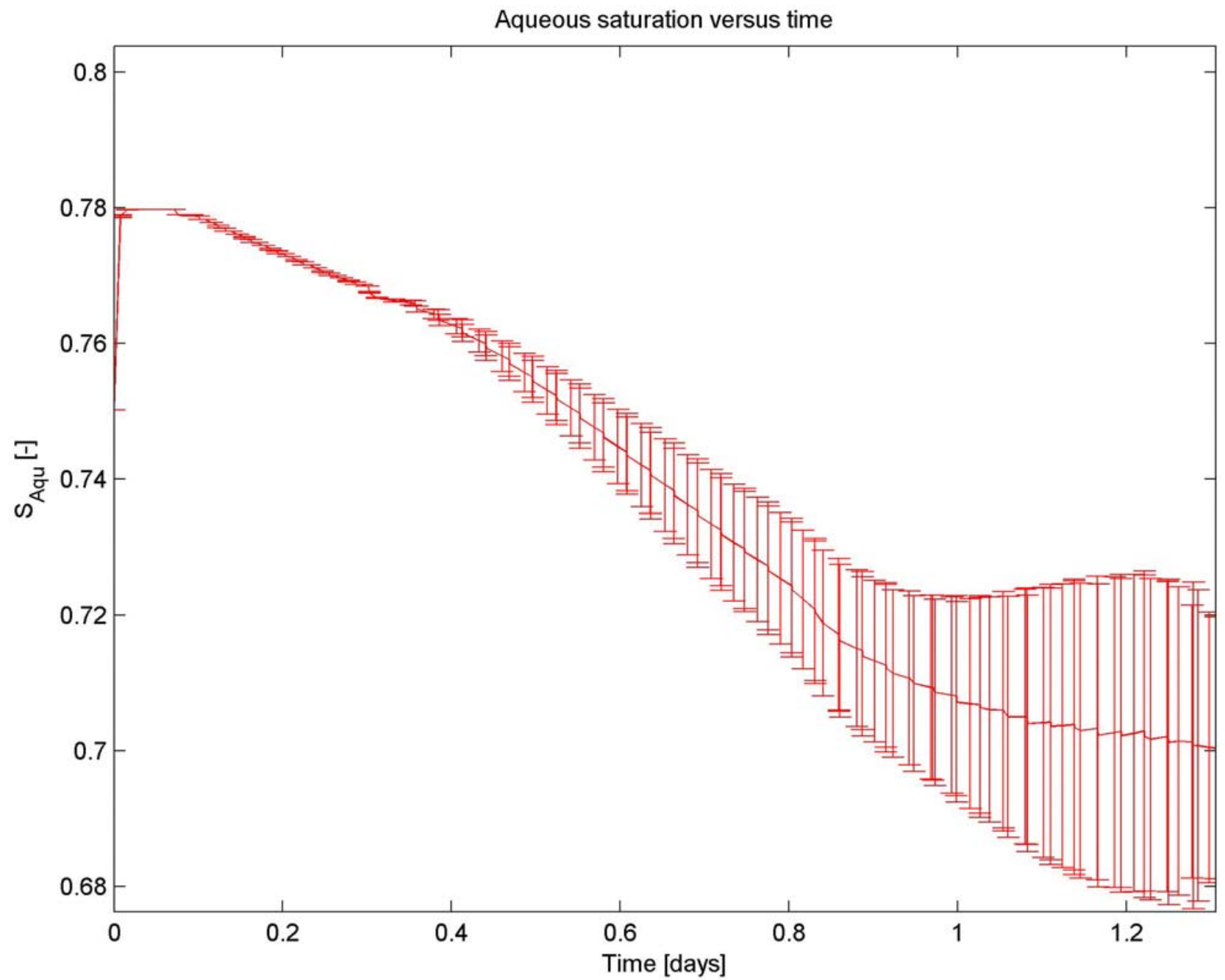


Figure 3.2.27. Test 12: Average Gas Saturation throughout the Core with Time The Error Bar is the Variation in all the Grid Blocks

(Kinetic rate constant is 3 times base case)

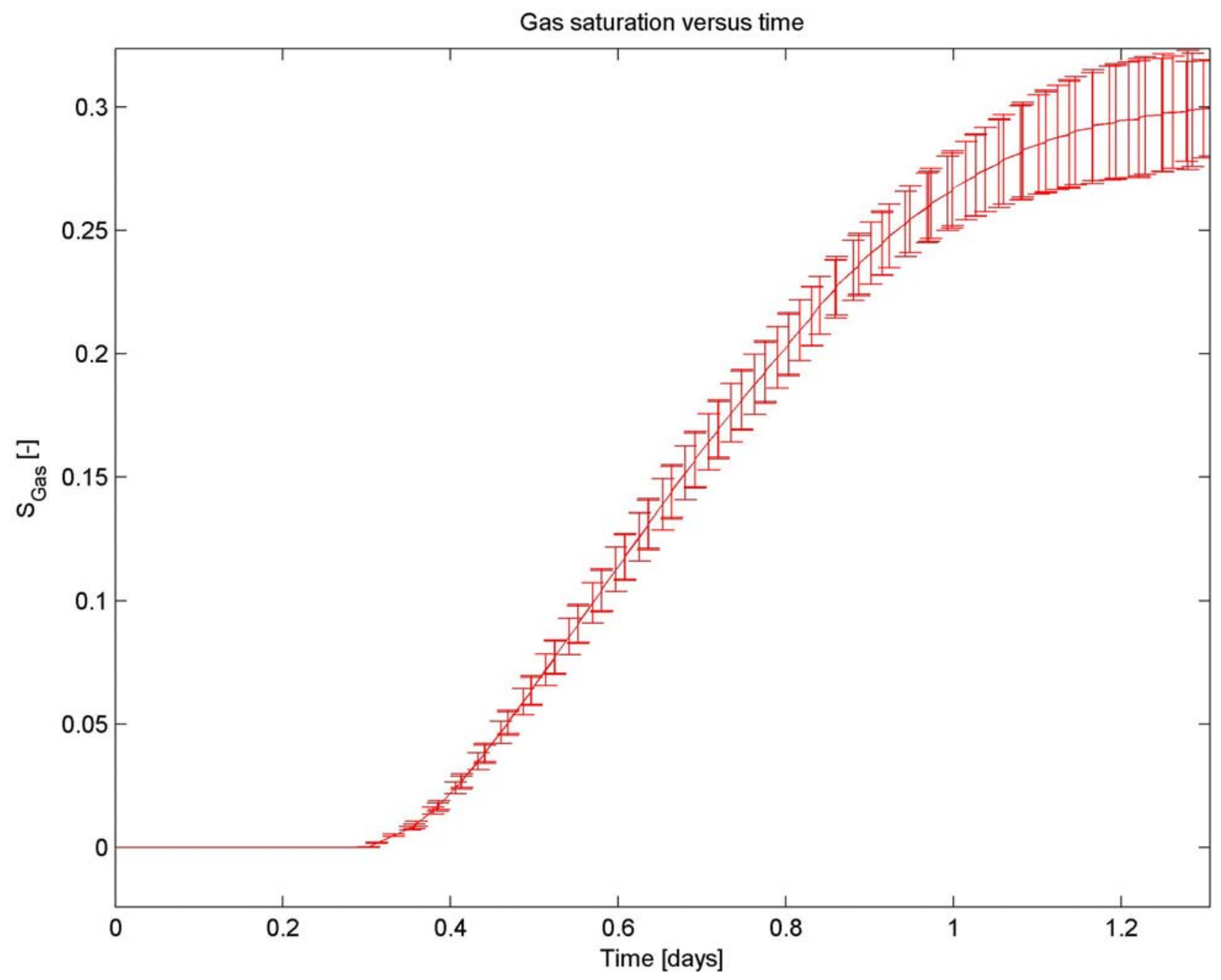


Figure 3.2.28. Test 12: Total Volume of Gas, Hydrate, Aqueous and Ice in the Core with Time The Error Bar is the Variation in all the Grid Blocks

(Kinetic rate constant is 3 times base case)

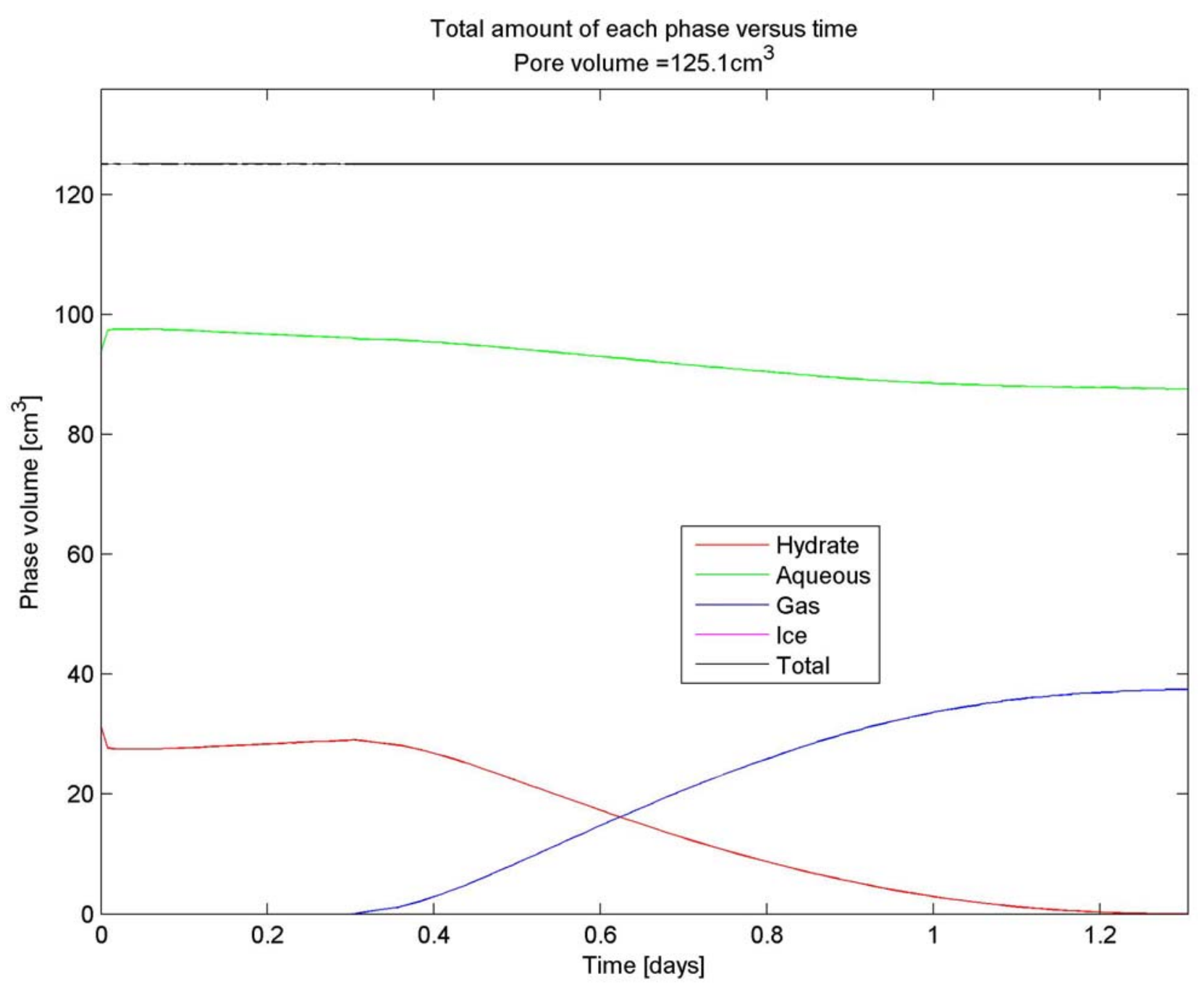




\subsubsection{Comparison of Model Output to Test 13}

Figures 3.2.29 though 3.2.35 show the simulation results for Test 13. This test was conducted in a core that mimics the unconsolidated nature of the Gulf of Mexico sediments. The hydrate saturation was much lower here compared to the last three tests $(\sim 12.4 \mathrm{ml})$. The temperature for dissociation was much lower here, about $33^{\circ} \mathrm{F}$ (compared to $55^{\circ} \mathrm{F}$ for the others). The final pressure was a little higher in this case. The initial connected gas volume was $16.8 \mathrm{ml}$ and disconnected volume was $10.2 \mathrm{ml}$.

Figure 3.2.29 shows the variation of the average pressure in the core with time. What is shown as an error bar is the standard deviation in pressure among different grid blocks. The average pressure tracked the well pressure. The pressure fell sharply before hydrate dissociation indicating expansion of gas and expulsion of brine. The pressure fell slowly during hydrate dissociation indicating release of gas to fill up the volume created by the withdrawal of the piston. The pressure fell at a faster rate after the hydrate dissociation due the expansion of gas, again. The error bars are small after hydrate dissociation, indicating small pressure drops within the core.

Figure 3.2.30 shows the variation of temperature within the core. The average temperature is shown as the middle line. The variation in the temperature is shown as an error bar. The temperature was more or less constant before 0.2 days, fell during the hydrate depressurization, and eventually equilibrated to boundary temperature. The temperature drops were very small here for detection; this behavior matched the experimental observation in Test 13.

Figure 3.2.31 shows the hydrate saturation within the core. The average saturation is plotted as the middle line. The variation in hydrate saturation is shown as an error bar. The average saturation started to decrease after 0.2 days. Most of the hydrate dissociated by 0.6 days. Again the variation in hydrate saturation was small.

Figure 3.2.32 shows the water saturation within the core. The average saturation is plotted as the middle line. The variation in water saturation is shown as an error bar. The average saturation started to decrease from the beginning, but decreased faster after 0.2 days. Most of the hydrate dissociated by 0.6 days; the saturation did not change much afterwards. The variation in water saturation was significant and increased with time.

Figure 3.2.33 shows the gas saturation within the core. The average saturation is plotted as the middle line. The variation in gas saturation is shown as an error bar. The average saturation started to increase after 0.2 days at the onset of hydrate dissociation. Most of the hydrate dissociated by 0.6 days; the saturation did not change much afterwards. The variation in gas saturation was significant and increased 
with time.

Figure 3.2.34 shows the total volume of hydrate, aqueous, gas and ice in the core. The hydrate was completely dissociated after 0.6 days. No ice formed in this simulation. Figure 3.2.35 shows the cumulative amount of methane production with time at the pressure in the piston after accounting for the expansion of connected and disconnected gases present. This data can be compared with the experimental data shown in Figure 3.1.47. The gas production is approximately constant after accounting for the water production. 
Figure 3.2.29. Test 13: Average Pressure throughout the Core with Time The Error Bar is the Variation in all the Grid Blocks

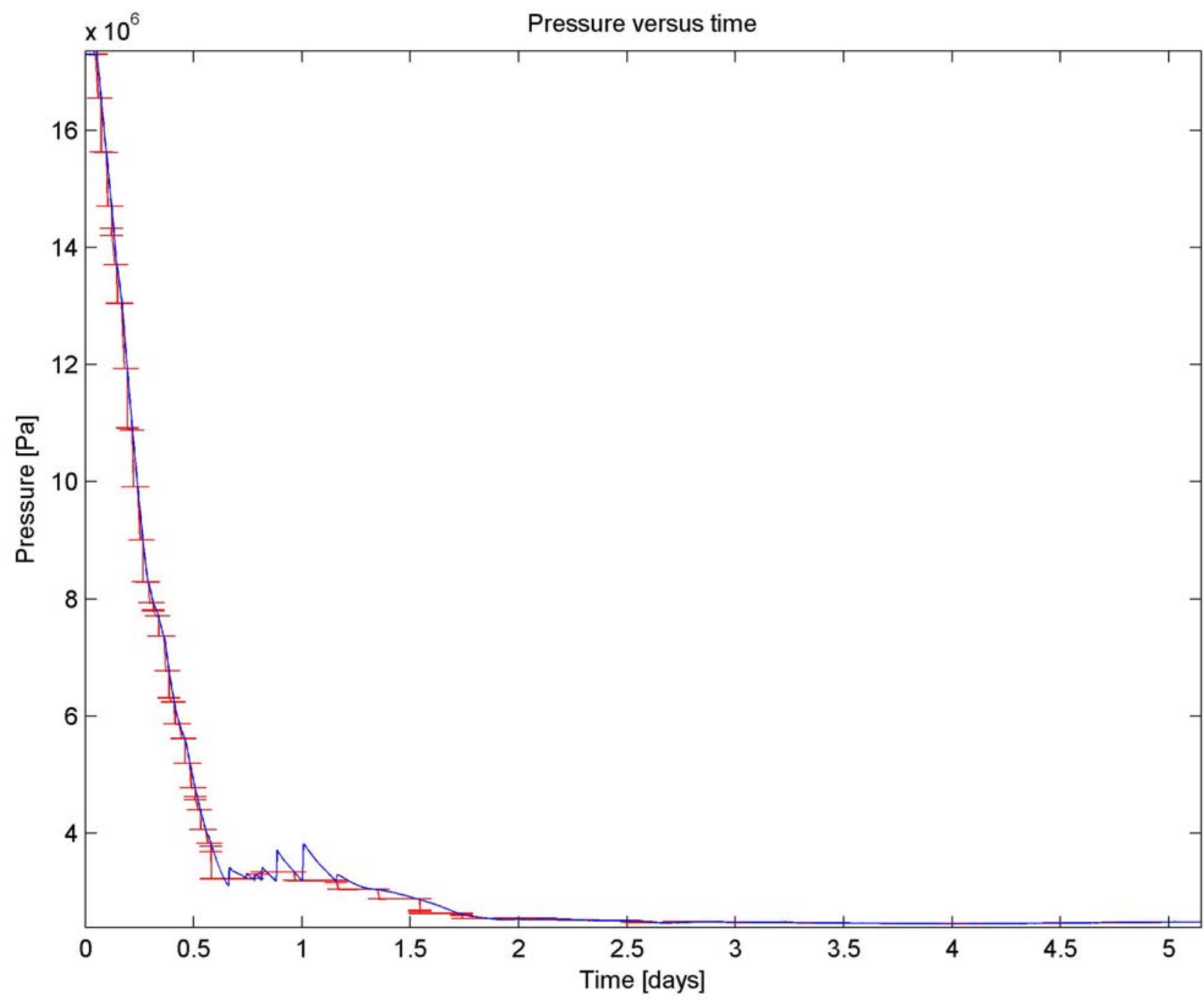


Figure 3.2.30. Test 13: Average Temperature throughout the Core with Time The Error Bar is the in all the Grid Blocks

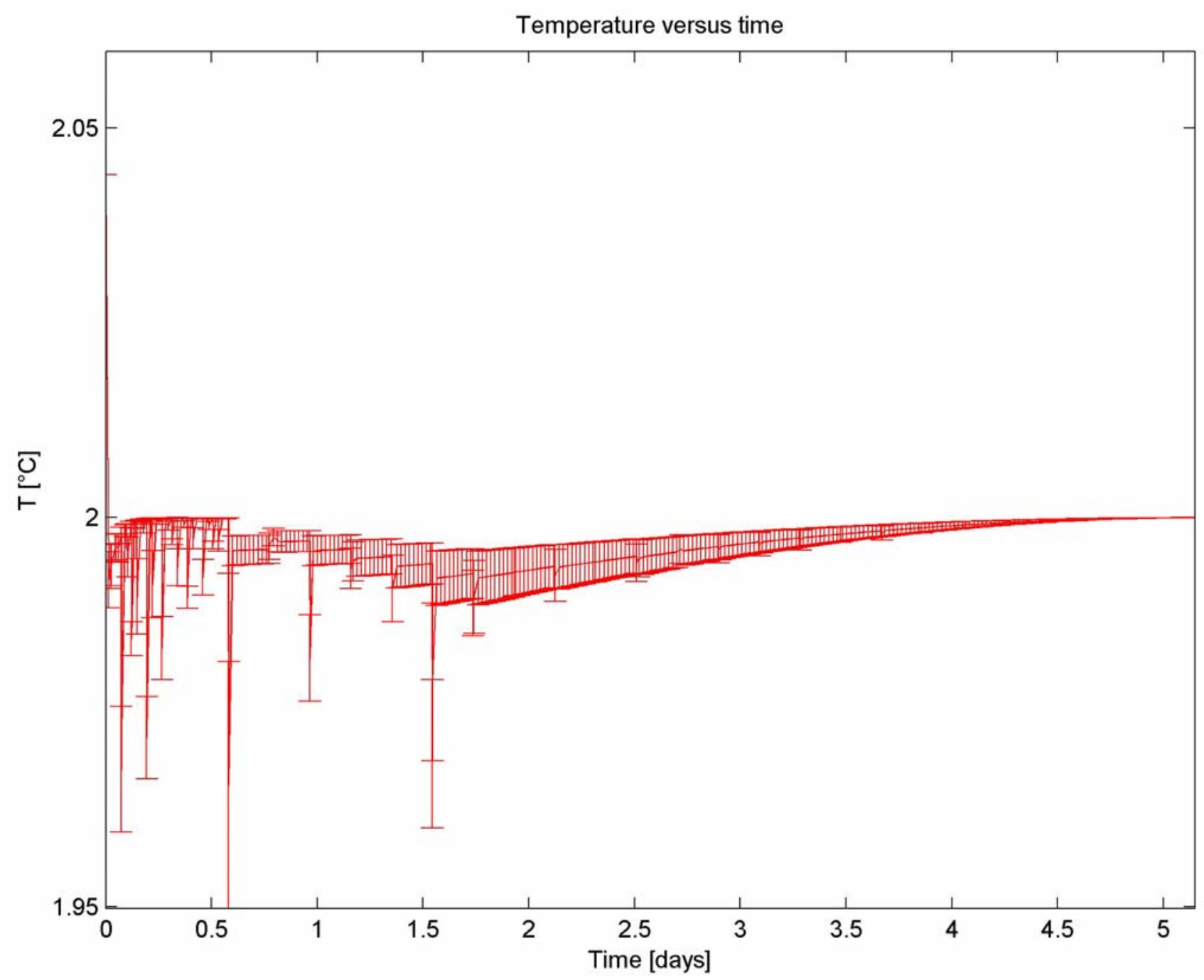


Figure 3.2.31. Test 13: Average Hydrate Saturation throughout the Core with Time The Error Bar is the Variation in all the Grid Blocks

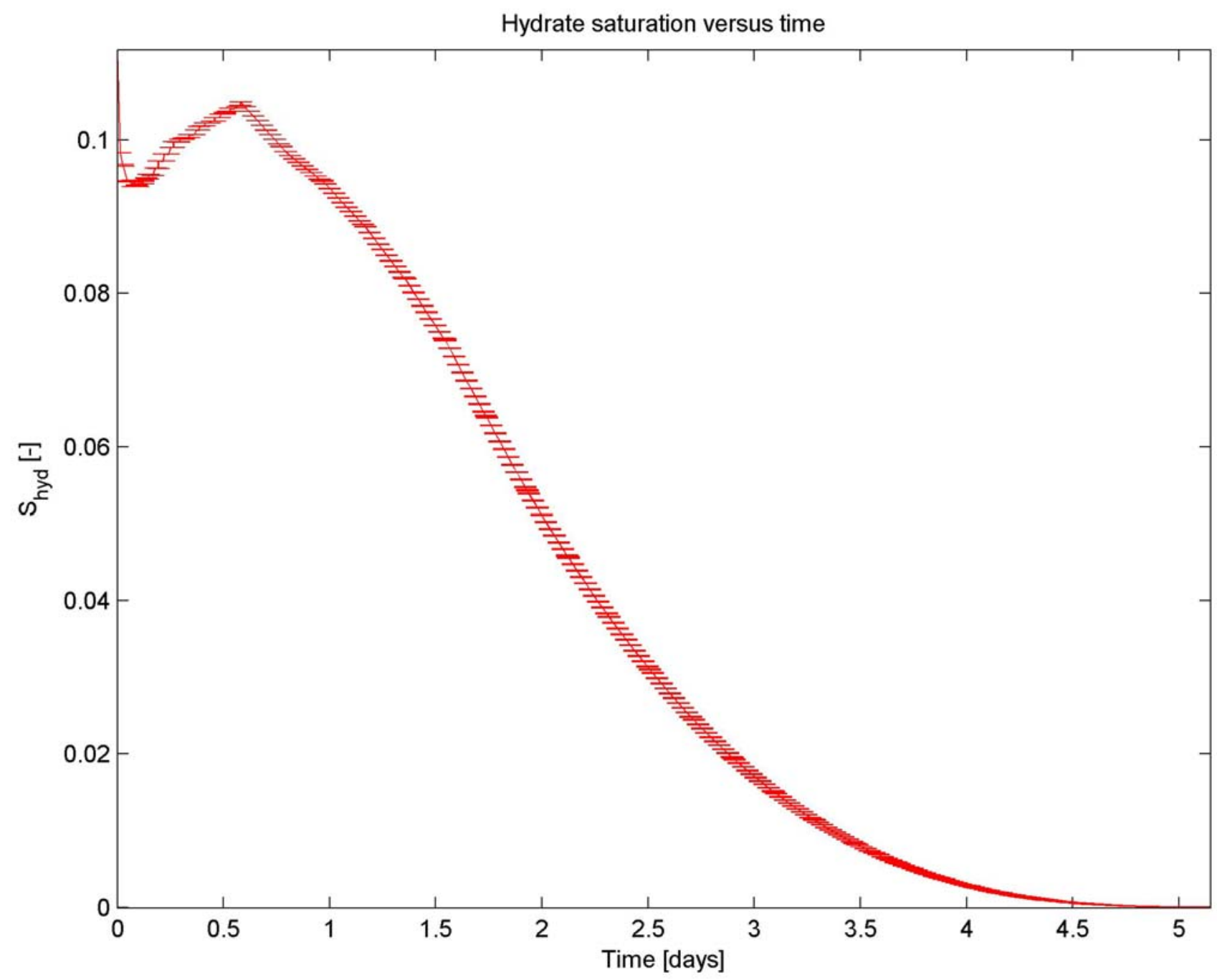


Figure 3.2.32. Test 13: Average Aqueous Saturation throughout the Core with Time The Error Bar is the Variation in all the Grid Blocks

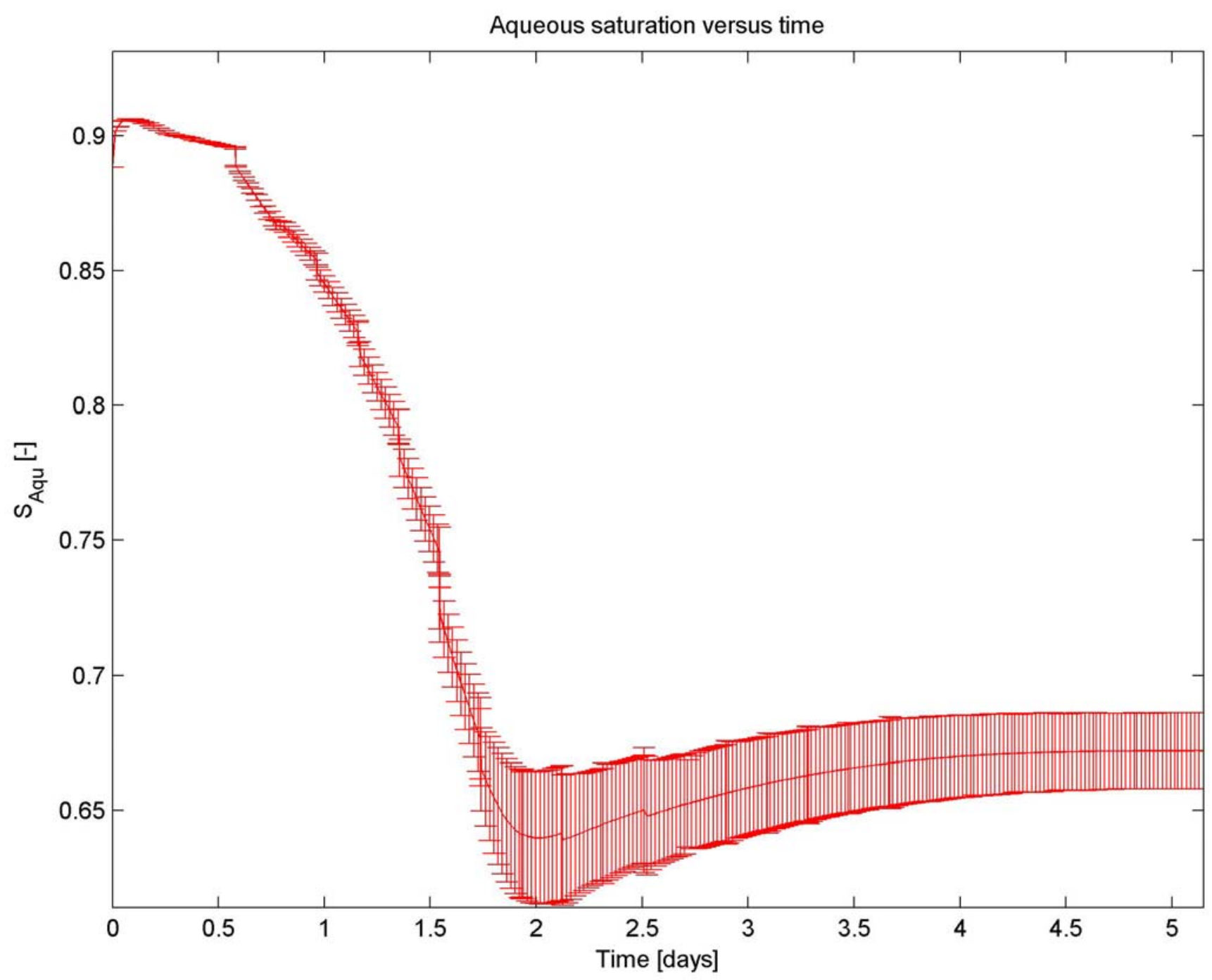


Figure 3.2.33. Test 13: Average Gas Saturation throughout the Core with Time The Error Bar is the Variation in all the Grid Blocks

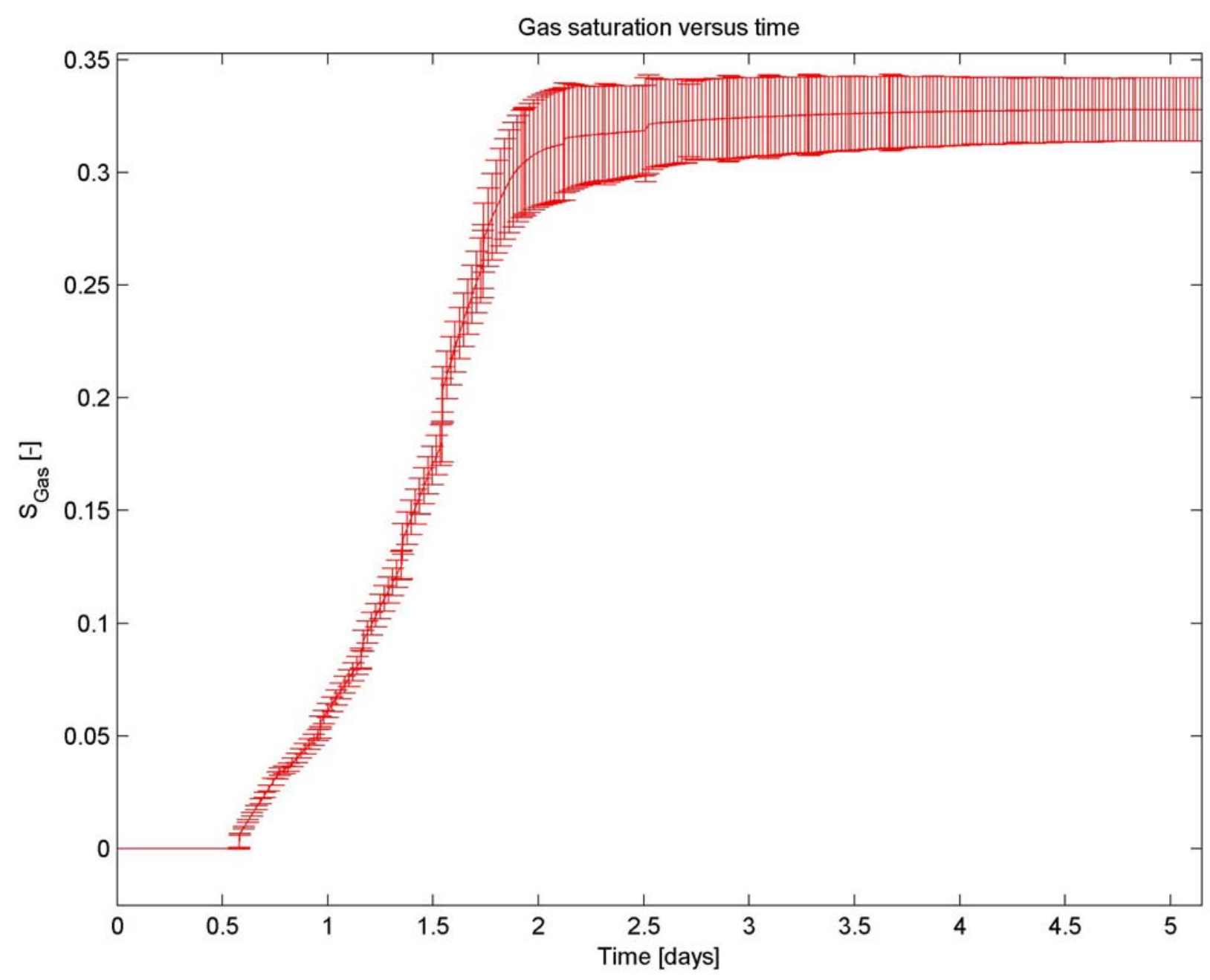


Figure 3.2.34. Test 13: Total Volume of Gas, Hydrate, Aqueous and Ice in the Core with Time The Error Bar is the Variation in all the Grid Blocks

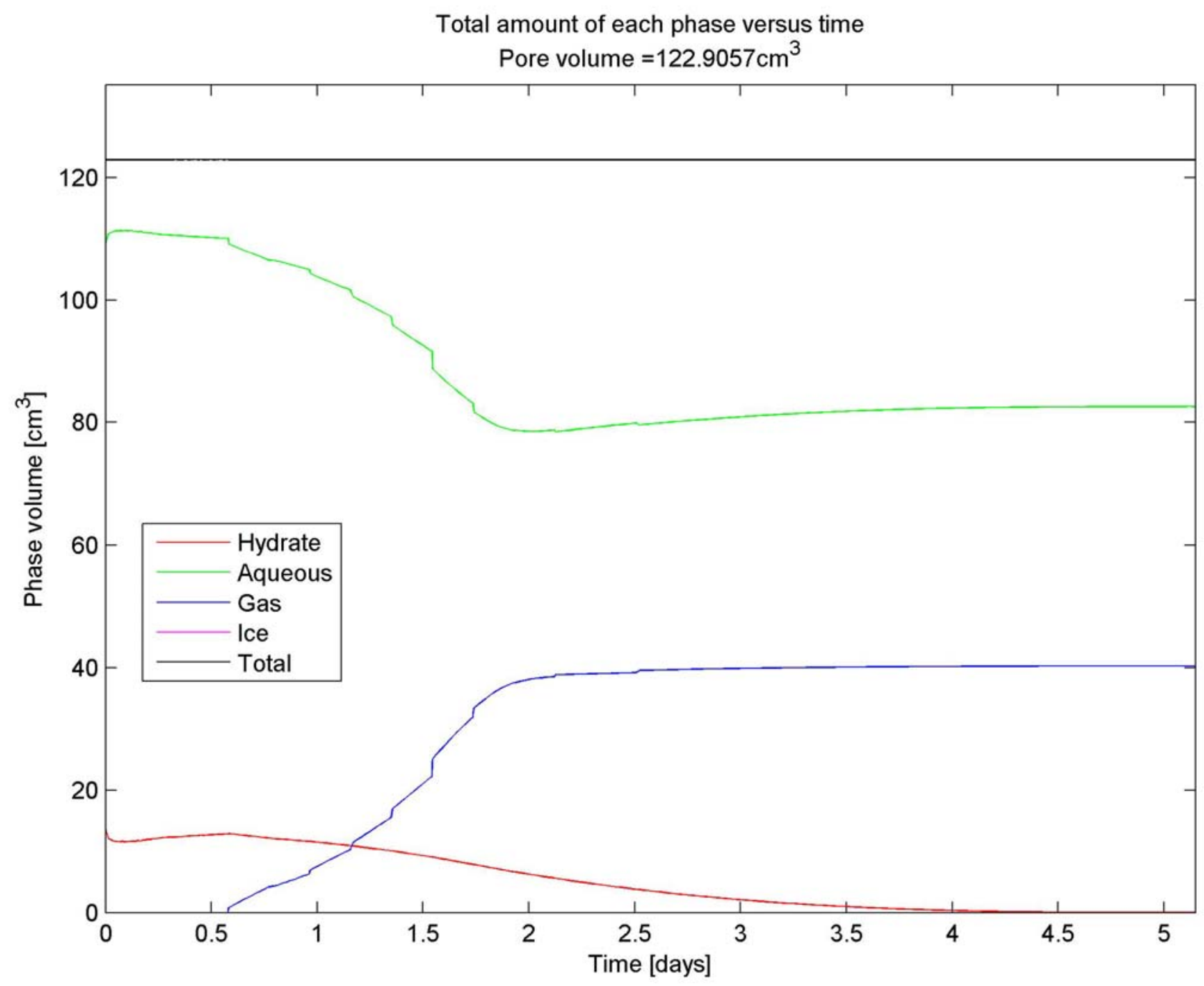




\section{Figure 3.2.35. Test 13: Cumulative Volume of Methane Released over a Period of Time}

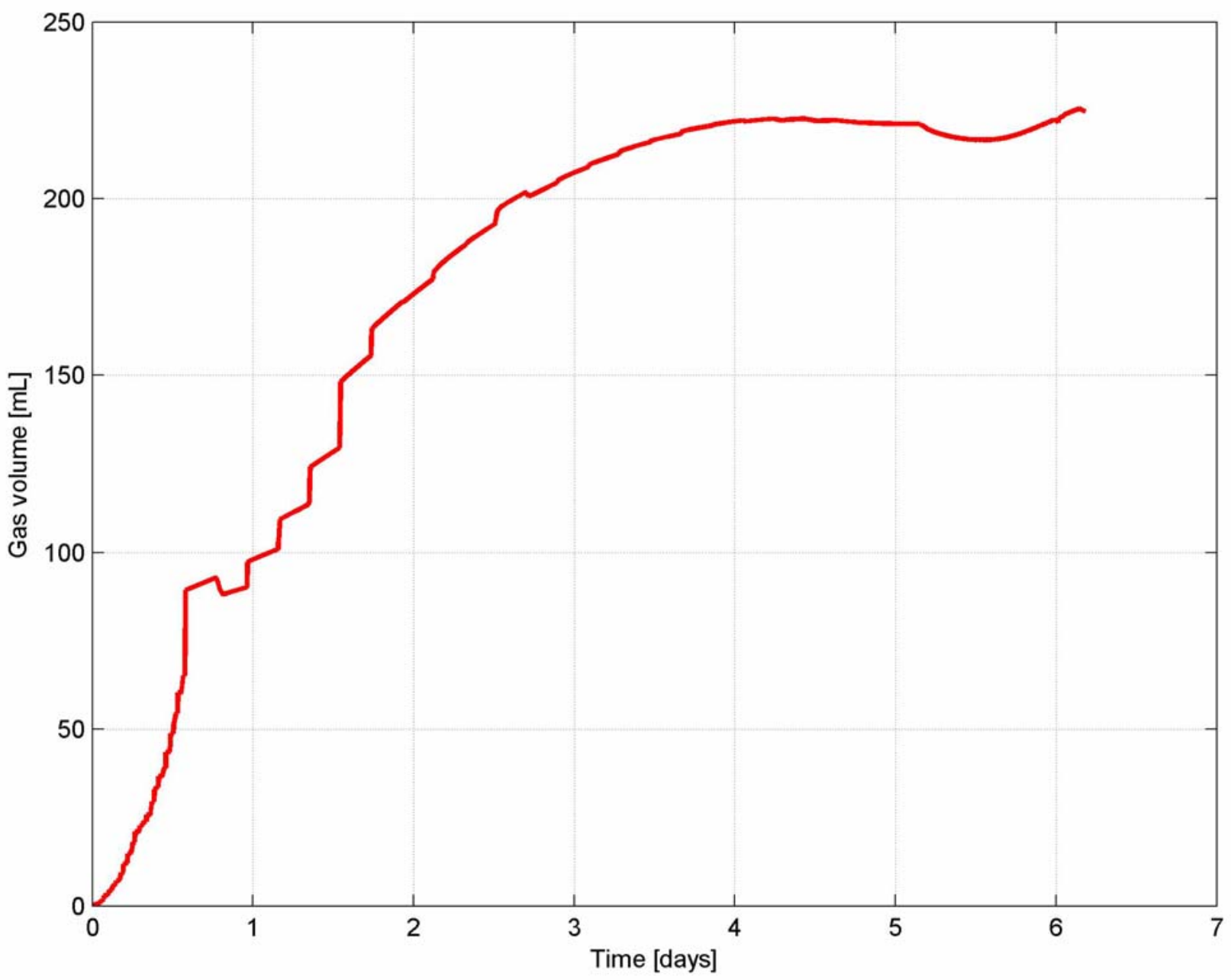




\subsection{Technology Transfer}

As a result of the original modeling effort, a one-dimensional reservoir model was produced. This model was the basis for the thesis authored by Dr. Xuefei Sun for the University of Houston in 2003, "Gas Production from Methane Hydrate Sediments: A One-Dimensional Model" Dr. Sun and Professor Kishore Mohanty of the University of Houston published the following papers:

- $\quad$ "1-D Modeling of Hydrate Depressurization in Porous Media," Transport in Porous Media, Vol. 58, 315-338 (2005)

- "Kinetic Simulation of Methane Hydrate Formation and Dissociation in Porous Media," Chemical Engineering Science, Vol. 61, 3476-3495 (2006)

Preliminary results of the experimental work were included in a presentation by Dr. Sun and Professor Mohanty, "Simulation of Methane Hydrate Reservoirs," at the Society of Petroleum Engineers Reservoir Simulation Symposium conducted at The Woodlands, Texas, January 31 through February 2, 2005. 


\subsection{CONCLUSIONS}

- A novel core holder was developed that allowed application of net confining stress, CT scanning, measurement of electrical resistivity, acoustic velocity, and in situ temperature during hydrate formation and dissociation (Figure 1.2).

- During hydrate formation in porous materials acoustic velocity drops (Figure 3.1.5), electrical resistivity increases (Figure 3.1.13), x-ray absorption (CT numbers) decreases in brine-saturated regions and increases in gas-saturated regions (Figure 3.1.9). These measurements can be used to detect the in situ hydrate distribution.

- Core tests of hydrate formation can be conducted successfully if water is injected to maintain the high pressure during hydrate formation (Tests 10-13). When methane gas was injected to maintain the high pressure during the hydrate formation, the hydrate formed in the supply side of the core and plugged the core hydraulically disconnecting the other side of the core (Tests 3-9).

- Depressurization of hydrates within cores can be conducted successfully at a constant fluid withdrawal rate. The production of water and gas was monitored by an online densitometer (Tests 10-13). Leaks developed in several experiments with hydrate dissociation by temperature increase (Test 6).

- Hydrate depressurization experiments can be matched qualitatively by the hydrate simulator (simulations of Tests 10-13). A series of simulations were run to mimic the variable pressure conditions at the production well. The simulator was able to apply constant pressure boundary condition, but not the constant total volumetric flow rate boundary condition.

- The core temperature falls temporarily during hydrate dissociation (and increases temporarily during hydrate formation). The temperature drop is higher if the fluid withdrawal rate is higher (Tests 10-13). This is due to the heat of hydrate formation. This heat is supplied to the dissociation front from the sensible heat, which lowers the temperature of the porous medium in the vicinity.

- The sodium iodide concentration affects dissociation pressure and rate. (Figure 1.8 shows that the effect could be of the order of 600 psi.) 
- The pressure drops (flow effects) are small within the core (3.1.12). Very small pressure gradients were observed in most of the experiments except when the cores became plugged. The flow effects are expected to be larger in the field scale and may control hydrate dissociation.

- At the core scale, hydrate depressurization is kinetically controlled (Figure 3.2.23), because pressure drops are small. Changes in the kinetic rate constant affect the rate of methane production. At the field scale, hydrate depressurization may be controlled by other factors such as flow effects and heat transfer. 


\section{ACKNOWLEDGEMENTS}

We wish to acknowledge the participation of the following who contributed to the success of this project:

National Energy Technology Laboratory, US Department of Energy

Dr. John Rogers

Tom Mroz

Gary Sames

Richard Baker

\section{Advisory Board}

Dr. John Rogers, National Energy Technology Laboratory

Dr. Emrys Jones, ChevronTexaco JIP

Prof. Turgay Ertekin, Pennsylvania State University

Dr. Vik Rao, Technology Ventures, Halliburton Energy Services Inc.

Dr. Richard Drozd, Westport Technology Center

Principal Investigators

Dr. Bayram Kalpakci

Dr. Deepak Gupta

Project Coordinator

John P. Shillinglaw

Westport Technical Staff

Thomas Ballweg

Rogelio Banda

Scott Brown

Dr. Jon Burger

William Cook

Doug Frank

Mustafa Hakimuddin

Pat Jacobs

Dr. Aftab Khokhar

Daniel Mata

Lee Nuebling

Mickey Pelletier

Jeremy Rohan

John Rohan

Dr. Keshawa Shukla

John Welch

\section{University of Houston}

Professor Kishore Mohanty

Ogunlana, Damilola, Post-doctorate, Chemical Engineering

Pitchumani, Ramanan, Post-doctorate in progress, Chemical Engineering

Nanchary, Narender, M.S., 2003, Chemical Engineering

Sun, Xuefei, Ph.D., 2004, Chemical Engineering

\section{National Institute of Advanced Industrial Science and Technology}

Dr. Hideki Minagawa

Dr. Hide Narita 


\section{REFERENCES}

Clarke, M. and Bishnoi, P. R. (2000), Determination of the intrinsic rate of ethane gas hydrate decomposition, Chem. Eng. Sci. 55, 4869-4883.

Clarke, M. and Bishnoi, P. R. (2001), Determination of the active energy and intrinsic rate constant of methane gas hydrate decomposition, Can. J. of Chem. Eng., 79 (2), 143-147.

Ji, C., Ahmadi, G., and Smith, D. H. (2001), Natural gas production from hydrate decomposition by depressurization, Chem. Eng. Sci. 56, 5801-5814.

Hippe, Fabian W., Humphrey, Gary D. and Tjok, Ko-Min (2006), Results of core sample analysis, standard and advanced laboratory testing, Chevron GOM Gas Hydrates JIP, Report 0201-5081, 177-178.

Kim, H.C., Bishnoi, P. R., Heidemann, R. A. and Rizvi, S. S. H. (1987), Kinetics of methane hydrate dissociation, Chem. Eng. Sci. 42 (7), 1645-1653.

Kvenvolden, K. A., Carlson, P. R. and Threlkeld, C. N. (1993), Possible connection between two Alaskan catastrophes occurring $25 \mathrm{yr}$ apart (1964 and 1989), Geology, 21, 813-816.

Makogan, Y. F. (1997), Hydrates of hydrocarbons, PennWell Books, Tulsa, Oklahoma.

Makogon, Y. F. and Holditch, S. A. (2001), Experiments illustrate hydrate morphology and kinetics, Gas and Oil J., 45-50.

Moniz, E. J. \& Kenderdine, M. A. (2002), Meeting energy challenges: technology and policy, Physics Today, 55, 40-46.

Moridis, G. J. (2002), Numerical studies of gas production from methane hydrates, SPE 60693, proceedings of the SPE Gas Technology Symposium, Calgary, April 30-May 2.

Nozad, I., Carbonell, R. G. and Whitaker, S. (1985), Heat conduction in multiphase systems. I. Theory and experiment for two-phase systems, Chem. Eng. Sci. 40, 843-855.

Selim, M. S. and Sloan, E. D. (1989), Heat and mass transfer during the dissociation of hydrate in porous media, AlChE J., 35, 1049-1052.

Silva, A. J,, Brandes H. G., and Veyera G. E., (1996), Geotechnical Characterization of Surficial HighPorosity Sediments in Eckernforde Bay, Geo-Marine Letters, 16, 167-184.

Skov, A. M. (2003), World energy beyond 2050, J. of Petroleum Tech., 55 (1), 34-37.

Sloan, E. D. (1998), Clathrate Hydrates of Natural gases, $2^{\text {nd }}$ ed., Marcel Dekker, New York.

Stern, L. A., Kirby, S. H., and Durham, W. B. (1996), Peculiarities of methane clathrate hydrate formation and solid-state deformation, including possible superheating of water ice, Science, 273, 1843-1848.

Tester, J. W. (1997), Thermodynamics and Its Applications, $3^{\text {rd }}$ ed., Prentice Hall, New Jersey.

Tsypkin, G. G. (1991), Effect of liquid phase mobility on gas hydrate dissociation in reservoirs, J. of Fluid and Gas Mechanics, 4, 105-114.

Wilder, J. W. and Smith, D. H. (2002), Dependencies of clathrate hydrate dissociation fugacities on the inverse temperature and inverse pore radius, Ind. Eng. Chem. Res. 41, 2819-2825.

Yousif, M. H., Abass, H. H., Selim, M. S. and Sloan, E. D. (1991), Experimental and theoretical investigation of methane-gas-hydrate dissociation in porous media, SPE Res. Eng. 6, 69-76.

Moridis, G. J., Collett, T. S., Dallimore, S. R., Satoh, T., Hancock, S. and Weatherill, B. (2002), "Numerical studies of gas production from several $\mathrm{CH}$ 4-hydrate zones at the Mallik site, Mackenzie Delta, Canada," LBNL-50257, Lawrence Berkley National Laboratory, Berkley, CA. 
Nimblett, J. and Ruppel, C. (2003), Permeability evolution during the formation of gas hydrates in marine sediments, Journal of Geophysical Research, 108 (B9), 2420.

Sun, X. \& Mohanty, K. K. (2006), Kinetic Simulation of Methane Hydrate Formation and Dissociation in Porous Media, Chem. Eng. Sci., 61, 3476-3495.

Sun, X. \& Mohanty, K. K. (2005), Simulation of Methane Hydrate Reservoirs, SPE 93015, Proceedings of SPE Reservoir Simulation Symposium, Woodlands, TX, Jan. 31-Feb. 2, 2005.

Waite, W. F.; de Martin, B. J.; Kirby, S. H.; Pinkston, J.; Ruppel, C. D. (2002) Thermal Conductivity Measurements in Porous Mixtures of Methane Hydrate and Quartz Sand, Geophys. Res. Lett., 29 (24), 2229.

Yun, T.-S., F. Francisca, J.C. Santamarina, and C. Ruppel (2005), Compressional and shear wave velocities of uncemented sediment containing gas hydrate, Geophys. Res. Lett., 32, L10609.

Moridis, G.J., Kowalsky, M.B., and Pruess, K. (2005), Depressurization-Induced Gas Production From Class 1 Hydrate Deposits, SPE 97266, SPE ATCE, Dallas, TX, 9-12 October, 2005. 


\section{APPENDIX A \\ Thin Section Descriptions}


DOE

Gas Hydrates Research

Synthetic Porous Media

$\begin{array}{ll}\text { Sample Number: } & \text { QF-50 } \\ \text { Depth (ft): } & \\ \text { Porosity, percent: } & \mathbf{1 6 . 4} \\ \text { Gas Permeability, md: } & \mathbf{0 . 0 1 8} \\ \text { Grain Density }\left(\mathrm{g} / \mathrm{cm}^{3}\right) \text { : } & \mathbf{2 . 6 7 0} \\ \text { Sample Type: } & \text { Plug }\end{array}$

\section{Thin Section Description:}

The photomicrographs on the facing page are thin section and image processed views of the synthetic quartz based 50 Darcy sandstone sample. This sample consists of loose quartz sand grains that have been indurated by epoxy resin.

\section{Plate 1A -}

Low magnification thin section photomicrograph of the quartz based 50 Darcy sandstone sample. The rock consists of subround, loosely packed quartz grains (white, J-14). Intergranular porosity is well developed (blue). Pores are quite large and very well interconnected through very large pore throat openings. The rock is much coarser grained and much more loosely packed than either the Berea Sandstone or the quartz based 10 Darcy sandstone sample.

\section{Plate 1B -}

High magnification view illustrating development of intergranular porosity (blue). The rock consists of subround to round, loosely packed quartz sand grains (white, $\mathrm{H}-6$ ) that are indurated by meniscus of epoxy resin (white, $\mathrm{H}-13, \mathrm{~L}-12$ ). Due to relatively limited compaction and epoxy resin impregnation, the sample has very high porosity and permeability.

Plate 1C Image processed binary view of photo A. This photograph illustrates pore space distribution. Porosity appears black in this photomicrograph.

\section{Plate 1D -}

False-color image processed view of photos $A$ and $C$ illustrating variations in pore size and pore size distribution within the sample. The following graph displays the color scale. 
Sample: QF-50

\section{Plate $1 A$}

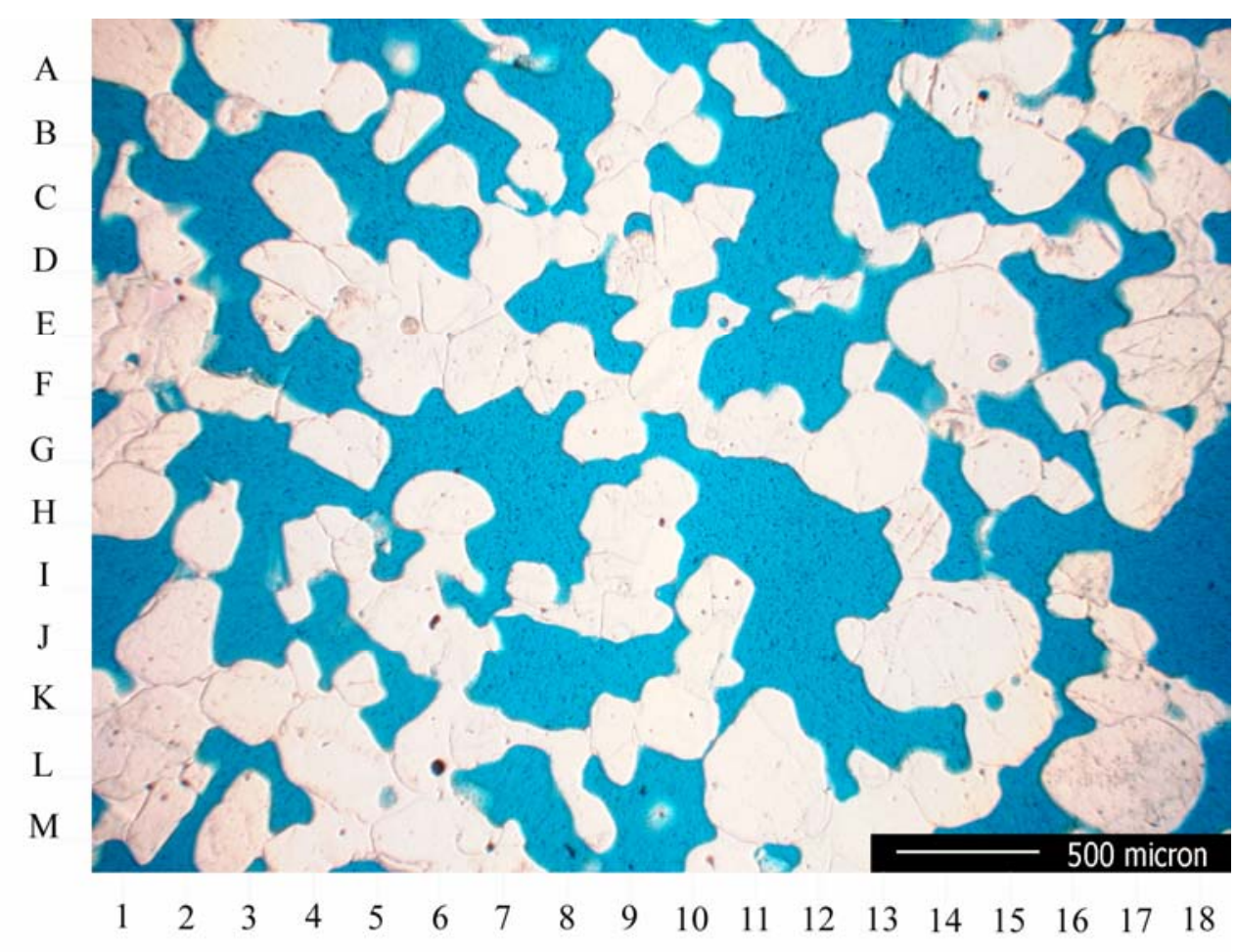

Plate $1 B$ 


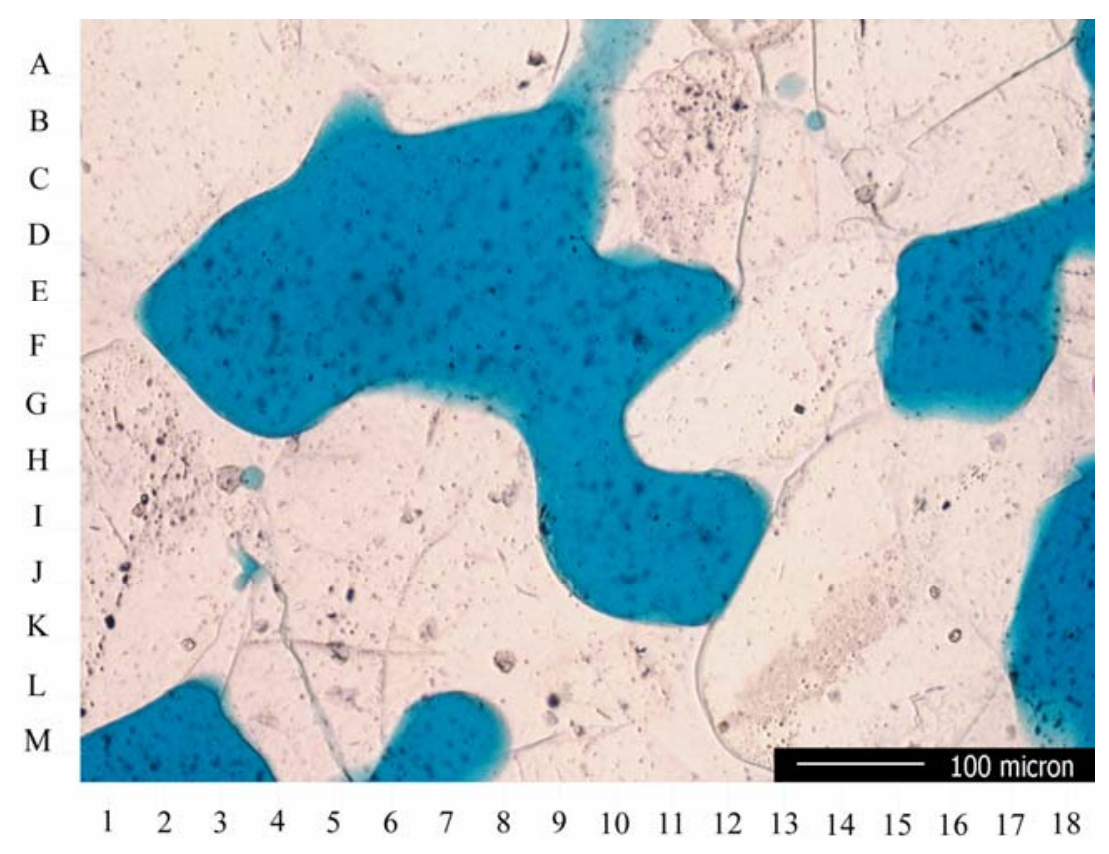

Sample: QF-50

Plate $1 C$

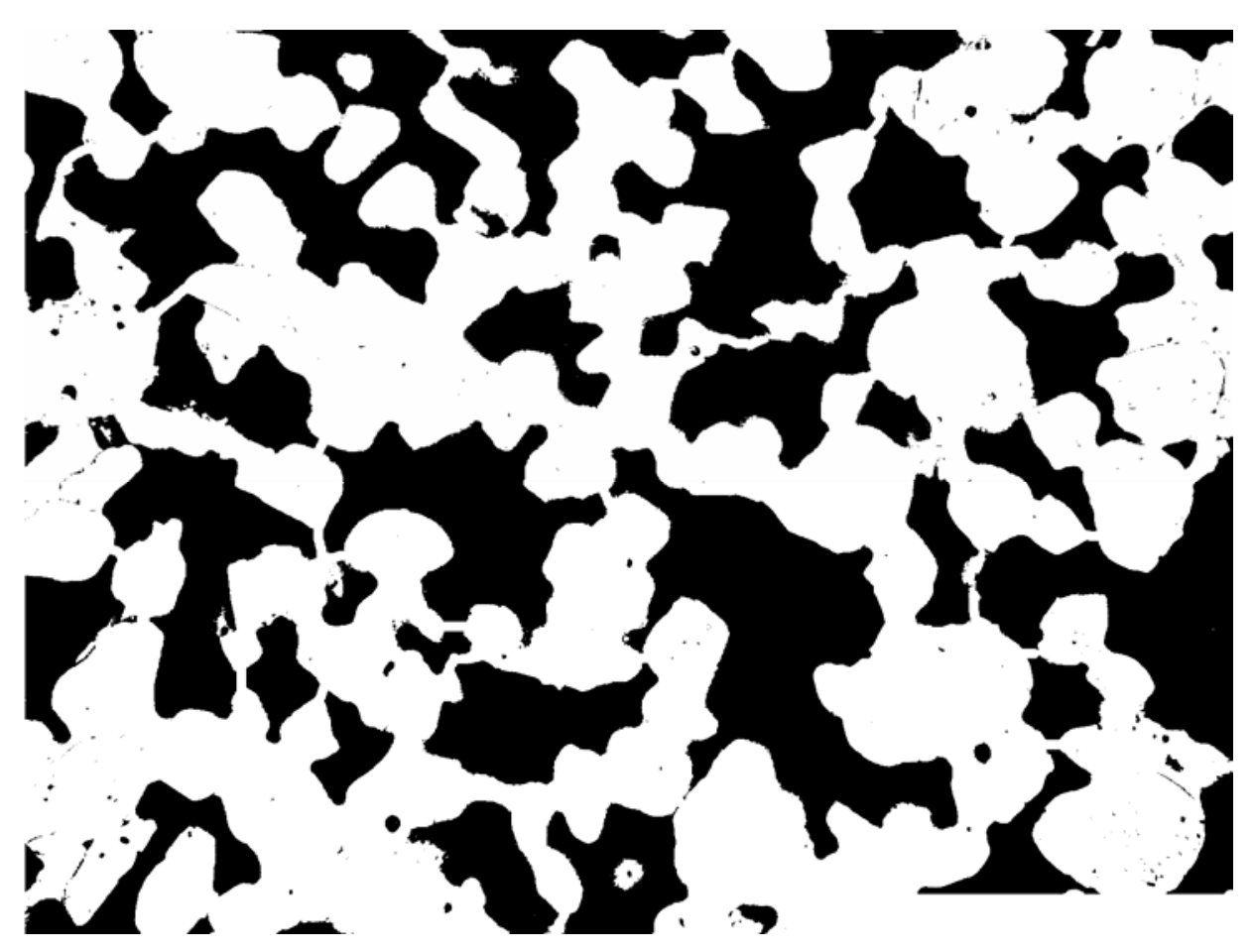

Plate 1D 


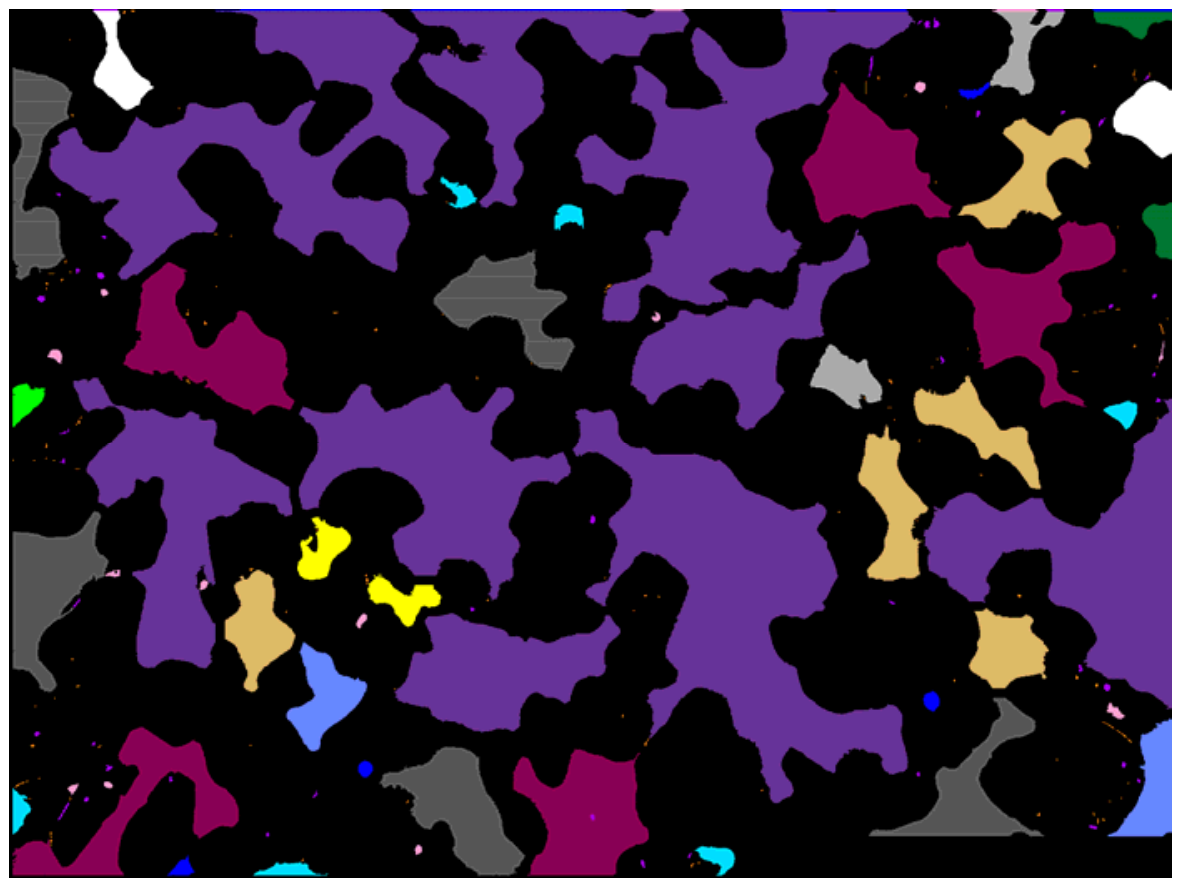

DOE

Gas Hydrates Research

Synthetic Porous Media

Sample Number:

QF-50

Depth (ft):

Porosity, percent:

Gas Permeability, md:

Grain Density $\left(\mathrm{g} / \mathrm{cm}^{3}\right)$ :

Sample Type:

Plug

\section{Scanning Electron Photomicrographs Description:}

\section{Plate 2A -}

Scanning electron photomicrograph of the interior of an intergranular pore within the 50 Darcy synthetic sandstone sample. The rock consists of subround to round, loosely packed quartz grains (F-5, F-12) bound by meniscus of epoxy resin (J-14). Intergranular porosity (H-9) is well developed. Pores are quite large (in excess of $200 \mu \mathrm{m}$ ) and well interconnected, factors contributing to very high permeability.

\section{Plate 2B -}

High magnification view illustrating well developed intergranular porosity (G-10). The 
pore is in excess of $250 \mu \mathrm{m}$ in diameter and interconnected through pore throat openings several microns in size. Grains (K-6) are partially indurated by epoxy resin (D-17).

\section{Sample: QF-50}

\section{Plate $2 A$}

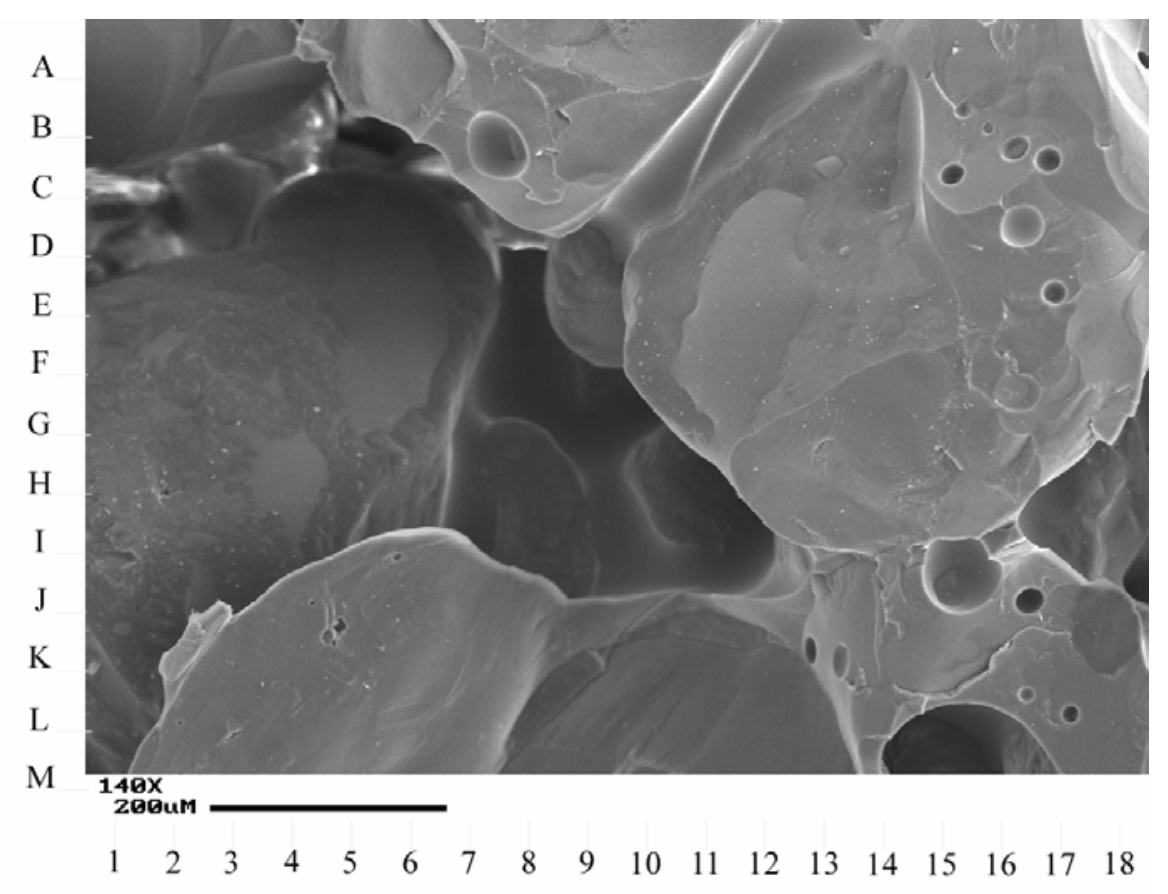

Plate 2B 


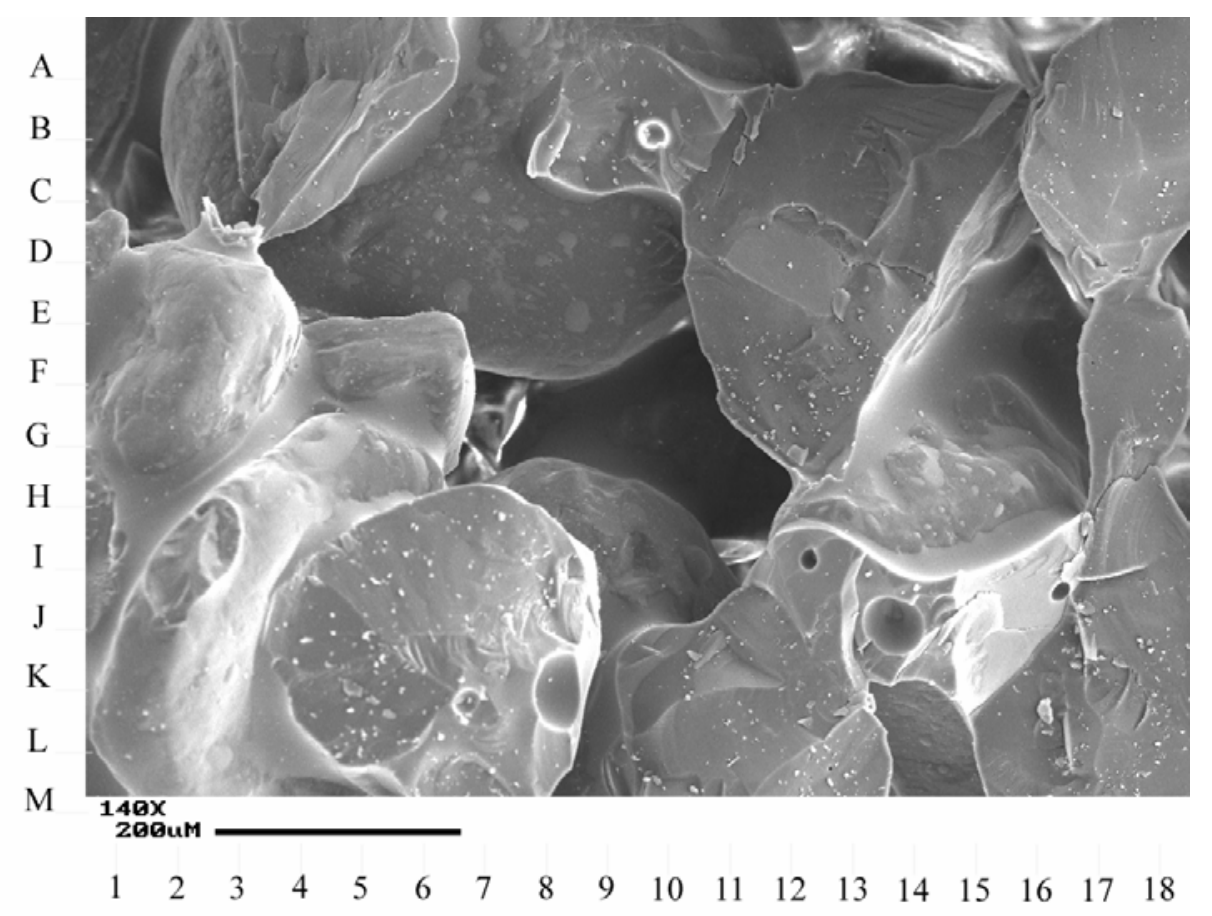

DOE

Gas Hydrates Research

Synthetic Porous Media

Sample Number: $\quad$ QF-10

Depth (ft):

Porosity, percent:

Gas Permeability, md:

Grain Density $\left(\mathrm{g} / \mathrm{cm}^{3}\right)$ :

Sample Type:

Plug

\section{Thin Section Description:}

Photomicrographs on the facing page display thin section and image processed views of the synthetic quartz based 10 Darcy sandstone sample.

\section{Plate 3A -}

Low magnification thin section photomicrograph illustrating the fine grained $(0.21 \mathrm{~mm})$, well sorted nature of rocks comprising this portion of the sequence. Framework grains consist predominantly of monocrystalline quartz (white, G-7). The rock contains small amounts of fractured grain residue (greenish residue in scattered pore space, I-8). Note 
the close packing of framework sand grains resulting from compaction presumably during sample preparation. Grains are indurated by epoxy resin. The rock is devoid of natural cements. Note the rather patchy distribution of open and closer packing within the sample. These packing heterogeneities are partially responsible for pore size and permeability variations within the sample. The rock is characterized by a very large standard deviation of pore sizes. All of the porosity is intergranular in nature (blue).

\section{Plate 3B -}

High magnification view illustrating the development of intergranular porosity (blue), as well as some smaller mesopores associated with fractured grain residue (greenish areas, $\mathrm{C}-12$ ). Intergranular pore space is partially filled by epoxy resin (white meniscus on grains, E-6).

\section{Plate 3C -}

Image processed binary view of photo A illustrating the distribution of porosity. Porosity appears black in this photomicrograph.

\section{Plate 3D -}

False-color image processed view of photos $A$ and $C$. The view is colored to illustrate variations in pore diameter. The following graph displays the color scale.

\section{Sample: QF-10}

\section{Plate $3 A$}

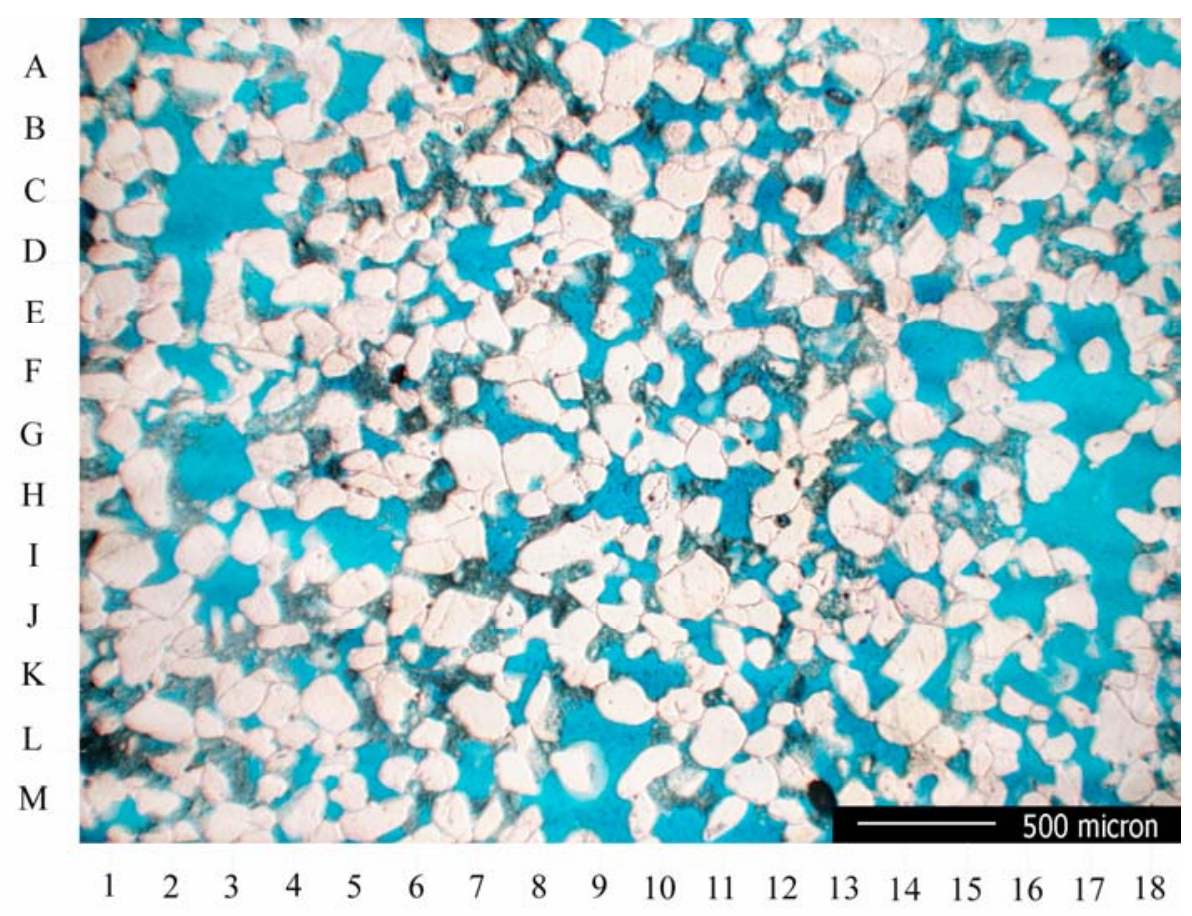

Plate 3B 


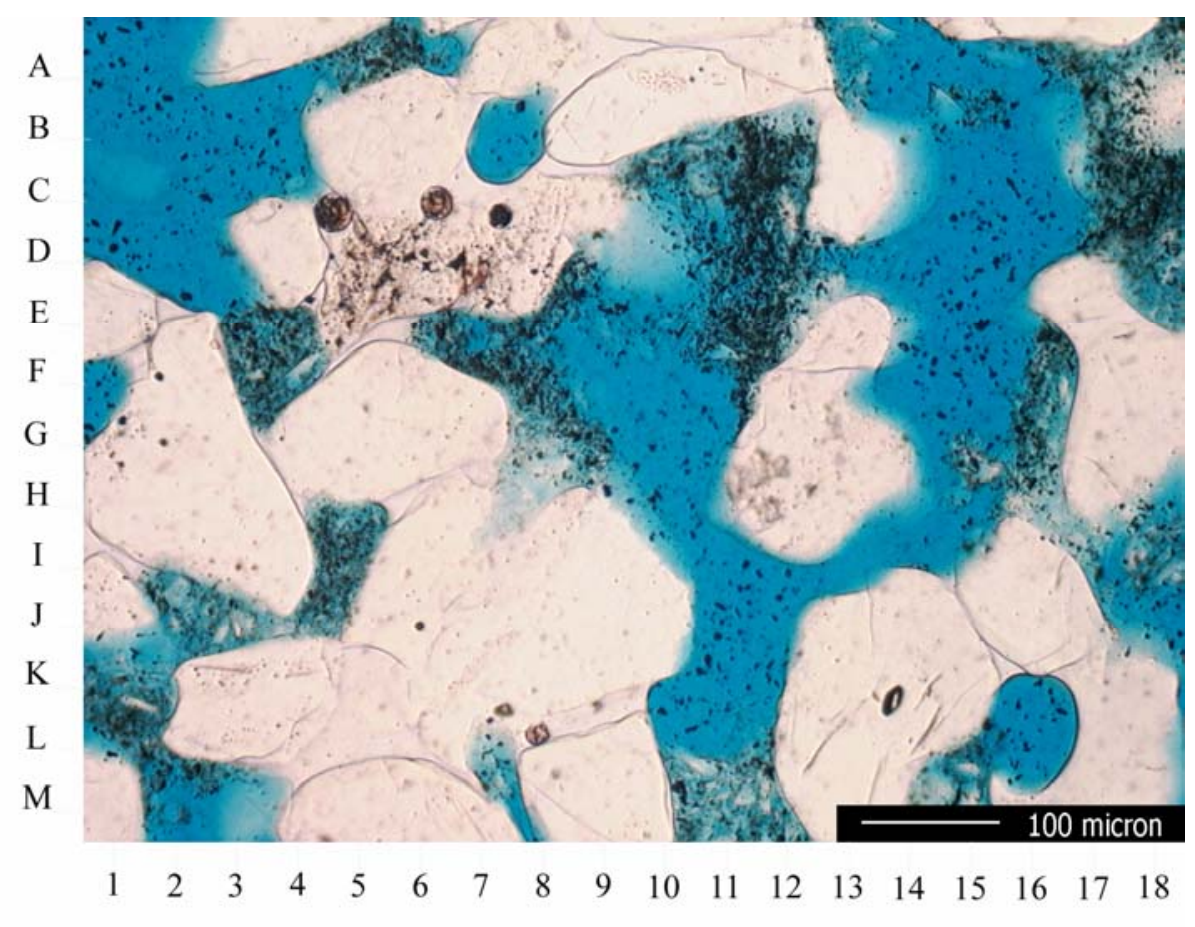

Sample: QF-10

Plate 3C

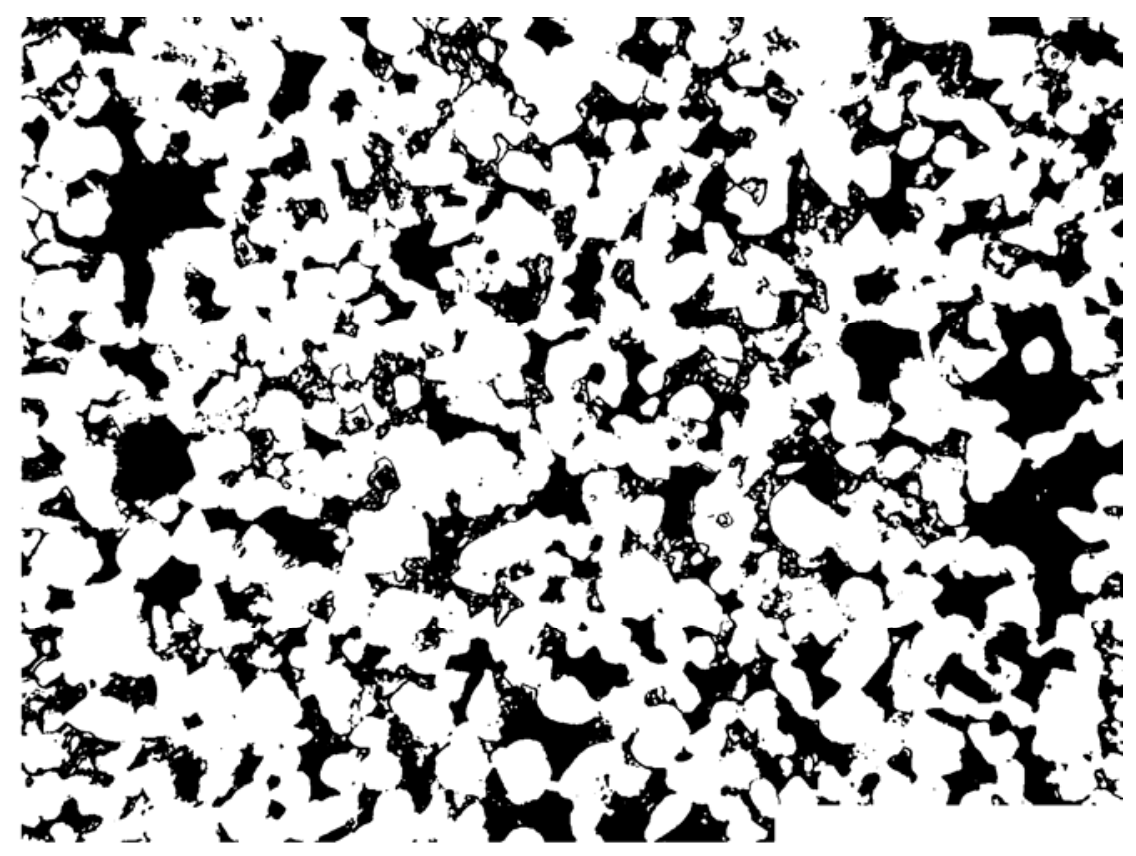

Plate 3D 


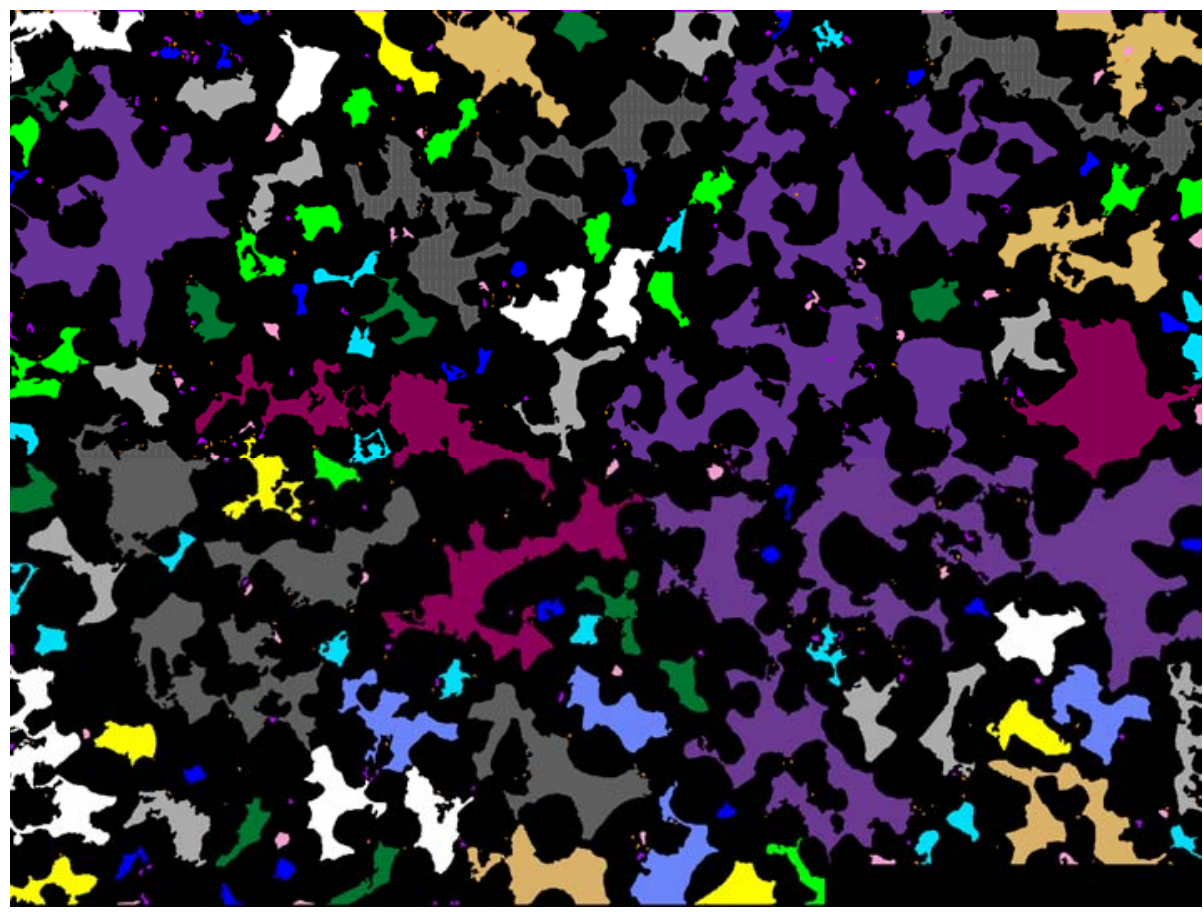

DOE

\section{Gas Hydrates Research} Synthetic Porous Media

Sample Number:

QF-10

Depth (ft):

Porosity, percent:

Gas Permeability, md:

Grain Density $\left(\mathrm{g} / \mathrm{cm}^{3}\right)$ :

Sample Type:

Plug

\section{Scanning Electron Photomicrographs Description:}

\section{Plate 4A -}

Low magnification view illustrating the relatively open packing of subround quartz grains (J-9) from the synthetic quartz based 10 Darcy sandstone sample. Grains have experienced some rotation into a closer packing arrangement in response to compaction applied during sample preparation. The sample is indurated by epoxy resin (E-11). Intergranular porosity is well developed (C-8). Pores range from 50-200 $\mu \mathrm{m}$ in diameter and are well interconnected through pore throats up to several microns in diameter. 


\section{Plate 4B -}

High magnification view of the interior of intergranular pores (D-8). Pores are $<100 \mu \mathrm{m}$ in diameter. Note the impregnation of the sample by epoxy resin (I-9, F-13) providing some consolidation. The rock is devoid of secondary cements.

\section{Plate $4 A$}

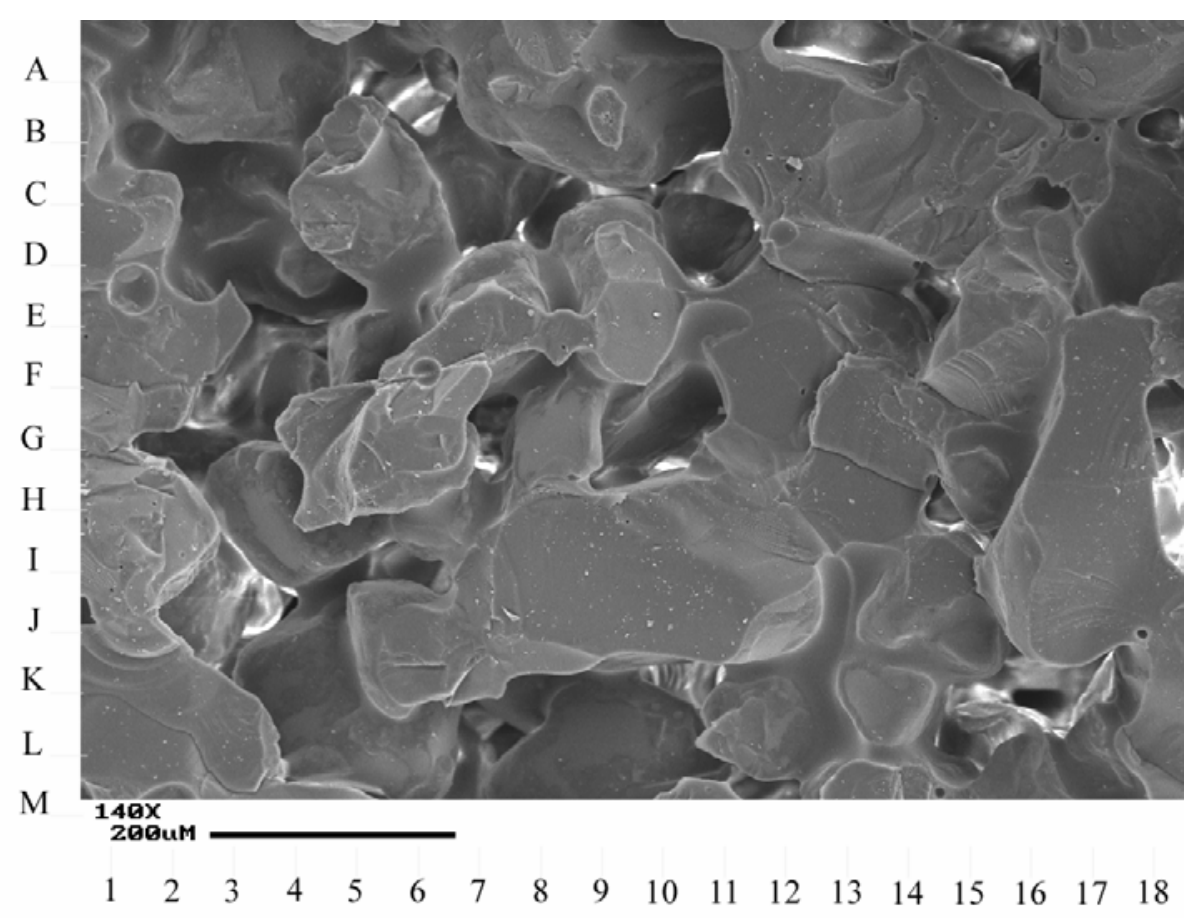

Plate $4 B$ 


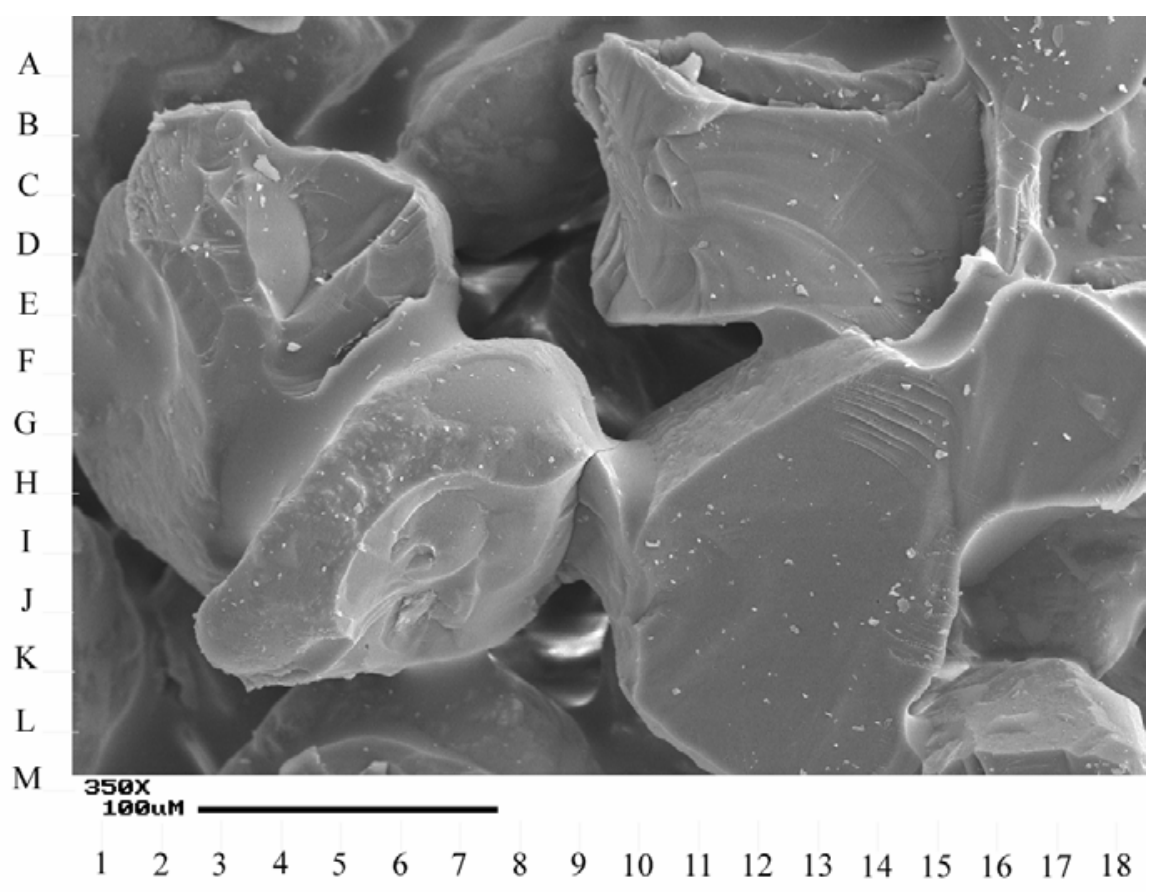

DOE

Gas Hydrates Research Synthetic Porous Media

Sample Number:

Berea Sandstone

Depth (ft):

Porosity, percent:

Gas Permeability, md:

Grain Density $\left(\mathrm{g} / \mathrm{cm}^{3}\right)$ :

Sample Type:

Plug

\section{Thin Section Description:}

Photos A-D on the facing page are color thin section photomicrographs and image processed views of the clean Berea Sandstone.

\section{Plate 5A -}

Low magnification general view illustrating the fine grained $(0.22 \mathrm{~mm})$, well sorted nature of rocks comprising this portion of the sequence. The rock consists predominantly of subangular to subround, close packed grains of monocrystalline quartz (white, G-7), as well as smaller quantities of feldspars and rock fragments (brown). Intergranular pore space is partially filled by dolomite (gold, M-8) and clay cement (milky blue-white areas, F-8). The rock contains small amounts of silica overgrowths, pyrite and siderite cement. The rock is highly porous and moderately permeable. The pore structure is dominated by 
large secondary pores (blue, I-16), large intergranular macropores (blue, E-10) and smaller intergranular pores. Microporosity is associated with clay cements.

\section{Plate 5B -}

High magnification thin section photomicrograph of the clean Berea Sandstone. Note the rotation of grains into a close packing arrangement and development of long grain-tograin contacts (J-16). The rock consists predominantly of monocrystalline quartz grains (G-3), as well as smaller quantities of feldspars (M-12) and rock fragments (D-5). Intergranular pore space (blue) is partially filled by interlocking, euhedral silica overgrowths developed on framework quartz grains (J-16) as well as dolomite. Intergranular pores are partially filled by booklet-like masses of authigenic kaolinite clay cement (milky blue-white areas, E-7).

\section{Plate 5C -}

This view is a processed binary image of photo $A$ and illustrates the distribution of porosity (black) in the sample.

\section{Plate 5D -}

False-color image processed view of photos $A$ and $C$. The view is colored to illustrate variations in pore diameter. The following graph displays the color scale.

\section{Sample: Berea Sandstone}

\section{Plate $5 A$}

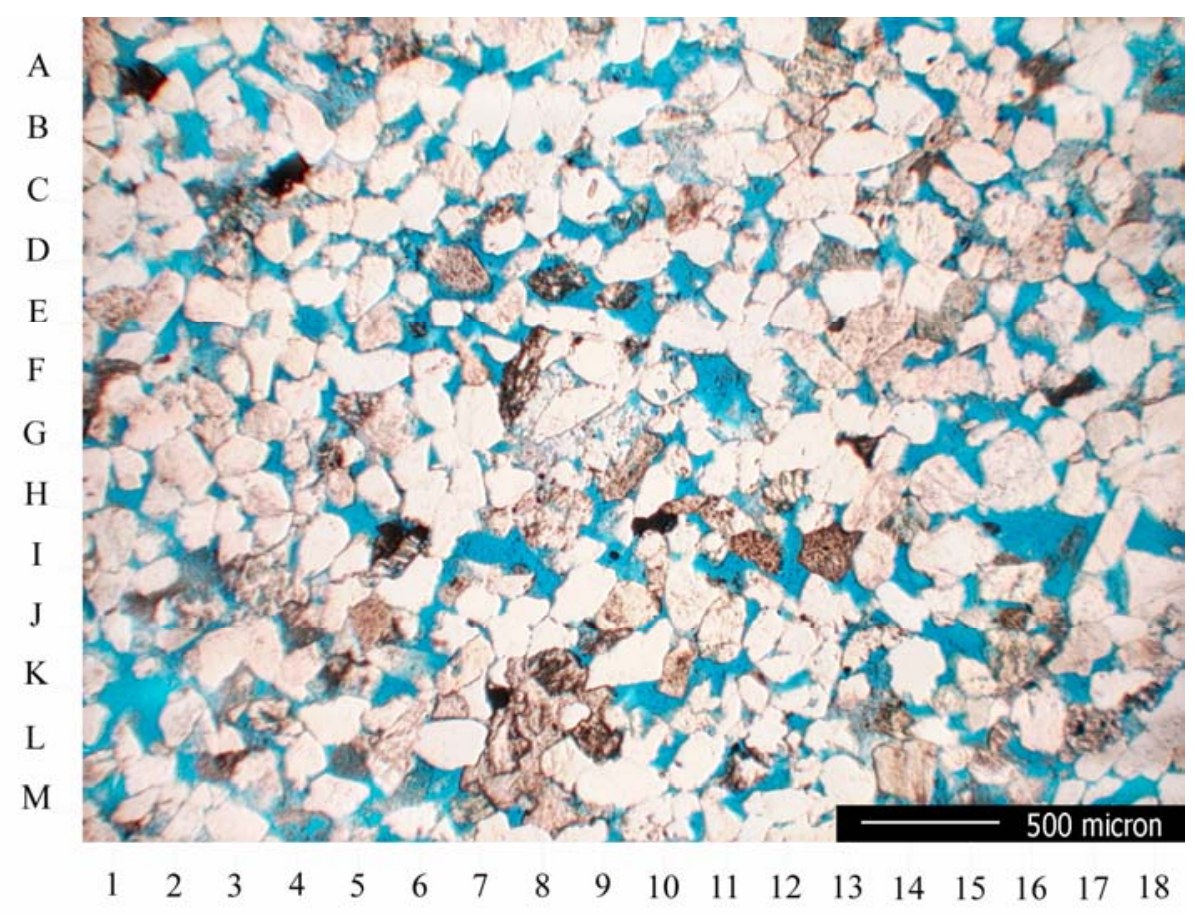

Plate 5B 


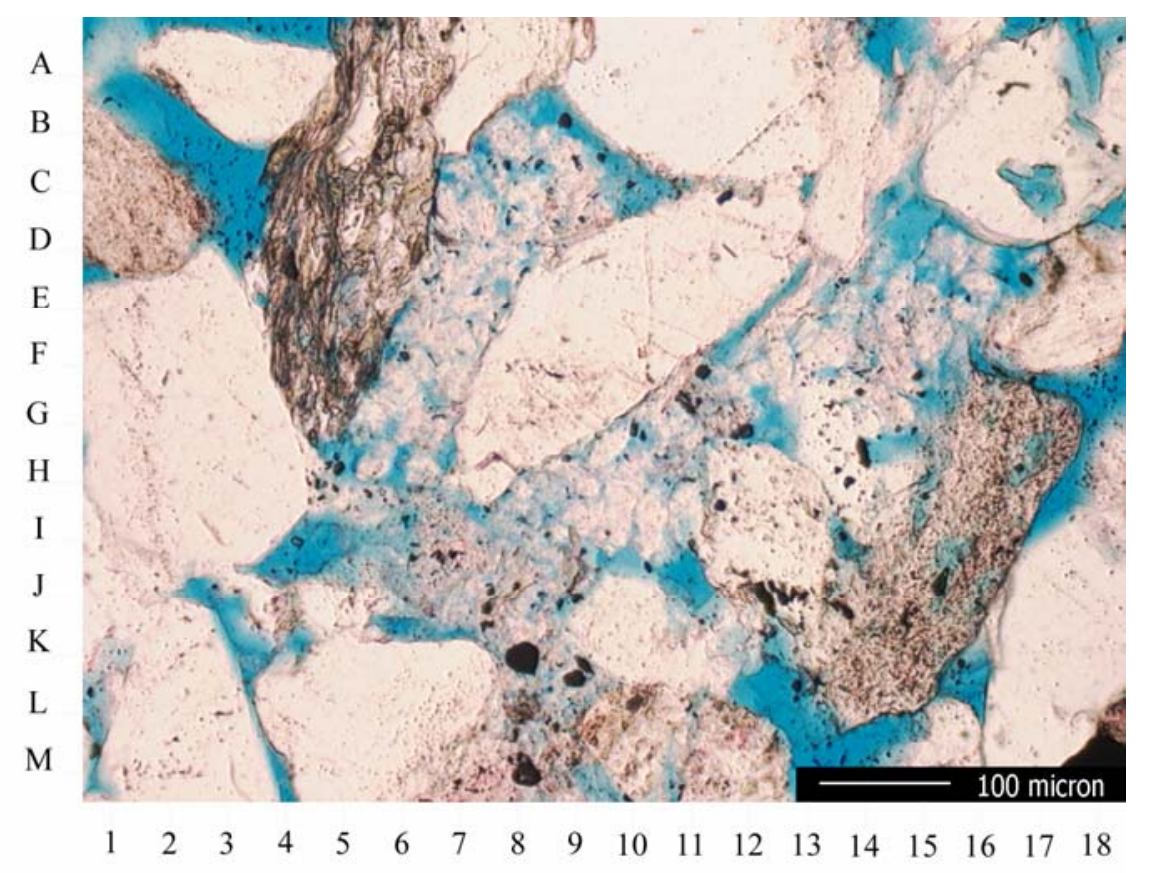

\section{Sample: Berea Sandstone}

\section{Plate 5C}

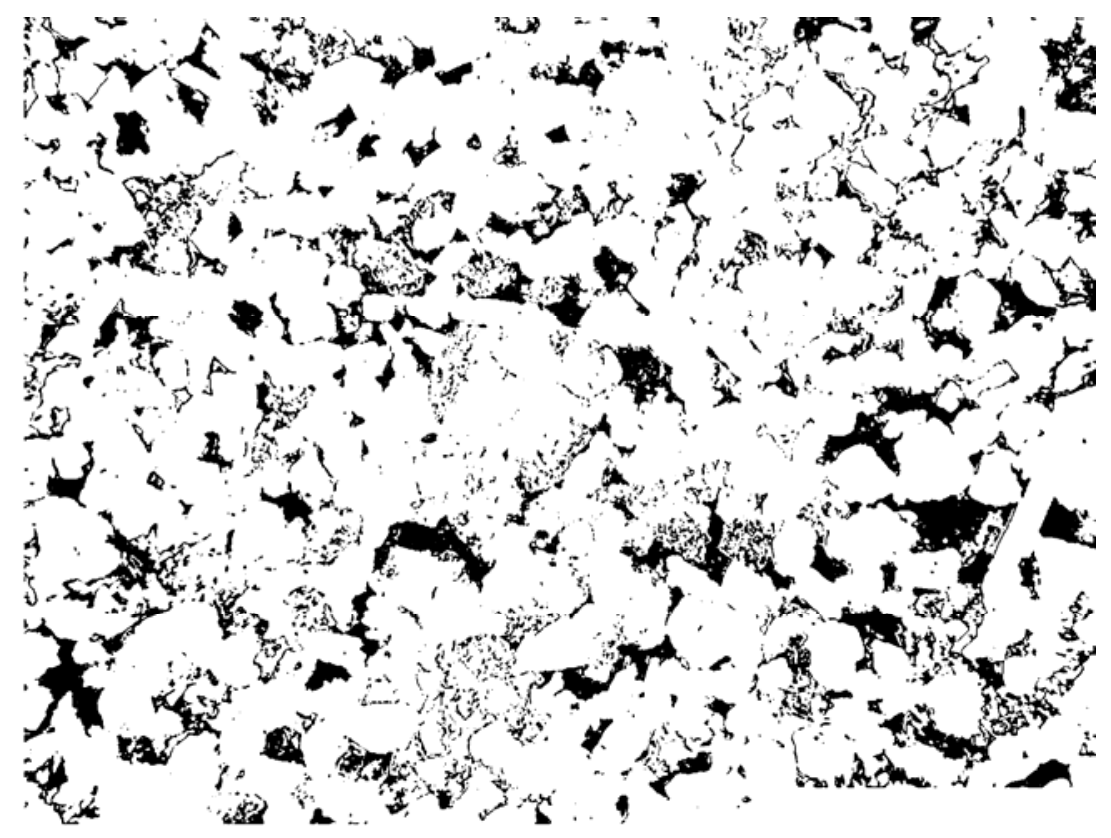

Plate 5D 


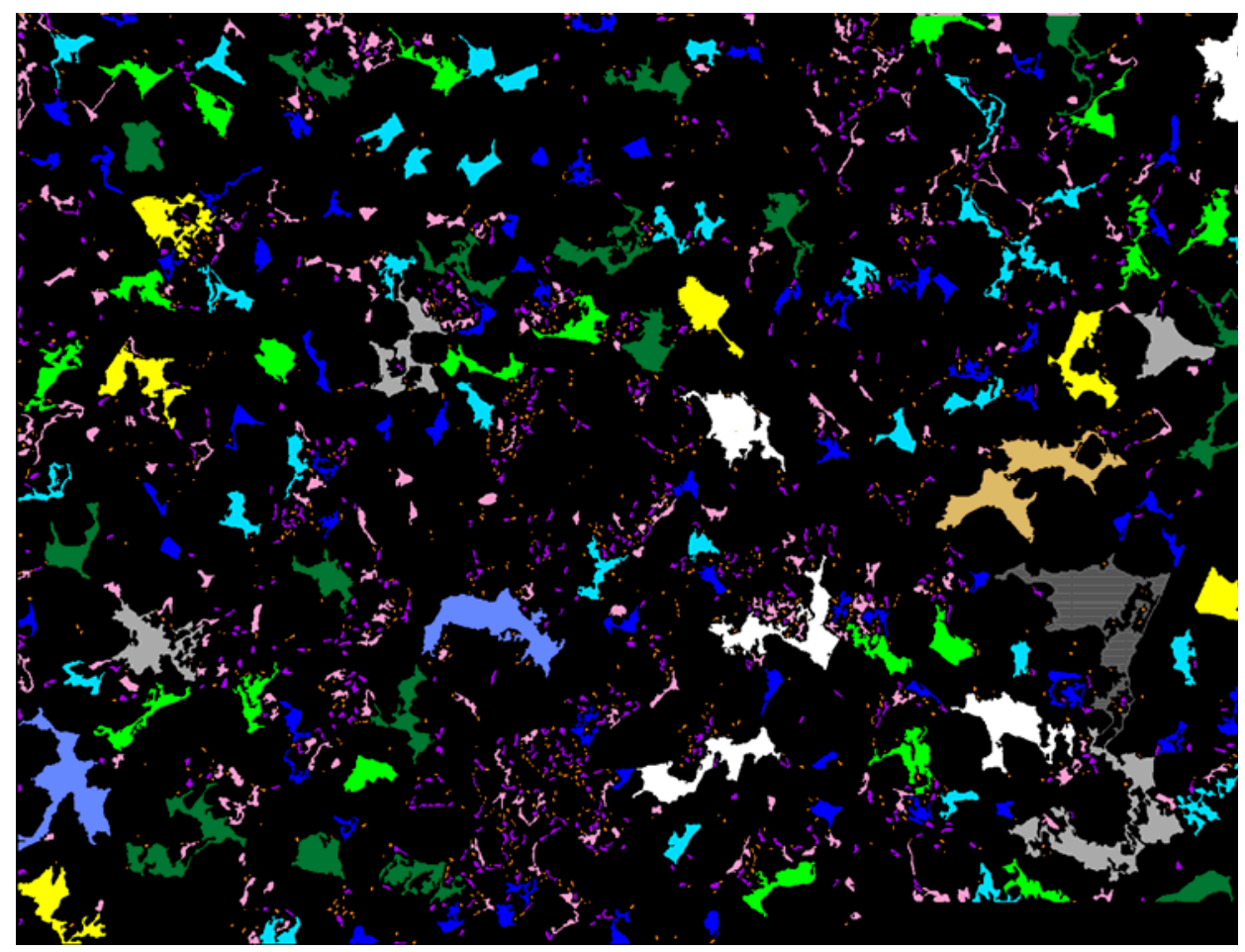

DOE

\section{Gas Hydrates Research}

Synthetic Porous Media

Sample Number:

Berea Sandstone

Depth (ft):

Porosity, percent:

Gas Permeability, md:

Grain Density $\left(\mathrm{g} / \mathrm{cm}^{3}\right)$ :

Sample Type:

Plug

\section{Scanning Electron Photomicrographs Description:}

\section{Plate 6A -}

Low magnification survey view of a fine grained $(0.22 \mathrm{~mm})$, well sorted sandstone. Framework grains are subangular to subround in shape and consist predominantly of monocrystalline quartz (G-8). Grains have rotated into a close packing arrangement in response to burial loading. Note development of long grain-to-grain contacts (J-13). Compaction is partially responsible for the loss of porosity and permeability experienced by this sandstone. Pore space is also partially filled by silica overgrowths (straight edges on white grains, C-12), dolomite and clay (L-6, C-6). 


\section{Plate 6B -}

High magnification view illustrating the partial occlusion of intergranular pore space by carbonate cement. Pore interiors are filled by booklet-like aggregates of authigenic kaolinite clay cement (G-10). Kaolinite particles are $<10 \mu \mathrm{m}$ in size and occur as booklet or vermicular masses that completely fill the interiors of scattered pores.

\section{Sample: Berea Sandstone}

\section{Plate $6 A$}

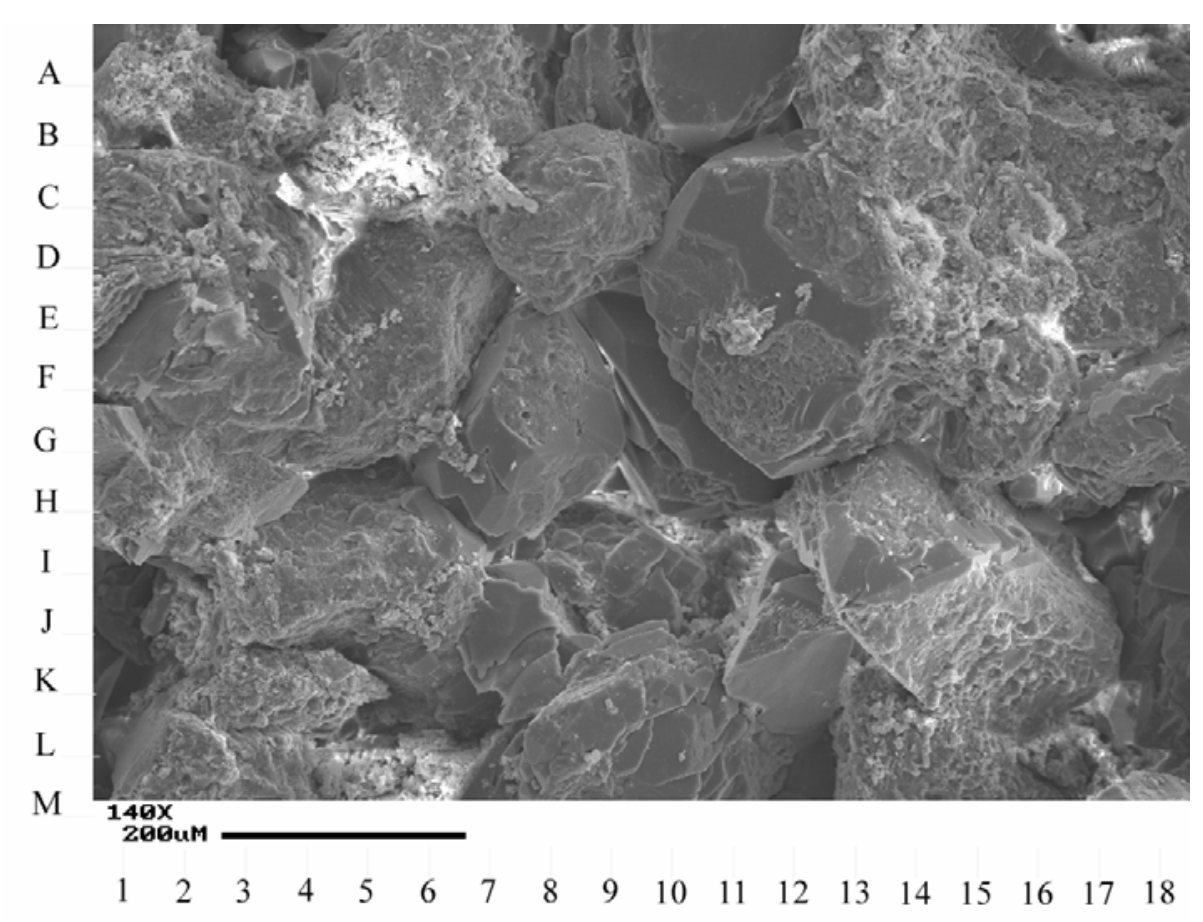

Plate $6 B$ 


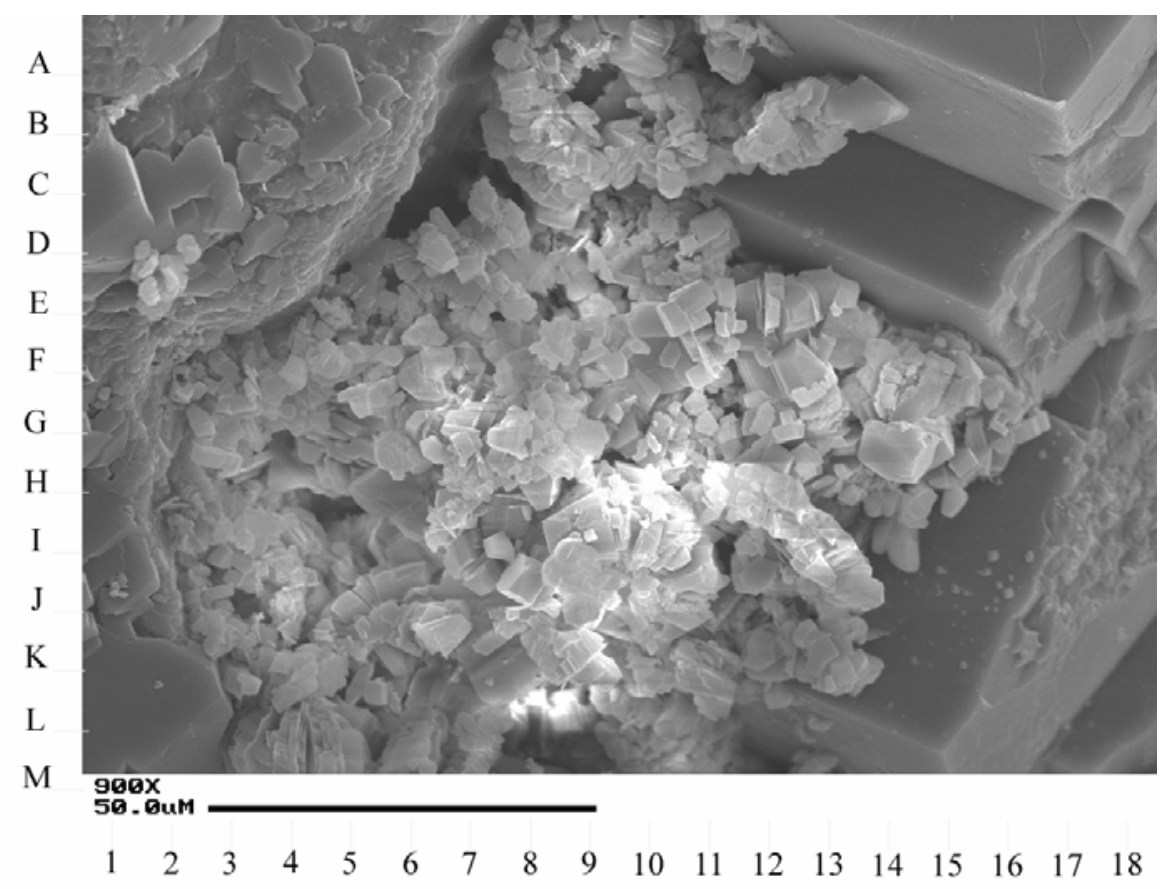




\section{APPENDIX B \\ User Manual for Hydrate Simulator Pre/Post Processing}




\section{INTRODUCTION}

This user manual describes the pre/post processor used in the modeling of laboratory experiments using the TOUGH-Fx/HYDRATE simulator developed at the Lawrence Berkeley National Laboratory. This simulator can model non-isothermal hydrate reaction, phase behavior, flow of fluids and heat in porous media. It has both equilibrium and kinetic options for hydrate formation and dissociation. The model can handle up to four components (methane, water, hydrate and an inhibitor) and four phases (gas, liquid, hydrate and ice). More details about this simulator can be found in the ToughFx/Hydrate manual supplied by Lawrence Berkeley National Laboratory.

In the depressurization step of the experiments, fluid was withdrawn at a constant volumetric flow rate. The simulator offers several boundary conditions, but constant total (including all fluid phases) volumetric flow rate at a well is not one of them. The pressure was well known at the production well during this depressurization and it varied with time. The simulator had an option of specifying a constant pressure boundary condition, but not a time-varying pressure boundary condition. Thus, the pressure decline was discretized into many (100) small steps as a function of time. The simulator was repeated for each step, taking the final state of the medium and inputing that as the initial condition for the next pressure decline step. This procedure needed certain amount of automation for this process. Also, the input and output format for this simulator was not user friendly. However, a process was developed to make it easier.

One of the major challenges in running the TOUGH-FX/HYDRATE simulator was the complexity of the input and output files. The input and output files were very similar to those of its predecessor, TOUGH2. The input and output files contained large amount of strings and required advanced string processing algorithms to extract the important data and disregard the unimportant ones.

An Excel/VBA code was developed as the Graphical User Interface (GUI) for the input file. The Visual Basic for Application (VBA) code uses string processing functions to 
extract information from the input file, and displays it in particular cells in Excel. Changes can be made to the various input values in appropriate cells and the VBA macro will use string processing function and make the input file readable by the TOUGH-FX/HYDRATE simulator.

In the case of the output file, Excel/VBA was not efficient to extract all the information. For this purpose a combination of a standalone $\mathrm{C}++$ code (TFXRead) and Matlab code (TFXPlot) was used to extract and visualize the data generated by the simulator. A C++ code was developed using GNU C++ in windows using advanced string processing. The code scans through the data files line by line and tries to match certain key words. If the keyword is found then the code splits the line into appropriate data sets and stores them in a separate text file. Results for each iteration are stored in a separate text file and numbered consecutively. To plot the data generated from the simulator, use MATLAB 7.2. This manual describes how to use the computer programs supplied in the accompanying $\mathrm{CD}$. 


\section{SYSTEM / HARDWARE REQUIREMENTS}

1. Pentium-4 of AMD Athlon XP with a minimum of $2 \mathrm{GHz}$ clock speed.

2. Minimum of $1 \mathrm{~GB}$ RAM. For larger systems $2 \mathrm{~GB}$ is recommended.

3. Minimum of $120 \mathrm{~GB}$ hard drive free space.

\section{SOFTWARE REQUIREMENTS}

1. Windows XP with all service packs and updates.

2. ToughFX version 1.0 (March 2005) - obtainable from Lawrence Berkeley National Laboratory.

3. Codeblocks with Mingw for $\mathrm{C}++$ editor as well as compiler. This is required only if you wish to make changes to the source code. Can be obtained freely from www.codeblocks.org.

4. MATLAB 7.2 (R2006a)

5. A good text editor. Some options are:

a. Ultraedit (www.ultraedit.com).

b. PSPad (www.pspad.com). 


\section{CONTENTS OF THE ACCOMPANYING CD}

The CD contains the following folders:

1. ToughFX - contains TFX.exe which is the ToughFX version 1.0 from Lawrence Berkeley National Laboratory. This executable performs the simulation.

2. TFXRead - contains TFXRead.exe which reads and processes the output files generated by ToughFX.

3. TFXPlot - contains a list of Matlab files $\left({ }^{*} \cdot \mathrm{m}\right)$

a. Pressure_stepping.m - used for producing pressure stepping code and further automation.

b. TFXPlot.m - Used for processing a single output file.

c. Analyze_data.m - used for processing, analyzing and plotting data from pressure stepping simulations.

4. Runs - contains the run files and this is where all further runs should be kept.

d. Test12_2 - contains the run for experiment no. Test 12 .

Windows XP and MATLAB are not provided in this CD. A separate license is required to be purchased.

The ToughFx manual is not included in the $C D$, but must be used to generate the input for the simulator. 


\section{INSTALLATION}

In any drive (C:, D:, ....) copy the folder 'TFXHydrate' and its contents which are ToughFX, TFXRead, TFPlot, Runs.

Try to avoid copying the folder and its contents in any other folder or changing the folder names. Otherwise, the paths need to be changed in the Matlab files. 


\section{CREATING THE SIMULATION INITIALIZING}

Before creating a pressure stepping, first create a sample input file with the right geometry, and all rock properties. For creating this input file, refer to the ToughFx user's manual.

The current pressure stepping code will work with the following options only:

1. R-Z coordinate system (Cylindrical coordinates).

2. Deliverability option for generation.

For illustration purposes, the current manual will use Test 12 from the experimental program (included in Runs folder) as an example.

Before creating any pressure stepping file, first take a look at the pressure vs. time ( $P$ vs t) curve. One such example is shown below. 


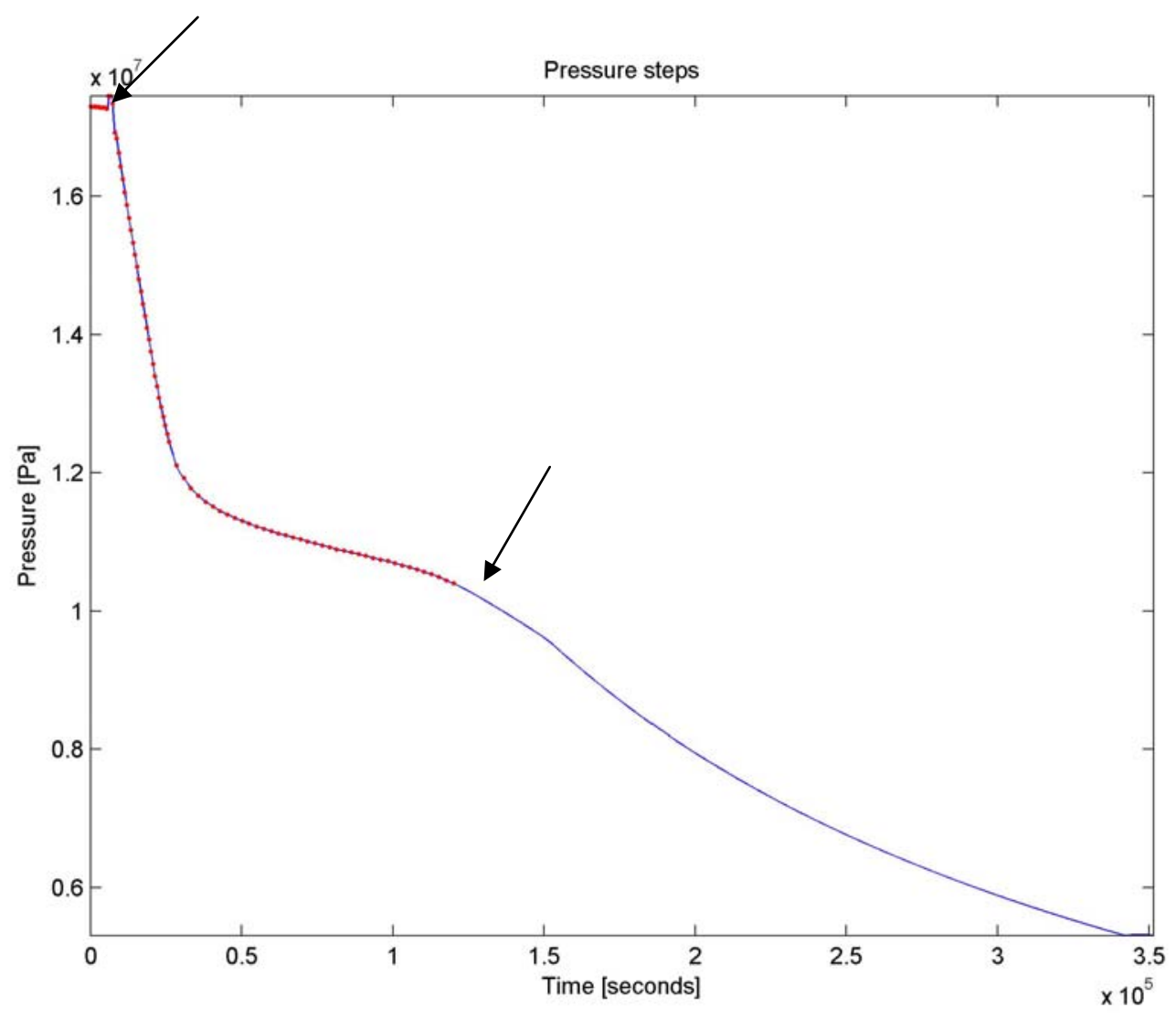

Figure 1: Pressure Vs. Time Obtained from Experiments

In the Runs folder create a folder called Test12_2.

In folder Test12_2, create a text file called t_p_test12.txt or any file name which should contain 3 columns.

1) $1^{\text {st }}$ column - time in seconds

2) $2^{\text {nd }}$ column - pressure in $\mathrm{Pa}$

3) $3^{\text {rd }}$ column - temperature in ${ }^{\circ} \mathrm{C}$

The data should be in the form shown in Figure 2. 


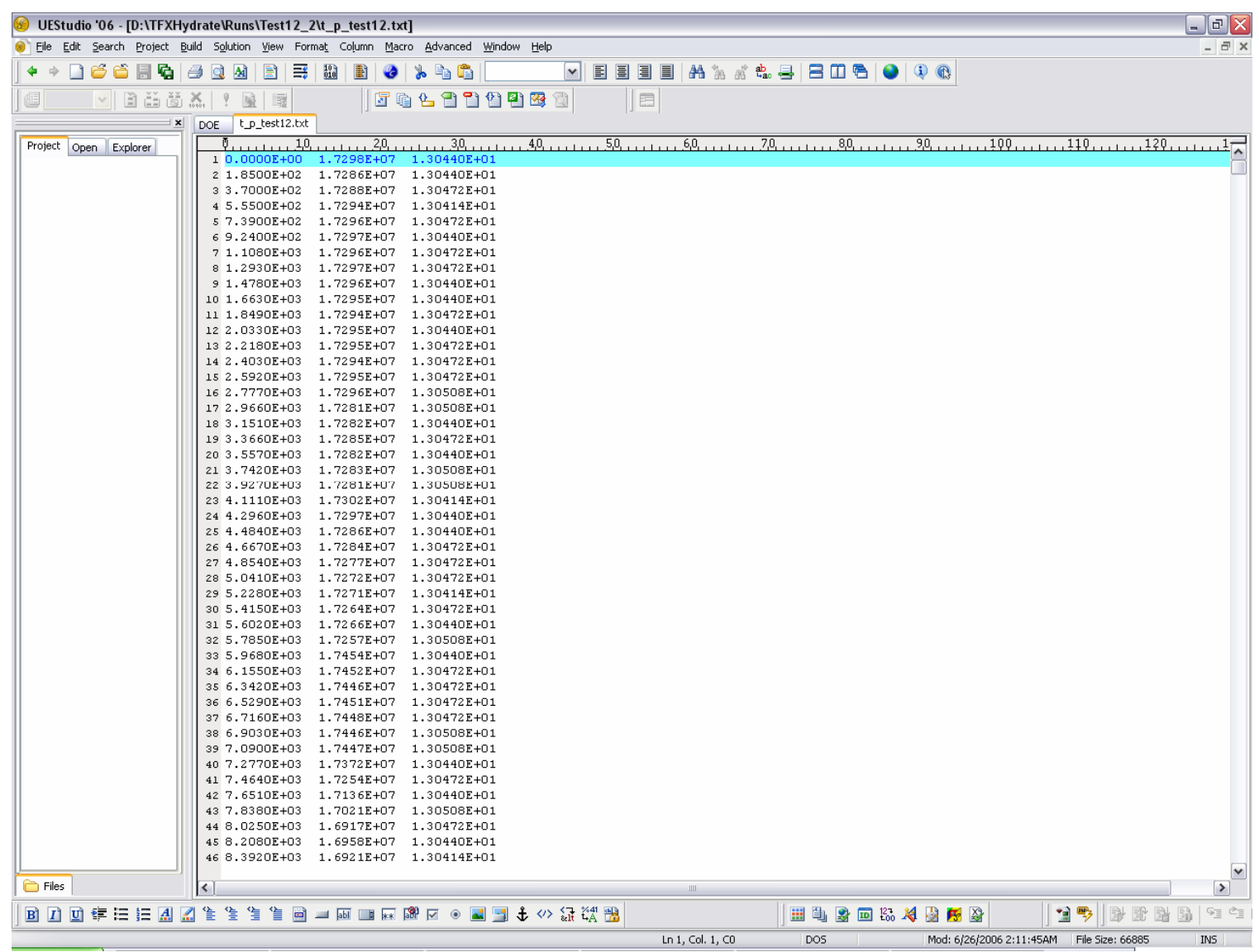

Figure 2: Data if Time, Pressure and Temperature

Create a sample input file called DOE with appropriate geometry, boundary conditions and properties in the folder. Use the ToughFx manual to create the file and put in folder Test12_2. The input file is shown in Figure 3. 


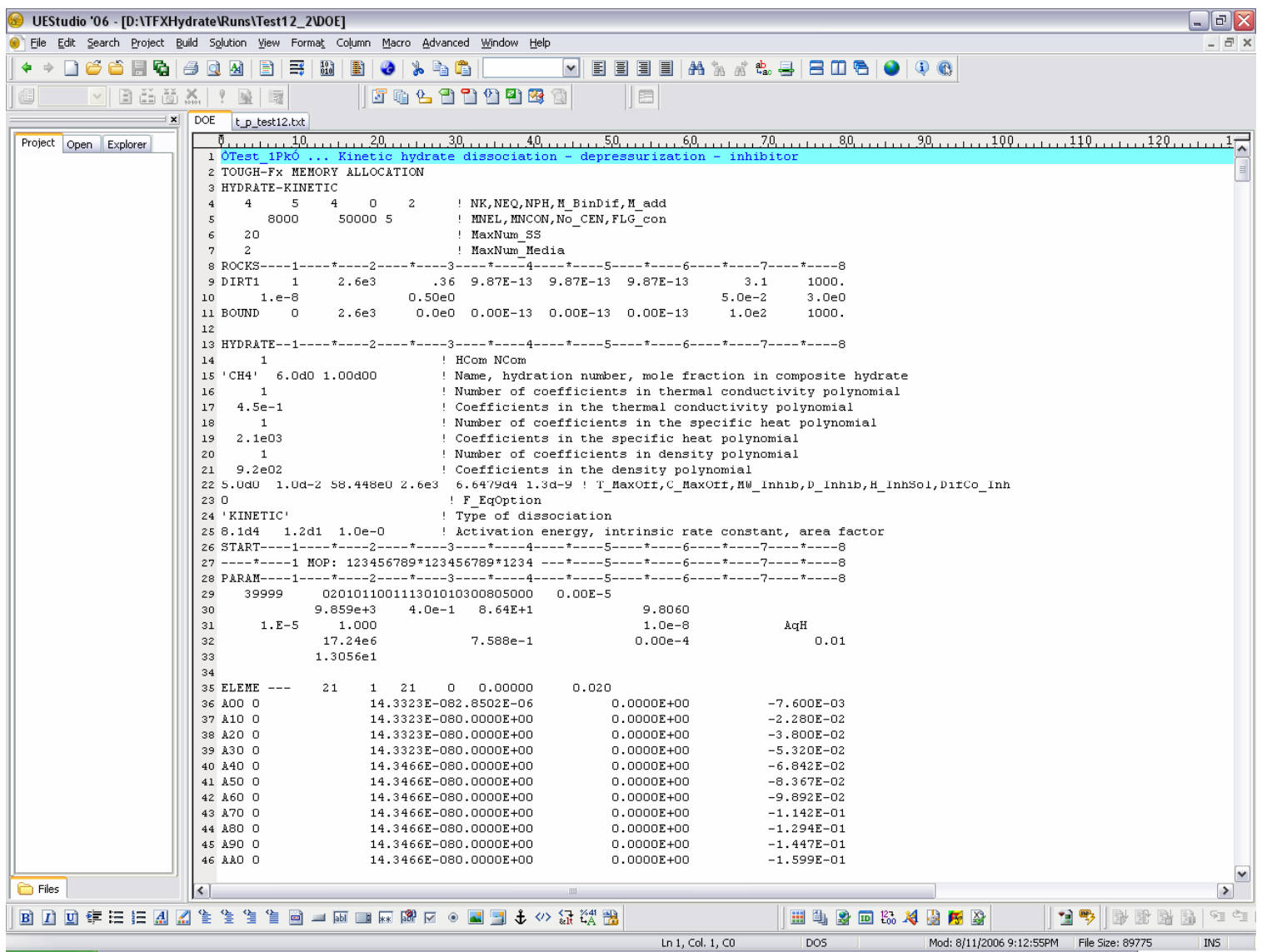

Figure 3: Screenshot of the Input File DOE 


\section{CREATING PRESSURE STEPPING INPUT FILES}

Now in Matlab open the file pressure_stepping.m. The file is shown in Figure 4. Now make the following changes in the file inside the Matlab editor:

\begin{tabular}{|c|c|c|}
\hline Line No. & Syntax & Comments \\
\hline 5 & is_see $=1$; & $\begin{array}{l}\text { To view the graphs and } \\
\text { steps }\end{array}$ \\
\hline 6 & runpath = 'D:/TFXHydrate/Runs/Test12_2/'; & $\begin{array}{l}\text { The path where the run } \\
\text { is stored }\end{array}$ \\
\hline 7 & tpfile = 't_P_test12.txt'; & $\begin{array}{l}\text { The data file with } 3 \\
\text { columns (time, } \mathrm{P}, \mathrm{T} \text { ) }\end{array}$ \\
\hline 8 & inpfile = 'DOE'; & The sample input file \\
\hline 9 & ngrid = 40; & $\begin{array}{l}\text { No. of pressure steps }< \\
100\end{array}$ \\
\hline 11 & grid_id = 'AJO'; & $\begin{array}{l}\text { The grids where well is } \\
\text { present }\end{array}$ \\
\hline 12 & grid_no = $0: 0$ & $\begin{array}{l}\text { The range if the well } \\
\text { grids in this case only } \\
\text { one grid }\end{array}$ \\
\hline 13 & well_id = 'WEL'; & The name of the well \\
\hline 14 & $\begin{array}{l}\text { GX = '1.64e-12'; } \quad \text { \% Productivity Index } \\
\mathrm{PI}(\mathrm{m} 3)\end{array}$ & $\begin{array}{l}\text { Change to appropriate } \\
\text { value (see TFX manual) }\end{array}$ \\
\hline 15 & $\begin{array}{l}\text { EX = '1.32E+07'; } \% \text { Bottomhole pressure } \\
\text { Pwb(Pa) }\end{array}$ & $\begin{array}{l}\text { Change to appropriate } \\
\text { value (see TFX manual) }\end{array}$ \\
\hline 16 & $\mathrm{HG}=$ '1.590E-2'; $\quad \%$ Thickness of layer $(\mathrm{m})$ & $\begin{array}{l}\text { Change to appropriate } \\
\text { value (see TFX manual) }\end{array}$ \\
\hline 18 & ixline_time = 30; & $\begin{array}{l}\text { Line where time is } \\
\text { present in the input file }\end{array}$ \\
\hline 19 & ixline_gener0 = 1261; \% GENER----1- & $\begin{array}{l}\text { Line where GENER - is } \\
\text { present in input file }\end{array}$ \\
\hline 20 & $\begin{array}{l}\text { ixline_gener1 = 1263; \% empty line after all } \\
\text { wells }\end{array}$ & $\begin{array}{l}\text { One line after where } \\
\text { GENER list ends i.e. } \\
\text { after the well list }\end{array}$ \\
\hline 21 & ixline_incon = 1268; \% INCON----1----*----2-- & $\begin{array}{l}\text { Line where INCON is } \\
\text { present in the input file }\end{array}$ \\
\hline 30 & $\operatorname{tmax}=12 \mathrm{e} 4$ & $\begin{array}{l}\text { The maximum time for } \\
\text { the simulation to run. } \\
\text { See from graph }\end{array}$ \\
\hline 32 & t_step1 = 2.6e4; & $\begin{array}{l}\text { This is the time if } P \text { falls } \\
\text { too fast and wish to } \\
\text { have more points. } \\
\text { Always }<\text { tmax }\end{array}$ \\
\hline
\end{tabular}


Now run the Matlab code and you will see a figure similar to Figure 1. If the steps look satisfactory, then go to line 5 and change the value from 1 to 0 and run again. Now Matlab will create a number of folders as shown in Figure 5. Matlab will also create other files. 


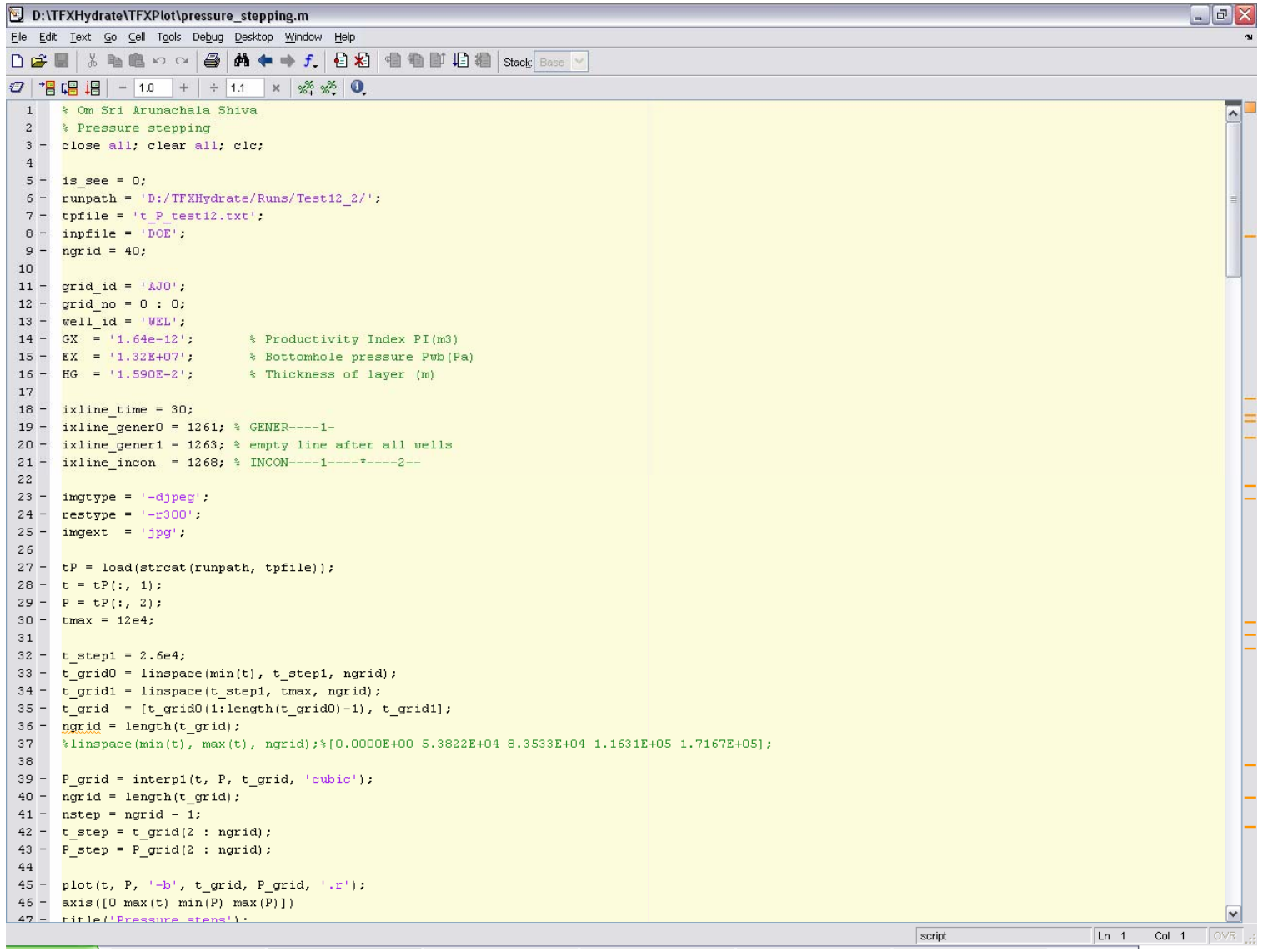

Figure 4: Screenshot of pressure_stepping.m 


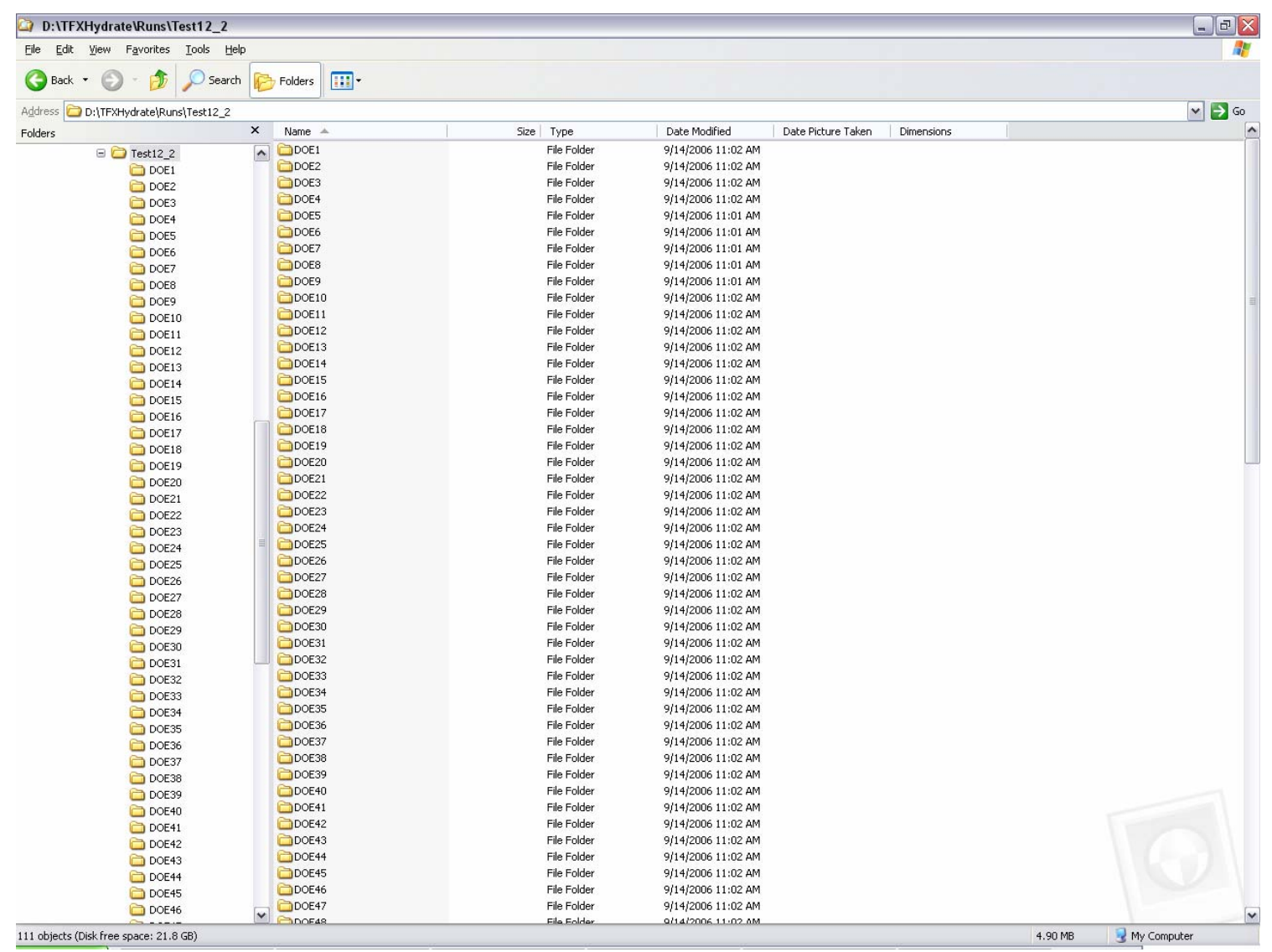

Figure 5: List of Folders Generated by Matlab 


\section{RUNNING THE SIMULATION}

Now open the command windows and type the following:

$$
\text { Run.bat }>\text { log.txt }
$$

And now the simulation is running. You can ignore messages such as:

'Could not find D:I TFXHydratelRunsITest12_2IDOE1l'

Wait till the simulation is complete. It will approximately take $30-120$ minutes to complete simulations depending the number of pressure steps and the computer.

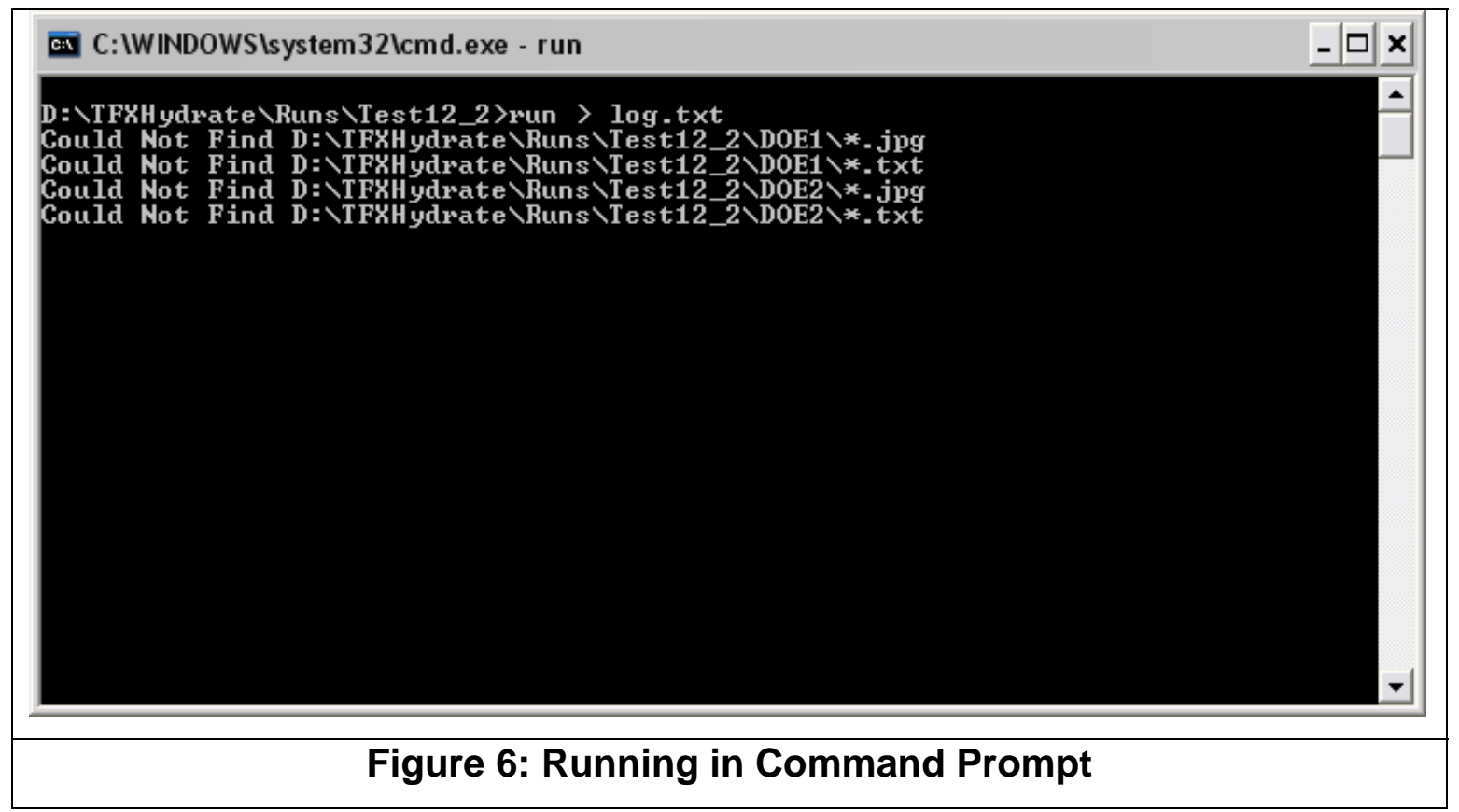

During the running, the batch file (run.bat) invokes the simulator to run the simulation on each of the input file and then calls the TFXRead to process the data generated by the simulator into readable format. All this is done automatically and hence user input is not required at this stage. 
Processing the pressure stepping input files:

After completion of the simulation, open the file analyze_data.m. This file is used for processing and plotting the data. A screenshot of the file is shown in Figure 7.

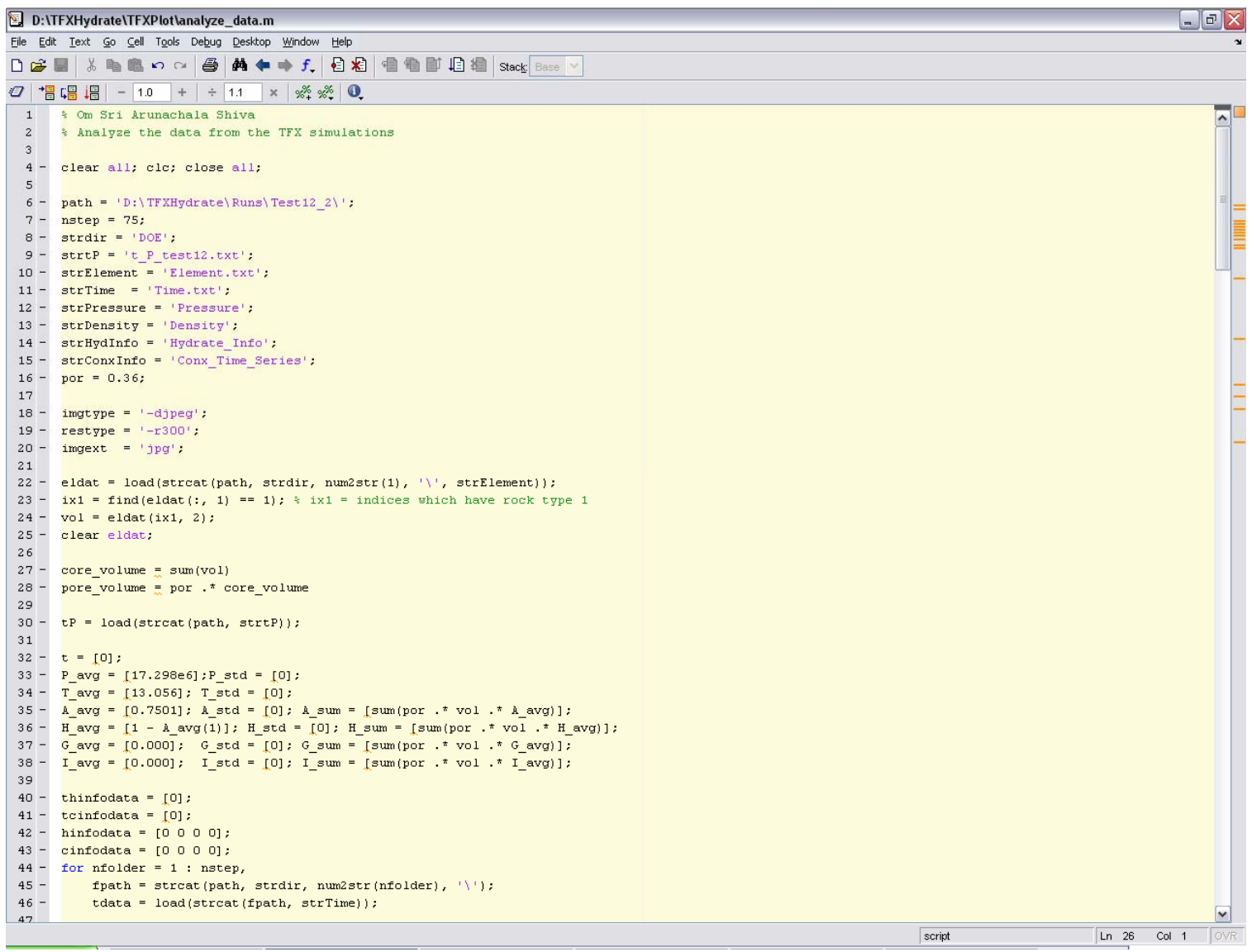

Figure 7: Screenshot of the File analyze_data.m 
Now make the following changes in the file:

\begin{tabular}{|c|c|c|}
\hline Line No. & Syntax & Comments \\
\hline 6 & path = 'D:ITFXHydratelRunsITest12_2I'; & $\begin{array}{l}\text { The main path where } \\
\text { the run is stored }\end{array}$ \\
\hline 7 & nstep = 75; & $\begin{array}{l}\text { Number of steps of } \\
\text { successful run. See } \\
\text { log.txt for successful } \\
\text { runs. }\end{array}$ \\
\hline 8 & strdir = 'DOE'; & The sample input file \\
\hline 9 & strtP = 't_P_test12.txt'; & $\begin{array}{l}\text { The data file with } 3 \\
\text { columns (time, } P, T \text { ) }\end{array}$ \\
\hline 16 & por $=0.36$ & The porosity of the core. \\
\hline 33 & P_avg $=[17.298 e 6] ; P \_s t d=[0] ;$ & $\begin{array}{l}\text { Change P_avg = initial } \\
\text { Pressure. Don't change } \\
\text { P std }\end{array}$ \\
\hline 34 & T_avg $=[13.056] ;$ T_std $=[0] ;$ & $\begin{array}{l}\text { Change T_avg = initial } \\
\text { temperature. Don't } \\
\text { change T_std }\end{array}$ \\
\hline 35 & $\begin{array}{l}\text { A_avg }=[0.7501] ; \text { A_std }=[0] ; \text { A_sum }= \\
{[\text { sum(por .*vol .*A_avg)]; }}\end{array}$ & $\begin{array}{l}\text { Change A_avg = initial } \\
\text { aqueous saturation. } \\
\text { Don't change other } \\
\text { values. }\end{array}$ \\
\hline
\end{tabular}

Now run the Matlab code. Matlab will process the files and create plots and text files. The tabulated values are stored as text files which can be used in Excel and the plots are stored as jpegs. All files are stored in the main path.

The text files generated are:

1. Conx_Info.txt - contains the connection data summed over all pressure steps.

2. Data_avg.txt - contains average values of $P, T$ etc over all grid blocks for all steps.

3. Data_sum.txt - contains saturation of all phases.

4. Data_vol.txt - contains total volume of all phases.

5. Hydrate_Info.txt - contains hydrate production data.

6. Pressure_steps.txt - pressure steps used for the simulation. 
The plot files generated are:

1. $\mathrm{CCH} 4$ _Mass.jpg - cumulative mass of $\mathrm{CH} 4$ produced.

2. $\mathrm{CCH} 4$ _MRate.jpg - mass flow rate of $\mathrm{CH} 4$ produced.

3. $\mathrm{CCH} 4$-Vol.jpg - cumulative volume of $\mathrm{CH} 4$ produced.

4. $\mathrm{CCH} 4$ VolRate.jpg - cumulative volumetric flow rate of $\mathrm{CH} 4$ produced.

5. CCH4_Vol_Conx.jpg - cumulative volume flow of $\mathrm{CH} 4$ between connections.

6. $\mathrm{CH} 2 \mathrm{O} \_$Mass_Conx.jpg - cumulative mass flow of water between connections.

7. Phase.jpg - saturation of each phase.

8. Pressure.jpg - pressure change.

9. Pressure_steps.jpg - pressure steps.

10.S_Aqu.jpg - saturation of aqueous phase.

11.S_Gas.jpg- saturation of gas phase.

12.S_Hyd.jpg - saturation of hydrate phase.

13. S_Ice.jpg - saturation of ice phase.

14. Temperature.jpg - temperature change.

15.V_all.jpg - all volumes of phases.

16.V_Aqu.jpg - volume of aqueous phase.

17.V_Gas.jpg - volume of gas phase.

18.V_Hyd.jpg - volume of hydrate phase.

19.V_Ice.jpg - volume of ice phase. 
